\title{
It's All One Curriculum: Activities for a Unified Approach to Sexuality, Gender, HIV, and Human Rights Education [Chinese]
}

International Sexuality and HIV Curriculum Working Group

Nicole Haberland

Population Council

Deborah Rogow

Follow this and additional works at: https://knowledgecommons.popcouncil.org/departments_sbsr-pgy

Part of the Demography, Population, and Ecology Commons, Family, Life Course, and Society Commons, Gender and Sexuality Commons, International Public Health Commons, and the Medicine and Health Commons How does access to this work benefit you? Let us know!

\section{Recommended Citation}

International Sexuality and HIV Curriculum Working Group. 2011. "It's All One Curriculum: Activities for a Unified Approach to Sexuality, Gender, HIV, and Human Rights Education [Arabic]," edited by Nicole Haberland and Deborah Rogow. New York: Population Council. 

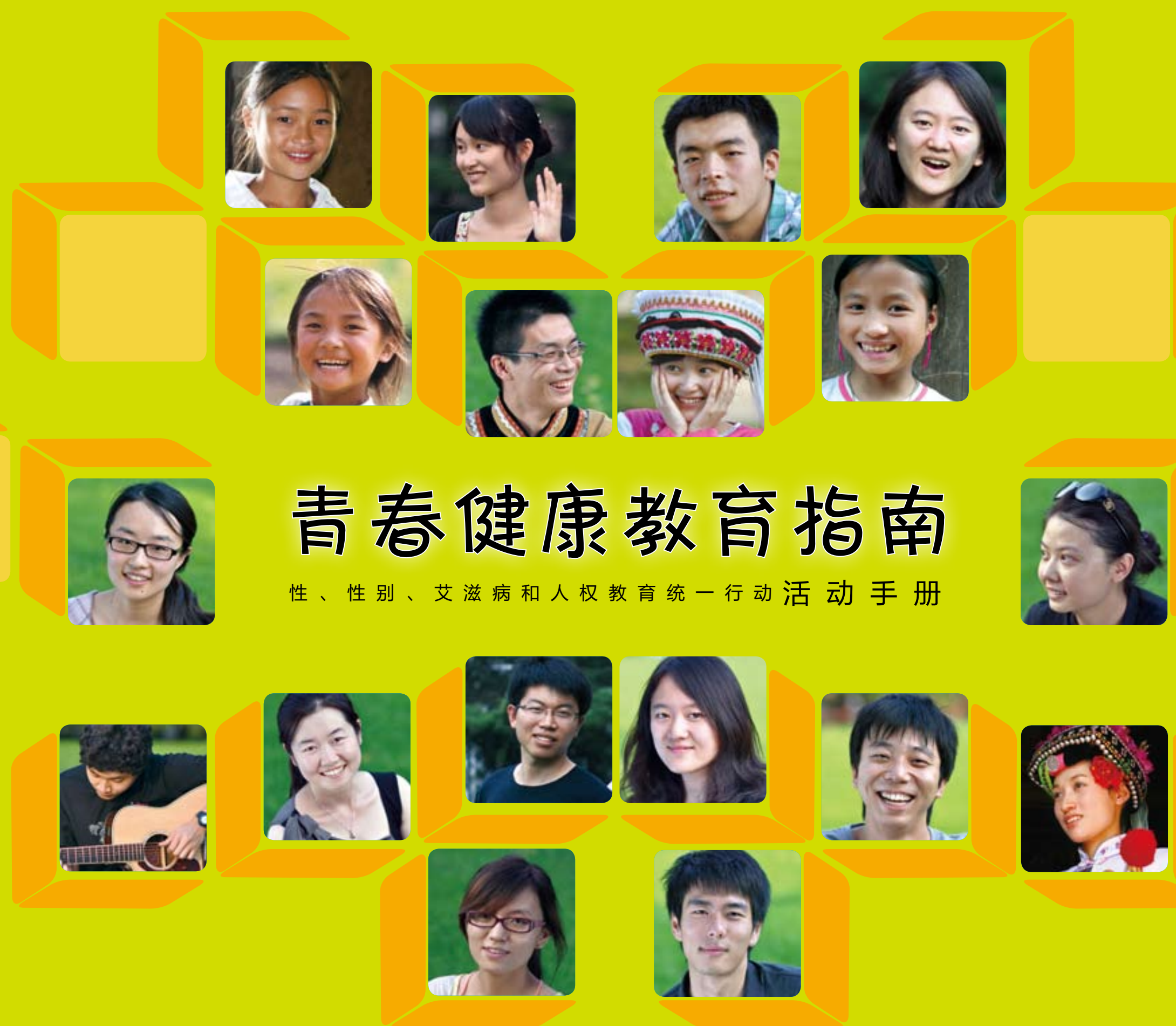
一国计划生育协会

中国计划生育协会是中国在计划生育/生殖健康领域最大的全国性群众团体, 成立于1980年, 1983年正式加入国际计划 生育联合会, 2005年获得联合国经社理事会非政府组织咨商地位。

多年来, 中国计生协积极配合各级政府, 团结广大育龄群众, 发挥志愿者的作用, 致力于计划生育、优生育, 促进人民 的生殖健康, 充分保护人们享有平等的生殖权利。在全国各地积极开展了形式多样、丰富多彩的项目动, 在预防艾滋病、青 少年性与生殖健康教育、维护妇女权益、发动宗教领袖开展生殖健康教育等众多领域显示出自己独特的活力和优势, 形成了 具有广泛影响的村居民自治、生育关怀、青春健康等一系列品牌活动。

地址: 北京市朝阳区䓁药居35号楼

邮编: 100029

电话: 86-10-8465-7984

传真: 86-10-8465-7975

电子邮件: cfpacfpa@chinafpa.org.cn

如需更多信息，请访问：www.chinafpa.org.cn

uww.chinayouthnet.org

《青春健康教育指南》是中国计划生育协会组织并协调翻译的出版物。欢迎大家使用本手册用于非商业性（非赢利性） 的项目活动和培训。但在使用时, 请注意下列问题:

若您根据当地的实际, 对本书的部分内容进行复制或进行适当的改编用于非商业性的项目活动或培训时,

请注明您所引用材料的出处。

若您复制、印刷全书，请事先与中国计生协办公室联系。

如果在您制作的培训材料中引用或改编了《青春健康教育指南》的内容, 中国计生协非常希望能够得到一份您制作的培 训材料。 


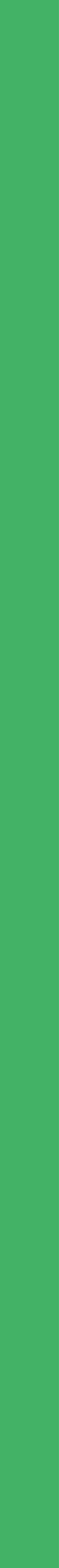


- 同 “教什么”一样重要的是 “怎么教”。在性、艾滋病、性别和权力的教育中, 最有效的方式是互动式的、以培 训对象为中心的参与式教学方式。事实上, 参与式教学方法可以帮助年轻人为未来他们更健康、幸福地生活在这 个错综复杂、千百万化的世界做好准备。

- 为了能够更有技巧和更便捷地运用参与式方法, 教育者们需要充分的准备并得到和相应的支持。本手册介绍了以 培训对象为中心的、互动式的参与式教学方法, 并提供了将参与式教学方法应用到性、艾滋病等课程当中的技 巧。本手册最后提供了如何在学校、社区等不同人群中开展培训的指南。

- 《青春健康健康指南》包括两本手册。本手册为“活动手册”, 包含54个活动案例。配套教材 ( 指导手册) 也支持互 动式的、以培训对象为中心的教育方式。例如, 指导手册中的穿插 “思考要点”, 鼓励学习者就某一话题进行批

判性思考; 第八单元还让学生在实践操作中学习。 
本手册使用提示

互动式的、以培训对象为中心教学方法 07

参与式培训的原则 08

参与式方法使教学更为丰富多彩 12

开发和使用本书需要特别注意的地方 政策性因素的考虑 13

计划性因素的考虑 14

资源严重掼乏时的课程开发 14

课程与学术标准相连 15

针对特殊人群的课程改编 15

教师培训前的准备 15

评估 16

寻求帮助并保持活力 16

\section{(1) 第一单元 性健康需要人权}

活动 1 为何公平很重要 22

活动2 关于 “人权” 24

活动 3 权力、特权、平等 29

活动4 关于性与生殖健康权利案例研究 31

\section{(2) 第二单元 社会性别} 活动5 男性和女性的词汇网络 38

活动6 记忆旅程: 孩提时期对性别的认识 41 活动7 父亲的形象 43

活动8 研究项目: 学校环境中的性别 45

活动 9 推销固有、刻板印象的观念: 广告分析 52 活动10 性别化的空间 54

活动11 女性活动家: 创作一本属于自己的书 57 活动12 对外表的改变 61

活动13 亲密关系: 从暴力到尊重 63

活动 14 反对性暴力 65

活动15 性别角色和规范的变化 67

活动16 说真话的力量 69 
3) 第三单元 性

活动 17 关于性教育的辩论 74

活动18 第一印象 77

活动19 关于欲望的真相 81

活动20 感受不同 85

活动 21 被同性的人吸引: 个人故事 87

活动22 性行为一一谬误和事实 91

活动23为什么我们做（或者为什么不做）：

有关性决定的强迫选择练习 96

活动24 界限在哪? 性选择和性强迫之间的连接线 99

活动25 关于同意 104

活动26 热点政策话题与个人回答 111

\section{(4) 第四单元 人际关系}

活动27 我的人际关系 116

活动28 他们两人之间发生了什么? 118

活动29 这是爱吗 123

活动30 决定是否发生性行为 125

活动31 在两性关系中我们如何共处 129

活动32 我在寻找什么 131

活动33 恋爱麻烦 134

活动34 我有说过么? 不同风格的交流方式 138

活动 35 积极倾听 143
5 第五单元交流和做决定的技能

活动36 练习积极的沟通 152

活动37 解决冲突的五个沟通步骤 155

活动38 谈论性与性健康 159

活动39 执行困难的决定 163

6 第六单元 身体、青春期和生殖 活动 40 你在我身上看到什么 170

活动 41 身体变化

活动42 青春期: 我们还在期待什么 175

活动 43 关于身体的有趣事实

活动 44 生殖: 不只是件私事

活动45 时时刻刻: 与怀孕相关的死亡事件 192

活动46 性传播疾病的事实 198

\section{(7) 第七单元 性健康}

活动 47 性别与安全套的使用 202

活动48 谈论性与安全措施 210

活动49 艾滋病只是一个名称 213

活动50 你想告诉我们什么? 你想了解什么? 217

活动51 避孕知识小游戏 220

活动52 站在她的立场: 停止妊娠的决定 227

活动53 受到鼓励! 做出改变! 232

活动54 我所关注的问题 236 


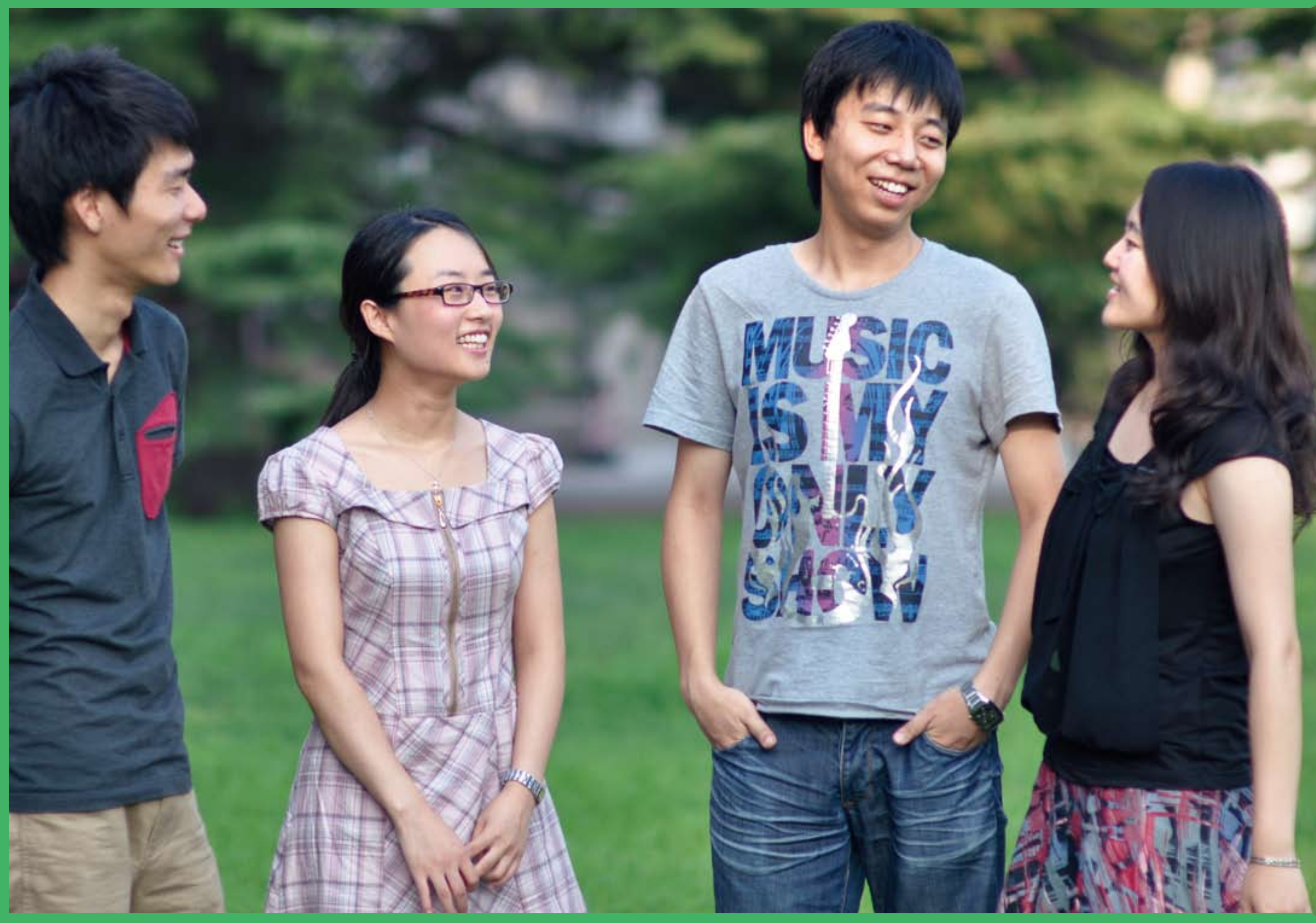


- 教育不是一个被动吸收知识的过程, 而是一个人们不断收集信息并在已有知识和经历的基础上重新 认知和理解的过程。为了让这一过程进行的更加顺利, 教育者应积极鼓励学生主动地寻求和参与到 学习中。

- 事实上, 很多专家已经证实互动式的、以培训对象为中心的教学方法相较于其他的教学方法来说, 教育效果会更好。这样的教学方式能够将学生纳入培训课程, 并促进他们批判性思考的能力, 特别 是对于那些会直接影响学习者生活的话题, 这样的方法能够帮助学习者更好地掌握知识并能够在实 际生活中运用所学的技能。

- 近几年来, 互动式教学方法已经成为性与艾滋病教育项目的最重要方式。越来越多的教育者运用这 种具有活力的教学方法, 并将 “亲身实践” 这样的教学形式带到各个领域, 包括社会学、公民学、 科学和文学。他们发现学习者在教学中的积极参与和自由的表达能够培养他们的民主态度。实际 上, 根据一个多国调查, 在开放式教学环境下学习的学习者更加支持性别平等, 教学者们也反映在 这种方式的教学过程中他们也学到了很多。

什么是互动式的、以培训对象为中心的教学方法?

此方法中的教学活动内容之间是相互关联的，能够让学生融入到教学活动中，并使他们积极地投入到学习中。 在下面几页将会详细介绍和阐释这个方法, 此方法主要强调: 学习环境、教学方法、批判思维能力、帮助学生 将能够将所学的技能应用到实际生活中。 
- 创建一个支持性的学习环境, 所有参与者都应该感觉到融入其中、被倾听, 并且感觉舒适和安全、 不被嘲笑的学习环境, 尤其是在他们发表新观点和想法的时候。鼓励参与, 尤其要鼓励那些感觉孤 独或者胆怯的人们 (不同的条件和情况下可能引起下面的感受。比如: 由于性别、社会阶层、年龄 等因素而导致人们社会权力的不同; 那些语言表达能力弱的学生可能会逃避讨论; 身体残疾或者有 其他特殊情况的学生可能会感到害差。）培训者可以通过促进相互尊重和团队建设的活动, 以及给 予积极参与者以分享和交流想法和经验的机会等方法让所有人参与到培训中。当然, 确保培训过程 的安全和私密性也很重要;

- 在培训开始的阶段, 让学生们共同订立关于本次培训的原则;

- 要求学生们尊重别人的隐私, 并提醒他们不要向他人泄露在课堂上大家分享的一些私密信息。考虑 到一些学生可能会利用培训中讨论到的敏感话题威胁他人, 向学生们承诺, 作为他们的老师, 将为 所有的讨论和分享严格保密, 这样您树立了一个尊重他人隐私的好榜样;

- 让学生明白, 如果教学中, 他们感觉不舒服的时候, 有权利选择不参加活动和分享观点;

- 鼓励所有学生分享他们的想法, 不要评判他们的观点。 
关于进行权利、性别、性、艾滋病、性健康等培训旧应特别注意的：

培训过程中, 学生可能会因为话题本身的敏感性或者自身及家庭的原因而感到不舒服, 培训者要注意避免不必要 的压力。

学生可能会与培训者分享关于意外怀孕、暴力经历或者其他的个人及家庭问题, 思考如何在保护学生隐私的基础 上帮助他们。了解当地的相关项目和服务, 比如, 为无家可归少年提供的安全的生活场所或者帮助被虐待妇女的 服务。涉及到毒品、药物等滥用或伤害的议题, 请向学生介绍国家在此方面的法律法规。

培训者需要考虑选取的培训资料是否适合培训对象。

本书支持基于平等、尊重和人权的价值观。培训者应反思自己的价值观，包括个人和宗教上的价值观，以确保你 能够接受本书的价值观。

2. 建立这样一个过程: 重视学生经验, 并把新旧知识融合在一起，思考相关问题

- 记住所有参与者都有自己的知识和经验。倾听, 或问一些问题以使他们把想说的话说出来:

- 了解学生们的关注点, 提出反映他们真实生活的假设性问题。鼓励学生通过交流和讨论解决这些问 题，做出自己的选择，并在这个过程中形成自己的想法;

- 鼓励学生探索, 假如一个事情发生在自己的生活中将意味着什么;

- 思考作为主持人, 你的角色是引发学生的思考, 而不是说教或者信息的灌输。有时你可能需要纠正 学生对一些事实错误的认识和理解, 或帮助他们认识到对他人不尊重的行为;

- 有时, 你可能希望与学生们分享自己的经验教训, 然而要注意与学生保持适当的距离。 


\section{3. 运用多种策略和技巧鼓励学生参与}

- 运用一些能够适应学生需求的教学方法;

- 如果你准备使用新的教学计划或活动, 首先请仔细审查活动内容和学习目标, 事先收集好相关的资料。

- 准备一份备用的教学方案, 以应对没人参与的滥於局面, 如时间、天气、学生不配合等原因;

- 可参考本书后附录列举的关于教学和课程的书目;

- 如有可能, 请尽量选择能让学习充满乐趣的活动!

\section{4. 建立学生对于自身和世界的反思、学习、批判性思考的能力及解决问题的能力}

- 鼓励学生对于传统思想提出质疑, 让他们思考他们的信仰和他们社会的标准和规范, 引导他们以尊重 的态度探索他人与自己不同的观点;

\section{本手册 使用提示} 参与式培训的原则

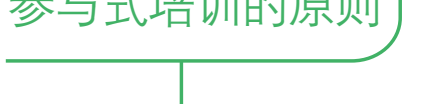

- 以开放式的问题展开讨论, 以 “苏格拉底对话法” , 通过问 “为什么” 引发学生对于矛盾的思考, 并 寻找更深层的真相。另一册书 (Guidelines) 的第一到第七单元都提供了 “思考要点” 鼓励学生进行 批判性分析和讨论;

- 鼓励创新;

- 鼓励学生们勇于思考, 不惧怕犯错误;

- 鼓励运用小组讨论和集体活动的方法;

- 分析问题时, 鼓励提出不同的观点, 并给出尽可能多的解决方法。 
关于进行权利、性别、性、艾滋病、性健康等培训时应特别注意的：

对一些敏感而特别的话题，可尝试先分男女进行讨论，然后再共同讨论和交流。如有必要，男女生可分别在不

同的房间里进行讨论。

5. 培养学生学以致用的能力，帮助他们成为积极的公民和积极变革的力量

- 选择有相关性的内容和带有批判性思想的教学方法, 反复要求学生将所学内容和他们自己的生活和世 界联系起来，鼓励他们本着公正和社会正义的原则展开思考;

- 将多种的学习方式有机的结合起来, 如社会服务、社会调查和实践活动。了解你工作地方的文化; 这 样教授的知识才不会让你的学生陷入困境;

- 考虑在你的机构或是社区中, 你是否并且怎样倡导年轻人表达并实践他们想法的能力和权利;

- 记住, 年轻人经常从他们的老师和社区领袖中得到启发和鼓励。

关于进行权利、性别、性、艾滋病、性健康等培训时应特别注意的：

注意学生可能会重新思考一些他们之前习以为常的基本假设。培训者注意帮助平衡, 学生们 在学着如何保 护他们家庭和社区稳定和安全时的理想和雄心。

你在教与性相关的内容时可能会遇到阻力，找到在你的组织和社区中，谁是你的同盟，谁有可能反对你，寻找 支持和指导。 


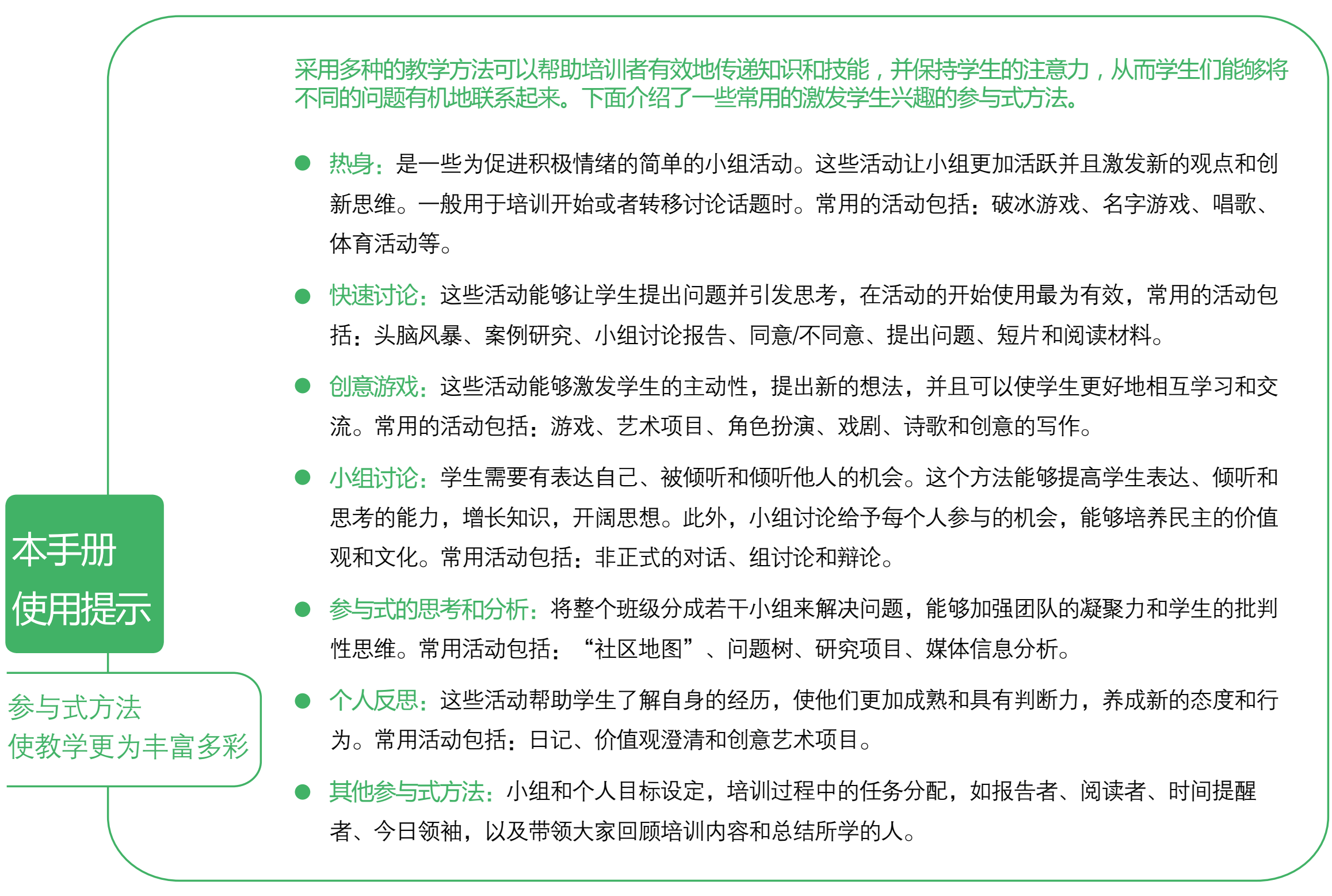


本书的编写基于世界通用的原则, 课程适合不同的培训对象。因此培训者可以通过提炼或者摘取 本书中的内容, 为当地培训对象开发一套行之有效的课程或者教学单元。这样做需要注意当地相关的 教育和工作状况, 其中包括教师的技能、学生的能力、家长的意愿、社会的价值观和课堂时间。下面 的问题是希望提醒您在使用本书时有需要注意的地方。有些问题可能是别人的责任一一比如, 执行机 构的工作人员和支持本项目的社区人员。强调这些问题是成功开展性教育、艾滋病传播预防培训的关 键因素，所以我们将这些问题归纳在此。（关于以上问题更多的指导信息请参考附加资源部分）

开发和使用 本书需要 特别注意的地方

有哪些全国性和地方性政策及资源影响性教育课程的开展? 比如, 有哪些性与艾滋病预防学校 教育的法律或者政策? 有何措施保证青少年能够获得性教育? 项目负责人是否注意到那些显示青少 年怀李、性病、性别平等以及人权之间存在着相互联系的研究? 设计项目时, 是否考虑到了这些关 联的因素? 你的项目或学校能否获得不同渠道的资助? 你的倡导目标是针对主要的决策者、还是你 所在组织的成员，或是其他个人? 谁是最重要的同盟? 


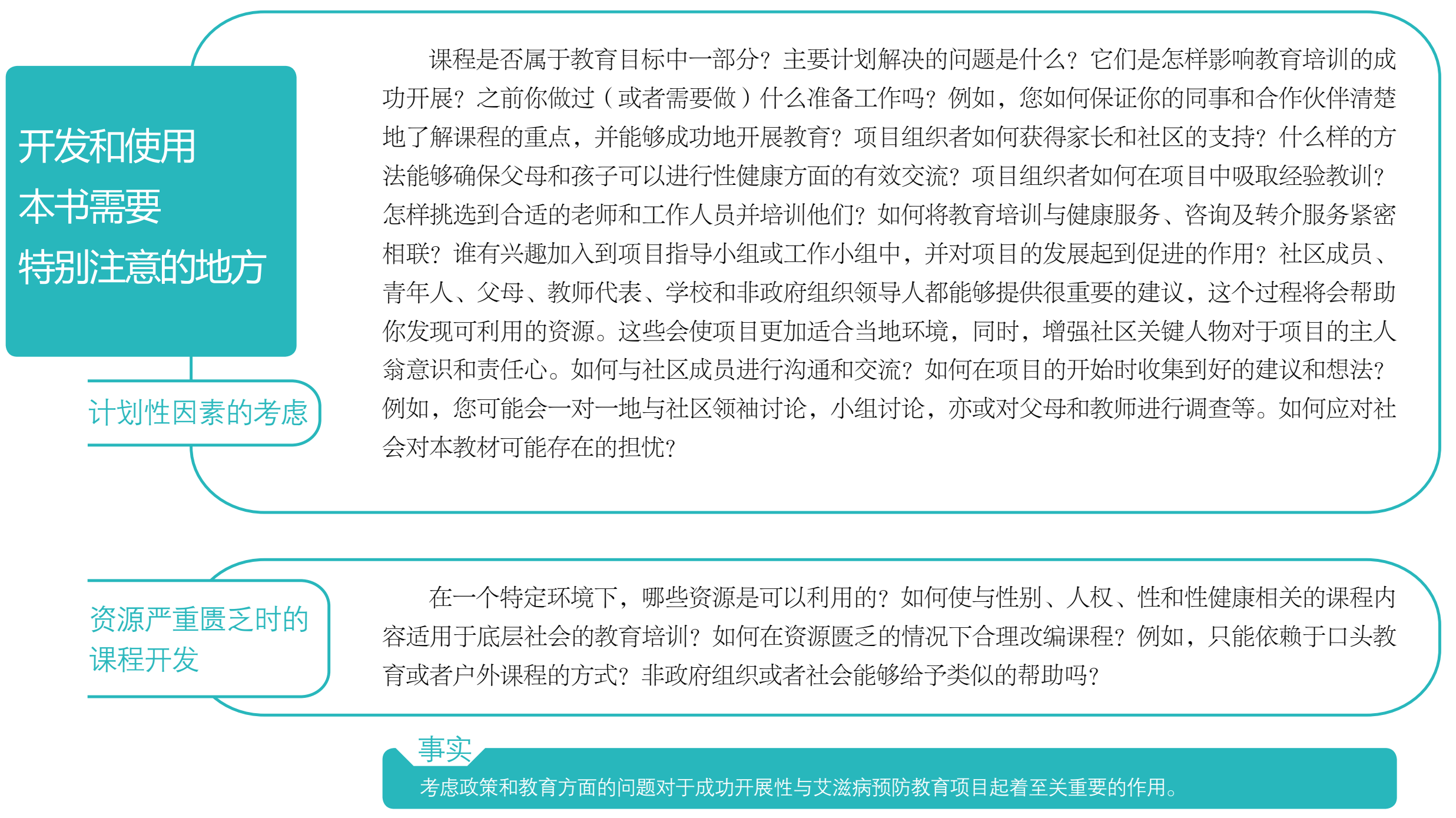


本书中的每个活动都包括一个与学术技能有关的目标 (例如, 说明文写作、叙述文写作、问题的 解决与分析、计划与实施一个简单的调查项目或者公众演讲）。这些内容除了可以促进将性与艾滋病 教育融入到学校教育中, 同时, 这个课程与教师和学校的教育目标相吻合。设计学校的课程时, 需要 考虑以下问题：需要了解和掌握哪些专业知识和技能? 这些专业知识和技能可在 “指导手册” 中找 到。哪些活动能够满足前面提到的目标? 结合不同的学科形成一个预防艾滋病、促进性别平等的方 法? 而不是与常规的课程发生冲突? 如何创新艾滋病教育以提高总体的教育质量?

开发和使用

本书需要

特别注意的地方

教师如何保证活动的内容能够满足特殊人群的需求? 例如, 如何为文化程度较低的人群开发适宜 的培训活动。是否能够保证培训内容反映了像青年农民工、已婚女性青年、士兵以及孤儿这样特殊人 群所关注的问题? 如何解决这些人在学习中所面临的困难? 能够提供一个安全的培训空间吗? 能够开 发时间较短的培训课程吗? 您如何找到那些与社区没有任何联系的学生? 哪些社区资源和转诊介绍是 学生们最需要的?

教师们是否做好了运用互动式、以学习者为中心教学方法的准备吗? 他们有机会提升这种技能 吗? 如何使用不同的培训方法将培训内容完美地传达给学生? 哪些培训能够使教师审视他们与性和性 别相关的自我意识和宗教信仰, 更好地传授符合人权的相关知识和技能? 如何保证教师在一个非常保 守的环境中有效地传递信息和技能? 


\section{开发和使用}

本书需要

特别注意的地方

很多教育者希望评估性与艾滋病教育项目的效果。哪些产出和标志能够证明我们完成了项目目 标? 例如, 学生们的知识、态度和行为是否改变? 项目是否会降低亲密关系当中的强迫和暴力的情 况发生? 是否使学生能够持续地学习? 是否有助于促进公民参与? 新的教育项目在试点阶段能够吸 取教训进行调整吗?

寻求帮助并保持活力

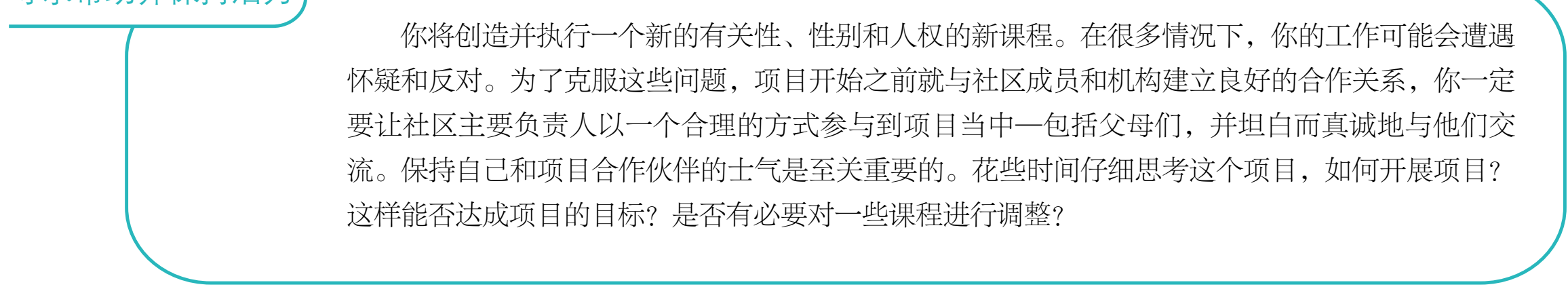


庆祝自己的成就异且尔要放弃努力! 


\section{欢迎使用《青春健康教育指南》活动部分}

这些活动是非常切实有效的

每一个活动的设计都考虑到整个学习的目标, 毕竟, 性与艾滋病教育可以并应该加 强总体学术的成果

为了达成总体目标，这些活动围绕两种类型学习目标紧密展开：1）亲身实践，2）加强特别的 技能，例如更缜密的批判性思维方法、写作技巧、调查技巧、公众演讲技巧、合作和解决问题的技 巧。更重要的是, 由于这些活动很有意义并且非常有趣, 可以使学生更好地融入到学校中, 并与学 校建立良好的关系。

每一个活动都与指导手册上的具体内容相对应

确实，一些活动使用到了指导手册中的事实说明表。

( 可以通过www.popcouncil.org/publications/books/2010_ItsAllOne.asp下载）

所有活动适合十五周岁及更年长的青少年朋友使用。一些活动也可用于十五周岁以 下的少年朋友使用

活动具体情况请参见下边罗列的适合于10-14周岁儿童的活动。

对于15周岁以下的学习者:

虽然本书理论上是为年级更大的青年朋友们编写的, 但是很多活动还是能够适用于或者改 编以后适用于十岁左右的儿童。

对于这些年纪更小的学习者，如下罗列了 21 个较有帮助的活动:

第一单元, 活动 1 第二单元, 活动 $5-6 、 8-11 、 15$ 第三单元, 活动 $18 、 20$ 第四单元, 活

动27、29、32 第五单元, 活动 36 第六单元, 活动 $40-43$ 第七单元, 活动 49-50 活动需要使用到学生阅读材料、工作表、答案提示和背景材料

通过这些便利的工具, 基本上随时可以开展培训活动。为了确保满足学习者的需求, 请阅读下 一页的注意事项。 
- 学校项目: 为了使培训更加有效, 大部分活动应该分别设计成一节课, 40分钟 (偶尔可能 是 2 到 3 节), 正如上一页所提到的, 这些活动应该包括学术目标, 因为这样会使培训更加 有意义和有趣, 能够加强学生与学校的联系。

- 社区项目: 接下来的活动更倾向于使用 “学生” 而非 “参与者” 我们选择这个词汇是因为 其简洁性, “学生” 并不只是狭义的指坐在教室里的学生, 无论我们是否在校园里, 我们 每个人都是学生。

- 为文化较低者设计的项目: 虽然很多活动当中都包括写作, 但是当培训群体文化水平较低 的时候可以将这些活动改编为口头的活动。比如: 可以利用图画卡片展开讨论。

- 让计划更加充分: 每个活动都包括概述、学习目标、活动时间、所需材料以及需要的准备 工作和步骤。培训之前至少阅读两遍培训资料, 能够帮助你知道大体的培训效果。在正式 培训之前, 可以找几位同事和朋友作为听众进行试讲, 以取得较好的培训效果。当然, 在 准备每节课的时候, 你需要让自己熟悉和此活动对应的指导手册中的相关内容。

- 为不同文化背景的群体设计的项目: 此手册的活动可适用于不同的文化背景环境中。然而 你可能会发现一些活动相较于其他活动更适用于你所在的环境。为了使活动更贴近学生并 且让他们感到更加舒服, 有时候你需要改动案例中的名字和和背景情况。

- 选择活动时需注意: 本书当中的活动组成不是一个单一的教程, 而是你在设计自己的培训 计划时可以选用的一些范例活动。你可以按需选取活动配合你培训的任何演讲、写作文 章、短片或者其他活动，在本书中的最后附有相关的补充材料。

- 准备材料时需注意: 很多活动要求准备粉笔和黑板, 当然您也可以使用白纸、马克笔、白 板等替代物品。

- 培训时间需注意: 活动的时间长短是非常灵活的, 取决于培训对象的多少、特点以及你为 讨论预留出来的时间。在活动的最后为讨论留出时间是非常重要的。 
性健康需要人权 


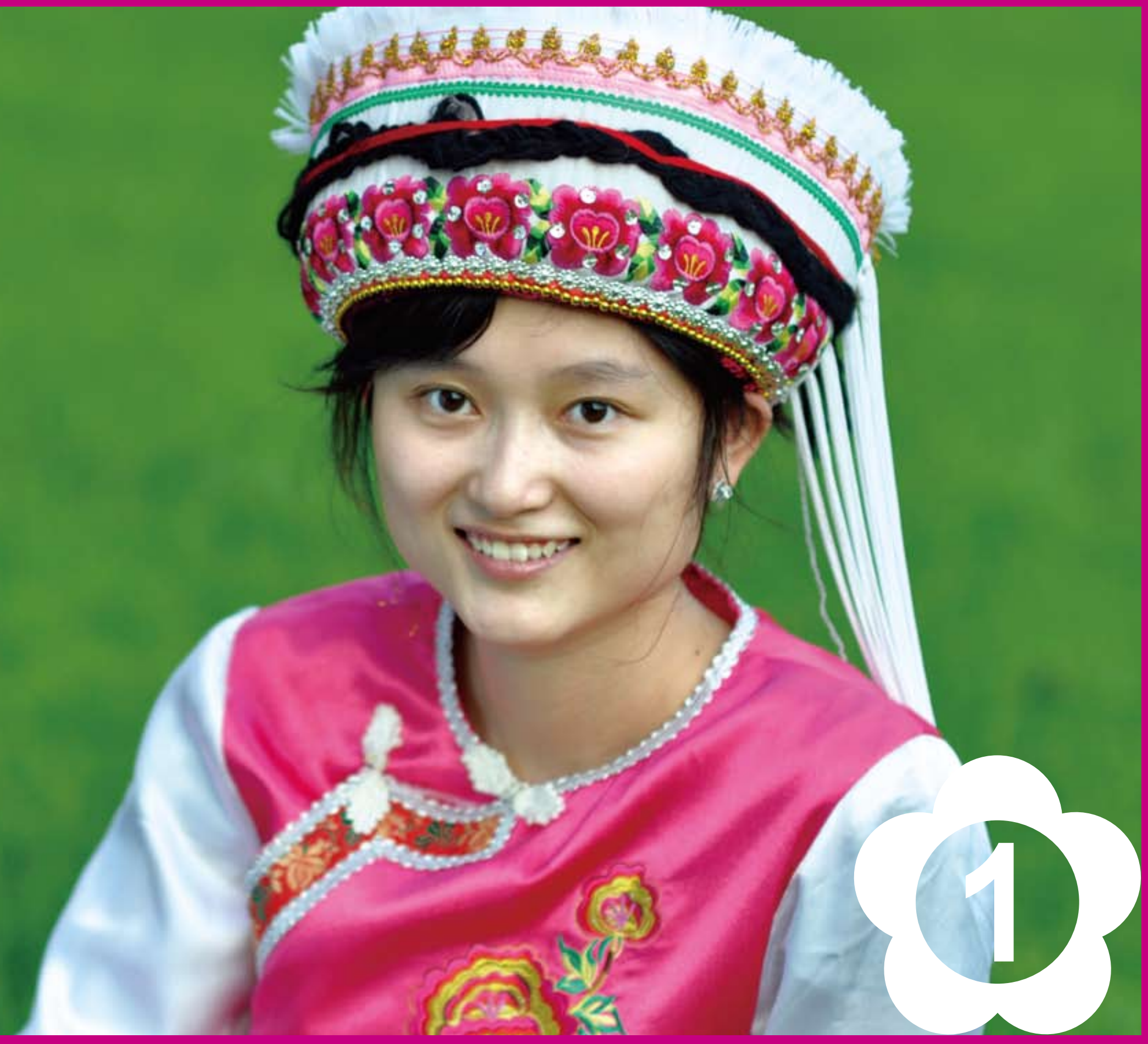




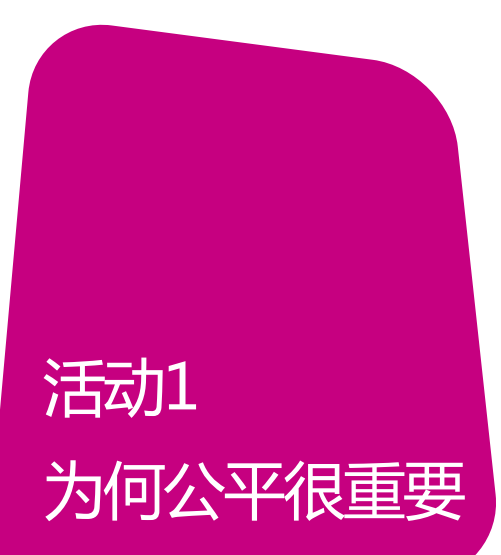

时间：步骤1-2：15分钟（可在第3步前一天当做作业布置下去）步骤3-7：40分钟

材料：黑板、粉笔

准备工作 : 提前预习引言, 复印所有引言发给每个学生, 或在课前把所有引言抄在写字 板上。决定 要不要在第3个步骤之前, 将第1-2步骤布置为作业, 让学生课前完成

概述：学生们通过讨论一些名人名言来思考公平这个问题

目的：帮助学生理解公平的重要性, 以及公平与人权之间的关系; 增强分析和写作技巧

1. 向学生们解释 : 这些引言都是由世界名人提出的。让学生们大声朗读这些引言，每次一个，暂不要 讨论。

- 公平就是正义的真谛;

- 妇女们正面临绝境, 并非为疾病所迫, 而是社会对她们的不公, 致使她们无法得到合理的救治;

- 我们只有对其他人的孩子以公平相待, 才能创建一个美好未来;

- 要善待他人, 因为你生平遇到的每个人, 都正在一场鹿战中苦苦拼搏;

- 公平待人, 直到所有人都能坚守公平;

- 公平的光芒让群星都熠熠生辉;

- 要好好活着, 这样当其他人想到公平和尊敬的时候, 他们就会想起你;

- 不管是在我们心中, 还是在法律上, 不论种族、性别、年龄和政治信念或宗教信仰, 我们都应以 公平和尊重待人。 
2. 向学生们布置以下任务:

- 选一个最能激励你的名言;

- 写下自己对于这句话的理解, 要求至少写一页纸, 解释一下为何它是 “对你说的” 或 “为何它能激 励你” ;

- 从你的生活中, 或从在你的社区中, 或从其他地方的事情中, 选取你认为上述名言可以有所帮助的 例子。这个例子可能是取自你被错待, 或你没有公平地对待他人, 又或者你目击到其他人之间有相 互影响的时候;

- 为何这句话传达的信息能产生作用?

- 之后你可以向同组其他人宣读你的作品, 当然这并非强求。

- 有人对这个任务有疑问吗?

3. 问你的学生有谁自愿分享他们写下的问题答案。（如果你已经收集和阅读了这些回答，你亦可从中 选一两个学生，请他们把自己的回答拿来与人分享。）之后，感谢这些与大家分享的学生。

4. 如果前两、三个学生都是在讲述他们自己受到不公平待遇的经历,那么询问一下，其他学生是否写到 了一些他人被不公平对待，或不公平对待他人的实例。

5. 感谢发言的各位学生，然后提出以下问题:

- 做到公平是否总是很容易?

- 请看引文的最后一段（“不管是在我们的心中...”）, 然后回答;

- 在我们的心灵深处,我们是否真正地公平和尊重地对待所有的人? 我们的法律公平、尊严地对待所有 人吗? 谁能举出一个例子? (如果可能的话, 请鼓励同学们从他们自己的社会生活环境中, 或者从 他们所听到的其他国家的事件中, 举出实例。) 


\section{教师参阅信息}

本活动的所有引文均来自 于以下个人 ( 按第一步中引文 出现的先后排序 ) : Justice Potter Stewart; 国际医学领导人 Mahmud Fathalla; 古希腊哲学家 Philo; 演员Alan Alda; 佚名; 作家 H. Jackson Brown, Jr.; 前美国总 统比尔・克林顿。

\section{6. 告诉所有学生 :}

- 人们并非一直以来就认同相互公平和尊重对待的含义, 也并不认同享受公平对待的权利。而只在 近50多年里，国际社会才开始一致认为,每个人都理应受到尊重并被有尊严的对待。

- 关于公平，人人享有一定的基本保护和权利。这些权利叫做 “人权” ，即指作为个人和社会成 员, 我们应怎样相互对待, 这只是因为我们都是人类。

7. 请学生进行讨论并回答：

- 你能想到一个人应享有的一项基本权利吗?（探讨这类权利如：食物、住宿、教育和安全。)

- 是否每个人都事实上享有这些基本权利?

- 重新回到你们所写的引文。引文中关于公平的说法适用于人权这个广义的范围吗?

时间：步骤1-2: 40分钟 步骤3-5: 45分钟

材料：黑板、粉笔，至少一份给培训教师用的《人权宣言》的副本

准备工作：这项活动应该是在学生们已经探索了人权所反映的价值这一基础上开展的, 比如说平等和 非歧视。查看《人权宣言》的内容, 并确保了解每一项权利的含义

概述：学生们设想一个新的国家的人民应该拥有哪些权利, 同时把这些权利和《人权宣言》做比 较, 并采访成年人对 “人权” 的理解

目的 : 使学生能描述人权的概念并能说出几项基本的人权, 增强他们抽象思维的能力 
1. 将学生分组，每组四或五个人。告诉学生们：

- 你们将被安置在一个岛上, 那里有一切生活必需品, 但是没有法律也没有历史。

- 你们必须拟订10条适用于岛上所有人的人权。

- 你们不知道任何人的地位、性别、民族、阶级和性取向。你们有10分钟来写这个列表。

2. 让每一组介绍他们写的列表。撰写一份统一的列表，其中包含所有组提到的人权。

3. 向学生介绍《人权宣言》，并告诉他们这上面的权利是世界上每个人应享有的。让学生们依次大声 读出《人权宣言》的每条条款，读完后，问学生对此有没有疑义。

4. 让学生们返回到他们的小组中，并给出10分钟思考下面问题：

- 找出我们总结出来的人权列表中与《人权宣言》里相似的点。

- 《人权宣言》中的哪些人权是我们没有列入的?

- 我们还想往我们的列表上面添一些人权吗?

- 在我们的列表上有《人权宣言》没有提到的条目吗?

5. 让所有的组回到讨论中来，并回顾他们对第4步中考虑的问题所做出的回答。问他们：有一个普遍同 意的、适用于每一个人的人权列表有多重要。

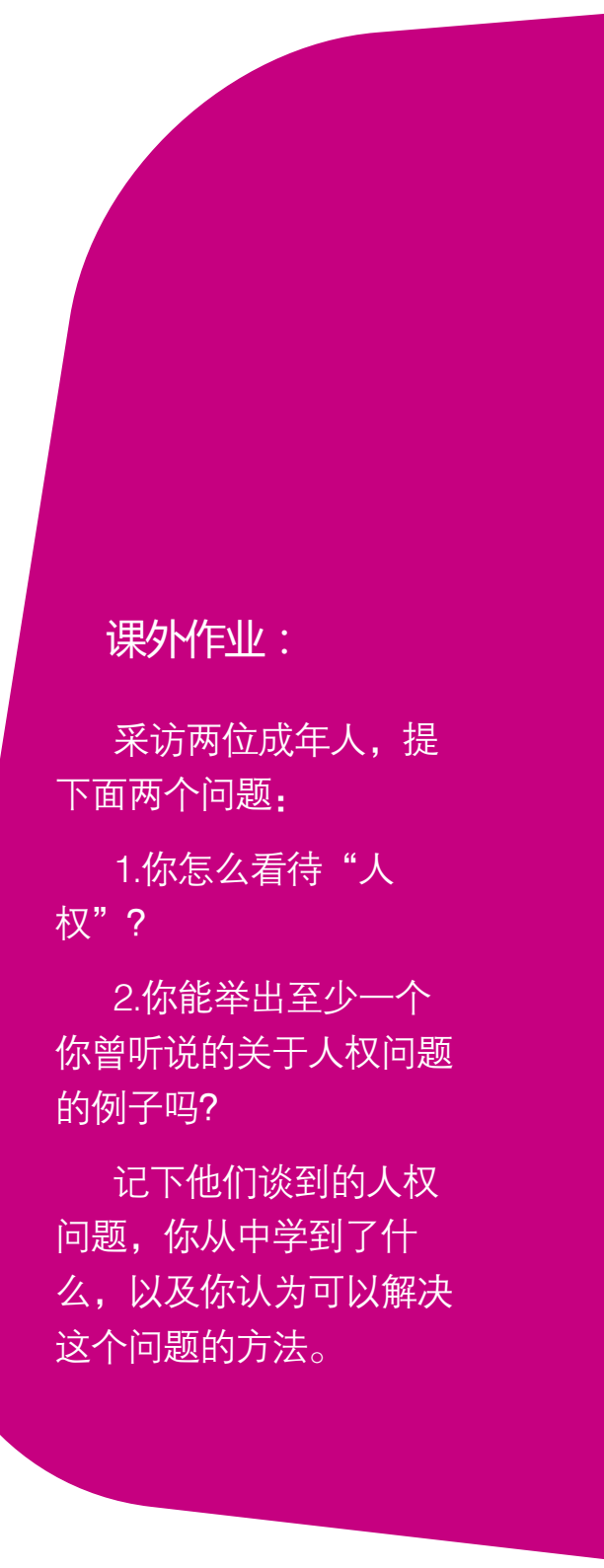




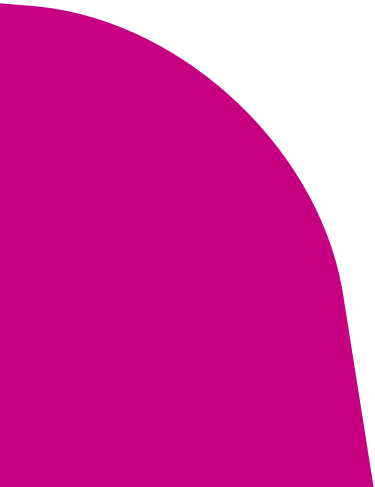

教师参阅信息

以下的列表用简单的语言 解释了每一项人权, 官方英 文宣言详见:

$<$ www.ohchr.org/EN/ UDHR/Pages/Language. aspx?LangID=eng $>$.
用简单语言解释《世界人权宣言》

第一条: 人人生而自由平等。人都是有理性有良心的, 并应该友好地对待他人。 第二条: 不论男女, 人人都拥有如下权利:

性别权;

- 肤色权 (种族权利) ;

- 言论自由权;

政治信仰自由权;

宗教自由权;

富贵或贫穷;

- 自由结社权利;

- 选择出生地的权利;

- 无论国家独立与否;

第三条：人人有权享有生命、自由和人身安全。

第四条：任何人无权把你当做奴隶来使唤, 同样你也无权让任何其他人成为你的奴隶。

第五条：任何人无权虐待你。

第六条：任何人在任何地方都应该受到同样合法的保护。

第七条: 法律面前人人平等。

第八条: 当国家赋予你的权利没有得到尊重时你有权获得法律帮助。 
第九条：任何人无权不公正地、任意地监禁你，或驱逐你离开自己的国家。

第十条：审讯必须公开举行。审判者不得受他人意见的影响。

第十一条：任何人在其未被证实有罪以前应被视为无罪。如果你被控告某一项罪名, 你有权为自己辩 护。任何人都无权对于一些你没做过的事情而定你的罪或惩罚你。

第十二条: 当你的名誉, 住宅, 通信, 家人无正当理由地遭到他人侵犯时, 你有权寻求法律的保护。

第十三条：你有权在本国自由行动，也有权离开本国前往另外一国并可自由回国。

第十四条: 如果你遭到他人伤害, 你有权去另一个国家寻求庇护。但是如果你严重违反了人权, 你也 会失去这项权利。

第十五条：你有权享有一个国家的国籍，他人无权以非正当理由阻止你加入另一国国籍。

第十六条: 一旦得到法律的允许, 男女就有权结婚和组建家庭。种族、国籍和宗教都不能成为阻止人 们行使这项权利的障碍。无论是已婚还是离异, 男女都享有同样的权利。任何人都不该强迫其他人结 婚。你所属国家的政府应该保护你和你的家人。

第十七条：人人有权拥有财产，任何人不能无正当理由地夺走属于你的东西。

第十八条: 你有宗教信仰自由权, 有权改变自己的信仰, 并有权自己一个人或是和他人一起实践宗教 仪式, 表达自己信仰。

第十九条：你有思想和言论的自由权，任何人无权禁止你这样做，而且你有和他国的人民分享你想法 的权利。

第二十条：你有组织和平集会、和平参加集会的权利。任何人不得强制你加入任何一个组织。 
第二十一条: 你和他人同样享有直接或通过自由选择与你看法相同的代表参与治理本国的权力。政府 应该以不记名投票的方式实行定期选举。你应有选举权而且所有的选票都应当一视同仁。

第二十二条：你生活的社会应帮助你发展，并向所有人提供文化、工作、社会福利等大部分资源。

第二十三条：你有权工作、自由选择职业、有权获取足以使你本人和家属能够维持生存的报酬。男女 同工同酬，不受任何歧视。所有工作的人有权联合起来保卫自己的利益。

第二十四条：工作日不能太长，因为每个人都享有休息和带薪休假的权利。

第二十五条: 你有权获取所需的一切, 以维持你本人和家属的健康、不致挨饿, 有衣穿、有房住。一 旦在遭到失业、疾病、变故、年老, 或者你的妻子/丈夫去世, 或在其他不能控制的情况下丧失谋生能 力时, 有权享受保障。即将分婏的妇女和她的孩子有权享受特别的照顾和帮助。一切儿童, 无论婚生 或非婚生, 都应享受同样的社会保护。

第二十六条: 你有上学的权利。人有权上学。初级阶段教育应该是免费的。你可以学一门专业或技 能, 或者按自己意愿继续学习。在学校, 你应能开发你的才能。你应被教会与人相处而不论他的种 族、宗教或背景。你的父母有选择孩子接受哪类教育的权利。

第二十七条: 你有权分享社会的艺术和科学及其产生的福利。你作为一位艺术家、作家或科学家, 创 作的作品应得到保护，并且你应从中获利。

第二十八条: 为确保你的权利得到尊重, 必须建立一种地区的和国际的秩序,在这种秩序中,你的权利能 获得保护。

第二十九条：你对社会负有义务。法律应当保障你的人权，让每个人都尊重他人并得到充分的尊重。 第三十条：任何国家或世界上任何地方的任何个人无权采取任何旨在破坏本宣言所载的任何权利的 行动。 
时间：45分钟

材料: 黑板、粉笔

准备工作：完善 “可能享有不平等社会权力的人群” 分类表, 尽量涵盖所有与你所在社区相关的群 体。活动开始前先完成第一步

概述：学生确认并思考社会权力、特权、与歧视之间的关系

目的: 引导学生讨论社会中权力配置问题, 明确掌权与特权、歧视和压迫之间的关系, 帮助学生 把这些问题与自己的生活联系起来, 增强其分析、解决问题的能力

\section{活动3}

权力、特权、平等

\section{步骤}

1. 绘制一个两栏的图表，第一栏中标注 “较大权力/特权”，第二栏中标注 “较小权力/特权”。引导学 生讨论权力、特权和平等在他们自己生活中和在社会中的概念。

2. 从 “享有不公平社会权力的人群” 列表中读取第一个例子 (穷人和富人)

3. 向学生提问: 哪一人群应归入 “较大权力” 栏，哪一人群应归入 “较小权力” 栏? 提醒同学，人群分 类并没有一个固定的标准。

4. 将学生的回答记录在相应的栏目中。以相同方式完成列表中其他几个例子。（讨论民族、种族群体 或宗教团体时，可列举当地的实例。)

可能享有不平等社会权力的人群 :

穷人/富人 男人/女人 异性恋/同性恋 老板/员工政治家/社区成员 公民/难民 健全人/残疾人

非少数民族/少数民族大众宗教群体/少数宗教群体 富有魅力的人/缺乏魅力的人 
5. 向同学提出以下问题 :

- 看一看 “可能享有较多特权” 列表中, 你能找出自己归属的某一群体吗? 你是否认为这一人群在 社会中享有更多权力? 用你经历或听说过的简短事例说明一下这种权力上的不平等。（留3-5分钟 请同学写下事例 )

课外作业

思考并书面回答这个问题 :

为促进更大平等、赋予 人人尊严, 你在日常生活中 能做些什么呢?

改编自《帮助青少 年终止暴力一一咨询 师、教师、父母的实用 步骤》(Allan Creighton and Paul Kivel. 1990,1992. Alameda,CA:Hunter House Inc.).
- 看一看 “可能享有较少特权” 列表, 看是否能找到你自己归属的某一群体? 你是否认为这一人群 在社会中享有较少特权? 用你经历或听说过的简短事例说明一下这种权力上的不平等。(留3-5分 钟请同学写下事例 )

- 如果两个列表中均有至少一个人群使你产生认同感, 请举手。我们中大部分人是否明白享有更多 特权和较少特权分别给人带来何种感受?

6. 请几位同学自告奋勇分别谈谈他们的故事，拥有较少权利或拥有特权人群的故事。（完全遵从自愿 原则 )

7. 按如下方式进行讨论 ( 每个问题请1-2名同学回答 ) :

- 特权较少的群体所受的待遇, 最吸引你注意的是什么? ( 探讨: 非掌权者经常会受到歧视或压 迫。)

- 这种待遇在拥有较少权力人群中会引起何种情绪? ( 将这些情绪列在黑板上, 确保所使用的都是 情绪词，而不是对事实的描述。）

- 列出的情绪或感情, 最引你注意的是什么?

8. 重新阅读 “较多权力/特权”“较少权力/特权” 列表，请同学完成以下活动：

- 考虑平等的概念, 完成这个句子（以第一组一一富人和穷人为例）：穷人与富人之间的平等。这个句 子使你想到了什么? ( 答案不唯一) 
- 以分类表中其他人群为例完成句子: “… 与 ..间的平等。” 尝试使用不同的词语, 或表达不同 的观点。(如果时间允许, 可让多几组人群讨论)

谁记得《人权宣言》第一条? ( 注: 平等权。将第一条写在黑板上: 人人生而自由, 在尊严和权利 上一律平等。他们被赋予有理性和良心, 并应以兄弟关系的精神相对待。）

9. 以讨论下列问题结束本次活动（或请同学课下思考这些问题）：如何才能实现所有人在权利和尊严 上的平等?

时间：40-60分钟, 具体时间据所分析案例数量而定（2或3个）

材料：黑板、粉笔; 案例材料 (可将案例两部分分别写在纸的两面 )

准备工作: 进行此活动前, 先向同学介绍性与生殖健康权利。从后文所举案例或你本国的案例中选取 三个, 将步骤2的问题提前写在黑板上

概述：分析讨论侵犯性与生殖健康权利的真实故事

目的：使学生能够描述我们行使人权的能力将对亲密关系和性产生怎样的影响, 增强其批判思考 能力

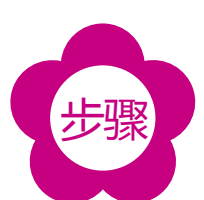

1. 今天我们要讨论性与生殖健康权利的真实案例，请一位志愿者大声读阿莉恰案例的第一部分。

2. 读完第一部分后，请同学在笔记本上写下答案。大声读出写在黑板上的这些问题 :

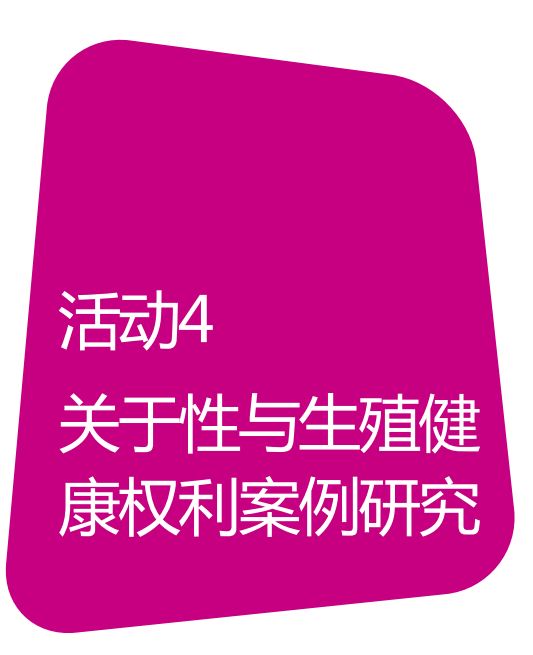


- 对此案例, 你有何感想?

- 此案例涉及哪些性与生殖健康权利?

- 你是否认为这是一种对人权的侵犯吗? 为什么?

- 谁应承担责任? 列出所有应为此事负责的人。

3. 每个问题请一两位同学读出自己的回答，并请其他同学评论。

4. 请读第一部分的同学继续读第二部分（“发生了什么?" ），并讨论：

- 对此事的结局, 你有何感想?

- 如果受害人很富有 (或是为男性或异性恋者)，结果会有什么不同?

- 为了保护这项性权利, 哪些态度需改变?

5. 以相同的方式分析其他案例 ( 每个案例10分钟)。

6. 在完成所有案例研究，结束这节课前，向同学提出以下几个问题：

- 你是否了解本地发生的类似事件? 其中有哪些权利被侵犯?

- 你是否听说过在我们国家或别处发生的任何种类的性权利受侵犯的案例?

- 要阻止这种侵犯, 人们必须做些什么?

- 关于人权与浪漫私密性生活的关系, 我们能得出什么结论? (将同学的回答的结论写在黑板上) 
阿莉恰

第一部分·阿莉恰的故事

波兰妇女阿莉恰自童年起视力就不太好。她怀李后, 看过很多医生。均被告知怀孕和分娩会造 成不可挽回的视力损害。根据波兰法律, 当孕妇健康因怀孕而受到威胁时, 可以进行流产。但医生 们都拒绝给阿莉恰同意流产的证明。她别无选择, 只能生下孩子。

第二部分 : 阿莉恰发生了什么?

分娩使阿莉恰的视力进一步下降。她已经不能再继续工作, 生活起居和照顾婴儿都要靠他人帮 助。她现在完全依赖于政府援助。阿莉恰的案子被提交到欧洲人权法庭, 法庭裁定: 政府有责任建 立有效机制, 确保妇女在合法的情况下进行流产。鉴于阿莉恰遭受的烦恼与痛苦, 法庭判给她一笔 可观的损失赔偿。

\section{阿米娜}

第一部分：阿米娜的故事

阿米娜是一位有三个孩子的尼日利亚离异母亲。与穆帘默德约会 11 个月后, 他请求与阿米娜性 交, 并答应娶她。阿米娜同意了, 并因此次性行为怀李。然而穆罕默德并没有娶她, 结果阿米娜在 未婚状况下生下一名女贞, 因此受到宗教法通奸罪指控。穆罕默德发誓说他不是孩子的父亲, 并被 释放。但阿米娜被判通奸罪, 处以石块击毕刑罚。她的上诉被驳回, 维持原判, 缓刑两年, 以便她 有时间哺乳她的贞孩。

\section{第二部分：阿米娜发生了什么?}

第二次上诉后，原判撤回，阿米娜被无罪释放。法官认为阿米娜没有得到为自己辩护的足够机 会，然而政府却否认她曾被处以石块击毕之刑。后来阿米娜又再婚了。 


\section{法蒂玛}

\section{第一部分 : 法蒂玛的故事}

11岁的西非女孩法蒂玛偶然听到父母谈论自己受割礼的事。想到姐姐受完割礼回来时痛苦的景 况，她非常害怕。法蒂玛又想起了自己最好的朋友，她因割礼导致多种感染，不得不常常去诊所。 法蒂玛不愿经历周围女孩所经历的痛苦, 因此她乞求父母不要强迫自己受割礼。法蒂玛的父母不理 会她的请求，因为他们认为不受割礼就会嫁不出去。法蒂玛年少不经世事，还不能自己做主抉择。 然而, 法蒂玛的姐姐听说城里有这样一个组织, 专门向当地人介绍女性割礼的危险及其对健康的危 害。于是法蒂玛的姐姐请那里的一位工作人员到家里与父母讨论法蒂玛的情况。

\section{第二部分 : 法蒂玛发生了什么?}

援助工作者使法蒂玛的父母认识到割礼危害女儿的健康，并且可用其他方式来标志成人礼。现 在, 法蒂玛婚姻美满幸福, 她非常感激父母的开明。法蒂玛在当初帮她躲过生殖割礼的组织工作, 指导学校里的女孩应如何与父母讨论割礼。

\section{马修}

第一部分 : 马修的故事

马修是一位有同性恋倾向的美国大学生。一天晚上，在一个酒吧里，两个装作同性恋的年轻男 子主动提出载马修回家。马修跟他们去了, 但他们却把他带到一个偏远的地方, 抢劫他的财物, 还 把他绑在栅栏上，用枪把残忍地打他，折磨他。之后两人离去，不顾马修死活。18个小时后，马修 被一位骑自行车的路人发现时, 他仍被绑在栅栏上, 那位路人一开始还以为是个稻草人。马修当时 还活着，但处于昏迷状态。 
第二部分: 马修发生了什么?

马修头骨粉碎，大脑受到严重损坏。由于伤势过于严重医生无力救治，马修始终没有醒过来， 并于五天后死亡。两个凶手被捕, 被判处无期徒刑。马修案受到全国关注, 激起了人们对犯罪的痛 恨。通过了一条以马修的名字命名的法律, 将仇视同性恋、妇女和残疾人的犯罪行为归入仇恨罪之 列。马修的母亲建立了马修・谢泼德基金会，致力于教育、社区延伸服务和宣传工作，以达到 “用 理解、同情、接纳取代仇恨” 的目的。

\section{拉克希米}

\section{第一部分 : 拉克希米的故事}

尼泊尔女孩拉克希米在12岁时被迫结婚。由于无法承受丈夫家的压迫, 拉克希米逃回家里, 但 父母却逼她返回婆家。拉克希米说: “在一位好心女士的帮助下我又逃了出来。她说她妹妹在尼泊 尔另一地区一家工厂工作, 我也可以去那儿, 只用替那家工厂卖衣服就行了。” 路上她被麻醉, 并 被带到了印度。拉克希米说: “我直到那时才知道自己已经以 15，000卢比的价格被卖了。我拒绝 做性工作者, 遭到毒打。一年来我一直被困在妓院里。后来警察突然搜查妓院, 我这才得救, 并被 送回尼泊尔，那时我已经14岁。

\section{第二部分：拉克希米发生了什么?}

拉克希米回到家里, 却被父母拒之门外。后来她结婚了, 但被检测出艾滋病病毒呈阳性。拉克 希米是在被迫从事性工作中感染病毒, 还是在婚后感染, 这就不得而知了。 
性影 


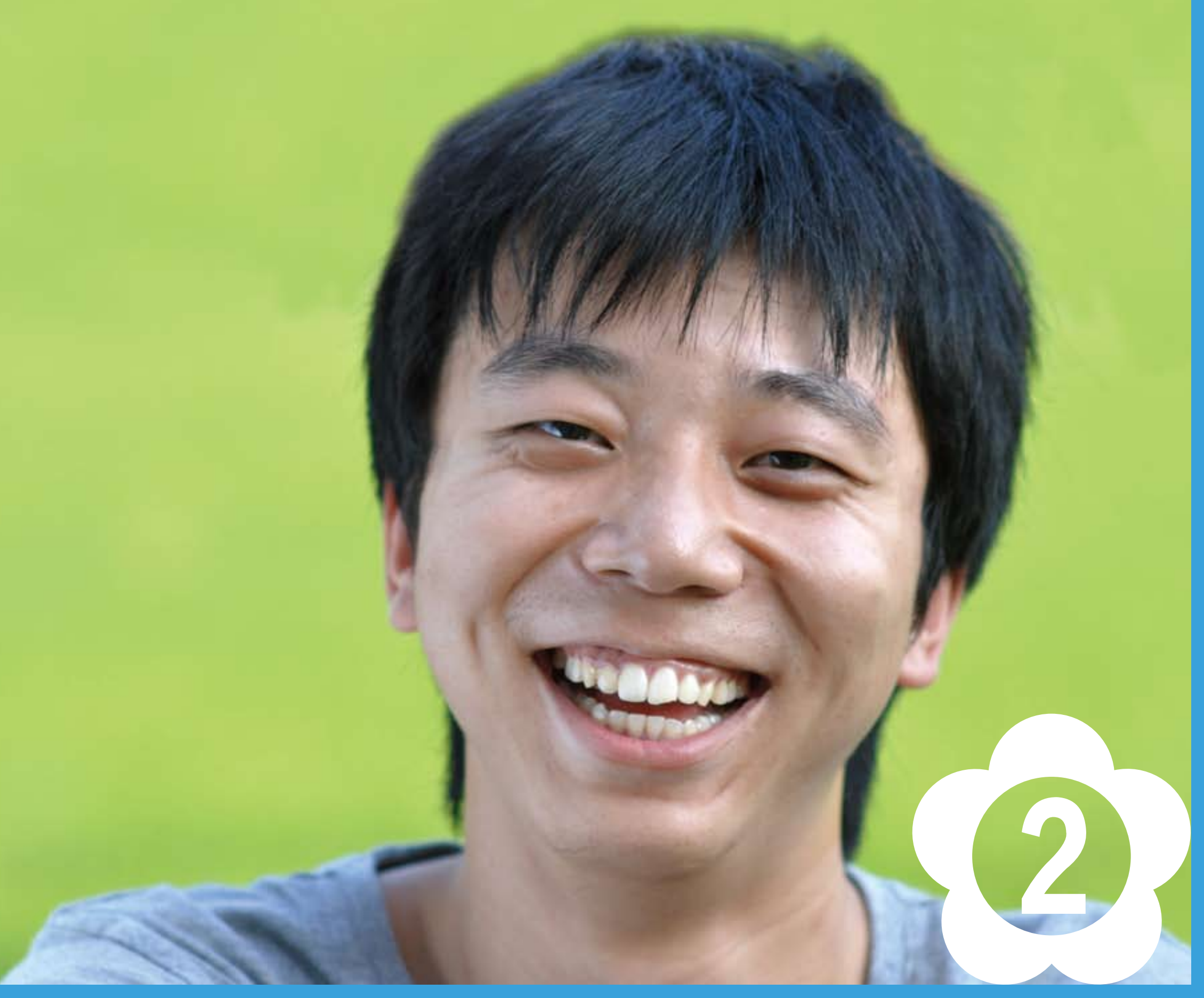




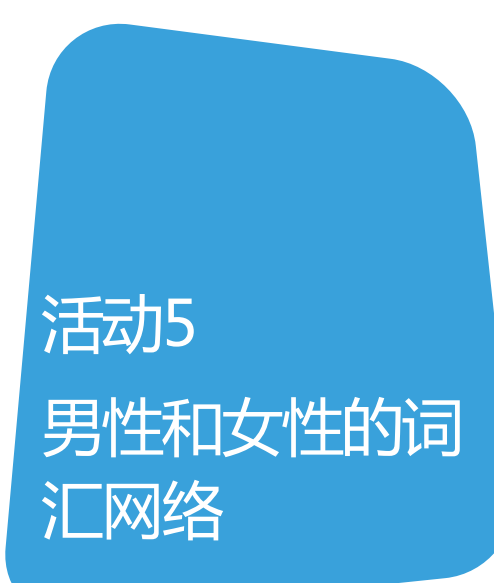

时间：45分钟

材料：黑板、粉笔

准备工作：提前思考并决定你是否需要添加一些适合于当地情况的内容

概述：将学生分组, 将社会对 “男人” 和 “女人” 的理解做成 “词汇网” , 并讨论这些想法的来 源, 向学生介绍社会性别的概念

目的：让学生能够定义 “性别”, 并能区分男性和女性的特征中, 哪些是生理性的, 哪些是社会 性的。增强学生批判性思维和创意写作的技巧

1. 将学生分为4-5个人一组 ( 同性或异性皆可 )

\section{2. 解释:}

- 今天我们将讨论社会性别这一话题, 也就是社会对男女的理解。每个小组将一些经常与 “男人” 和 “女人” 相联系的词汇编织成网络。为了更好地解释 “词汇网” 这个概念, 你或许需要在黑板 上用另一核心词汇做出示范。请参考以 “狗” 为例子的词汇网：狗：可爱的、顺从的、毛茸茸 的、保护的、活跃的、忠诚的、好玩的。

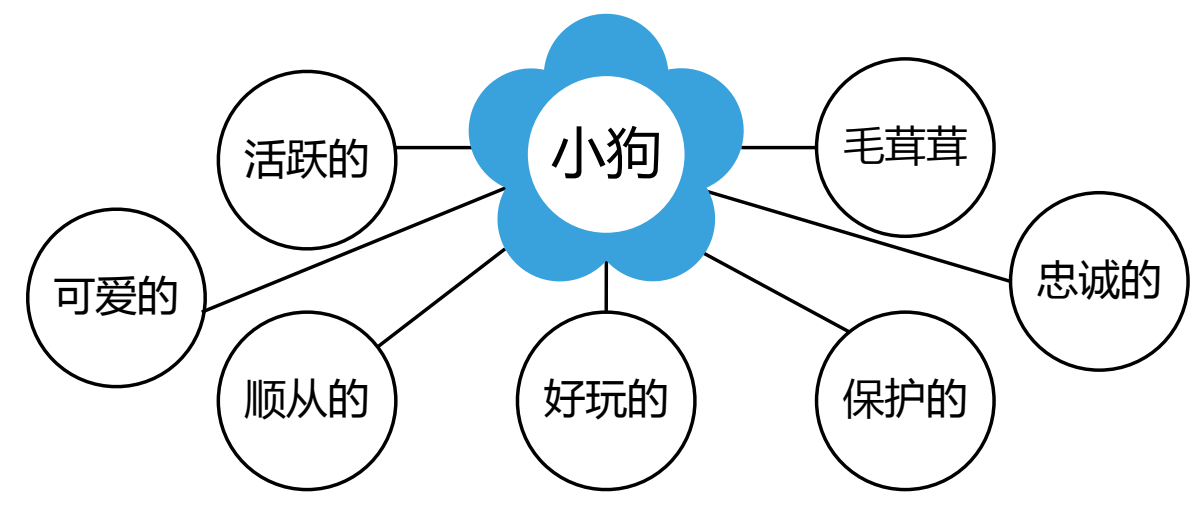


3. 给每小组2-3分钟的时间为 “男人” 和 “女人” 各建立一个词汇网。

4. 将 “女人” 和 “男人” 写到黑板上，并在每个词下方各列两栏，一栏标为“生理性”，另一栏标

为 “社会性", 从一个小组开始, 询问:

- 在你们的词汇网中, 哪个特征是和怎样做个男人相关?

- 这个特征是生理上决定的（生理性）, 还是被社会决定的（社会性）?

- 如果有学生将属于 “社会性” 的特征放到了 “生理性” 的那一栏, 可以通过以下问题纠正他们: 如 果一个男孩或男人没有这一特征, 那他还是男性么?

5. 让每个组的学生依次添加新的特征到黑板上的清单中，直到你得到了所有关于男性的回答。人们从 “身为男人” 联想到的词汇，通常有如下一些例子，请确保学生们的网络中有许多如下的词汇。（为 了得到这些具体的回答，你可能需要抛出很多有启发性的问题。）
- 身体强壮
- 经济成功（很有钱）・骄傲
- 不畏暴力, 也不畏使用暴力

- 情绪不容易外露

- 一家之主

- 强大

幽默

- 在“性”上是主导者

- 酷

○健美

- 对朋友忠实

异性恋

- 父亲

- 勇敢

6. 按照如上步骤，继续讨论 “身为女性”的特征，常见的词汇包括：
- 体贴
善打扮
- 谦恭
- 安静
～情感丰富
- 曲线美
- 顺从
- 善于处理多重任务
- 体能上弱于男性
- 爱聊天
- 务实
- 关怀
- 好的沟通者
- 非暴力
- 母亲 


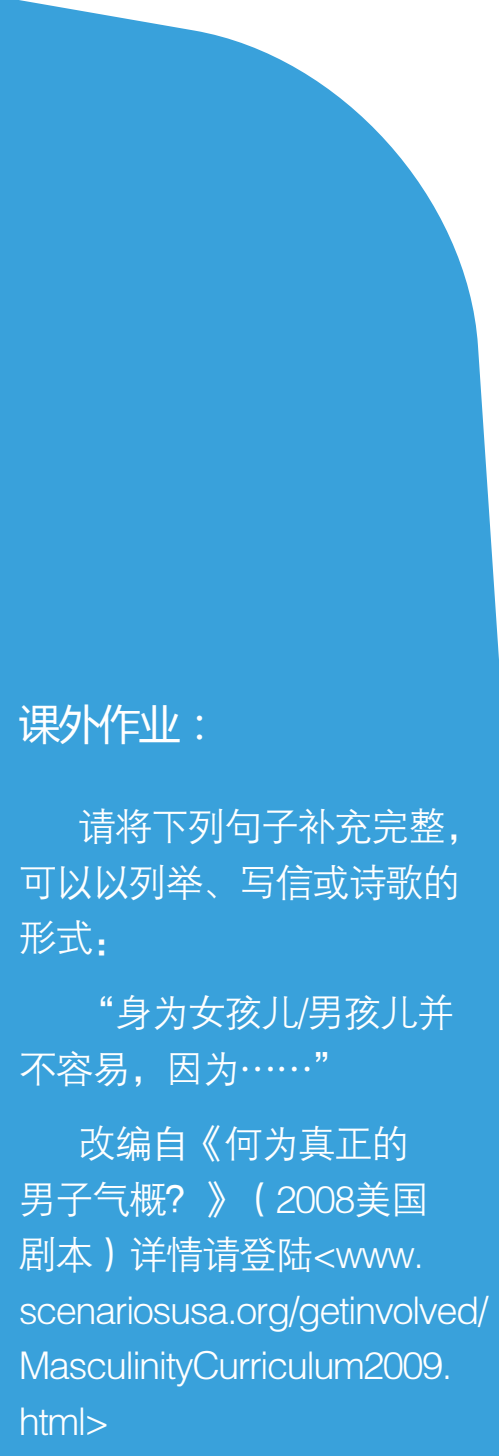

\section{7. 留出10分钟进行小组讨论：}

- 男性和女性的特征有一些是生理性的。例如说只有男性才能成为父亲, 只有女性才能生孩子或 哺乳。

- 但是身为男人或女人的大多数特征都是社会性的一一并不基于生理。

- 社会决定的男性或女性应扮演的角色, 被称为 “性别角色” , 谁曾听说过这个词汇?

- 你对社会上的性别角色有什么感受? 女性被认为该如何举止、如何生活, 你是否同意呢? 男性角 色又该如何扮演呢?

- 你认为性别平等意味着什么?

- 在每一个地区或社会, 都有一些人对性别和性别平等的态度异于常人。

- 社会随着时间推移或地域不同在不断变化, 对待性别角色的态度也是如此。 
时间：45分钟 ( 步骤5可以布置为家庭作业 )

材料：黑板、粉笔

准备工作：在进行活动之前自己先回想一遍这段记忆旅程。记忆活动可能会引发一些学生不好的回 忆, 思考最佳的应对方法, 并思考你可能需要对哪些学生提供更多的支持

概述：学生因为自身生理性别的原因受到某种方式的待遇, 请他们分享这样一段回忆以及当时对 那段经历的感受

目的：使学生能够举例说明, 孩提时代如何获取有关性别角色的信息, 并从个人权利和人权的角 度去思考这些信息, 增强批判性思维的能力

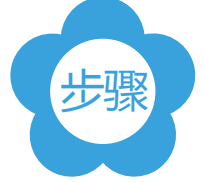

1. 将学生分组，每组四、五个相同性别的学生，请他们带上纸和笔。

2. 解释 :

- 今天我们将探索，作为男孩或女孩分别意味着什么。首先，我们将开启一段深入我们回忆的旅程。 现在请放下笔, 舒舒服服地坐好, 全身放松。

- 现在请回忆自己因为性别而受到某种对待的经历。当你想起什么时, 将它写到纸上。

- 现在, 请回忆一下, 当你受到那种对待时, 心中的感受如何。请写下当时的一些情绪或感受。你可 以选择在小组内分享你的回忆。

3. 在几分钟后, 向学生步㵵:

在小组内, 请花几分钟按你的希望与你的组员分享这段经历或感受, 当然, 分享与否完全由你选择。 
4. 在5-10分钟后，询问：

- 这些经历告诉我们, 社会对女孩和妇女价值持怎样的态度和认识? 男孩和男人呢?

请回想我们曾学习的有关人权的内容, 这些态度和认识对你公平么? 为什么?

5. (注意: 如果你已经超时了，这个步骤可以布置为课外作业) 为了实现性别平等，需要做出哪些 改变? 提醒学生，任何一种情况都可能会有多种结果。给他们改写故事结局的机会，并向他们解 释：

- 回到那些经历中你认为不公平或不正义的片段，不管是你自己写下的，还是在小组内听说的。

- 为这个故事写下新的结局, 一个更为公正的结局。 
时间：45分钟

材料：黑板、粉笔

准备工作: 将步骤1的问题和句子写到黑板上, 或将它们制成一张工作表。思考这个活动可能对某些 学生产生的情绪影响。( 尤其是那些对自己的父亲持有矛盾甚至负面情绪的学生, 或者是 没有父亲的学生。）思考怎样为他们提供能考虑到他们感受的支持

概述：学生将写一写自己的父亲或某位男性监护人。他们将讨论性别角色怎样限制男性扮演父 亲/监护人的角色。他们还将写下自己今后想成为一个怎样的父亲, 或者期望自己的孩子 （或他们所认识的孩子）今后有一个怎样的父亲

目的：使学生能够评价男性监护人的经历和价值, 增强他们的个人反思和写作技巧的能力

1. 通过如下解释来引入话题 :

- 今天我们将讨论父亲的角色以及男性角色的模范。

- 我希望, 在开始时, 你们大家能写一封信给自己的父亲、男性监护人、或你生活中另一个典型的父 亲角色。你不需要分享这封信的内容。但请选择如下一种开头:

亲爱的父亲，你肯定以为我已经忘了，但事实上我记得……

亲爱的父亲, 我需要你…… 


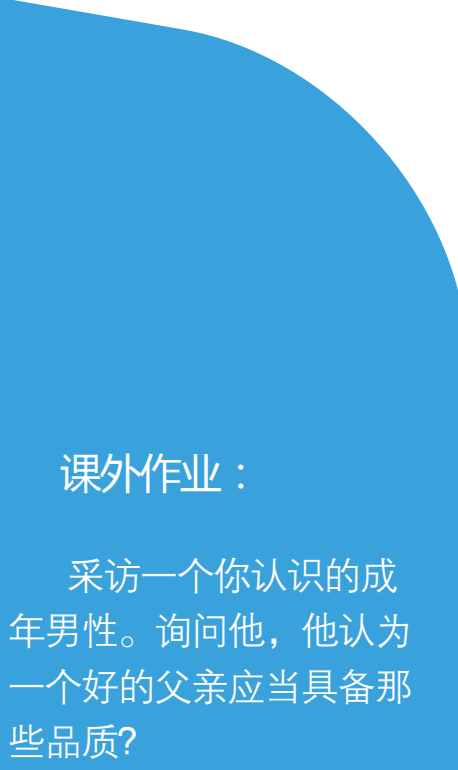

一个好的父亲应当具备那
2. 大约5分钟后，询问：

- 你的父亲、男性监护人或生活中典型的男人是一个怎样的人? 写下至少10个形容词来描述他。你 同样不需要分享这些隐私内容。

- 现在, 列举出 10 个你希望看到的关于父亲这个角色的形容词, 也就是你认为一个很棒的父亲所具 备的东西。

- 看一看你所列举的第二个形容词清单。在可以用来描述绝大部分父亲的形容词上画上勾。将那些 特殊的形容词，或者只能形容一小部分父亲的词汇上画上圈。

- 男人们觉得传统的男性角色有助于还是阻碍他们扮演一个孩子们所希望看到的父亲角色?

- 你是否能想出一个父亲的形象, 他的做法挑战了传统的性别角色?

3. 总结并提问 : 你希望将来父亲的形象发生怎样的改变? 
时间：步骤1-5: 40分钟 步骤6: 4-5小时 步骤7-8: 90-120分钟

材料：黑板、粉笔、复印的讲义、胶带

准备工作: 获得学校对学生调研的许可, 将步骤2中的各个课题写到不同的纸张上 (如果需要的话, 可以调整清单中的课题, 使之与你学校的背景相匹配）, 思考如何在步骤3中将学生分派 到不同的课题组, 为每个学生复印一份讲义

概述: 学生进行校内性别角色的研究, 并报告他们的发现。这个活动要求课外调研和课堂时间相 结合

目的：使学生能够评价男性监护人的经历和价值, 增强他们的个人反思和写作技巧的能力

\section{活动8}

研究项目：

学校环境中的性别

\section{步骤}

1. 在教室的不同角落或地方用胶带贴上写有研究课题的白纸。

2. 解释道 :

- 你们将于下周进行一项调研。需要研究解答的问题是 “学校如何强化或挑战了传统的性别角色?”

- 4 个调查研究小组将从下列课题中任选一个: 教室行为、校外活动、课程和课本, 以及学校统计数 据。

- 试着找出两个吸引你的话题。

4. 让同学们传看《研究步骤》, 确保每个人都明白如何操作。

5. 向每小组分发复印的讲义，让小组讨论他们将如何开展研究。需要时可为各小组提供更多的指导。 
6. 给同学们最多两周的时间进行调查研究，并在需要的时候继续提供帮助。

7. 在调查研究阶段结束时，请学生们介绍自己的研究及其发现。每个展示可用 15 分钟，在展示之后， 询问其他学生：

- 你对这个小组有任何问题吗?

- 这个小组的研究有什么让你特别感兴趣, 或觉得惊讶的东西么?

- 你是否同意他们的结论? 如果不同意的话, 为什么?

- 你还有别的建议么?

8. 在每小组都展示完毕后, 问全班：

- 学校环境怎样强化着传统性别规范?

- 它又怎样挑战传统性别规范呢?

- 为了让学校在性别问题上更加公正, 使其对学生来说更加受欢迎、受尊重和安全, 有什么地方需 要改变? 列举其中5种。

- 你要怎么做才能促使这种转变的发生? 


\section{课堂活动材料}

总体研究说明 :

1. 在小组内认真思考材料上的研究问题。

2. 讨论如何以最佳方式找到问题的答案。如有需要, 请向教师寻求帮助。

3. 讨论决定小组成员分工, 是负责不同的问题, 还是合作研究某几个问题?

4. 收集能回答问题的所有信息。如果需要的话请寻求帮助。

5. 一旦收集到数据, 则按下列内容写作研究报告:

- 你们试图回答的一个或几个问题;

- 研究方法 ( 你们是如何开展研究的);

- 结果 (你们了解到的内容) ;

- 结论（你们对于结果的思考以及得出的结论）;

- 建议。( 可以使学校环境在性别问题上更公正, 并使得学校对男孩女孩来说都更受欢迎, 受 尊重及安全。)

你们小组也将根据研究报告完成一个15分钟的展示。请规划这个展示, 并确保所有的小组成员都参与 到展示当中。 


\section{(小组一)}

你认为, 我们的教科书反映了某种性别倾向, 还是在促进性别平等?

\section{示范问题：}

- 我们的教科书是如何刻画男孩女孩、男人女人的形象的?

- 在插图中, 有多少是女性, 多少是男性?

- 女性在历史、思维、科学、文学和艺术上的成就有没有被充分地体现出来?

- 在教科书的故事和文学作品中, 谁掌握着主动权, 谁是附属者?

- 书本反映的是谁的观点? 书的作者是男性还是女性?

- 性别的刻板印象通过怎样的方式被反映, 被强化或者被挑战?

- 教科书中还反映了对哪些其他群体的刻板印象?

研究方法:

找到至少两本小学或中学使用的课本, 比如社会科学、历史或语文。分析课本中的内容和图像, 并回 答上述作为示范的问题。 
(小组二)

教室环境怎样强化或挑战传统的性别角色?

示范问题：

- 谁在课堂上发言更多, 男孩还是女孩? 谁发言的时间更长? 谁经常打断他人发言? 班级中的头儿是 男孩还是女孩?

- 教师如何改变或强化性别行为模式?

- 有没有学生因为某种刻板印象而受到喜爱, 或被人讨厌? 比如说, 因为他们的种族或性别。

研究方法:

观察几个班级, 最好是不同教师的班级。在观察前, 先进行小组讨论如何 “测量” 和记录你们所看到 的现象。可以做一张简单的表格以供观察时填写。如果有一手表的话, 你们可以测出不同的学生, 男 孩和女孩, 讲话时间的长短。 


\section{(小组三)}

性别规范如何影响学生们对课外活动及使用学校设施的选择?

\section{示范问题：}

- 男孩和女孩是一样地参与到所有课外活动中的吗? 如果他们倾向于参加不同的活动, 哪些活动会 吸引更多的男孩, 哪些吸引更多的女孩?

- 总体来说, 即使他们参加的是不同的活动, 男孩和女孩都一样乐于参加课外活动吗?

- 不管是男孩的活动还是女孩的活动, 学校是否平等地提供相关设施 (公则、体育馆、场地等 等）、装备、服装和供给?

- 对于那些男孩和女孩共同参加的活动, 谁更有可能会获得领导角色?

\section{研究方法}

列出学校中学生课外活动的清单, 如体育运动、学生会、俱乐部等等。观察这些活动, 采访参与其中 的男孩和女孩，并采访这些活动的指导教师。 
(小组四 )

学校总体的性别均衡性如何? 统计数据显示些什么?

示范问题：

- 教师们大多数是男性还是女性? 校长呢? 男教师还是女教师更容易被提升为校长?

- 在数学老师中, 男老师和女老师的比例一样吗? 文学呢? 艺术呢?

- 谁教最低年级? 最高年级呢?

- 学生的情况如何? 男女孩招生规模相同吗? 如果不相同的话, 为什么?

- 是不是所有课程男女孩参与的可能性都一样? 他们是不是同样受欢迎?

\section{研究方法:}

学校校长能为你提供大部分你所需要的详细的性别信息。如果不行的话, 尽可能多地与教师聊天, 询 问他们的学生或者同系其他教职工的情况。如果可能的话, 也可以搜集关于性别规范影响教育机会和 成就的国家数据, 包括不同性别所受的教育程度。 


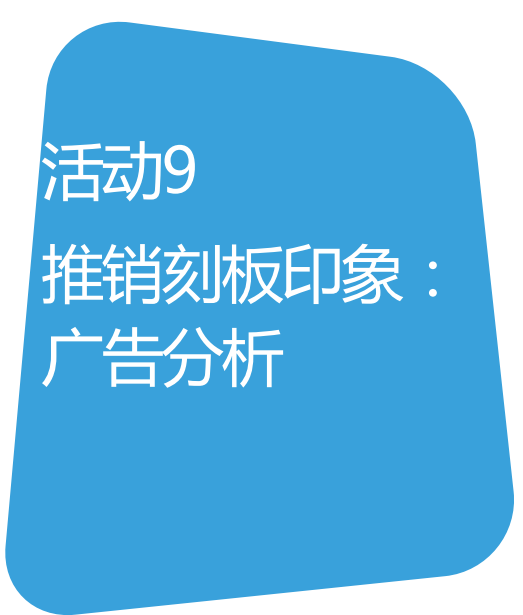

时间：40分钟

材料：黑板、粉笔、带有广告的报纸杂志、剪刀、大白纸、胶水或胶带

准备工作：搜集足够的广告、杂志和报纸，或请学生自己带上用于剪裁的杂志

概述：学生们将搜集拼贴广告中出现的男性和女性形象, 分析广告中关于性别的信息, 并创作一 个能够打破性别固有、刻板印象的广告

目的：使学生能够描述和辨别众多平面媒体在创造和维持性别固有、刻板印象上扮演的角色。增 强学生批判性思维的能力

1. 分发杂志并解释 :

○天我们将来做一个拼贴画一一将杂志上的关于男性和女性的插图剪下来, 并用胶水或胶带粘到 一页白纸上。

学生将制作两份拼贴画, 一份是女性的形象, 一份是男性的形象。

2. 给学生10-15分钟的时间完成拼贴画，并介绍接下来的环节：

- 从男性形象的拼贴画开始，在笔记本中列出广告上男性所具备的特征。

再看女性形象拼贴画, 重复上一步操作。

3. 在黑板上画两个大大的有重合的圆圈。一个标上“男性”，另一个标上“女性”，重合部分标上 “共有”，问道:

- 哪些特征只有在男性的广告中才能见到? 将他们列到 “男性” 的圆圈中。 
- 哪些特征只有在女性的广告中才能见到? 将他们列到 “女性” 的圆圈中。

- 哪些特征在男性和女性的广告中都有出现? 将他们列到 “共有” 的重合部分。

- 从图中你有何发现? 这些广告提出哪些关于女性样貌、行为标准的观念? 男性呢?

- 这些形象如何创造或强化性别的刻板印象? 向女性或男性推销怎样的价值观?

- 这些形象和现实情况相比有何异同?

- 在哪些地方, 媒体形象变成了固有、刻板的印象? 媒体是否创造新的固有的、刻板印象, 或只是一味 的重复和强化社会上既有的固有而刻板的印象? 为什么?

- 年轻人如何被媒体刻画的形象所影响? 你是否曾经看完杂志广告或电视商业广告后, 觉得自己不够 好? 当感觉到广告冒犯、或攻击广告中描述的形象时, 人们会怎么做?

课外作业：

设计一份替换广告, 它打破关于性别固有而刻 板的印象。（如果可能的 话, 请学生将他们的作业 挂在墙上, 或者向同学们 展示。) 


\section{活动10 性别化的空间}

性、性别、艾滋病和人权教育统一行动活动手册

时间：步骤1-5: 45分钟 ( 如果让学生制作自己的地图并标注自己的位置, 时间会更长)

步骤6-7：45分钟

材料：黑板、粉笔; 胶带; 五、六份本社区的地图, 你可以提前画好并复印（或供高年级学生制 作地图的大白纸），彩色铅笔或钢笔

准备工作：回顾一遍社区地图，并做出相应调整使之能够真实反映你的社区

概述：学生将为自己的社区画地图, 并标出适宜不同性别人群的地点。他们将讨论市民生活如何 受到性别的影响。(注意: 教师可以决定本活动与该社区相关与否 )

目的：使学生能够分析性别如何影响公共场所的流动性、安全性和可及性。能指出至少三种性别 角色影响女孩和妇女参与公民生活的方式。增强学生抽象思维能力, 以及应用地理空间描 述数据的能力

1. 向学生解释他们需要画一张社区的 “性别地图” 。从这张地图可以看出在家之外，哪些地方或空间 主要是男性活动场所（聚会，玩乐，或安全地参加其他活动）; 哪些地方主要是女性活动场所；哪些 男女共同活动场所。

2. 将学生分成小组 (不分性别)，并将社区地图分给每个小组。（对于高年级的小组，可以给他们额 外的时间画自己的地图。）

3. 给学生三种不同颜色的铅笔或马克笔，以及一张写有如下地点的清单。( 可以让高年级小组自己列 出自己的地点清单。） 
地点清单：

$\begin{array}{ll}\text { 食物超市 } & \text { 夜间的城市/乡间街道 } \\ \text { 做礼拜的地方 } & \text { 咖啡吧或茶室 } \\ \text { 学校 } & \text { 运动场/篮球场 } \\ \text { 社区中心 } & \text { 酒吧 } \\ \text { 图书馆 } & \text { 火车站汽车站/机场 } \\ \text { 电子游戏室 } & \text { 市政大楼 } \\ \text { 电影院 } & \text { 洗衣的场所 ( 自助洗衣店或河边 ) }\end{array}$

4. 描述本活动 :

- 在小组内讨论, 这些地点主要是男性专属还是女性专属? 亦或者是男性女性共有的场所? 思考这些 地点是否是安全友好的。

- 为男性专属的地点选择一种颜色的铅笔或马克笔, 此后为女性专属的地点选另一种颜色的笔, 为男女皆可去又安全的地点则选择第三种颜色的笔。在地图上用相应的颜色标注出这些空间的 “性别”。

- 完成后, 讨论这幅地图所表明的本社区公共空间的流动性、安全性和可及性。

5. 在学生标注地图和进行讨论时, 在他们中间转转。

6. 请学生将自己的地图挂到墙上向全班展示本组地图，或让他们自行走动查看每幅地图。 
56

性、性别、艾滋病和人权教育统一行动活动手册

7. 询问整个班级 ( 将他们的答案列到黑板上) :

- 你们观察到了什么? 女孩是否有和男孩一样的流动性? 在公共场所的可及性上, 女孩和男孩有怎 样的对比?

- 公共场所的安全性对女孩男孩是一样的吗?

男孩长大时, 这些情况会改变吗? 女孩呢?

产生这些差异的常见原因有哪些?

- 你对公共场合的被 “性别化” 有何看法?

- 为了更积极地参与公民生活, 人们需要哪些途径到安全的公共领域当中?

- 对女孩和妇女开放的有限的安全公共空间如何影响她们的公民能力? （询问学生, 他们认识的女 性中, 拥有公共决策权的有多少? 相比男性, 有多少女性有公共权力? )

男孩和男人是否同样受到影响? 为什么是? 为什么不是? 
时间：步骤1-7: 45分钟; 完成任务 (在课堂中或课外作业)：2-3小时 步骤8：45分钟

材料：白纸、马克笔、针线、胶带、订书机, 或制作书的其他工具; 关于 “创造一本属于自己的 书” 的材料

准备工作：检查学生材料中 “女性活动家” 的清单, 确保这些女性领袖都适用于本项培训。寻找足够 多的女性领袖, 以确保每3人或4人小组都有不同的研究对象。如果可能, 复印课堂活动材 料。考虑联系一所小学, 鼓励学生访问该小学并向孩子们朗读他们制作的书籍

概述: 学生设计一本有关女性领袖的书。( 注: 该活动需要利用图书馆或网络, 因此, 你必须按 此准备。1

目的：帮助学生了解女性领袖, 并将其勇气和成就与个人生活进行联系和比较, 增强研究技能

\section{活动11}

女性活动家：创作 一本属于自己的书

\section{步骤}

1. 分发相关材料 (或者将内容写在黑板上)

2. 将学生分组，每组3至4人。并向他们解释：

- 今天我们将开始一个活动, 了解社会公正方面的女性领袖;

- 每组需研究一个不同的女性领袖生平;

- 每组需撰写一篇与该女性领袖有关并可供孩子阅读的故事, 并将该故事制作成一个

有封面的儿童读物。（向学生们说, 你会安排将他们所制作的读物借给附近一所小学的学生, 或 念给他们听。 ) 
3. 查阅 “女性活动家” 的清单 (见附后的课堂活动材料 )，向他们解释如何选择女性领袖，避免重 复，并提问：

- 有没有人曾听说过这些女性领袖人物中的任何一个?

- 学生可以提出别的女性领袖, 但必须提前向教师说明, 以确保该女性符合本活动的标准。

4. 查阅《指导手册》的问题 ( 见附后的课堂活动材料, 或者让学生将问题抄写在黑板上)

5. 解释：

1 ) 学生需要在课堂或课外完成该任务;

2 ) 需要多少天完成该任务并上交成果;

3 ) 从哪里获得有关女性活动家的信息。

6. 向学生解释当任务完成时，组中的每一个成员需要负责整个任务中的某一方面：撰写故事；编辑校 对；制作插图；设计完成封面；书籍装订 ( 缝合，粘合，钉书 )

7. 用余下的时间让各组选定要写的 “妇女活动家”，并在组员间分配制作读物的多项任务 (参见附后 的课堂活动材料 )。教师应走动以便协助

8. 展示完成的书籍: 每组需在所有学生面前大声朗读书籍中的故事，如果可能，也可以选择孩子作为 听众。阅读之后，提问：

- 你们最佩服的女性人物是谁?

- 你是否觉得自己也能做与这些女性领袖相似的事情?

- 如果想达到这个目标, 需要做什么? 
课堂活动材料

女性领袖：

Rigoberta Mench ú 危地马拉人权领袖

Rosa Parks 美国民权领袖

Mukhtaran Bibi 巴基斯坦反性暴力领袖

Aung San Suu Kyi 缅甸政治领袖

Wangari Maathai 肯尼亚环保领袖

Michelle Bachelet 智利政治领袖

Arundhati Roy 印度作家

Sophie Scholl or Martha Wertheimer 反抗纳粹主义领袖

Hillary Clinton 美国政治领袖

Vera Ngassa or Beatrice Ntuba 喀麦隆法官

Louise Arbour 前联合国人权高级专员

撰写一位女性领袖书籍的参考问题：

1. 她的名字叫什么?

2. 她早期生活的重要经历?

3. 她观察到哪些社会问题? 她是怎么开始关注这些问题的?

4. 你如何了解到她关注了这个问题的? 
5. 针对该问题，她做了些什么?

6. 有其他人帮助过她吗?

7. 讲述一个关于她身上展现出某种你所钦佩的品质的故事。该品质可以是勇敢、坚定、忠诚、耐心、 具有创造力、聪明、和他人相处融洽、对于未来的远见等。

8. 描述她的行动产生的社会影响。

9. 如有可能, 引用一句她所说过的名言。

10. 为你的书起一个书名, 这个书名不要只是她的名字, 而要包括她所关注的问题或者你从她身上学到 的品质。

将小组成员的名字填在空格之中：

撰写人（也可以多人）：

编辑:

校对;

插图:

美工:

装订: 
时间：45分钟

材料：黑板、粉笔

准备工作：想想在你生活的环境中, 人们改变外貌的方法以及哪些方法会引起健康问题。检查列举的 清单并且思考哪些做法适合给学生讨论

活动12

对外表的改变

概述: 学生找出不同文化中男性与女性的理想外貌, 审视人们为了达到符合该理想外貌通常的做 法, 并讨论这些行为所引发对他们健康方面的后果

目的：质疑人们为了符合文化界定的所谓 “理想” 外貌而承担的压力; 描述人们, 尤其是妇女和 女孩, 为了尝试达到这种外貌而付出的努力以及该努力会引起的不良后果; 增强批判性思 维能力

\section{步骤}

1. 通过提问以下问题来介绍话题:

- 女孩男孩觉得哪些压力使他们需要拥有某种外貌?

- 对于我们中的大多数, 达到理想的外貌是现实的还是不切实际的?

- 如果人们没有理想的外貌, 会有什么样的感觉?

- 人们为了让自己 (或者自己的孩子 ) 达到具有吸引力的理想外貌, 通常会做些什么? 例如, 头发? 皮肤? 或者身材?（将学生的回答写在黑板上。）

2. 从下表人们改变外形的措施中挑选一二进行讨论：

增肥或剧烈节食; 极端的塑身; 晒黑或者美白; 使用化妆品, 美甲; 打耳洞, 或者纹身; 剪发或者 
接发; 拉直、粱头发或者染发; 美容手术; 女性割礼，男性割包皮。 3. 提问 :

- 你如何看待上边提到的做法? 哪些是有趣的或者只是一种自我表现，哪些是有害的?

- 来自其它文化背景的人们对于这些做法会有什么感觉?

- 人们固有观念中理想的外貌来自哪里? 在追求理想外形方面, 女性是否比男性承担更多压力? 在 此过程中, 得利的是谁? 遭到了伤害的又有谁? 这些压力会如何影响人们的自尊心?

- 停止这些具有伤害性的改变身体行为有多重要? 你希望哪些做法可以停止?

- 在你成长的过程中, 是否有任何人告诉你这些行为是危险的并且劝你停止?

- 你有没有和其他人分享过你对这类伤害性做法的了解, 或是你的感受?

- 针对此事, 你会做些什么? 


\section{3}

时间：90分钟（可以分为两部分）

材料: 黑板、粉笔

准备工作: 思考或者阅读自己身边存在的暴力现象。需要注意, 由于涉及个人经历或者认为这是 私事, 所以对大多数人来说, 讨论家庭暴力是一件困难的事情, 不要强迫学生发言 学生创作一部短剧, 反映亲密关系中的尊重和暴力, 并讨论在他们自身的关系中如何促进 活动13 亲密关系：从暴力 到尊重 概述：尊重

目的: 让学生讨论如何对待亲密关系中的暴力, 以及如何促进一段建立在相互尊重之上的亲密关 系; 增强创意写作和表演的能力

\section{步骤}

1. 严肃地介绍该话题。并解释：

- 在该活动中, 我们将讨论分析人们有时会在亲密关系中使用的各种暴力;

- 学生会有15分钟准备一个短剧, 反映一段亲密关系——对已婚夫妇、未婚男女, 或者同性伴侣;

- 学生的短剧将有助于我们思考如何确保亲密关系是建立在相互尊重的基础上的。

2. 将学生分为 4 组 (或者 4 组以下，根据总人数而定），每组 5 或 6 人。

告诉其中两组: 准备一个短剧（少于5分钟）, 表现一段包含暴力的亲密关系, 可以是身体上或者心理 上的暴力。尽量贴近生活，用你所见或者所闻的事例作为原型; 
告诉另外两组: 准备一个短剧（少于5分钟），表现一段彼此尊重的亲密关系。展示这两个人如何以非 暴力的方式处理冲突或不同意见。

3. 教师在各组准备的过程中不断走动，提供相应的帮助

4. 从包含暴力的短剧开始，让每一组展示剧情。在每个短剧结束后，要求观众提问，并讨论是什么性 格特点或者相互关系导致了暴力

5. 依照同样的步骤表演关于彼此尊重的短剧，并讨论是什么性格特点或相互关系促成了健康和非暴力 的亲密关系

6. 基于以下问题，组织一场讨论：

- 在一段亲密关系中, 有哪些形式的暴力? （例如：控制、强迫、叫喊、威胁以及身体上的暴 力。)

- 暴力关系具有哪些特征? (在黑板上写下标题 “暴力关系” , 并在下边列举其特征。)

- 为什么很多人, 尤其是青少年对于家庭暴力感到无能为力? 他们是否真的无能为力?

- 一个健康和谐的关系具备哪些特征? 确立一段彼此尊重的关系, 必备条件有哪些? (在黑板上写 下标题 “彼此尊重的关系”，并在下边列举其特征。)

- 短剧中的事例是否贴近现实? 在你的日常生活中, 是否也看到过这样的场景? 短剧中表现健康关 系的事例是否贴近现实? 在你的日常生活中, 是否有这样的关系?

- 身体上的暴力是否主要是男性施与女性? 女性是否对男性实施暴力? 你觉得这些暴力的成因是什 么? 当你看到这些形式的暴力, 你一般会怎么做? 你能做些什么?

- 你认为建立彼此尊重的亲密关系可能吗? 对于建立健康的关系，作为个人我们能做些什么? 
时间：步骤1-4: 1小时 步骤5-7: 1小时

材料: 黑板、粉笔

准备工作：让学生事先了解针对女性暴力的一些知识

概述: 在小组范围内, 学生制定消除针对女性暴力的策略, 并将其以电视新闻的形式表现出来

活动14

反对性暴力

目的：让学生最少列举两个反对针对女性暴力的策略, 增强解决问题和在公众场合演讲的能力

1. 将学生分为4组或5组，要求一半的小组准备一段新闻报道，另一半的小组准备一场给政府的演讲。

2. 提问：“谁应对停止针对女性的暴力负责 ?"

3. 解释:

- 设计一些减少针对女性暴力的策略或项目;

- 你将有40分钟的时间准备一场5-10分钟的演讲展示（一段新闻报道或者针对当地或国家政府的演 讲展示, 由你所属那个小组而定）;

- 你的演讲应假设听众已经了解针对女性暴力的问题。你的任务是展示那些积极的措施来应对该 问题;

- 强调有哪些措施已经在人们的日常生活中得到了实施, 或可以被实施; 有哪些措施已经在当地组织 或社区实施了，或可以被实施; 以及有哪些措施国家政府或联合国机构已经实施，或可以被实施;

- 同样应考虑如何让成年男人和男孩参与到这一努力中去。 
对于针对地方或国家政府的演讲, 需解释: 你将告知政府官员, 组织或个人已经采取了哪些行 动, 解释政府应如何回应。一些学生可以扮演政府官员, 另一些学生可以扮演专家或市民。

对于新闻报道, 需解释: 你要尽可能吸引人们的注意力, 并让自己的演讲尽可能简短, 清哳。使 用有趣的故事展示, 针对个人关系中暴力问题的工作是如何开展的。注意你的展示显得有个人色 彩并且生动。

4. 保证每个人明白布置的任务。告诉各组他们有45分钟的时间进行准备。教师在他们准备的过程中四 处走动

5. 在展示开始前，给每组5分钟时间再审议各自的展示，使其更流畅

6. 要求每组进行展示。并记下演讲中的要点

7. 所有演讲结束后，提问 :

- 想出减少针对女性暴力的方法难度有多大?

- 对于与女性工作, 不同小组普遍的话题和想法是什么?

- 对于与男性工作呢?

- 你觉得哪些想法最好? 为什么?

- 是否每个人都有责任努力消除针对女性的暴力?

- 作为青少年, 在家庭、学校和社区, 你可以为消除针对妇女和女孩暴力做点什么? 
时间：步骤1一3: 15分钟课外作业: 1小时 步骤4: 30分钟

材料：黑板、粉笔、采访提纲表格

准备工作: 如果学生没有可接受访问的老人, 设法找到一些愿意接受访问的老人, 为每一个学生复印 一份采访提纲表格

概述: 学生采访一位爷爷奶奶 (或者这一辈的其他长者), 了解他们年轻时的性别角色和规范, 并和自己的经历做出比较, 讨论性别角色是如何变化的

目的：让学生列举至少三点性别角色和规范在一段时间里的变化; 提高研究的技能

\section{步骤}

1. 向学生解释，本活动探究的是，性别规范如何随着时间的推移而变化的

- 你需要单独访问两名我们祖辈的老人（你的爷爷奶奶或者其他老人）, 尽可能邀请和你同性别的采 访对象。如果你不认识任何老人，你的家人或者教师可以帮助你寻找一个可以接受访问的老人;

- 向每个老人提问: 在他们年轻的时候, 男性和女性角色分别是什么样的? 他们认为现在这些角色发 生了什么样的变化? 可以提问的话题包括教育、娱乐、友谊、爱情、婚姻的习俗, 以及工作。鼓励 每一个访问对象尽可能多的讲述他们所希望讲述的事情。

2. 将采访表格画在黑板上, 如果有复印的表格, 分发给所有学生, 告诉学生如何使用该表格:

- 在左列记录采访对象的回答;

- 采访结束后, 在右列写下现在这些性别角色是怎样的。 
3. 确保每个人都清楚了布置的任务，并找到了相应的采访对象。告诉学生应何时完成采访和填好采 访表格

4. 当所有学生完成采访问后，讨论：

- 你们都访问了谁? 和老一辈的人谈起性别角色的话题有什么样的感觉? 谈这些话题时, 有什么让

- 你或者他们感到不太舒服或者困难的地方?

- 他们所说的哪一点让你最吃惊? 根据采访, 你认为性别角色是一成不变的, 还是可以变化的?

- 在生活的哪些领域, 性别角色和规范的变化最大? 你认为, 哪些变化是好的, 哪些是不好的? 为 什么?

- 导致变化的因素很多。全球化的媒体如何影响性别角色? 科技和网络呢? 领袖人物的行动呢? 推 动社会公正的草根运动呢?

- 你认为在下一代中, 性别角色还会继续变化吗? 会在哪些方面变化呢?

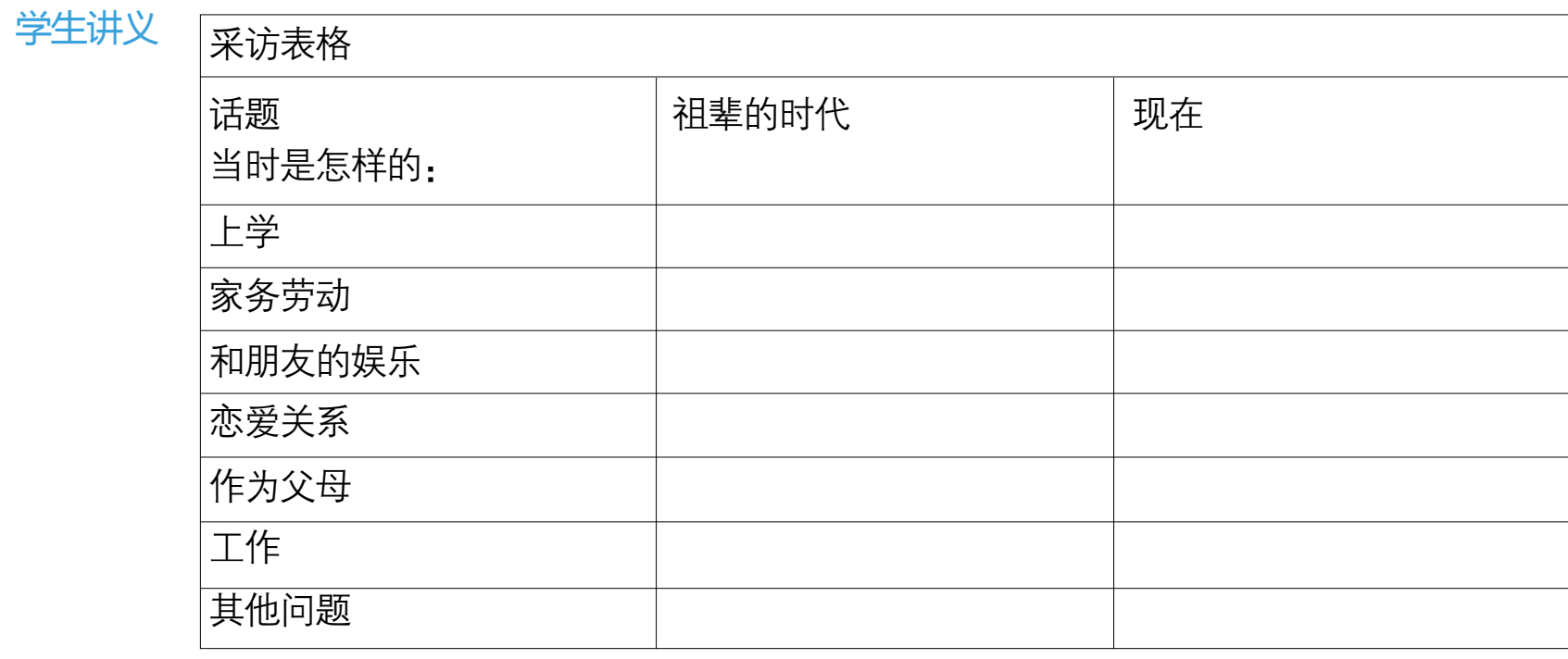


时间：步骤1-7: 45分钟 步骤8: 45分钟

材料：一些歌曲, 抒情诗或诗词的范例

准备工作: 教师先熟悉学生中流行音乐。在步骤4, 可考虑是否加入一些合适的剧情。鼓励学生认真 考虑他们自己的剧情并将其描述出来。可以选择一些学生作为评委 (参见步骤6 )

活动16

说真话的力量

概述: 通过创作歌曲和诗歌, 学生练习如何主张性别平等。在活动开始之前, 每一组需至少完成 本书中活动1、3、5或6中的一项

目的：帮助学生找出并分析男女关系中的权力不平等

\section{步骤}

1. 解释该活动将探索性别角色有时如何造成了人际关系中权力的不同。提问：

- 在性别角色和平等方面, 我们都学到了哪些东西?

- 性别角色对于人际关系中人们是否拥有平等的权力, 可能产生什么影响? (用5至10分钟时间 作答）

2. 向学生说明，他们将通过创作诗歌或歌曲来探讨这个问题。他们可以用说唱音乐或其他任何形式

3. 将学生分为两人一组 (同性或异性)，并且让他们和各自的搭档讨论如何分工，例如，一起创作表 演,或一人负责创作一人负责表演

4. 学生们可从以下的例子选取材料, 以此创作歌曲或诗歌

- 一个男孩为他的同性恋朋友辩护 
- 一个男士告诉他的朋友, 他无权强迫他的妻子和他发生性关系;

- 一个银行家向一个不情愿的男士解释, 为什么要贷款给他的妻子 (或者妻子解释为什么她需要并 理应拥有自己的钱）;

- 一个年轻女孩面临来自中年男人的性骚扰;

- 一个丈夫坚持要求他的妻子完成包括做饭、照顾孩子在内的一切家务劳动, 尽管他们都有全职的 工作;

- 一个女孩因为被卖到性服务行业而在回到家乡后遭遇羞辱和歧视;

- 一个年轻的妻子因为嫁妆太少而受到丈夫的惩罚;

- 一个男孩因为比较女性化而受到其他男孩嘲笑;

- 一个女性试图说服自己的丈夫或男朋友使用安全套;

- 一个男孩在他的爸爸对妈妈实施家庭暴力时保护妈妈;

- 一个不同的观点: 前联合国秘书长安南曾说, 我们明白该做些什么来应对艾滋病的传染, 它需要 真正积极的改变, 这些改变会给予妇女和女孩更多的权力和信心, 并改变社会各个阶层中男性和 女性之间的关系;

- 学生可以有自己的想法, 但需要提前获得教师的同意。

5. 给学生一些如何完成该任务的指导意见：

- 用几分钟的时间和你的搭档讨论一下你们想写哪个情景;

- 用余下的课堂时间创作歌曲或者诗歌;

- 思考在这些情景中, 不同的人会有怎样的争论, 而他们又会用什么来证明自己的观点。 
- 思考一下, 如果你是故事中的人, 你会有怎样的心情。他或她会怎样用语言来表达他们的心情。

- 记住, 这不是一个笑话, 也不应轻视暴力。

6. 告诉学生他们需要展示各自作品的日期。可以请3至4名同学担任评委，选出他们最喜欢的表演，你 也可以选择那些对表演不满意的学生当评委

7. 给同学们25-30分钟的时间创作他们的歌曲或诗。如果需要，也可以让他们以课外作业的形式完成该 任务

8. 当展示开始时（也可单独占用一个课时），召集评委，并确定挑选优胜者的标准 (例如，信息传达 得最好、演得最好、最好的歌曲、最好的诗等 )。请学生开始表演

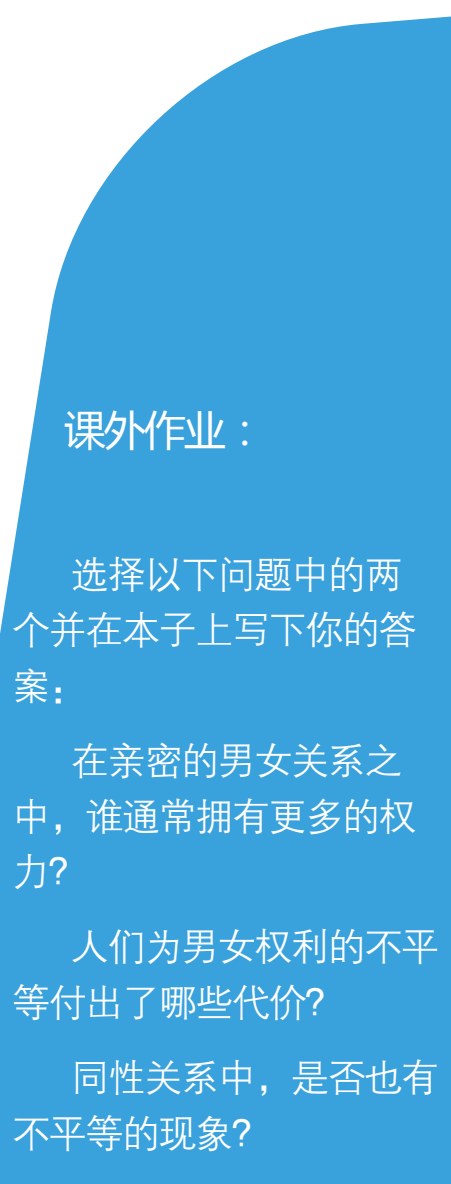

要想在亲密关系中争 取更大平等, 可以做些什 么? 
性 


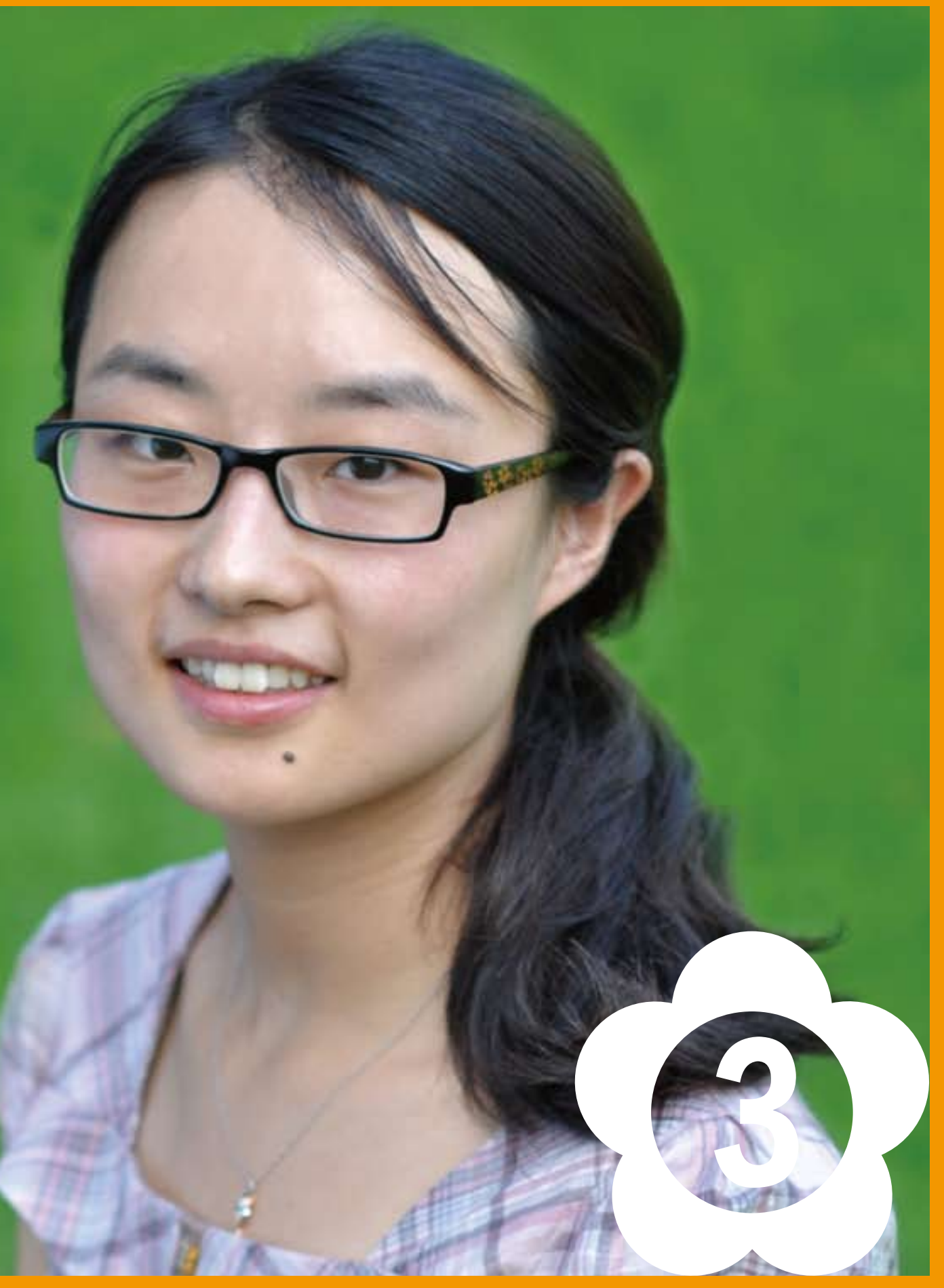




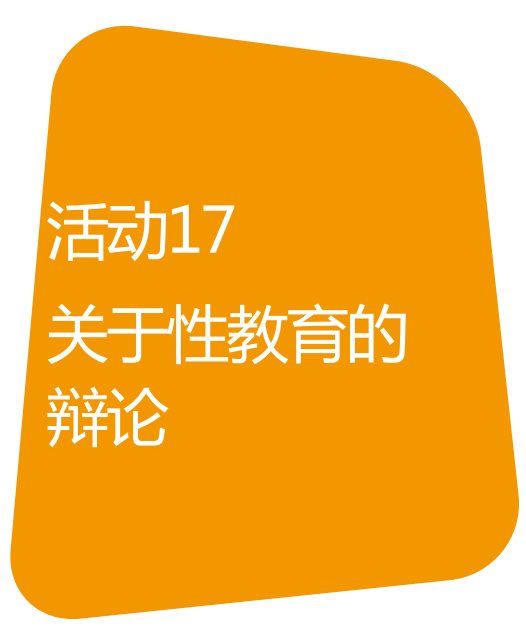

时间：45分钟

材料：黑板、粉笔

准备工作：思考辩论如何进行, 仔细复习下一页中给教师的信息, 条件允许的话, 可以邀请另一位教 师作为裁判来观察整场辩论 ( 判断哪方赢) 。如果没有邀请到教师, 挑选两名学生和你一 起来做裁判, 准备一份给获胜方的奖品

概述: 学生们就这个题目开展辩论: 青年人应该学习有关性、性别以及生殖健康方面的知识, 还 是应该只学习怎样远离婚前性行为

目的：让学生来描述关于性教育的政策, 并思考自己的观点。增强他们分析和演讲的能力

\section{1. 用如下方式引入活动}

- 我们倾向于把性看做是非常个人和私密的事情。但是在现实生活中, 性的许多方面都成为了公众 话题。例如，许多立法者、政客还有普通公民都在就性与艾滋病教育进行争论。

- 今天我们也来开展一场关于性与艾滋病教育的正式辩论, 我们将会准备并陈述双方的观点。

2. 将人员分为两组, 如果没有一个外来裁判的话, 那你就从学生中邀请两名志愿者, 让他们不参与任 何一组，而是帮助你评判整场辩论

3. 介绍辩论的题目: “性教育学校应该教育年轻人避免婚前性行为，而不应教授有关安全套、避孕的 知识，或者两性知识。" 
- 一组将会对辩题持赞成态度, 另一组则持反对意见, 也许你不同意被分到的这一组的观点, 但还是 应该站在本方持有的观点上思考, 尽力找出让人信服的所有理由。同时也想出支持另一方的证据和 观点。辩论陈述中要阐述所有方面;

- 选两名代表来陈述你方观点（如果是男女混合的组，应挑选一男一女）;

- 第一发言人各自有3分钟做开场白, 你的队友将会帮助补充你的论点。第二发言人将有3分钟回应 对方的陈述观点, 所以一定要认真听对方一辩的陈述并做好笔记, 以便做出回应;

- 然后我们会有一些时间让刚刚没有发言的同学提出问题并进行回答: 双方队员们可以发表点评, 也 可以给对方辩友提出问题，对方辩友中没有发过言的同学可以进行回应;

- 每队将有10分钟时间讨论和准备陈词。辩论结束后, 将有一队会赢得此场辩论, 应该依照哪一队 的在陈词准备上做得较好来挑选优胜队伍, 而不是建立在裁判同意哪一方的观点这一基础上。

5. 公布正方反方队伍, 给每个队伍10分钟发言准备, 教师可不时参与到他们的讨论中, 并确保每一个 人都参与到了其中。如果你邀请了两位学生当裁判，可以用这个时间来告诉他们在辩论时计时，并在 时间快到时给予提醒。教师提供他们一些裁判辩论说明，例如，注意听发言者给出的有力的论据

6. 让每队的第一个发言人进行开场白式的观点陈述 (每人3分钟；一共6分钟) 然后第二个发言人将对 于对方发言人提出的观点进行回应和反驳 (每人3分钟；一共6分钟)，最后，让队伍中的其他成员来 提问题和评价（6分钟），快到时间时提醒两队做最后简短的总结陈词

7. 教师与裁判商讨并决定出获胜队伍，和学生裁判一起对两队的表现给出积极具体的反馈意见，告诉 他们在辩论中表现突出的方面

8. 在宣布获胜队伍后, 讨论:

- 你们中间有多少人被分到了与自己观点相符的队伍里? ( 请举手示意) 哪些被分到了与自己观点不 相符的队伍里? (请举手示意) 为自己不同意的观点来辩论, 是容易还是困难? 
- 关于性的各个方面中, 是否只有性教育成为了一个公众话题呢? 社会是不是对性的许多方面都有 所控制? 还有哪些关于性的问题约束着人们的行为, 例如法律、风俗、和社会规范，（探讨：性 暴力; 在是否许可性行为的问题上, 对男孩女孩的不同标准（“双重标准” ) ; 女人在公共场合 必须掩蔽身体 (在一些情况下) ; 男孩有要使自己变得性活跃的压力; 对同性恋的差辱或者犯罪 化谴责）；

学校应该教育学生要避 免婚前性行为, 而不应该 教给他们有关安全套、避

孕、或两性关系方面的知 识。正确还是错误?

很多国家都在进行有 关性教育的争论, 那些认 为学生只应该学习远离婚 前性行为的人常常认为, 教给年轻人过多的关于安 全性行为的知识, 会鼓励 他们比没有这种知识时更 早发生第一次性行为。但 是, 通过调查, 全面的性 教育并不会提前第一次性 行为的时间。那些认为有 必要进行全面性教育 (包 括安全性行为) 的人相 信, 这些知识是帮助年轻 人在性生活上做出健康安 全决定的工具。

- 是否每个人都同意主流文化对于性的态度? 有人选择根据别的态度或观点来生活吗? 是否每个地 方对于性的态度都是一样的, 还是有区别? 这些态度是一成不变的, 还是在不断改变? 那么性还 仅仅只是一个私人问题吗? 还是它也是一个社会普遍关注的问题? 


\section{时间：40分钟}

材料：黑板、粉笔，给一半的学生复印 “玛雅的故事”，给另一半的学生复印 “拉苏尔的故 事”，提供足够大的场地，使得在两组进行讨论时不会相互干扰

准备工作: 准备工作: 准备好解释什么是形容词。思考如何让讨论在相互尊重的氛围下进行, 要准备 应对一些当地的不尊重俚语的使用, 特别是描述那些性生活活跃女孩的词语。复习《活 动指南手册》第三单元中有关性行为双重标准的信息。可登陆网站<www.popcouncil.org/ publications/books/2010_ItsAllOne.asp>

概述：学生们对故事中的角色是男性还是女性进行假设, 来考察自己对该角色行为的反应

目的：让学生们意识到我们对于一个人做出的假设和判断常常是基于他们的性别的。增强他们的 批判性思维

\section{步骤}

1. 解释:

- 今天我们要阅读和讨论一则有关青少年的经历、感受和面临的挑战的故事;

- 我们将分为两组; 每组将会阅读一则故事, 讨论你们的观点, 并做记录。

2. 把班上的人分成两组。一组发 “玛雅的故事”一组发 “拉苏尔的故事”。(对于故事不要做任何解 释），给足够的时间让他们阅读、回答问题，并在各组内对回答作出比较。让两组离得足够远，以确 保他们不会听到彼此的讨论 
3.15分钟之后，让拿到 “拉苏尔的故事” 的小组简略的阐述一下他们对于故事相关问题的答案，不要 让他们解释或者讨论他们如何回答的理由。将他们答案中的一些关键词写在黑板上 ( 形容词、名词符 号 )

4. 让另一小组做同样的事情

5. 在写着有关 “拉苏尔” 的词汇列表上方写上拉苏尔的名字，在写着有关 “玛雅” 的词汇列表上方写 上“玛雅” 的名字

6. 告诉大家这两个故事其实是一模一样的，只是改变了主人公的性别，然后提问：

- 从对于拉苏尔和玛雅的反应对比中我们观察到了什么?

- 有关男性标准和女性标准的对比, 告诉我们一些什么?

- 社会中的女孩是否受到这个双重标准影响?

- 你对于这个双重标准怎么看?

学生阅读材料

玛雅的故事

玛雅的第一次性行为发生在16岁。因为她从来没有过一个男朋友，她的两个朋友有时会因此取笑她。 他们鼓动玛雅去主动邀约一个男孩并且和他发生性关系。玛雅感到很好奇, 但更多的是来自她朋友的 压力, 所以她邀约了那位男孩, 他们发生了性行为。学校里几乎所有的人都知道了这件事。

在接下来的两年里, 玛雅和四位不同的男孩发生了性关系, 一个是学校的同学, 一个是她的邻居, 另 一个是她在工作中认识的, 还有一个是通过朋友认识的。她十分享受性爱的过程。玛雅知道她自己并 不会与其中任何一个结婚, 但她对这些男孩都很好, 也从不对他们撒谎。拥有性伴侣使玛雅觉得自己 
很有吸引力并很重要。

大多数时候, 玛雅都小心地使用了安全套, 但她还是不小心怀孕过一次, 并做了流产, 还有一次她不 小心从一个性伴侣那里感染了性传染病, 不过马上治疗好了。

现在玛雅和一个小伙子订婚了, 她的家人对他很满意, 她也很喜欢这个小伙子, 但她知道, 她必须要 等到两年后与他结婚了才可与与他发生性关系。有一次她去另一个城市看望自己的表姐, 恰巧遇到了 她曾经的男朋友, 他想和玛雅再次发生性关系, 玛雅觉得在结婚前只发生这一次性关系并不会伤害到 任何人。她想, 毕竟两年是很长的一段时间。事后, 她向她最好的朋友吐露了这个秘密, 而她的朋友 表示了理解。

读完这则故事后，思考以下问题，然后在小组内讨论：

1. 你们觉得玛雅的同龄人会怎么样看玛雅? 她们会给她一个什么样的标签来形容她呢?

2. 你怎么看待玛雅?

3. 写下最少三个或四个你认为可以形容玛雅的形容词。例如, 她是快乐的还是不快乐的? 自信的还是 没有安全感的? 是否是诚实的? 是否是有吸引力的? 是否是值得尊敬的? 是不是典型的女孩? 是不是有 道德的?

\section{拉苏尔的故事}

拉苏尔的第一次性行为发生在16岁。因为他从来没有过一个女朋友，他的两个朋友有时会因此取笑他。 他们鼓动拉苏尔去主动邀约一个女孩并且和她发生性关系。拉苏尔感到很好奇, 但更多的是来自他朋友 的压力。所以他邀约了那位女孩并且他们发生了性行为, 学校里几乎所有的人都知道了这件事。 
在接下来的两年里, 拉苏尔和四位不同的女孩发生了性关系, 一个是学校的同学, 一个是他的邻居, 另一个是他在工作中认识的, 还有一个是通过朋友认识的。他十分享受性爱的过程。拉苏尔知道他自 己并不会与其中任何一个结婚, 但他对这些女孩都很好, 也从不在感觉上对她们撒谎。拥有性伴侣使 拉苏尔觉得自己很有吸引力并很重要。

大多数时候, 拉苏尔都很小心地使用了安全套, 但他还是不小心让一个女孩怀孕过一次, 她做了流 产, 还有一次他不小心从一个性伴侣那里感染了性传染病, 不过马上治疗好了。

现在拉苏尔和一个姑娘订婚了, 他的家人对她很满意, 他也很喜欢这个姑娘, 但他知道, 他必须要等 到两年后与她结婚了才可与与她发生性关系。有一次他去另一个城市看望自己的表哥, 恰巧遇到了他 曾经女朋友, 她想和拉苏尔再次发生性关系, 拉苏尔觉得在结婚前只发生一次性关系并不会伤害到任 何人。他想, 毕竟两年是很长的一段时间, 事后, 他向他最好的朋友吐露了这个秘密, 而他的朋友表 示了理解。

读完这则故事后，思考以下问题，然后在小组内讨论：

1. 你们觉得拉苏尔的同龄人会怎么样看拉苏尔? 他们会给他一个什么样的标签来形容他?

2. 你怎么看待拉苏尔?

3. 写下最少三个或四个你认为可以形容拉苏尔的形容词。例如, 他是快乐的还是不快乐的? 自信的还 是没有安全感的? 是否是诚实的? 是否是有吸引力的? 是否是值得尊敬的? 是不是典型的男孩? 是不是 有道德的? 
时间：45分钟

材料：黑板、粉笔, 复印足够的判断关于性欲正误的练习题（或者将题目写在黑板上）、答案 准备工作：审议练习题并作必要调整。确保你有揭示答案的所有信息

概述：学生完成一次有关性欲和性别的判断正误练习

目的：让学生能够辩识有关性欲正确的信息

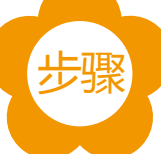

1. 告诉学生:

- 我们今天要讨论性欲这个题目, 我们将解除大家对于这个主题普遍存在的疑问;

- 首先, 我们先完成一次判断正误的练习。

2. 将练习题发下去, 让学生快速的填写

3. 请一位志愿者来念出第一道题目，然后用如下问题来鼓励大家进行讨论：

- 你们中间有多少人认为第一道题目是正确的?

- 谁觉得是错的?

- 如果没有人的回答是正确的, 问一些问题来帮助他们找到正确答案。只有在没有人正确答出时, 教 师才公布答案。向学生确认是否还有问题或意见。

4. 对每道题都重复进行这个步骤

\section{活动19}

关于欲望的真相 


\section{5. 留下最后的10分钟来提几个问题：}

- 有哪些言论是你们开始时觉得正确, 但后来发现只是误解?

- 这些错误的信息会给我们对于自身和性的感受带来什么影响?

- 你注意到了社会对于男性性欲和女性性欲的说法是有区别的吗?

- 你觉得这些普遍存在的错误信息会给性关系带来什么影响?

- 你认为为什么我们对于男性和女性性欲得到的看法会有不同, 该不该如此呢?

\section{课堂活动材料}

\section{有关性欲的练习题：哪些是正确的?}

阅读每一道题目, 判断正 $(T)$ 误 $(F)$, 将你的答案写在右边的格子里

1. 大多数女人其实并不渴望性。

2. 如果你渴望性，你就应该去发生性关系。

3. 社会期望女性不应该渴望性，这个观点会影响她们对性的渴望程度。

4. 如果一个男孩勃起了, 就表示他想要发生性行为了; 如果一个女孩的阴道湿润了, 就表示她想要发 生性行为了。

5. 有些女人的性欲比她们的性伴侣还要强烈。

6. 人们对于一些他们在真实生活中并不想经历的东西会有性幻想。

7. 对于意外怀孕和被感染性病的恐惧会影响性欲。 
8. 每个人的性欲程度会随着时间和环境不断改变。

9. 如果你是一位男性, 你随时都想要发生性行为。

10. 大多数老年人仍有性欲。

11. 如果一个男人在性爱过程中无法勃起, 表示对方并非他想要的人。

12. 一些药物会影响性欲。

13. 一个人可以对自己不爱的人产生性欲。

14. 男人有时不渴望性也是很正常的。

15. 如果你渴望性, 你会变得性兴奋。

\section{教师参考答案}

\section{性欲练习题的答案}

1. 错误 大多数女人是渴望性的, 但是, 如果女人无法从性中得到乐趣, 她有可能对此失去兴趣。

2. 错误 性欲并不一定要联系到性行为。事实上, 人们可以很享受对性欲的感觉而不是去想要发生 性行为。

3. 正确性的渴望会受社会期望的影响, 如果一个女人认为她不应该渴望性, 她可能就会压抑并否认自 己的性欲。

4. 错误 男人勃起或女人阴道变得湿润可以发生在没有任何原因的情况下, 例如, 大多数男人早上起床 时都会勃起, 因为他们的膀胱是满的, 处于青春期的男孩常常会在没有任何性欲或性兴奋的情况下自 然地勃起。 
5. 正确 性欲程度因人而异, 也同样会受到环境的影响, 在一些情侣中, 或生活中有些时候, 一个女人 的确有可能比她的性伴侣更渴望性

6. 正确幻想就是幻想, 一个人可能并不想真的体验幻想中的情节

7. 正确 不同的情绪, 例如害怕, 可以影响人们对性的渴望。

8. 正确对性的渴望的 “正常” 程度差别十分大, 性欲受身体、情绪和社会因素影响, 如果一个人的性 欲对她或他造成了麻烦, 可以向性健康专业人员求助。

9. 错误 男人也并非时刻都渴望性, 他们对性的渴望程度是不断在改变的, 所有的人都是这样。

10. 正确性欲也许会随着年龄的增加而减少, 如果社会并不赞许老年人的性行为, 他们的性欲可能因为 差耻之心而减少，但也有许多人终生都会有性渴望。

11. 错误一个男人在性爱中没有勃起可能有多种原因, 可能是由于健康状况（例如糖尿病或心脏病）、 服用了某一类药物, 或因为情绪原因 ( 例如因为性爱表现而产生的焦虑紧张, 或者对他人或替他人感 到愤怒，或者是因为走神）。

12. 正确 有些药物的副作用确会导致性欲的下降, 但有些则会帮助男性勃起, 例如伟哥。医生通常不会 与病人讨论药物对于性的影响，如果对这种副作用有担心，可以向内科医生或者药剂师咨询

13. 正确人们常常会混淆性欲和爱, 你可能爱一个人但并不渴望与她或他发生性关系, 你可能也会有对 一个你不爱的产生性冲动。

14. 正确没有人，不管男人还是女人，会无时无刻的想要发生性行为。

15. 错误 有的时候人们会渴望性, 但却不会被激起性兴奋, 这种情况有时会发生在大多数人身上, 这没 有什么值得担心的。 
时间：35-40分钟

材料：黑板、粉笔

准备工作: 通读整个活动, 思考如何在尊重、理解的前提下讨论社会中少数群体的话题

概述：学生找出与周围人相同和不同时内心的感受, 并肯定少数群体包括 ( 性少数群体 ) 的 权利

目的：让学生能够体会与其他人不同时的情绪; 肯定所有人的人权，包括在性少数群体的人权。 增强情感反应和对话交流能力

1. 按如下步骤介绍这个话题:

- 思考你觉得自己与在坐的大多数人都很相似的两点（除了年龄）;

- 现在思考你与在坐的大多数人都不同的两点。内容并不需与人分享。只要你认为是真的便可;

- 用1分钟思考一下, 与周围人相同的两点带给你怎样的内心感受, 拿出你的笔记本, 并写下四个描 述你感受的情绪性词汇;

- 接下来, 再想想你与其他人不同的两点, 这让你有什么感觉? 写下四个描述自己感受或情绪的 词汇。 
性、性别、艾滋病和人权教育统一行动活动手册

\section{2. 引导关于以下问题的讨论：}

- 不必说出是什么事让你感到与其他人相同或不同，只说出，能与大家相同，你有什么样的感受? （在黑板上的一栏中写下这些词汇）

- 与大家不同又使你有哪些感受? (在黑板上的另一栏写下这些词汇 )

3. 提醒学生们，每个人都会有不同感受，或者在某方面“不合群”。有时有这种感受并非不正常。留 出15分钟的时间给大家讨论下面的问题:

与他人不同的感受在什么时候起到积极作用? 与众不同可以带给我们哪些好处?

- 什么时候与他人不同给我们带来的是消极影响?

- 那些被认为与大多数不同的人容易被怎样对待? 你怎么看? (探讨：这种对待对吗? )

当你觉得与周围人不同时，你希望被如何对待? 那些与大众不同的人（并未对任何人造成伤害） 也拥有与所有人相同的权利吗? 这与他们如何不同有关系吗? 
时间：45-50分钟

材料：黑板、粉笔、复印足够多的 “同性恋的成长经历” 的案例研究

准备工作：做好充足的准备, 以便能够回答有关故事和主题的一些问题, 确保你的学生会严肃认真 的对待这个主题, 并且你也可以很轻松的教授这一主题。如果需要的话, 可以向一些性多 元化的专业机构寻求帮助, 或从其他可靠的渠道寻找信息。如果使用 “男同” 和 “女同” 这样的词汇在这里会有不好的言外之意, 则考虑使用别的没有恶意引申含义的词汇代替

概述: 学生们阅读一些青年人发现自己是同性恋的故事, 然后讨论这些年轻人的感受, 经历和反 应。纠正错误的信息

目的：让学生能够描述两个关于青年人发现自己被同性吸引的经历, 感受他们的感受, 并纠正错 误的信息; 增强他们的批判性思维和复述的能力

\section{活动 21} 被同性的人吸引：

个人故事

1. 把班级分成三组。解释:

- 今天我们将要了解年轻的同性恋者, 也就是只被或主要被同性吸引的人的社会经历和感受。你们将 会读到关于青年同性恋者的真实案例;

- 每组都会拿到一个不同的故事, 学生将讨论并写下对三个问题的答案。

2. 将下面的问题写在黑板上, 并大声读出来：

- 故事中的人物讲述了哪些感受? 尽可能多地列出你所发现的感受; 
- 故事中的人物认为同性恋者有什么特征?

- 故事中的人物怎么处理他们的情绪?

3. 将故事材料发下去, 给大家 10 分钟阅读、讨论和写下问题答案。告诉他们等会要与所有人分享他们 写的答案

4. 10 分钟后，请两位拿到尼亚的故事的小组成员来简述一下尼亚的故事（不是读）; 然后陈述他或她 对于这三个问题的答案，将尼亚的感受写在黑板上，接下来，围绕李和毛的故事重复这一流程

5. 再给出15分钟让大家来讨论如下问题：

- 总的来说, 尼亚、李和毛对于自己是一个同性恋者有什么样的感受? 造成这些感受的最主要原因 是什么? 他们是如何意识到自己是男同性恋或女同性恋的? 有什么事情发生在他们身上而导致他 们成为同性恋吗?

- 你读这些故事时有什么感受? 有什么让你感到吃惊的? 你对同性恋者了解多少?

- 社会对于性的多元化的态度和看法在不断改变吗?

\section{学生阅读材料}

\section{尼亚的故事}

我是一个25岁的女性同性恋, 在我小的时候, 我是一个好学生并且有很多的好朋友。当我进入青春期 时, 我感到自己有了很多不同。我会梦到自己轻吻某个人, 虽然看不清那个人的脸, 但是却可以感受 到那个人的身体, 是一个女性的身体。我觉得有些疑惑并开始有些讨厌自己。15岁的时候我突然意识 到自己被一个我认识的比我大的女孩深深吸引了。感觉上这是真正的迷恋。将这和我曾经做过的梦结 合起来, 我慢慢开始意识并接受自己被同性吸引的事实。但我第一次读到同性恋这个词还是在杂志的 
一篇故事中, 说的是一个女警官强奸了一个17岁的女孩。我至今还记得那本杂志是如何描述同性恋 的, 它说同性恋是一种变态并且是一种疾病。还有另一篇文章是关于男同性恋和艾滋病的。两篇文章 都把同性恋描绘成变态和病态。我意识到自己将是被排斥的 “那群人” 中的一员。每个人都想被爱并 成为社会中的一员一一没有人想要被逐离。我想我可能是不对劲了, 我想我是病了。“同性恋” 这个 词在我脑海里成了一个巨大的威胁, 它就像一个不可触碰的禁忌, 我也成为了一个禁忌。我很害怕。 我觉得自己变态和不正常, 为了应对这种情况, 我躲进学习之中, 变成了众人眼里的一个书呆子。

\section{李的故事:}

我是一个23岁的男性同性恋者。我在乡村长大。大约在我11岁的时候, 我开始发觉自己喜欢看男人 的身体。我很惊讶, 也不知道为什么会这样。在我住的地方有一个言行举止都很像女人的男人, 他的 绰号就叫 “姑娘”。我有点怕他, 也知道我不想像他那样。我还记得有一次, 一本关于性的杂志传 到了我们那小镇, 里面有一些关于同性恋的信息。我读了这些内容, 感到有点害怕。之后我搬家去了 城里, 那里我的朋友议论着想和女孩子发生性关系。我向他们撒了谎，说我对一个特别的女孩很感兴 趣, 但其实我脑海里想的一直都是男人。为了掩饰, 我总表现的比其他男孩子更强硬, 并且常常运 动。我希望没有人会猜疑我是同性恋, 也确实没有人这样想。但我恨自己, 因为我不知道为什么我会 变成这个样子。我想, 为什么我不尝试尝试? 于是我与 5 个不同的女孩子约会, 给她们送礼物, 我们常 常出去, 有时会看电影。我不停地努力, 但就是没有办法。我甚至还四次与其中的一个女孩发生性关 系, 但这就是无法满足我。 


\section{毛的故事}

我是一个22岁的男同性恋者。在我成长的过程中，我们有一个同性恋邻居。我的父母也有同性恋朋 友。所以我从小知道同性恋人和其他人是一样的一一就像我的父母, 他们有孩子、工作和宠物。当我 15岁的时候, 我意识到, 我对我最好的朋友本诺的感情超越了对一个朋友的喜欢。我不知道如何告诉 他, 所以我拜访了我妈妈的同性恋朋友。他和他的同性恋伴侣在一起生活了20年。首先他说, 他知道 这需要很多诚实的反思才能发现我们到底是谁。然后, 他问我本诺的态度, 并鼓励我对本诺诚实。但 他解释说, 本诺可能和我想的并不一样。事实上, 本诺也很有可能因此远离我。第二天, 在足球练习 后, 我告诉了本诺我对他的的感觉。起初, 他很不高兴, 并告诉我说, 他不认为我们可以继续做朋友 了。我感到十分沮丧。但几天后本诺来到我家, 他告诉我, 他对我或对任何男性都没有感到性吸引, 但他仍然希望和我成为朋友。我很高兴我的朋友又回来了, 即使我们之间永远不会发生浪漫的情节。 作为一个少年的同性恋总是不容易的。我经常遇到的一些人, 他们对我的生活方式感到不舒服。我知 道我很幸运能有这样一个支持我的家庭, 许多其他年轻的人就没有。这也就是为什么我现在在一个专 门帮助那些质疑自己性取向、但并不知道向谁求助的年轻人的热线里工作。 
时间：步骤1-4: 45-50分钟 步骤5-6:（完成, 并且如果你准备了问答部分）10分钟

材料：黑板、粉笔; 给每个组发一份课堂活动材料; 空白纸片; (如果包括步骤6 ) 一个袋或一 个大的信封; 盒子; 问题答案

准备工作：查看材料中的陈述确保你能够解释它们为什么是对的或者错的。决定活动是否要包括5、6 步骤。如果要包括5、6步骤, 则确保一个可靠的信息来源, 以便回答书面的问题

概述：学生们在游戏中判断出关于性行为的表述是否正确, 并在讨论中纠正错误的概念

目的：让学生们识别关于性行为的正确信息

\section{步骤}

1. 告诉学生们他们将讨论关于性行为的一些普遍的错误说法，并阐述正确的说法

2. 把学生分成两队, 在黑板上写一个积分表, 为每队划出一栏。将资料分发出去并作出解释:

- 各队要逐一讨论材料中的每句话是否正确。学生将有7分钟完成表格;

- 最后我们会每个问题过一遍, 各队给出自己的答案。每做对一道题所在队伍加一分。（让他们开 始: 7分钟 )

3. 在检查时, 让一位志愿者大声读出第一个句子。然后问每个队所选的答案，最后阐明正确答案，给 正确的队伍加上一分，询问并解答任何疑惑。对每个句子都这样做一遍。每句1分钟

4. 20 分钟后 (17个句子都结束后) 宣布胜出的队伍。并问他们为什么在性与性行为方面会存在这么多 的误解
活动 22

性行为谬误

和事实 
如果你准备后续问答:

5. 发一些空白纸片, 然后解释:

- 很多人对性与性行为有疑问。可以在纸片上写下自己关于这些话题的任何问题, 不用写名字, 但 要提出比较严肃的问题。没有问题, 可写上: “我现在没有问题。”

结束后把纸片把纸对折;

- 我之后会对所有严肃的问题给出解答。我相信你们不会提交一些笑话、不成熟或者不适宜的问 题，这类问题是不会被解答的。

6. 过几分钟后，让学生们折好纸片，然后将纸片放到一个大的信封或者盒子里。在学生们走后，看一 下这些问题。对那些自己不能确信的问题，从可靠的资源上找答案，以便在解答会上可以给予正确信 息。下节课预留时间，来解答学生们的问题

\section{课堂活动材料：}

\section{性行为一一错或对}

阅读下列句子并决定他们是对 ( T ) 还是错 ( F ) , 在右边的对应表格中标注出来。

\section{1. 自慰是有害的。}

2. 如果女性在第一次做爱时没有血迹，说明她不是处女。

3. 应该由男人发起性。

4. 可以不通过性交满足性需要或者达到性高潮。

5. 频繁自慰是一个问题。

6. 很多妇女只通过阴道性交不能达到性高潮。 
7. 女性第一次性交会感到疼痛。

8. 自慰有助于了解身体对于性刺激的反应。

9. 同性恋想要和同性别的任何人发生关系。

10. 男性性兴奋后不能控制自己而且必须射精。

11. 取悦你的伴侣最简单的方法是和他或她交流他或他喜欢怎么样或者怎样感觉舒适。

12. 通过口交会感染性传播疾病。

13. 许多男性在他们某段性经历时都会遇到不能勃起的问题。

14. 男性阴茎大, 会使他的伴侣会觉得更享受。

15. 如果女性特喜欢性, 就不能相信她会忠诚于她的伴侣。

16. 可以有性行为的合适年龄是 18 岁之后。

17. 男性只要在女性的阴道里进行足够长的性交,就会使她达到性高潮

\section{教师参考答案}

“性行为-错或对” 的答案

- 1 错误 自慰是无害的。恰恰相反, 它是一种了解自己身体的安全而又有益的方式。但是, 它是一种 个人选择。很多人自慰, 但也有些人并不这么做, 而且还有一些人并不赞同这种做法。 
- 2 错误 有一些流血的情况是由于处女膜撕裂造成的。但是处女膜在一些常规的体力活动或运动中 很容易被拉伸或撕扯, 并且也可能被手指或者卫生棉条拉扯张开。处女膜破损或者不流血不能说 明一个女孩已经有过性行为。

- 3 错误 根据许多文化, 男性是性行为的主导, 而女性被看作在性方面应更被动些。但是, 这个模 式反映出来某些文化对性别角色的态度。事实上, 女性也可以主动发起性交。即使那些遵循传统 性别角色的女性，也经常通过间接方式来和她们的伴侣交流她们的性需求。

- 4 正确 做爱可以包括很多方式（抚摸、接吻、按摩、用手刺激）。可以通过性交以外的方式如抚 摸或摩擦等达到性高潮。

- 5 错误 经常性的自慰是没有关系的。只有在一种情况下自慰被视为一种问题: 就是自慰影响到正 常的生活, 或者在自慰过程中妨碍到其他人或者对他人造成了伤害。

- 6 正确 大多数女性不是只通过阴道性交达到高潮, 而是要通过对阴蒂的刺激达到高潮。如果女 性本身 (或者他们的伴侣) 在阴道性交之前过程中或者之后直接刺激阴蒂, 她们会更容易达到高 潮。

- 7 错误 女性的第一次性交可能会痛或不痛, 为了减少不适或疼痛感, 伴侣之间应该互相探索彼此 的身体, 并且在插入之前保证双方性兴奋已充分, 以便使女性阴道充分润滑。如果女性感觉紧张 或害怕, 那么他们需要时间等待。

- 8 正确 自慰是一个人了解自己身体对性刺激反应的最好途径之一, 还可以帮助妇女和女孩了解 如何达到性高潮。

- 9 错误 无论是异性恋者, 同性恋者还是双性恋者, 都只会被特定人性吸引。性吸引是由很多因 素决定的。

- 10 错误一些男性认为, 如果达到真正的性兴奋, 他们就有高潮必须射精, 但这样并不正确。 
中止也许会造成不舒适, 但这种感觉会逐渐减退, 无论男性还是女性, 都可以在一次性经历中 的任何时候中止。

- 11 正确 每个人对于什么是性高潮都有自己的偏好, 相比去猜测你的伴侣的喜好而言, 直接与 他或她交谈, 是更快且值得信赖的方法。交流是拥有积极且令双方愉悦的性关系的关键。

- 12 正确口交可以导致许多性传染疾病的传播, 性传染疾病包括了疮疹、淋病、人乳头状病 毒、梅毒、尿道炎、乙肝、软下疳、艾滋病病毒。

- 13 正确 许多男性在他们生命中的某个时候会有这样的经历, 这是正常现象, 无需担心, 忧虑 会增加复发的可能性。

- 14 错误 大的阴茎并不会在性生活中给女性更多的快感。虽然女性各有不同, 大多数女性都认 为重要的是男性是如何进行性交的, 而非他阴茎的尺寸。事实上, 过大的阴茎可能会导致女性 的不适甚至疼痛。

- 15 错误 享受性是男女天性所致, 一个人对于性的喜好与他或她是否被信任毫无关系。这句话 其实是说女性不应该喜欢性, 这反映了在性的问题上对待男性和女性的双重标准, 男性喜欢性 是可以被接受甚至是被希望的, 女性则不然。这个观点是不公平的, 错误的、且固有而刻板 的。

- 16 错误 对于性行为而言不存在正确的年龄。每个人都会决定他或她什么时候准备好有性行 为, 这取决于一个人的成熟程度、关系好坏、价值观念和自身感受。每个国家对于一个人可以接 受性行为的足够年龄都有法律规定。

- 17 错误 对于很多女性来说, 阴道性交不能使他们达到性高潮, 无论男性持续时间长短。更多 时候女性是因为对阴蒂的直接刺激而达到性高潮的。伴侣之间真诚的交流可以帮助他们找到愉 悦的性活动。 
性、性别、艾滋病和人权教育统一行动活动手册

\section{活动23}

为什么我们做 (或 者为什么不做 ）： 有关性决定的强迫 选择练习

\section{时间：30-40分钟}

材料：黑板、粉笔、强迫选择理由列表

准备工作：删去那些不合适的选择项, 加上有意义的选择项, 在黑板的一边写上 “同意” , 另一边写 “反对”

概述：学生们将被要求去做出这样一些选择：同意或反对以下这一系列进行性交或者是不进行性 交的理由

目的：让学生们能够批判性地去考虑自己或者是他人所作出的有关性的决定。增强思考能力和写 作能力

\section{1. 活动介绍:}

- 今天我们将讨论一些人们决定性交或是不性交的理由。许多不同的环境和感受对人们决定是否进行 性交有影响。有时候人们会有着复杂的感受;

- 为了探讨这个问题, 我将会读一些理由。这些都是一些价值陈述, 对于这些陈述没有绝对的对与 错。如果你同意, 站在同意那边, 如果不同意, 则站在反对那边。即使你的观点是较为摇摆或者中 立的, 你也得在同意与不同意间作出选择。然后, 学生们可以分享他们的观点。（确保每个人了解 这个规则。) 
2. 读出每一条理由。读完后，给出足够的时间让同学们作出判断，然后分别陈述两个观点。即便讨论 没有结束，也要进行到下一条理由。时间允许下，读尽可能多的理由

\section{3. 保留5到10分钟进行总结，提问：}

- 为什么对于年轻人来说思考清楚进行性行为与否的理由是十分重要的?

( 探讨: 舒适感、安全感、自愿性、快感、保护身体健康)

- 年轻人选择性行为有许多不同的原因, 这会产生什么样的误解和问题?

- 我们发现人们经常不会意识到他们的动机和感受, 或者没有分析他们所处的环境, 有什么方法可以 让我们更加了解发生了什么? 有什么方法可以让我们更了解我们的感觉, 了解我们想要或不想要什 么? ( 探讨: 写日志、和信任的人交流、诚实地思考)

\section{教师参考资料}

\section{“强迫选择”之理由：}

1. 男孩们时刻都必须表现出他们想要做爱, 即使他们并不想这样, 我觉得他们这样不好。

2. 如果一个女孩爱他的男朋友, 她就应该通过和他发生性行为表现出来。

3. 我认为大多数青少年对于性有着矛盾的想法: 他们在同一时间内想要却又不想要。

4. 我认为为了发生性行为而给人钱或礼物的行为是可以的。

5. 我认为假使你需要钱, 那么为性行为收钱是可以的。 
6. 我认为一个真正的男人应敢于冒险, 且在性方面有进攻性。

7. 电视或者是杂志上的图像使年轻人觉得他们应该发生性行为。

8. 违背他或她的意愿而强迫其发生性行为，即使你没有运用暴力，差不多就是强奸。

9. 一些女孩表现出只是追求性行为的快感而没有任何情感, 但是她们内心并不想这样, 她们需要情 感交流。

10. 我认识的许多女孩发生性行为都是因为她们觉得必须该做这件事了。

11. 被同性吸引的人群应该比他们同年龄段的异性恋者更晚发生性行为（等到年龄再大些）。

12. 很多年轻人只是不想发生性行为, 他们的想法与艾滋病、怀孕或者成年人告诉他们的东西无关, 他 们只是不想发生性行为, 即使他们有男朋友或女朋友。

13. 性交对于双方来说总是非常亲密和私密的经历。

14. 很多决定发生性行为的人之后都后悔。

15. 很多决定不发生性行为的人之后都后悔。

16. 大部分青少年在发生性行为之前, 都会和他们的伴侣深入地讨论双方是否都想要且乐意发生性关

系, 还会讨论采取保护以免感染和怀孕。 
时间：45分钟

材料：黑板、粉笔; 所选案例的复印本; 一条长绳或结实的长线; 纸夹 (或者胶带)、马克笔

准备工作：按照题目阐释的要求修改好分发资料（详见：“性选择和性强迫的案例”），并将其复印 四份

概述: 学生们针对强迫性行为、自愿性行为和渴望性行为之间的反应程度存在差异的一条连线做 案例研究, 然后讨论说不的权利。( 注: 学生们应该在开始活动前就对性暴力和性同意有 一些了解。)

目的: 使学生描述, 性选择之间的关联, 能够指出什么时候发生性行为虽是自愿的, 但又是不想 要的。清楚了解对性说不的权利, 增强抽象思维能力
活动24

界限在哪 ? 性选 择和性强迫之间

的连接线

1. 在房间内笔直地粘一条线，在一头标注：“完全被强迫，不想要”然后在另一头标注：“完全自 愿，想要"

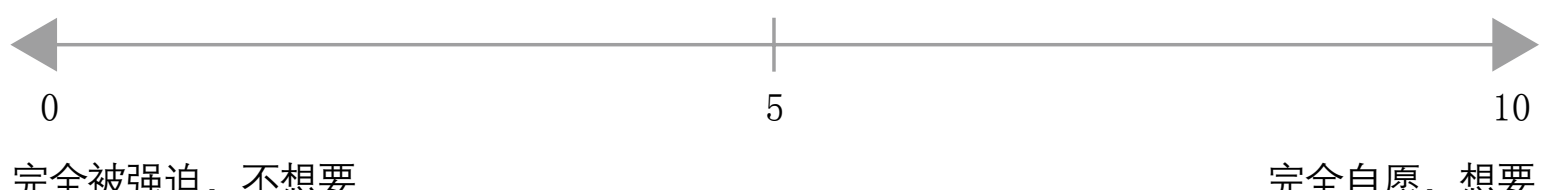

完全被强迫, 不想要

完全自愿, 想要 


\section{2. 介绍这个话题然后解释 : 在被强迫、完全不想要的性和完全自愿，想要的性之间有一个连线。 然后提问：}

- 我们称强迫的性叫什么? 在他们回答后, 在这个连续过程后面加上“强奸”这个词;

如果一个人同意发生性行为, 这是否就是意味着他或她实际上想要发生? 在他们回答后, 提 问：什么时候性行为是自愿的但不是想要的? 这种情况应该在这条线的某一端还是在两端之间 的某个部分?

3. 把学生分成四组, 给每一组分发一份资料和一些纸夹, 并解释:

- 每个组阅读所给的这些情节, 讨论并决定它们应该在线的什么位置;

- 在这个案例上标注你们的名字 (用彩色马克笔标注), 并用纸夹把它们夹在线上合适的的位置;

4. 教师在各组之间走动并提供帮助。即使没有完成所有的案例分析也允许他们花费额外时间去讨论各 自的观点

5. 15 分钟后，从一个组里选一个人让他念第一个案例，解释为什么他们组要把它放在线的某一位 置，给其他组2一3分钟，让他们表明他们所放的位置; 鼓励他们讨论不同观点。注意对其他案例 重复这一步骤

6. 预留10分钟讨论下列问题：

- 强迫别人发生他或她不想要的性行为是对其人权的暴力侵犯。请同学们对年轻人中强迫性行为是否 普遍举手表态;

- 我们讨论过, 一个不想发生也没有被迫发生性行为的人, 仍有可能自愿地和别人发生性行为。谁认 为在年轻人中这是相当普遍的现象? 谁认为这并不常见? (注意记下男孩的回答是否和女孩的回答 相似 ) 
- 在既非强迫而又并不渴望发生的性行为中, 男孩和女孩发生的可能性相同么? ( 探讨: 在性关系中 男孩和女孩是否有同样的力量? 成年的男人和女人呢? ) (注释: 有些情况即使没有被标注到极端 强迫, 也是不能接受的。)

- 一个人是否总能知道他/她的伴侣是否真的想要发生性关系? 有什么方法可以确定呢? ( 探讨: 去 问一个人! 最好预先讨论一下。假如你问了, 但你的伴侣不确定他或她是否想要, 那怎么办? )

\section{教师参考资料 \\ 性选择与性强迫的案例研究:}

给教师的步骤: 在下列案例中选择六到八个（或者写下你们自己的案例）。确保你最后的选择中至少 有一个案例是关于男孩觉得有压力去发生性行为的。修改这些案例, 以使它们更适合你的学生, 并且 有意义。

穆罕穆得和阿玛: 穆罕穆得今晚想要做爱, 但是他的妻子阿玛并不想。她被教导说除非她病了或者正 在月经期, 否则无论什么时候, 只要丈夫想要她就应该和他做爱, 这是一个妻子的责任。所以她就和 穆罕穆得做爱了。 
妮娜和卡尔: 妮娜, 22岁, 和卡尔相处六个月了。卡尔多次告诉她想和她发生关系, 但必须她也想 要。妮娜不确定她的想法, 但是她觉得她应该做她男朋友想要做的事。她知道其他一些年轻妇女都跟 男朋友发生了关系，她担心如果她不同意的话，他可能会离她而去。尽管卡尔从来没有威胁他这么 做。他们在下一次亲密时做爱了。

乔布斯和格蕾丝: 乔布斯和他的女友格蕾丝有一次单独在他的父母家。乔布斯喝醉了, 言语不清躺在 地板上, 格蕾丝也有点醉了, 躺在他旁边。她觉得不受拘束, 自愿地给他口交, 乔布斯觉得很疑惑, 也不确定该说什么。第二天, 他就全忘了。

亨利和玛丽安: 亨利和玛丽安正在热烈地接吻, 在亨利 开始脱玛丽安的衣服时, 她试图阻止他, 并且 说 “不”。亨利觉得她想要更多却又不敢承认, 所以他继续尝试。这样持续五分钟后, 她最终停下挣 扎，只是躺在那，亨利就和她发生了性交。

安捷特和安吉拉: 安捷特和安吉拉只见过几次, 而都是在有人监护的情况下。安捷特看起来是个好 人, 所以当她父母告诉她安捷特想和她结婚的时候, 安吉拉同意了。安吉拉被灌输了任何关于性的事 都是可耻的的观念, 她听说第一次会疼而且会使她流血, 她非常害怕。她几乎不了解安捷特, 一想到 他碰触她的身体, 她觉得差愧。她对做爱没有兴趣, 也不觉得兴奋。但是她知道结婚后就必须在结婚 之夜发生性关系，她就让安捷特跟她做爱了。

西拉和詹姆斯: 西拉最近一次拒绝她的丈夫詹姆斯, 他威胁她并殴打了她, 把她的一双眼睛都打肿 了。今晚她不想和他做爱，但是他可能会再打她，所以她没有拒绝。

林和杨: 林的男朋友杨老是催逼她跟他发生性关系。她知道自己并非真的想做, 但是她和杨独自去了 隐蔽的地方，她觉得这是必然的，于是就让杨做了他想做的，林没说一句话。

里奥和海伦娜: 里奥和海伦娜是大学同学, 他们在一起约会, 有几个月了, 被对方所吸引。他们没有 很深地相爱，但是都同意发生关系。在协商用安全套后，他们发生了性交。 
吉娜和艾哈迈德: 吉娜来自一个贫困家庭, 在艾哈迈德的商店工作。她的薪水是家庭的主要收入。一 天艾哈迈德开始摸她, 并且在关店后把她拉到后面的储藏室, 掀起了她的裙子。吉娜很慌乱, 害怕丢 掉工作。她开始反抗，但是艾哈迈德说: “你难道不想在这干了? ”，她就屈服了。

茱丽亚和奥斯卡: 奥斯卡不确定他是否真的想和茱丽亚做爱, 但是茱丽亚提出了, 他担心万一朋友知 道他拒绝和荣丽亚作爱的话会耻笑他, 于是他就跟荣丽亚做爱了。

彼得和劳拉: 彼得总是给劳拉买礼物和其他她需要的东西。今晚他们外出吃饭, 彼得结账。尽管劳拉 没有准备好做爱，但是她觉得她欠了彼得的，所以没有拒绝他。

薇拉和威廉姆: 薇拉和威廉姆做过一次爱了。今夜威廉姆告诉薇拉他想要她都想了一天了。薇拉只想 和他出去转转, 聊聊天。但是她认为, 既然他们做过一次了, 就没有回头路了。当威廉姆开始脱她的 衣服时, 薇拉什么都没说, 他们就再次做爱了。

艾玛和罗伯特: 一天晚上, 艾玛喝多了。罗伯特发现她喝得烂醉, 身尚在床上。他脱掉她的衣服和她做 爱了, 她并没有醒来。 


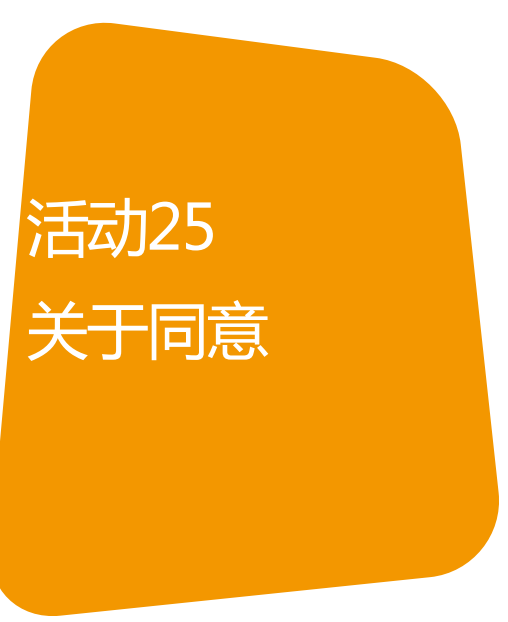

时间：步骤1一 6 : 45分钟 步骤7-8: 45分钟

材料：黑板、粉笔; 精选案例; 教师参考资料; 答案解释

准备工作：选择4-6个反映共同情况的案例, 将他们的姓名和有关细节进行适当改变, 或者编制一些 其他案例。用一些能够给出自由、知情、同意的人物案例, 和一些他们不能这样做的案 例。如果需要的话, 可以修订教师讲稿。事前在黑板上写好指导原则, 找出你们国家有关 允许发生性行为年龄限制的规定

概述：学生学习同意性行为的概念。他们进行实例研究, 观看短剧并加以讨论

目的：帮助学生明白并理解对于任何性行为来说同意的重要性; 增强抽象思维能力

\section{1. 用下列的解释和问题介绍这个活动}

- 不管是在家里、学校里或是在工作中, 我们所有人都有责任。但有时候我们被同龄人或成人要求 或告知去做我们责任外的事情, 这是令人迷惑或者是令人烦恼的。

选择：根据如下的步骤来更具体的介绍这个概念：

同意做一件事是什么意思?（探讨：同意，表明或者是表达一个意愿）

我们为了不同的原因同意去做某些事。有时, 我们同意是因为想做。举个例子, 如果一个朋友请 你去踢足球, 你也想去踢。或者有一些我们并非真正想去做的事情, 但是因为其他原因它对我们 重要, 我们也可能同意了。举个例子, 当你的老师给你布置作业时, 有可能你不想做但还是做 了, 因为你想取得好成绩。但是, 也有时我们做一些我们不想做的事情是因为我们很难说不, 或 
是我们不想表现得粗鲁。这也许涉及到生活中的一些小事, 就像有人募了东西给你, 虽然你不喜 欢, 但是你还得吃。也许又涉及到特别重要、能产生较大影响的事情。谁能举出几个关于年轻人需 要作出重大决定的例子? ( 如果学生们没有提到性, 可以将其添上 )

- 你是否曾经同意做一些不是你的责任而且你又不想做的事, 有此经历的请举手。谁能跟我们分享一 下?

- 一个人同意去做他并不想做的事情有哪些原因呢? 在黑板上写下回答;

- 今天我们将会着手研究在性行为中同意意味着什么。

2. 提问 (在黑板上写下回答) :

- “同意”这个词是什么意思?

- “自由、知情的同意” 是什么意思?

3. 让学生大声读出在性行为中自由、知情的同意的指导原则

4. 将班级同学分几个小组, 每个组都有不同的案例, 然后解释:

- 学生有10分钟讨论你们的案例, 准备一个 (2到3分钟的) 的短剧, 然后考虑这个案例中哪些符合 自由、知情的同意的原则, 哪些不符合;

- 一名学生将给全班读这个案例, 其他两个人表演, 一到两名学生解释, 案例中哪些符合或者不符合 指导原则。( 给出10到20分钟解释这个任务, 让各组准备工作。教师在组间来回走, 以便需要时 给予帮助 )

5. 让一组展示a ) 他们的案例 b ) 他们的短剧； c ) 关于案例中的人物是否满足 “同意”原则的集体观 点, 小组讨论得出结果

6. 询问一下谁赞成或者不赞成这一组短剧符合 “同意”原则，讨论直到得出一致意见 


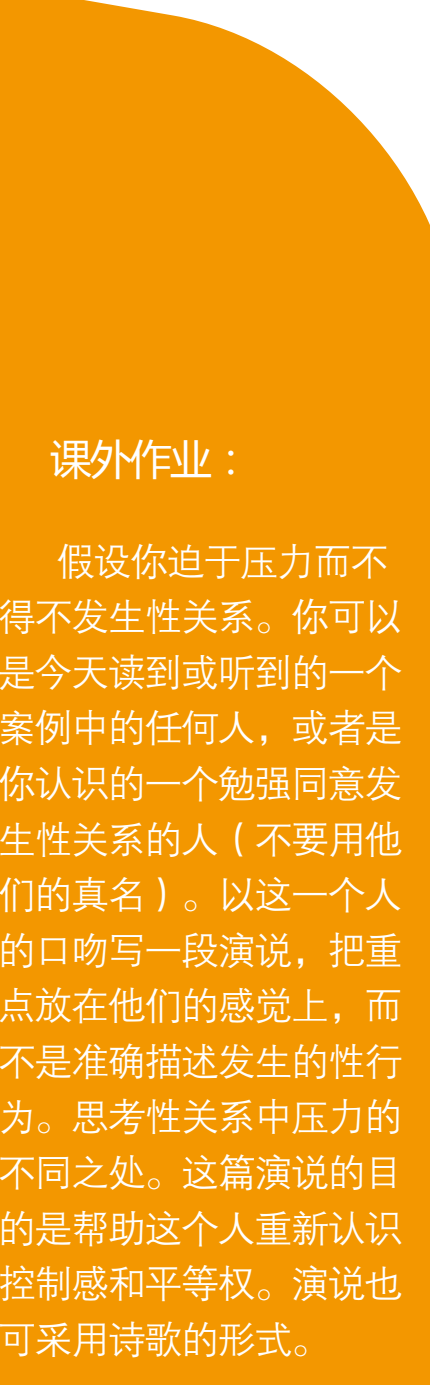

假设你迫于压力而不 得不发生性关系。你可以 是今天读到或听到的一个 案例中的任何人，或者是 你认识的一个勉强同意发 生性关系的人 (不要用他 们的真名）。以这一个人 的口吻写一段演说，把重 点放在他们的感觉上, 而 不是准确描述发生的性行 为。思考性关系中压力的 这篇演说的目 控制感和平等权。演说也 可采用诗歌的形式。 
- 你要尽可能避免为物质和钱财而被迫发生你不想发生的性行为。

\section{学生阅读材料 :}

案例研究 : 这个人能给出明确有效的同意吗?

A

安娜现在19岁, 她的男朋友艾现在22岁, 他们相识并约会有6个月了, 开始做爱也有一个月了。安娜一 般情况下很享受性行为, 但是某些时候她却不太想发生性行为。在那种情况下, 艾经常说, “如果你 真的爱我, 你就会想要” 或者说 “如果你不跟我做爱, 我就会找其他人”。她不想失去他, 所以经常 妥协。安娜能否自由地做出同意的决定呢?

B

本尼知道自己感染了艾滋病病毒, 但是他却没有告诉任何人, 他开始跟碧约会。最近他们讨论到做爱 这件事。本尼打算使用安全套而不告诉碧他感染了艾滋病病毒。在这种情况下, 碧能自由、知情的做 出同意与否的决定吗?

C 卡洛和可莉都20岁了, 他们交往了一年, 也互相坦诚过去的情史, 他们愿意和对方做爱。他们对做爱 这件事请进行了讨论, 同意去一个诊所获取有关信息, 然后就可以决定如何能做到安全且避免意外的 发生。在这种情况下可莉能否做出自由、知情的同意吗?

D

当和迪娜18岁了, 他们决定做爱。迪娜说她害怕怀孕, 但是当肯定地说, 你第一次做爱的时候是不可 
能怀孕的。在这种情况下, 迪娜能否做出自由、知情的同意?

E

伊娃14岁, 她就读一所中学, 伊多18岁, 他与伊娃的爸爸一起工作。伊多拜访伊娃家的时候与伊娃 相识。伊多和伊娃开始在远离她家的地方约会, 有时候伊多会在伊娃需要的时候给她礼物和钱。最近 他开始告诉她他有多爱她, 而且他真的很想和她做爱。在这种情况下, 伊娃能做出自由、知情的同意 吗?

F

弗林18岁了, 他想和他的女朋友一17岁法蒂玛做爱, 他们坦诚双方都从未有过性经历。他决定读他 的医生妈妈的一本有关成长、性、计划生育和性传染疾病的书。他问他的女友的想法, 并把那本书借 给了她。她读了这本书后, 他们又对这一话题进行讨论。她说想再等等, 所以弗林同意了。在这种情 况下, 法蒂玛能做出自由、知情的同意吗?

G

24岁的吉娜, 在一个学生家庭聚会中喝多了。一个曾经和她发生性关系的伴侣, 把她推进卧房, 开始 脱掉她的衣服, 她的意识若有若无。在这种情况下, 她能做出自由、知情的同意吗?

$\mathrm{H}$

海林的母亲教导她说, 如果不想失去她的丈夫, 女人就该做任何他要求她做的事情, 这一点对女人很 重要。她与胡果订婚了。他说既然他们已经订婚了, 就该做爱了。海林坚信她应该再等, 而且她想在 结婚之前时保留处女之身。但她想, “他将会成为我的丈夫, 而且我妈妈说我应该服从他。” 在这种 情况下, 海林能做出自由、知情的同意吗? 
伊萨克曾经有过几个交往对象, 但他却让新男朋友伊凡相信他仍是处男, 实际上伊凡是处男。当他们 做爱的时, 伊凡因为觉得没有传染的风险所以同意不用安全套。在这种情况下, 伊凡能做出自由、知 情的的同意吗?

J

金20岁, 约瑟夫19岁了。六个月前金问她的男朋友约瑟夫是否想和她做爱。他们都能相互开诚布公, 所以他告诉她他害怕后果, 因此他并没有准备好。他说他现在只想亲吻和抚摸对方。金非常想要尝试

一下做爱, 但是她还是同意了约瑟夫。现在约瑟夫认为他已经准备好了, 他问金在哪能获得更多的信 息以避免任何的危险。在这种情况下, 约瑟夫能做出自由、知情的同意吗?

教师参考答案 : 案例研究的参考答案

“案例研究 : 这个人能给出有意义的同意吗?"

注释: 你必须知道你们国家的法律对同意进行性行为的最小年龄的规定, 以便评定在不同情景中的人 是否已达到可以进行性行为的年龄。你可能需要根据具体情况对情景和答案进行改动。

$A$ 不。当安娜说她不想做爱时, 艾不尊重安娜的决定。她也不确信自己有权决定自己的事情, 因而在爱 侣关系中缺乏自主力。

$B$ 不。B碧对性伴侣的健康状况没有准确的信息。

$\mathrm{C}$ 是。他们两人都为自己做出了决定且达成一致; 他们做决定时都有着清晰的头脑; 他们不受物质和 
金钱上的动机所影响; 他们能够对自己的决定进行交流, 知道他们的决定包含着什么, 也知道他们采 取保护措施；他们对他们的过去进行了诚实的交谈; 他们达到同意性行为的法定年龄。

D 不。迪娜不知道危险所在, 也不知道如何保护自己。

$E$ 不。14岁小孩的性同意一般不具有意义, 因为他们还不够成熟。为了进一步关注成熟以及儿童权 利, 法律对同意发生性行为的最小年龄进行了规定, 并且视童婚为非法。

$\mathrm{F}$ 是。法蒂玛认为她有决定做爱的权利, 她的伴侣尊重她的决定。她做决定时头脑清晰; 没有经济或物 质动机的压力。能够对她的决定进行交流以及实施, 知道它包含了什么, 它的危险所在, 如何去保护 她自己。她对他伴侣之前的性行为有着清楚的了解。在大多数国家, 她被认为已达到年龄, 如果她愿 意的话, 可以去做爱。但请核对一下你所在国家的法律条文。

$G$ 不。吉娜謁醉了并且几乎没有任何意识, 她无法为自己做决定, 并且她的伴侣并没有与她交流。

$\mathrm{H}$ 不。海林知道她想要什么, 但是她似乎并不确信自己有自行决定的权利, 或者说她对自己的生活缺 乏足够的自主控制力。

| 不。伊凡对他的伴侣之前的性行为并没有准确的了解。

$\mathrm{J}$ 是。约瑟夫相信他有为自己做决定的权利, 他能够进行交流并实施自己的决定。同时他的伴侣也尊重 他。他头脑清醒, 没有过度的经济和物质方面动机。他知道性行为的危险和保护措施。他已经达到了 作出同意性行为的法定年龄。虽然我们不知道这对伴侣是否就之前的性行为以及毒品使用的问题进行 过讨论，但是我们知道他们相互都十分开诚布公。 


\section{时间：45分钟}

材料：黑板、粉笔，有关性政策话题的头条新闻

准备工作：如果需要的话, 可以删去任一头条新闻。把头条新闻抄至黑板

概述：学生选择一条反映性规范变化相关的头条新闻, 写下个人的回答

目的: 帮助学生明白性规范是随着时间变化着的。弄清他们对所选话题的个人价值观，增强批判 性思维能力和写作技巧

\section{活动26}

热点政策话题与

个人回答

\section{1. 通过解释以下几点介绍这次活动：}

- 不同社会中关于性的态度也不同, 并且这些社会态度也在不断变化中。今天, 我们要找出并思考这 些态度是如何变化的;

- 阅读黑板上的头条新闻, 这些新闻大部分是由真实的事件改编而成。选择一条新闻, 并写一页纸左 右的个人观点。学生可以写下自己对这个话题的任何感觉和想法。但是试着深入思考, 并以你对 这个问题的看法在10至15年内会发生什么变化来结束回答。花5分钟来看题目单子，15分钟完成写 作。之后一些人要大声读出他们的回答。

2. 对于每个头条新闻，让一个志愿者来读他或她答题的内容，读完后，谢谢这个学生。如果需要的

话，阐述这个问题是什么，并纠正明显的错误。根据以下提示鼓励讨论：

- 这条新闻让你感觉如何; 
- 你对此有何回应;

- 这个问题是否与我们国家相关;

- 有没有其他意见。

( 每条头条新闻准备2到3分钟, 有些头条新闻可以不选, 这样可以有更多时间去讨论那些选中 的新闻 )

3. 结束练习时，要再一次提醒学生规范总是在不断变化的，提问：

- 规范是否总是向更加灵活的方向变化? 或者规范是否可能向更严格或者更有限制性的方向变化?

- 有关性的或者其他问题的社会规范会自己变化吗? 什么, 或者是谁, 导致了性规范的变化? ( 探 讨: 广泛的社会现象（例如高学历和传媒全球化）和个人角色（公众领袖和社区成员）)

- 人们通过角色扮演、教育他人、提倡政策的改变带来这些变化。

\section{教师参考资料}

有关性政策热点的“头条新闻”

- 女性控告性骚扰获得胜诉: 此类案件的第一次胜诉;

- 青少年诊所开张: 本州第一所;

- 本地男性举行一次反对性暴力的游行: 宣称真男人不强奸; 
- 允许同性恋者结婚的法律正式生效: 第一对新人在早上八点结婚;

- 14岁女童在强迫婚姻中逃婚;

- 女孩们抱怨要求她们老是衣服裹身的穿衣守则;

- 60 位年轻男性签名保证他们只会跟没有行割礼的女性结婚;

- 警察逮捕了一位宣称只要能跟五位处女做爱就能治愈艾滋病毒的携带者;

- 一项新研究显示仍有父母将自己的女儿卖给性商人;

- 总统说他在知道他自己的女儿是一位女同性恋之后对于同性恋者的态度有所改变;

- 流产法变得更加严格, 提供流产手术的人会被逮捕。 
人亚新关系 


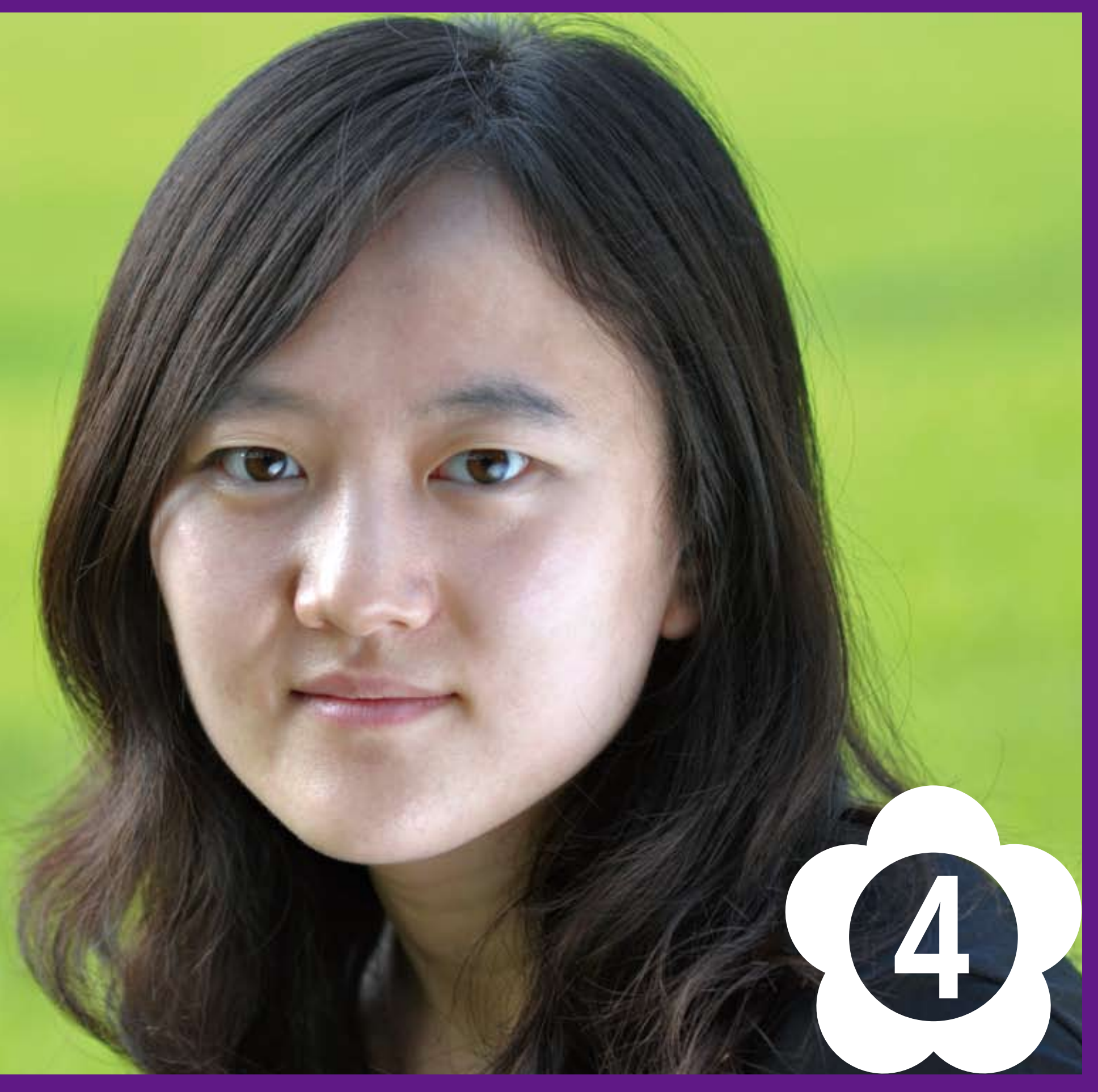


时间：45分钟

材料：黑板、粉笔

准备工作：只须阅读步骤

概述：学生们将他们的人际关系进行视觉上的展示

目的：帮助学生识别出生活中不同的人际关系, 并且说出他们所珍视的自己拥有的品质, 以及他 们希望从亲近的人际关系中获得怎样的品质。强化学生的批判性思维能力

1. 向学生解释，他们将对各种人际关系进行辨别。让学生将自己与他人的关系列个表。

2. 请一些学生自愿来与大家分享他们所写下的内容。在黑板上写下他们所提到的多种关系，比如，亲 戚关系、朋友关系或邻居关系

3. 在黑板上画出一个像下图那样的，由四个同心圆组成的图表

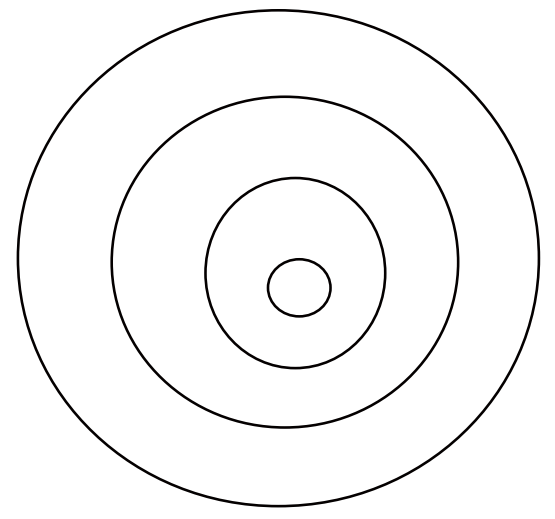


4. 让学生们拿出一张空白的纸，向学生解释道 :

- 画出四个相套的圆圈, 从大到小, 就像我刚刚在黑板上画出的那样。让最大的圆圈占满整张纸、

- 在最小的圆圈里写下你的名字 (或画下你的自画像)、

- 想一想你生活中不同的人。在小圈外面写下那些与你最亲近的人的名字 (或画出他们简单的形 象 )、

- 在另外两个外面的圆圈里, 写下与你不是那么亲近的人的名字 (或简单画出他们的简单形象)。

5. 让学生们与临近的同学组成三或四人的小组，每个人用约 2 分钟向小组中的其他成员解释自己的所写 或所画

6. 让学生们展开如下的讨论：

- 你小组中的成员列出的各种关系相同吗? 不同的话, 区别在哪儿? 同学们将家庭成员, 朋友, 邻 居，教师，宗教领袖等人分别放在相同的还是不同的圆圈里?

- 说出一些词语, 它们可以描述在你最亲近的人的身上, 你所重视的品质。( 探讨: 诚实、有礼 貌、乐于分享、关心他人、值得信任、逗人、理解感、可靠、有趣、充满爱意。将这些写在黑板 上。)

7. 留出10分钟时间让学生思索他们在人际关系中重视的品质：

- 在你的图表中想出一个你愿意将他移近核心圈的人。用一个最恰当的词来形容你看中的是他哪 一方面、

- 现在从黑板上挑出一两个你认为别人会用来形容你的词、

- 用装饰字或特别的风格写出你重视的品质 (你自己或别人的), 来表示对它的赞赏、

- 在你写写画画的时候, 想一想这一品质对你意味着什么。 
时间：45分钟

材料：黑板、粉笔，每一小组一份案例研究材料

准备工作：阅读案例研究材料, 进行必要补充或替换, 以确保它们能够反映当地社会中常见的不平等 现象。将每个案例研究复印在单独的一张纸上

概述: 学生阅读关于人际关系中不平等现象的案例研究材料, 找出不平等现象并讨论其对人际关 系影响

目的：帮助学生了解到社会不平等现象是如何影响到亲密关系的; 增强分析思考能力

1. 向学生们解释 :

- 今天我们将讨论不平等现象, 以及人与人之间权力不同会如何影响我们的人际关系。在我们社会 的不同群体中, 不平等现象是以何种方式存在的? ( 探讨: 种族、社会阶层; 建立在性别、年 龄、阶级、民族、性取向、身体能力等多方面的不平等现象。）

- 学生将分成四个小组, 每个小组一同阅读一份案例研究, 并讨论。然后回答问题。

2. 给每组发一份案例研究和一份供讨论问题，给他们10分钟时间

3. 给其中一个小组 5-7分钟时间，朗读他们的案例研究，并说出他们对于所讨论的问题的回答。然后留 出几分钟让其他学生进行评论

4. 对其他三个案例研究也轮流进行这一过程

5. 最后，鼓励学生们说出社会上的一种不平等现象是怎样反映到自己的人际关系中的，并探讨这些不 平等在他们身上造成的后果 
案例研究材料 :

第一组 :

我之所以被我的男朋友吸引, 是因为他较年长, 而且没人认识他。他显得神秘而令人兴奋。我们坠入 了爱河, 如今形影不离。你知道那感觉的。我真的非常喜欢听他讲他的各种经历。当我们一起出去 时, 大多情况他决定去哪, 因为从来都是他付账, 而且他对于哪里好玩也十分了解。他对我影响特别 大。我从来对体育不感兴趣，但是他喜欢橄榄球，所以我现在大量的空闲时间是在和他一起在球场或 电视上看比赛。

在我们认识对方几个月后, 他说他想和我做爱。我很犹豫, 但是我想到如果我不同意的话他有可能离 开我。他毕竟今年23岁, 经历过性事。那些天, 我几乎没见我的朋友们。他们抱怨说我变了。我很想 念他们, 并且有时我会想起, 我曾经在学习上花费更多的时间, 还有我是多么热爱阅读。但是, 你知 道, 我认为值入爱河后就是那样。

问题 :

1. 不同社会群体中存在着各种不同的不平等现象（例如，人们可能会由于他们的民族、种族、经济 阶层、性别、年龄、或其他因素而拥有更高或低的地位）。案例里的这段关系中存在着怎样的不平 等现象?

2. 由于他或她社会地位的不同，在这段关系中谁占有更为强势的地位? 这种不平等现象的基础是什 么? 这种强势地位是如何表现出来的?

3. 由于他或她的社会地位的不同, 谁在这段关系中处于弱势地位? 这种弱势地位是如何表现出来的?

4. 这种不平等现象给双方带来 (如果有影响的话) 哪些影响? 它又是怎样影响他们的关系的?

5. 你认为案例中这种现象在我们的周围存在吗? 对这些现象发表评论。

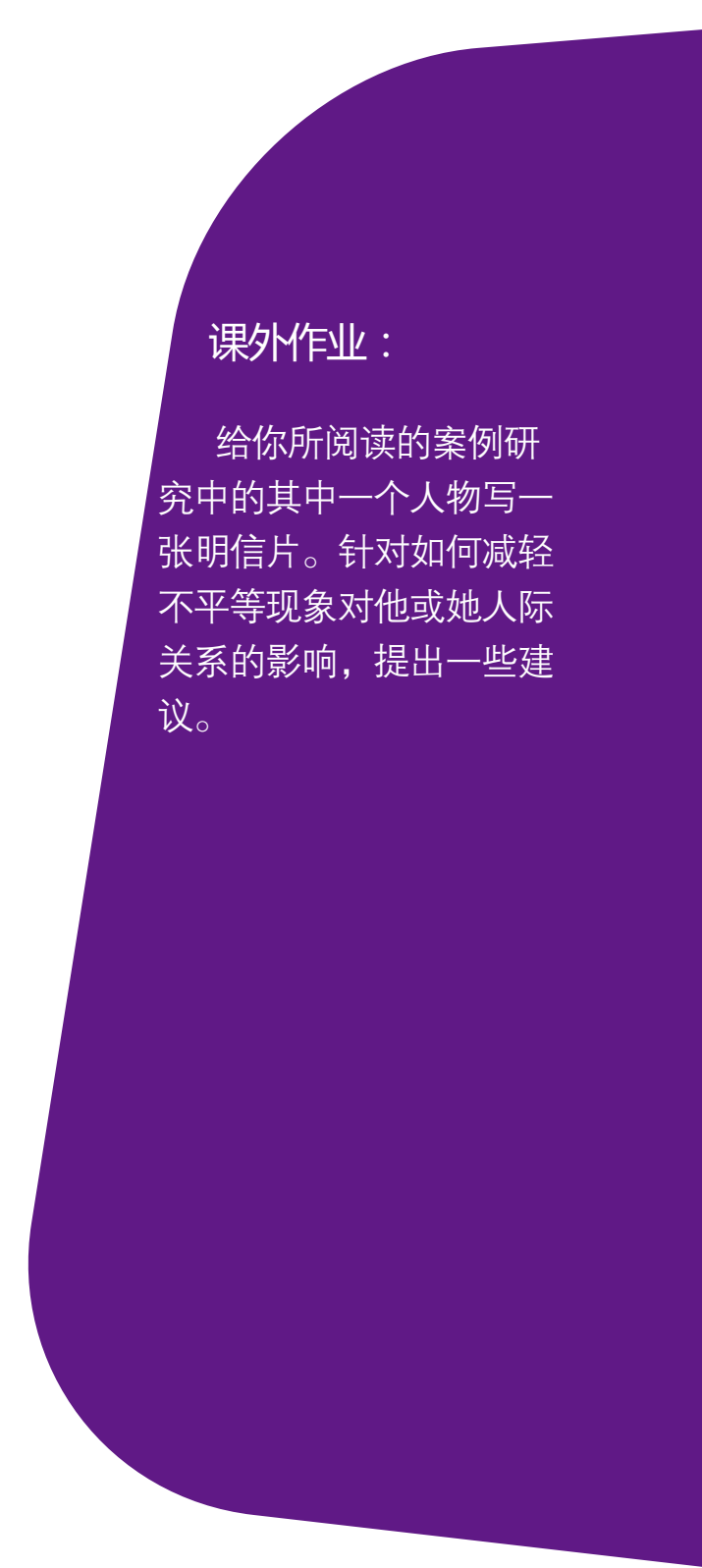


第二组 :

去年我开始和一个橄榄球队的男孩约会, 我们之间的关系开始变得更加亲密。有一天在村礼堂举行的 一个聚会上, 他暗中向示意我在礼堂后面和他见面。我父母当时也在, 但是我知道他们会以为我是去 找其他女孩了。所以我和他悄悄溜了出来, 一同在礼堂后面的树丛间散步。当我们走进树丛的时候他 开始吻我, 并抚摸我的胸部。我知道我们是要做爱了。我其实并不想要。我只是想和他聊聊天, 接接 吻, 没别的了, 但是他想要, 而我一直顺着他, 结果我们发生了性关系。

我后来哭了, 因为我明白我这样做是对我父母的不尊敬, 而且我失去了自己的童贞。我知道安全套可 以防止一个女孩怀孕或染病, 但是当时我在想其他事情, 包括我的父母知道这件事后会怎么想。我更 为自己的失贞担忧。当我真的怀孕时，我的父母勃然大怒，禁止我再见我的男友。

问题 :

1. 不同社会群体中存在着各种不同的不平等现象 (例如, 人们可能会由于他们的民族、种族、经济阶 层、性别、年龄、或其他因素而拥有更高或低的地位 )。案例里的这段关系中存在着怎样的不平等现 象?

2. 由于他或她社会地位的不同, 在这段关系中谁占有更为强势的地位? 这种不平等现象的基础是什 么? 这种强势的地位是如何表现出来的?

3. 由于他或她的社会地位的不同, 谁在这段关系中处于弱势地位? 这种弱势地位是如何表现出来的?

4. 这种不平等现象给双方带来（如果有影响的话）哪些影响? 它又是怎样影响他们的关系的?

5. 你认为案例中这种现象在我们的周围存在吗? 对这些现象发表评论。 
第三组 :

当我三十五岁的时候, 我觉得自己得结婚了。我终于有了一份工作, 能够负担一个家庭了。我大学毕 业时失业的人很多, 我在家乡很难找到工作。最终我选择到异地寻找工作机会。当我回家看望母亲的 时候, 我决定在村子里给自己找个妻子。我陆续见了几个年轻女人, 对其中一个很有好感。她今年 二十岁, 是个文静的人, 虽然读完了小学, 但从没真正从村子走出过多远。尽管我跟她说, 在能够把 她接到我身边之前, 她只好先和我母亲一起生活, 她还是答应与我结婚了。

我们有了第一个孩子后, 我不希望她再吃避孕药了, 所以我采取体外射精的方式。我尽自己最大的努 力为她和孩子提供一切。她还和我母亲生活在一起, 从没惹出过什么乱子, 也没有任何要求。我爱 她，但是有些事我不能跟她谈起，因为她对这个世界了解很少，所以很多东西无法理解。

问题 :

1. 不同社会群体中存在着各种不同的不平等现象（例如, 人们可能会由于他们的民族、种族、经济 阶层、性别、年龄、或其他因素而拥有更高或低的地位）。案例里的这段关系中存在着怎样的不平 等现象?

2. 由于他或她社会地位的不同, 在这段关系中谁占有更为强势的地位? 这种不平等现象的基础是什 么? 这种强势的地位是如何表现出来的?

3. 由于他或她的社会地位的不同, 谁在这段关系中处于弱势地位? 这种弱势地位是如何表现出来的?

4. 这种不平等现象给双方带来 (如果有影响的话) 哪些影响? 它又是怎样影响他们的关系的?

5. 你认为案例中这种现象在我们的周围存在吗? 对这些现象发表评论。 


\section{第四组 :}

我出国工作了一段时间, 结果和一位同事恋爱了。当我怀孕了的时候, 我们决定结婚。我的父母不同 意这桩婚事, 原因是他的家境贫困。他们说, “你不知道和一个与你不相称的人结婚的后果。你回家 把孩子生下来? 我们会帮助你的。” 但我们还是结婚了。我们决定回到我的祖国, 这样他就可以在我 工作的时候, 取得一个大学文凭。但是在他开始学业之前, 他得先过语言关。

不幸的是, 他发现这很难。他在家照看孩子, 尝试着学习, 但是感到十分孤单, 变得越来越沮丧而且 变得很自卑。他不得不依靠着我的收入生活, 同时对我们定期给他家里寄钱感到愧疚。我不喜欢他不 学习、不找工作或不工作的状态, 但是我能控制自己不说什么。这是个如此敏感的话题, 我不希望伤 害他的感情。最终他交到了一些同乡的男性朋友, 经常和他们一起打发时间, 泡酒吧、喝酒。有一次 他甚至对我动粗，这真的给我们的生活敲响了警钟。

问题 :

1. 不同社会群体中存在着各种不同的不平等现象（例如, 人们可能会由于他们的民族、种族、经济 阶层、性别、年龄、或其他因素而拥有更高或低的地位 )。案例里的这段关系中存在着怎样的不平 等现象?

2. 由于他或她社会地位的不同，在这段关系中谁占有更为强势的地位? 这种不平等现象的基础是什 么? 这种强势的地位是如何表现出来的?

3. 由于他或她的社会地位的不同，谁在这段关系中处于弱势地位? 这种弱势地位是如何表现出来的?

4. 这种不平等现象给双方带来（如果有影响的话）哪些影响? 它又是怎样影响他们的关系的?

5. 你认为案例中这种现象在我们的周围存在吗? 对这些现象发表评论。 
时间：45分钟

材料：黑板、粉笔、写有词组的纸张

准备工作：根据学生的文化、社会、语言灵活调整课程。取出五张纸, 将以下短语分别写在五张纸 上: 迷恋、恋爱中、浪漫、性吸引力、姤妒

概述：让学生们将爱与其它相关情感区分开来

目的：使学生能够区分爱、迷恋和矮妒; 增强分析思维能力

\section{活动29}

这是爱吗?

1. 将学生分为五组，让每组从准备好的纸张中抽取一张。在黑板上写，“爱与 的区别是什么?"

2. 让各组取出一张纸，将黑板上的句子抄在纸上，把他们纸条上的词添在空里。让他们讨论各自的问 题，并写下他们的回答。

3. 大概五分钟后，请一组同学朗读他们的问题和回答。可以向大家提出下面这些问题，来开展一个简 短的讨论 :

- 有人不同意他们的回答, 或想对他们的回答进行评论吗?

- 文化环境是否给予女孩与男孩同等权利来经历这种感情?

- 对于有些人将爱与这种感情相混淆的情况, 你能举出例子吗? 当人们将这种感情与爱相混淆的时 候, 会发生什么状况?

- 这种感情会是爱的一部分吗? 
4. 其余四组重复第3步的过程

5. 留出10分钟来讨论以下问题：

- 年轻人是如何获得对爱和浪漫的认识?

- 电影和言情小说对于这些情感的描绘符合现实吗? 如果不符合的话, 你认为它们给年轻人（对爱 情的预期）造成了怎样的影响?

- 为何理清你自己对于这些感情与爱的区别至关重要? 
时间：45分钟

材料：黑板、粉笔

准备工作 : 汶览工作表中列出的情况, 确保你能够解答学生提出的任何相关问题

概述: 学生仔细考虑, 年轻人通过沟通, 实现一段安全而舒适的性关系需要什么条件, 包括成熟 程度、自我意识、自制力、交流能力。( 注释: 在开始这一节课前, 你的学生应当已经了 解性行为和性强迫。)

目的: 帮助学生明确通过沟通, 实现一段安全而舒适的性关系需要的能力; 增强学生的批判性思 维能力

\section{步骤}

1. 通过以下这些内容来介绍活动：

- 年轻人对于他们是否和何时发生性行为有不同的决定标准。有些人等到一定年龄; 另一些人等到他 们订婚或结婚, 还有些人之所以发生性行为是另外一些人或多或少地替他们做了主。权衡是否进行 性行为这个决定时, 很多年轻人考虑的是对双方关系的感受;

- 决定是否发生性行为对于很多年轻人来说是困难的。这个活动会帮助你思考成熟度、自我意识以及 交流技巧在决定你是否准备好发生性行为中所起的作用。这个活动还能帮助明确什么对你最重要。

2. 让同学们两人一组，给每一组一张工作表 ( 附有操作说明 )。向他们解释 :

- 阅读这份资料上列出的条目。这些感受和条件可能对于开展一段舒适而安全的性关系有重要意义; 如果一个人想与人发生性关系, 而且这些条件中的绝大部分 (或全部) 在他们的关系中都存在, 这 个人更有可能获得舒适而安全的性行为;

- 如果一段关系并不具备这些条件, 这个人更有可能获得不舒适且存在风险的性行为。

\section{活动30}

决定是否发生性

行为 
3. 大声读出工作表的操作说明。确保每个人都理解了活动内容。告诉学生，在回答几个问题时，他 们会挑出不同的条目。他们需要得出一致的回答，但是如果他们不能做到的话，他们可以挑出更 多条目。给他们15分钟讨论问题并记录他们的回答

4. 将大家聚集到一块。回顾工作表上第1至5个问题，并让学生们说出一些他们的回答。问问大家同不 同意同伴的回答

5. 对于工作表中的第6个问题，多花些时间来检查学生们的回答。将他们的回答记录在黑板上。以下这 些问题能够引导讨论 :

- 什么情况下, 男孩似乎掌握着优先权?

- 哪些条件对女孩来说更重要?

- 你怎样解释男孩与女孩之间优先权的不同? 对于这种不同，你的感受是什么?

6. 以对工作表中第7和第8个问题的讨论来结束活动

- 然后提问:

- 这些条件中有哪些对于你来说不重要? 你在决定能进行性行为时, 哪些条件是绝不会考虑的?

不论你在青少年时期就发生性行为, 还是等到成年了或结婚了再进行性行为, 确保满足这些条件 能够使你的性行为更加的舒适和安全。( 如果你打算讲其他内容, 请告知你的学生。例如, 《指 南手册》的第一卷中包括交流、身体和解剖学、性健康/艾滋病病毒、社会性别的内容。）

工作表操作说明 :

我准备好了吗? 我如何决定?

在这个练习中, 一个和你一样大的年轻人正在尝试决定, 是否与他人发生性行为。这个年轻人希望做 出正确的决定, 提出了这样的问题: “在考虑我能准备好进行性行为的时候, 哪两个感受或条件是我 
最应该考虑的? ” 这个人向三个人提出了以上问题, 这三个人是: 最好的朋友、顾问（或心理医生） 和艾滋病患者。

现在按以下步骤进行 :

1. 通读你工作表上的条目。

2. 讨论并得出结论, 这三个人将如何回答年轻人的问题。

3. 最好的朋友: 在你认为最好的朋友会说出的两个感受或条件的格子里, 画一个童真的笑脸。( () )

4. 顾问: 在你认为顾问会说出的两个感受或条件的格子里, 画上一个对勾。（ $（ ）$

5. 艾滋病患者: 在你认为艾滋病患者会说出的两个感受或条件的格子里, 画上一个艾滋病防治标 志。( R)

6. 讨论: 你认为这些感受或条件对于男孩比对于女孩来说更重要吗? 在你认为对男孩来说更重要的条 件边上画一个男孩的标志，在你认为对女孩来说更重要的条件和感受边上画一个女孩的标志。

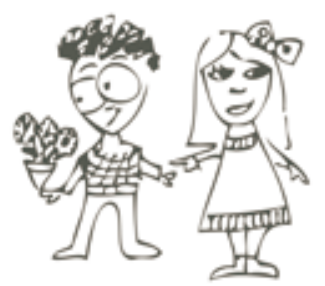

7. 现在来思考当年轻人决定第一次发生性行为时, 哪两种感受或条件是他们经常想不到的, 或者觉得 难以达到的。在这些条目的后面画一个哭脸。( $(\dot{)})$

8. 你认为还有什么感受或条件在第一次发生性行为之前是十分需要考虑的?

学生工作表

准备好发生性行为 


\begin{tabular}{|c|c|c|c|}
\hline $\begin{array}{l}\text { 发生性行为所需具备的 } \\
\text { 感受或条件 }\end{array}$ & 请画标注 & $\begin{array}{l}\text { 发生性行为所需具备 } \\
\text { 的感受或条件 }\end{array}$ & 请画标注 \\
\hline $\begin{array}{l}\text { 感到你尊重自己和伴侣 } \\
\text { 的价值观 }\end{array}$ & & $\begin{array}{l}\text { 感到能够自然的谈论 } \\
\text { 怎样使对方感觉好 }\end{array}$ & \\
\hline 与伴侣感觉亲近 & & $\begin{array}{l}\text { 感到能够自然的和他 } \\
\text { 人谈论安全套的使用 }\end{array}$ & \\
\hline 感到你与伴侣相互尊敬 & & $\begin{array}{l}\text { 有安全套 ( 或其他避 } \\
\text { 孕措施 ), 并且知道 } \\
\text { 如何使用 }\end{array}$ & \\
\hline 感到你与伴侣相互信任 & & $\begin{array}{l}\text { 知道自己能感染了艾 } \\
\text { 滋病病毒 }\end{array}$ & \\
\hline $\begin{array}{l}\text { 感到你和你的伴侣一同 } \\
\text { 决定了此事, 并且你们 } \\
\text { 二人都想进行性行为 }\end{array}$ & & $\begin{array}{l}\text { 感到能够自然的谈论 } \\
\text { 你和你的伴侣能感染 } \\
\text { 了艾滋病 }\end{array}$ & \\
\hline $\begin{array}{l}\text { 对自己的身体感到基本 } \\
\text { 满意 }\end{array}$ & & $\begin{array}{l}\text { 你们两人都感到自己 } \\
\text { 可随时说停止吧 }\end{array}$ & \\
\hline $\begin{array}{l}\text { 对于自己如何获得性满足 } \\
\text { 的理解, 感到很有自信 }\end{array}$ & & 尊重各自的隐私 & \\
\hline $\begin{array}{l}\text { 感到自己对对方有性吸 } \\
\text { 引力 }\end{array}$ & & $\begin{array}{l}\text { 感到自己没有在事后面 } \\
\text { 临嘲笑或羞辱的风险 }\end{array}$ & \\
\hline $\begin{array}{l}\text { 能自然地告诉对方什么让 } \\
\text { 你获得性满足 }\end{array}$ & & 其他? & \\
\hline
\end{tabular}


时间：45分钟

材料: 黑板、粉笔

准备工作: 汶览步骤1所列出的行为, 以确保他们都适用于学生间的简短讨论。同时也确保你能够给 每个行为以简明的定义。有些行为可能是你的生活背景中极罕见的, 学生们可能从学习 中、电影中、网络上对其有些了解

概述 : 学生们思考与婚姻和家庭结构有关的习俗是否发生了变化以及如何变化的

目的: 帮助学生理清对长期关系的期望和价值观; 了解社会规范是会随着时间而改变的; 增强学 生的分析能力和写作技能

\section{步骤}

1. 将以下内容写在黑板上 :

- 童婚

- 同性关系或是同性婚姻

- 婚前性行为或婚前同居的

- 包办婚姻

- 丈夫欧打不顺从的妻子

- 婚姻咨询

2. 解释 :
- 家族共居

- 男性花费更多精力扮演父亲角色

- 一夫多妻

- 离婚

- 彩礼/家妆

\section{活动31}

在两性关系中我

们如何共处

- 这是一份各地婚恋风俗的清单。这其中的一些一一像离婚、同性婚姻以及结婚年龄下限一一是法 
律所规定的。另外对于一些行为一一如家族共居、婚前同居、父子模式一一通常没有正式的规定。你 可能赞同或反对其中一些习俗;

- 汶览列表, 然后选出一项你认为以后将会改变的习俗。你认为这个改变是好是坏?

3. 让学生们打开他们的笔记本。关于这些改变，让他们写下至少半页内容。提出以下问题来激发他们 的思考 :

- 你认为列表中的哪一个习俗会在下一代有所改变?

- 你认为这些改变会发生在我们的文化中, 还是其他地方?

- 你预期人们的态度会发生改变? 是关于这个习俗的普遍性方面的变化吗? 或者这个习俗方式会变 得不同? 还有其他方面吗?

- 你认为关于习俗的法律会改变吗?

- 讨论是否赞同你所预期的变化? 你的观点是基于生活经验还是见闻?

4. 过一遍整个列表。请写某个习俗的同学自愿分享他写的内容。然后让其他人进行简短评论，根据时 间的安排来决定是否关于每个习俗都找人回答

5. 留出最后5分钟讨论：

- 你认为是什么使得整个社区或甚至一些国家改变它们的婚恋观念?

- 人们从哪里获知新的观点, 这些观点是如何流行起来的? (探讨: 选择不同生活方式的人; 媒体; 文 化间的交流；经济情况变化影响家庭成员的居住地；政治运动）

- 想象你有个笔友居住在完全不同的文化环境中。你笔友居住的地方的习俗和我们这儿的十分不同。离 开教室时, 想一想你要怎么向你的笔友描述, 在我们这里, 人们是如何寻找长期伴侣的、居住在一起 要做什么安排、我们一般怎样开始或结束一段婚姻? 
时间：步骤1一-3：40分钟 步骤4：5分钟（至少在进行第5-6步的前一天进行）步骤5-6: 40 分钟

材料：黑板、粉笔

准备工作：这是一个分成两部分的活动（第1-3步和第4-6步）; 你可以开展其中一部分或全部。决 定好你会进行一部分还是全部, 如果你会进行地4-6步, 要确保在第4步要在第5-6步至少 前一天完成

概述: 学生列出他们希望在长期伴侣身上看到的特质, 并从性别角度分析自己的观点。学生们从 诗和歌曲中想象出的未来伴侣

目的: 让学生能够认清并思考长期伴侣身上他们最看重的是什么特征; 理清他们的价值观, 并增 强他们的抽象思维能力和写作能力

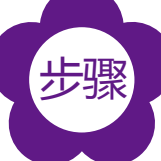

1. 解释 :

- 今天我们要讨论什么是长期伴侣身上所应具备的品质或特征;

- 拿出你的笔记本, 写下至少5条你认为在配偶或长期伴侣关系中重要的品质或特征。

- 将这些特征按重要程度排序, 第一个是最重要的。

2. 将学生按性别分为两组，(除非这个班上全是男孩或全是女孩)。如果你有很多学生，你可能要分 出两组女孩和两组男孩。解释 : 
- 将你列出的内容和组内其他成员的相比较。找出出现频率较高的品质, 用1、2、3来标注。(你会 发现不同的人会用不同的词来形容同一种品质, 例如, 慷慨/大方; 有趣/幽默; ）（教师在教室内 转一圈, 向学生提供帮助。)

- 在每组中选出一个人, 让他在黑板上写下所在小组认为重要的三四个特征。记下这些特征是由男 孩还是女孩写下的。

3. 汶览所写下的特征然后让大家就以下问题展开讨论：

- 你从男孩和女孩写下的内容中看出了什么?

- 它们在哪些方面相同?

- 它们在哪些地方不同?

- 你认为为什么会产生这些不同?

- 女孩们对于男孩所写下的内容有疑问吗?

- 男孩们呢?

- 你察觉所写的内容中有没有关于性别的刻板印象? 如果察觉到了, 这种刻板的观点是什么?

4. ( 这一步必须在第5-6步前至少一天进行，如果可能的话，将任务安排写在黑板上 ) 解释创造性写作 方面的任务，步骤这一任务会在课上进行还是被留为作业

- 想像你愿意与什么样的人共度余生, 或者希望选择什么样的人作为长期伴侣;

- 为那个想象中的人写一首诗, 或歌曲;

- 写下你希望从那个人身上, 或者从长期关系中得到什么? 包括你期望的生活、你愿意被怎样对待以 及什么是你不能忍受的; 
- 或者, 如果你不希望今生拥有长期关系, 你也可以写一首诗或歌曲来步骤;

- 明天 (或下次课)，你将有机会朗读或表演你所写的内容。

5. 确定一个表演/诵读日，让学生们分享他们写的信件、诗词和歌曲。如果你没有时间让每个学生都朗 读或表演，让同学自愿报名。你也可以让同学们将作品交给你阅读，并且不需要他们在作品上署名。

6. 留出10分钟，让同学们就以下问题进行讨论 :

- 你听到的哪一点最能打动你?

- 男孩和女孩希望在伴侣中获得的东西有所不同, 这些不同是如何影响他们与伴侣的关系的?

- 你是如何解释这些不同的? 也就是说, 人们对于各种特征的排序是基于什么考虑?

- 每个人都需要一段长期关系才能活得开心吗?

- 为什么你认为现在思考你想要 (或不想要) 什么样的关系非常重要? 


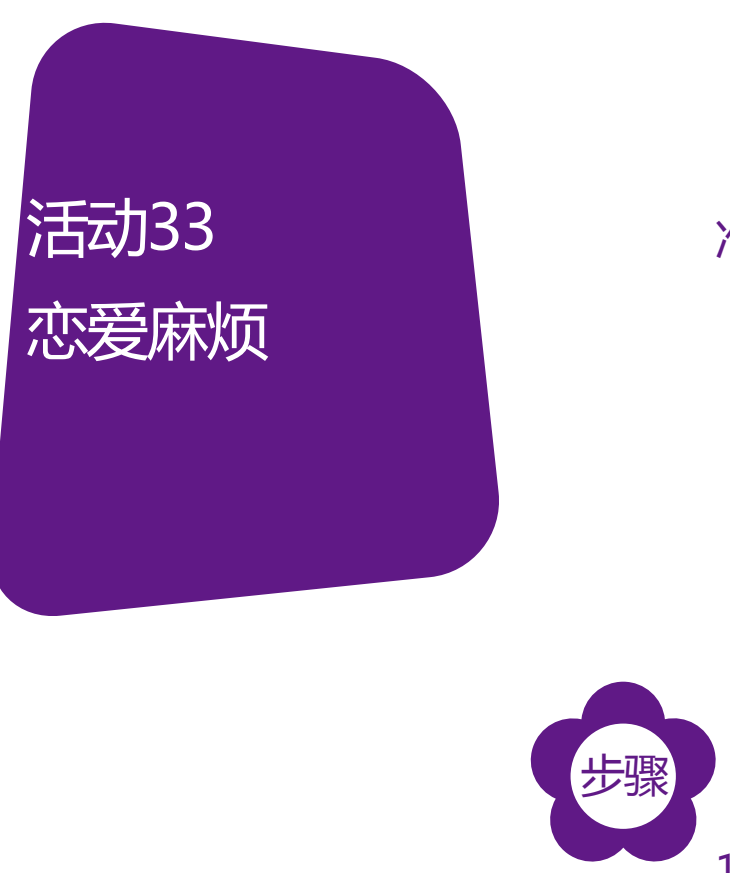

时间：步骤1-3: 30分钟 步骤4-6: 50分钟

材料 黑板、粉笔；四个关于恋爱关系的故事（来自 “麻烦的恋爱关系的案例” ），每个故事 写在一张单独的纸上

准备工作：从案例研究中选出 4 个对于你的学生来说最合适的。对它们做必要调整后将它们分别复 印。搞清楚如果一个人面对一段麻烦的关系或在一段关系中遭受暴力, 他们该向何处寻求 帮助。( 参见第6 步的最后一个问题 )

概述: 学生们阅读完这些关于处于困境关系的故事, 讨论他们决定结束一段关系的原因。（注 释: 在这次活动前, 学生应该已经学过, 人们有权利摆脱一段充斥暴力的亲密关系。1

目的：能够使学生找到处理关系中的麻烦以及结束一段关系的策略。弄清楚在一段关系中, 他们 不能容忍哪些行为; 澄清他们对于一段关系中个人尊严的价值观; 培养合作学习能力并使 他们逐渐适应当众讲话

1. 解释 :

- 今天我们将探索恋爱关系中的问题;

- 哪些是人们经常在浪漫关系中面临的问题? (将回答写在黑板上, 例子可包括: 家庭牵绊、交 流、不对等的期望和承诺、嫉妒、经济力量和决策权的冲突 )

2. 将学生分为四组。向他们解释，

- 我会给每组一个故事的开头, 这个故事是关于一段关系的。每一组将自行决定这个故事将如何发 展下去。决定这对伴侣在可能的情况下该怎么做;

- 准备好将你的故事结局以5分钟短剧的形式演出来。当你们准备的时候, 想象并演出这对伴侣 的对话; 
- 每组中的两个人将扮演这对伴侣, 其他人要贡献意见、扮演其他角色, 例如家庭成员。

3. 给每组一个关系的情景，并留出时间让他们准备

4. 让一组演出他们的故事。给学生几分钟时间来讨论

例如

- 这对伴侣间存在着怎样的问题和冲突;

- 他们是如何对付他们的问题的;

- 你认为这组同学是如何解决问题的? 这个结果现实吗;

- 有没有人提出什么其他的解决方法。

5. 以相同的方式进行其余几组。给每组留出10分钟 (表演和讨论)

6. 以针对以下问题的讨论来结束活动 :

- 有哪些迹象警示一段关系中出了问题;

- 什么情境可能使得一个人停留在一段麻烦的或者暴力的关系中（例如：子女、经济需求、无颜 离婚 ) ;

- 有时人们结束一段关系, 包括婚姻。人们对待离婚的人与对待已婚的人的方式相同吗? 如果有, 提 问: 为什么不同? 你如何看待这种不同;

- 什么情况或行为会使你结束一段关系;

- 在一段关系中遭受暴力的人可以向谁求助? 当一个人在一段关系中面临困境, 可以向谁寻求帮助或 向谁咨询。 


\section{案例研究材料 \\ 处于困境关系的案例研究}

安迪和百图高中时结识并开始交往，现在他们要去上不同的大学，他们在家乡过假期。昨晚他 们和朋友们一起出去时, 百图对安迪的一些评论伤害了她的感情。她看起来非常沮丧, 这时百图说 他刚才只是开玩笑的。过了一会儿, 安迪问到一个他总是在提的女人, 他承认他和那个女人发生了 性关系……

卡拉和达欧德结婚一年了。他们经常爆发激烈的争吵，但他们从没想过要分手，直到上个月。 他们因为卡拉的家庭大打了一架, 达欧德抽了她几巴掌。第二天他表现的十分歉疚, 还送了她件礼 物, 承诺这种情况再也不会发生。但是昨晚他食言了。今天早上当卡拉醒来时, 她的眼圈是乌青 的, 眼睛肿得睁不开……

依莱娜和弗里兹谈了几年恋爱了。他们曾经共度一段美好的时光一一他们非常开心, 无所不 谈, 常有肢体接触。现在他们似乎对对方厌倦了。他们 (在一起) 绝大多数时间是在看电视, 几乎 不说什么话, 甚至性生活也变得乏味而稀少。依莱娜纳闪，“我们的爱情怎么了? ”

克里特和亨利一年前开始约会。克里特十八岁, 刚刚开始她的大学生活, 亨利二十五岁, 正在 工作。克里特对于进大学很兴奋, 期望着结交新朋友, 努力学习, 享受校园生活, 感到十分兴奋。 亨利感到他准备好了稳定下来建立一个家庭。他想和克里特结婚。他最近提到了婚姻, 克里特的反 应让他震惊。她看着他, 那神情好像他疯了, 说道, “我刚刚开始我的学业, 我现在想享受生活、 努力进取, 而不是结婚!”

伊凡和何塞法成为伴侣一年多了。他们都有工作, 也都喜欢和朋友们一起出去。昨晚, 他们去 了一个俱乐部。何塞法和她的女朋友们说笑, 还一起跳舞。伊凡和很多人跳了舞, 并和一个刚认识 的女人聊得很投机。他认为自己和何塞法都玩得很尽兴。但是当他们回到家时, 何塞法显得很生 
气。她说, “我看到了你对那个女人很特别。别跟我说你对她不感兴趣! 还有, 你们亲热的跳舞! 就想让那儿的所有人都注意到你! ”

奇奇是大学生，她有一份兼职工作，但挣得不多。丽莎有一份技术性工作，报酬很不错，所以 她负担了她们的大部分花销。奇奇和丽莎达成协议, 可能的话奇奇会去买生活用品并负担一部分房 租, 但这种情况极少发生。奇奇给自己买衣服的时候很舍得花钱, 这困扰着丽莎, 因为她觉得这是 没必要的。几天前奇奇拿到了假期打工的薪水, 昨天她就把这些钱的大部分花去买了台电脑, 没跟 丽莎商量, 当她拿着电脑回家时, 丽莎非常生气。

米卡和诺布是夫妻并有三个孩子, 分别是 1 岁、3岁和6岁。当米卡月经迟了的时候她去看了医 生, 然后发现自己已经怀孕5周了。米卡听到这个消息感到很沮丧。她和诺布勉强过日子, 他们手 头并不宽裕。她还很生气。她怀疑自己是在诺布喝醉了回家, 没用安全套的那晚怀上的。她告诉诺 布她想做人工流产。诺布十分震惊。他说，“我知道这很难，但我不希望你那么做……” 


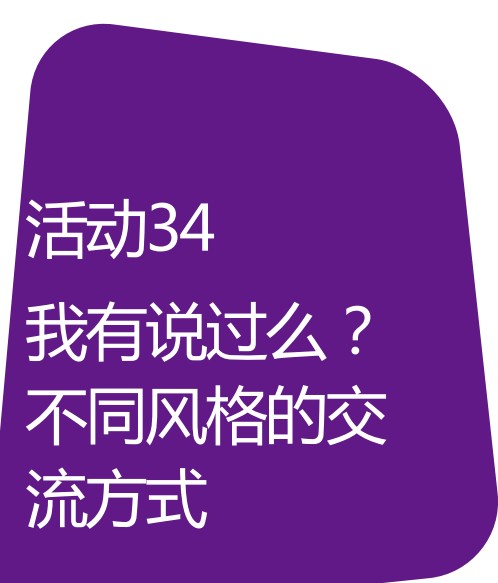

时间：45-60分钟

材料 准备给每位学生的两张工作表（“自我评估”和“分析回应”）的复印件 准备工作：准备材料的复印件或者在活动时读出这些内容

概述：学生分析让人不舒服的口头回应, 并且分辨出哪种是攻击性的, 哪种是积极的, 哪种是被 动的回应方式。他们填写一个关于自己在让人感觉不舒服的情况下如何回应的调查问卷

目的：提高学生对于不同风格交流方式的理解和做出积极回应的能力; 提高他们的分析和对 话技巧

1. 介绍活动 :

- 今天我们会学习到不同风格的交流方式;

- 特别是当我们处于一种让自己感觉特不舒服的情况下时, 我们可能无法清楚地、以我们想要的方 式去交流；

- 哪些因素会影响我们用最好的方式表达自己的愿望?

探讨以下原因并写在黑板上:

我不知道如何清楚地表达自己的想法;

我怕如果我不同意的话，其他人会对我看法不太好;

我怕我说的话无人听, 我说了也没用;

我怕我会发脾气; 
我发现靠吓唬人较容易。

- 首先, 我们要找出攻击性的、被动型的以及积极型的交流方式的不同之处。接下来你需要填一份调 查问卷, 这份问卷是关于在让人不悦的交流情形中，你自己的回应方式的;

- 你觉得被动的与咄咄逼人的交流方式有何不同? 我们所说的 “积极” 的交流方式是指什么? (探讨 几种交流方式: 诚实、直接; 说出你的感受和需求; 使用积极的肢体语言; 为自己说话; 清楚但不 带有咄咄逼人意味的交流。）间接的交流方式是指什么;（探讨：不完全被动但并不直接）

2. 让学生结成对并分发 (或者大声读出) “分析回应” 表格

解释 :

和你的同伴一起阅读 “分析回应” 表格中左栏的关于冲突情况的描述。接下来阅读右栏不同的回应 方式。检查每一种回应方式是咄咄逼人的、被动的或者积极的。对于冲突5, 写下一种冲突情形和 对于这种情形的不同回应方式。

3. 回顾每一种冲突，要求学生提供正确的答案并让他们评论当听到进攻性回应与积极回应时的感受 ( 答案 : 回应a,f,i和 $k$ 是咄咄逼人的。回应 $c, d$ 和h 是被动的。回应 $b, e, g$ 和j是积极的。)

4. 分发 “自我评估” 表并让学生自己独立填写。（或者在黑板上写下这些表述和可能的回应，大声地 读出来，让学生写下他们自己的回应。）

5. 通过以下问题结束讨论 :

- 对于你自己的交流风格, 你了解到了什么;

- 学习如何积极地交流有用么。

学生工作表

自我评估 : 我在感觉不悦的情况如何交流? 
当我与一个男孩处在令人不悦的情况下时, 我会表现得:
A 被动
C 积极, 充分尊重
B 咄咄逼人
D 间接或巧言应对

当我与一个女孩处于不悦的情况下时, 我会表现得:
A 被动
C 积极, 充分尊重
B 咄咄逼人
D 间接或巧言应对

当我与一个成年男子处于不悦的情况下时, 我会表现得:
A 被动
C 积极, 充分尊重
B 咄咄逼人
D 间接或巧言应对

当我与一个成年女子处于不悦的情况下时, 我会表现得:
A 被动
C 积极, 充分尊重
B 咄咄逼人
D 间接或巧言应对

当我与一个我认为和我拥有不平等地位的人处于不悦的情况下时, 我会表现得:
A 被动
C 积极, 充分尊重
B 咄咄逼人
D 间接或巧言应对

对我来说，我会这样表现我的愤怒：

A 较为随和 (但我有时会有攻击性的回应)

B 较为随和 (而且我从不咄咄逼人或者凶猛地回应) 

C 不随和也不执拗
D 有些或者非常执拗

对我来说, 当我感觉容易受到攻击或者处于弱势时, 我会这样表现:
A 较为随和 (但我有时会有攻击性的回应)
B 较为随和 (而且我从不咄咄逼人或者凶猛地回应)
C 不随和也不执拗
D 有些或者非常执拗

分析回应

\begin{tabular}{|l|l|l|l|l|}
\hline 冲突 & 回应 & 咄咄逼人的 & 被动的 & 积极的 \\
\hline $\begin{array}{l}\text { 1. 一个男孩和一个 } \\
\text { 女孩打算发生他们 } \\
\text { 的第一次性行为, } \\
\text { 但是他们没有安全 } \\
\text { 套。其中一个说: }\end{array}$ & $\begin{array}{l}\text { A.据我所知, 你感染了艾滋病病毒! } \\
\text { 笨蛋! }\end{array}$ & & & \\
\cline { 2 - 5 } & $\begin{array}{l}\text { B.我也想, 但是除非使用安全套。我 } \\
\text { 知道哪里可以获得它们。 }\end{array}$ & & & \\
& C.好吧, 应该没问题。 & & \\
& & & & \\
\cline { 2 - 5 } & & & & \\
\hline
\end{tabular}




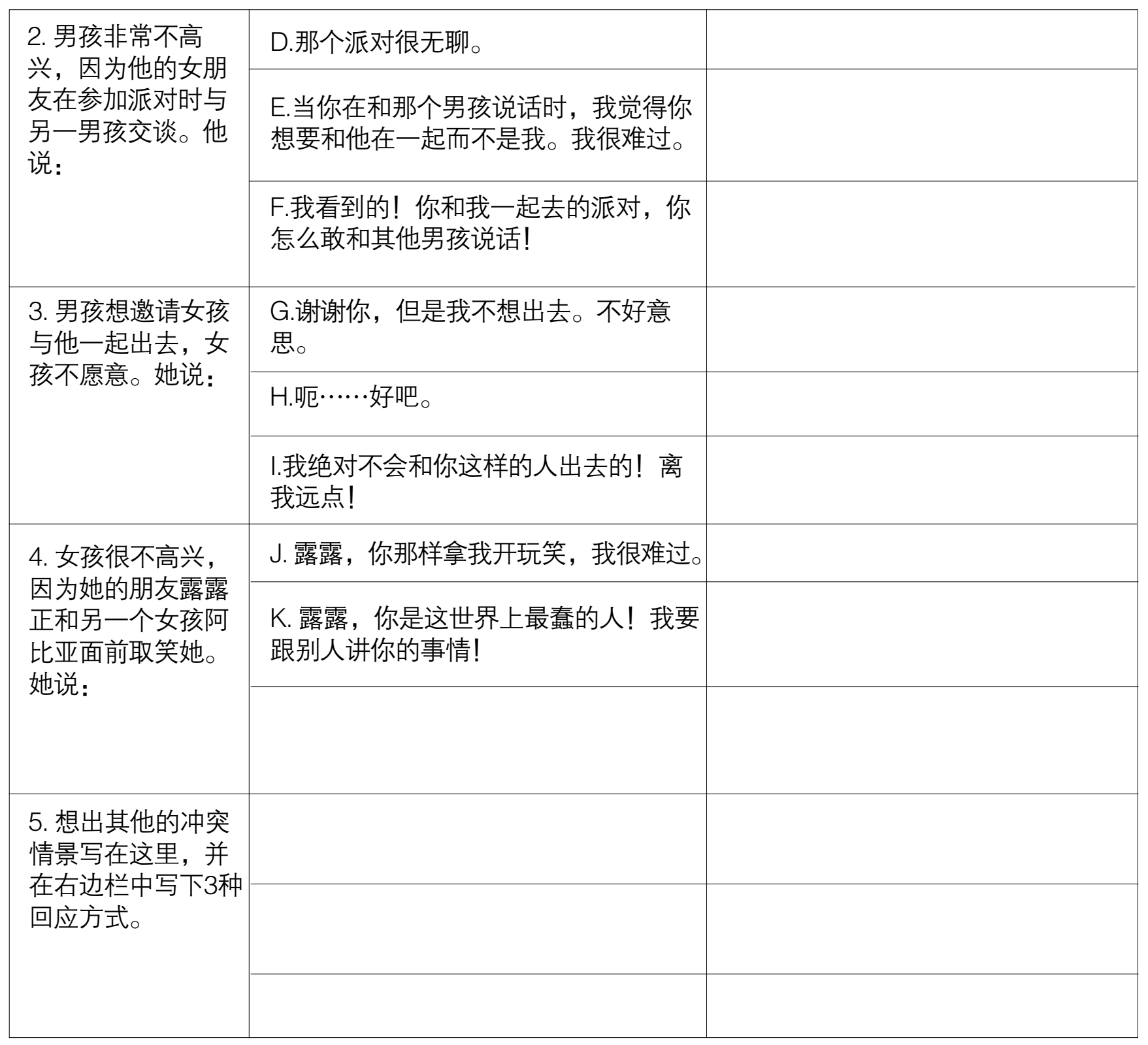


时间：50-60分钟

材料：黑板、粉笔; 提示卡和课堂活动材料

准备工作 : 复习《指导手册》上第五单元里 (或是你自己的教材上) 关于有效沟通的信息。复习步骤 6 中特定行为的清单, 并且如果人们可接受的行为在你们的文化中有着不同, 你可以进行 修改（例如，眼神交流在某些文化中被认为是不礼貌的; 摇头也可能有着不同的含义）。 找出一两对比较擅长角色扮演的志愿者并为他们准备提示卡 (可查看提示卡的样本)。如 果能让志愿者提前一天准备并彩排会效果更为理想。最后, 注意你自己的倾听技巧一一特 别是在这个课程中

概述：学生练习促进（或者阻碍）交流的行为

目的：让学生学会促进交流的行为

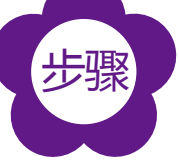

1. 在每一个小短剧里，给每一个扮演角色的志愿者一张提示卡。如果有必要的话，在你进行步骤2时， 给他们几分钟的时间准备或排练

\section{2. 通过解释向其他人介绍主题}

- 我们通常说交流是一个双向的过程;

当一个人在说的时候, 另一个在做什么? (听) 所以, 倾听也是交流的一个部分? (是的) 今天我 们将要探索, 当我们在倾听时应做何种交流。我们将会学习到 “积极倾听” 在人际交流中起到的重 大作用。 
- 我们将从一个演示开始。拿出你的笔记本, 观察听者并记录下你观察到的每一个你认为会阻碍有 效交流的行为。

3. 让志愿者表演 “消极” 交流的小短剧 ( 短剧\#1 )

4. 通过提问思考这个短剧 :

- 你会怎样评价这个谈话? 它是否有效并让人满意;

- 这个 “听者” 有哪些语言的和非语言的行为; (在黑板上, 写下学生提到的每一个消极的行为, 并探讨以下：）

非言语的行为 :

○ 皱眉头;

○ 不屑一顾的样子, 望着天转动眼珠;

○ 摇头表示你似乎想说 “不” ;

○ 看远处或者移动到远处, 表现得不感兴趣或觉得无聊, 打呵欠。 言语的行为 :

○打断说话的人, 表现出不耐烦, 表现出嫌说话的人说太久了;

○ 对说话者进行消极的评论，例如 “你错了” 或者 “真是个愚蚌的主意” ;

○询问 “说者” 当不被他人倾听时感觉如何。

5. 让志愿者表演 “积极” 交流的小短剧 (短剧\#2)

6. 通过提问思考这个小短剧：

- 你怎样评价这个谈话? 它是不是更令人满意; 
- 有哪些言语的和非言语的行为让这次谈话比之前那次更成功?

(在黑板上写下学生提到的积极的行为并探讨以下：)

非语言的行为 :

○ 进行眼神交流;

○点头 (表示你似乎在说 “对” ) ;

○ 朝说话的人稍微倾斜一点以显示你在听;

○展示微笑或者轻拍对方的背。

言语的行为 :

○ 要求对方解释, 确保你很好地理解了说话者;

○ 表现出想听到更多的兴趣（例如, “能不能跟我讲讲那个……” );

○认同说话者的感受 (例如, “我能理解你的感受” );

○认同说话者的想法 (例如, “好主意” );

○ 避免作任何让说话者感觉自己被他人否定的陈述;

○ 感谢说话者对你的信任并分享他或她的想法。

7. 让学生三人一组练习积极倾听

解释 :

- 在每一组, 每人要说 2 分钟。当轮到你说的时候, 你说一些你比较关注或者沮丧的事情。为了达到 练习的目的, 选择一个不是那么私密的、你可以很自在地与同学谈论的话题。你可能会感觉到充满 
希望, 生气, 忧虑, 受伤, 或者焦虑。如果你不知道想要说什么, 练习说服别人投票选举你为青 年理事会的领导;

- 第二个人会练习积极倾听, 第三个人会观察并给予反馈。通常情况下你会给听者反馈, 但你也可 以给说话者反馈。2分钟过后, 轮换你们的角色。然后是第三轮。通过这种方式，你们每个人都可 以扮演其中的三个角色。

8. 开始第一轮

- 说话者告诉听者你生活中的一些担忧的事情

- (2分钟过后：) 停下来。观察者进行60秒钟的反馈。

9.1分钟后: 开始第二轮

- 说话者, 你变成了听者。听者, 你扮演观察者的角色。观察者, 你变成了说话者;

- (2分钟过后：) 停下来。观察者进行1分钟的反馈。

10.1分钟后: 开始第三轮。当学生在完成这一轮时，你不妨在黑板上写下如下句子去鼓励他们思考， 这句话是内科医师作家卡尔.曼宁格 (Karl Menninger) 说的：“倾听是一件有吸引力而且新奇的事 情，是一种有创造性的力量。倾听我们的朋友，我们会离他们更近。当我们被倾听，它创造我们，让 我们放开、舒展自我。

- 再一次轮换角色重新开始;

- (2分钟过后：) 停下来, 观察者给出反馈。

11. 预留10分钟作为讨论时间，从以下问题中选择：

- 当你是说话者时, 听者如果没有很好地听你说话, 你会感觉如何;

- 如果两个人真的发生了冲突, 而且都很心烦意乱, 那会怎样? 这会让交流更困难还是更容易; 
- 一些研究者发现男性和女性的交流风格是不同的。当女孩和和女人们感到沮丧时, 她们被教育要怎 样去表达自己;

- 当男人和和男孩们感到沮丧时, 他们被教育要怎样去表达自己? 人们能学会用不激烈或者非进攻性 的方式去交流么;

- 你认为说话咄咄逼人或用词激烈如何影响恋爱中青年男女的交流;

- 人们应该怎样去改进男性与女性之间的交流, 特别是当他们有冲突的时候;

- 思考你希望在你的交流风格中改进的一个方面, 在今晚和这个星期与其他人多加练习该方面的行 为。同样地, 在你尝试着表达自己想法时, 请注意人们是如何倾听的。

\section{课堂活动材料}

为 “差劲的聆听者” 短剧准备的提示卡 (\#1):

你们之中的一人会扮演 “说话者” ，一人会扮演 “听者”。

说话者: 想象一个情境并写一个剧本。选择以下的一个话题:

（1）（假装你）遇到了一些让你觉得很不公平的事情;

（2）假装你为自己的一个疯狂的计划或者想法感到兴奋不已。

这个短剧中, 你想要特别清楚表达自己的经历、想法或者感受。你将会花几分钟的时间尝试与你的同 伴谈论这些，并试着能够被倾听。预计说2至3分钟。

根据同伴听你的方式进行回应。如果你觉得他或她没有在听你说, 你表现出的失望, 提高你的声调, 或者是以任何在这种情况下你觉得自然的方式进行回应。如果对方认真仔细并且充分尊重地倾听, 用 
相应的自然的方式表现。

聆听者: 你是个很差劲的倾听者。当说话者尝试着与你交谈时, 你展示出差劲的倾听技巧。你的任务 是尽你可能多地使用下面列出的消极的语言和非语言的行为:

非言语行为：

- 皱眉头;

- 不屑一顾的样子, 望着天转动眼珠;

- 摇头表示你似乎想说 “不” ;

- 看远处或者移动到远处，表现得不耐烦或显得无聊，打呵欠。

言语的行为 :

- 打断说话的人, 开始说关于你的事, 或者转换话题;

- 对说话者进行消极的评论（例如“错! ”或者 “真是个愚蚌的主意。”）。

为 “好的倾听者” 短剧准备的提示卡 (\#2)：

你们之中的一人会扮演 “说话者”，一人会扮演 “听者”。

说话者 (步骤与短剧\#1一样) : 基于一个想象的情景写一个剧本。选择以下一个话题:

（1）（假装你）遇到了一些让你觉得很不公平的事情;

（2）假装你为自己的一个疯狂的计划或者想法感到兴奋不已。

这个短剧中, 你想要特别清楚表达自己的经历, 想法或者感受。你将会花几分钟的时间尝试与你的同 
伴谈论这些，希望能够被倾听。预计说2至3分钟。

根据同伴听你的方式进行回应。如果你觉得他或她没有在听你说, 表现出你的失望, 提高你的声调, 或者是以任何在这种情况下你觉得自然的方式进行回应。如果对方认真仔细并且充分尊重地倾听, 用 相应的自然的方式表现。

聆听者: 你是一个积极的倾听者。当说话者在尝试着与你交谈时, 你展示出积极的倾听技巧。你的任 务是尽可能多地使用下面列出的积极的语言和非语言的行为:

非语言的行为 :

- 进行眼神交流;

- 点头 (表示你似乎在说 “对” ) ;

- 朝说话的人稍微倾斜一点以显示你在听;

- 展示微笑或者轻拍对方的背。

\section{言语的行为 :}

- 要求对方解释, 确保你很好地理解了说话者;

- 表现出想听到更多的兴趣（例如，“能不能跟我讲讲那个……”);

- 认同说话者的感受 (例如, “我能理解你的感受。” );

- 认同说话者的想法（例如，“好主意”）;

- 避免作任何的让说话者感觉自己被他人认定为陈述不充分;

- 感谢说话者对你的信任和分享他或她的想法。 


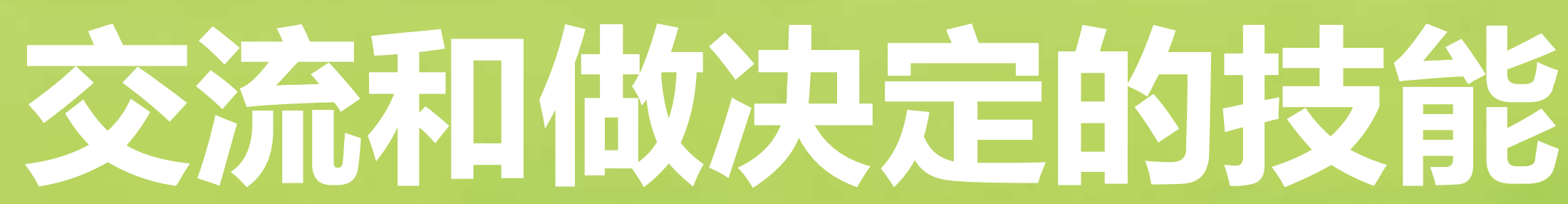




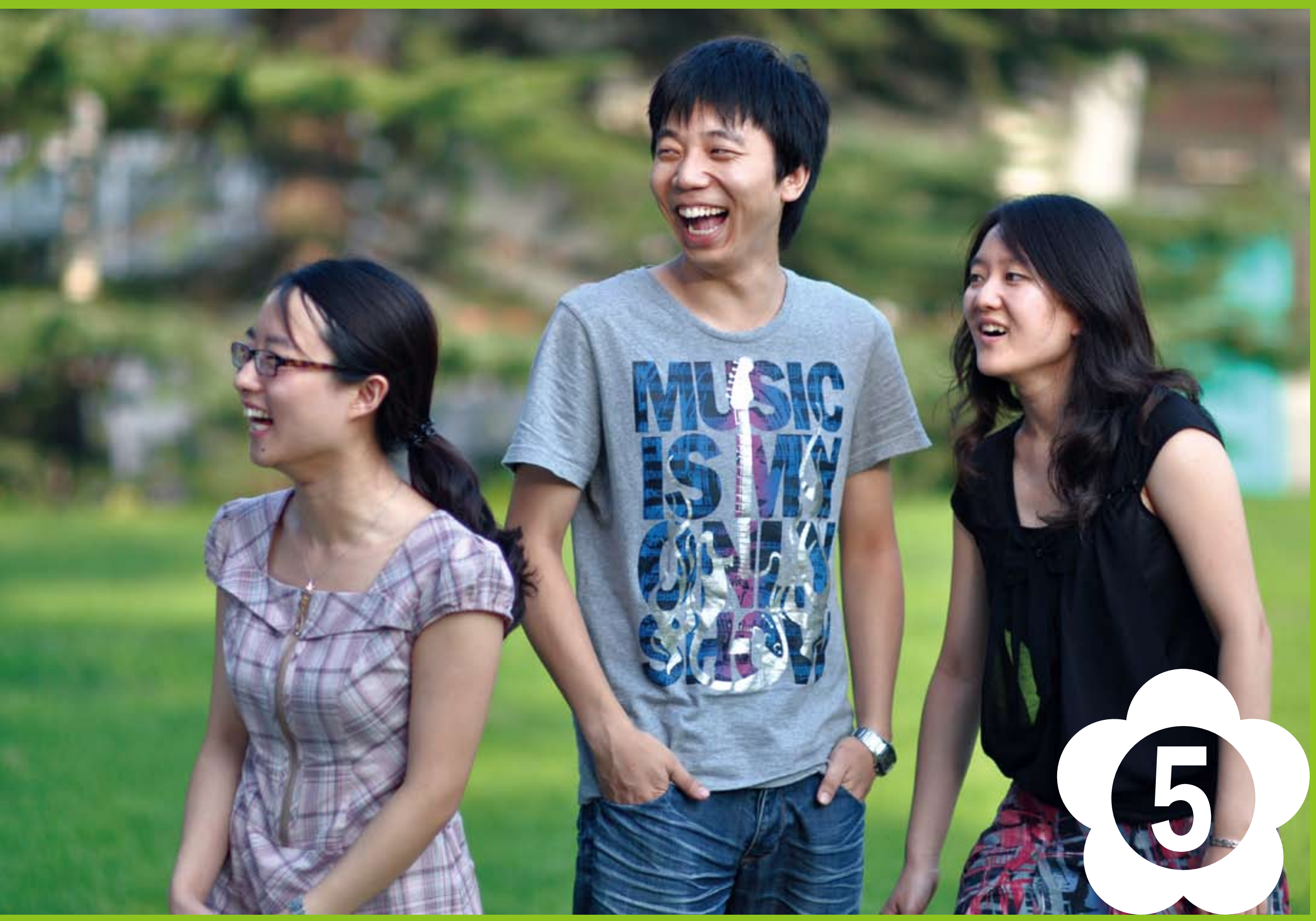




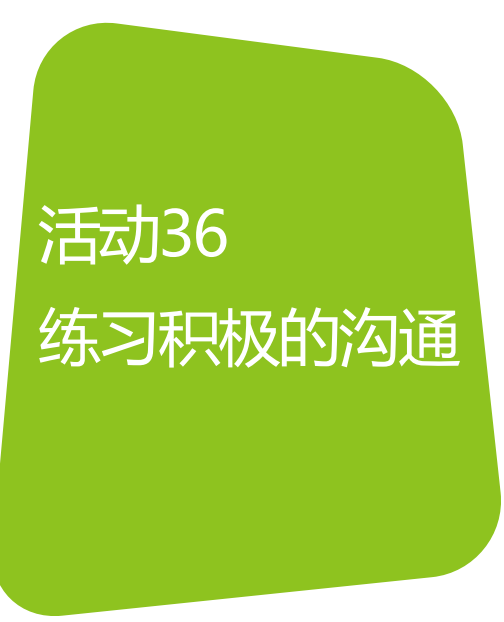

时间：30-40分钟

材料：提示单和回应范例的复印件

准备工作：阅读提示和回应的范例；你不妨修改其中的一些使他们更加符合文化背景

概述：学生阅读积极的行为表现, 讨论文化影响, 并且练习在权利被滥用的情况下主动的沟通

目的：让学生能够在他们有需要时更加积极地交流; 加强他们的对话技巧

\section{1. 用以下引导性的提问开始：}

- 今天我们将会学到更多的关于积极交谈的知识。有时候你很难清楚地说出自己想要的 (比如说在学 校里想要被他人考虑担任领导职务), 要说出你不想要的也很难 ( 例如, 你不想吃他人为你准备的 食物）;

- 谁能记起你不确定该怎样清楚地表达自己的渴望或愿望的例子; ( 注意是女孩还是男孩更加可能去 回答这个问题 )

- 说出来有时会很困难, 甚至是当情况对于我们来说有重大意义的时候, 或者是当物质和情感上的后 果可能很重要的时候。练习充分尊重他人，积极的交流方式是有很大帮助的;

- 拿出你的笔记本, 我会读出一系列 “提示性” 的问题或者描述。对于每一个问题或者描述, 写下一 种积极但不咄咄逼人的回应方式，使用完整的语句;

- 第一个提示是：“告诉我为什么我要投你的票选你为班长” ; 
- 谁能够分享一个积极的回应方式? (帮助学生找到比较合适的回应, 例如: “我相信我是最具资 格的班长候选人。我希望我能指望你的支持。” 请注意, 回应的句子要完整。)

2. 对于每一个提示, 重复这个过程 ( 详情见清单)

\section{3. 通过讨论如下问题进行总结}

- 有的人可能认为积极主动与他们的文化不相符, 或者是很无礼。在什么情况下一个人应该主动积 极地回应, 即使如此回应会被看成无礼, ( 探讨: 什么时候你会觉得自己的权利被侵犯了）

- 交谈积极主动的女孩与交谈积极主动的男孩相比, 她的待遇与男孩的待遇一样吗? 一个说话积极 的人, 如果此人是来自少数民族或少数种族的, 人们对此人的这样的看法会不同吗? 这样的偏见 是如何限制了人们维护自己的能力?

\section{( 教师参考资料 提示清单和积极主动回应的案例 )}

用下列描述来提示积极主动的回应。下面也包含了回应的案例, 万一学生对于做出合适回应感到困 难, 可以参阅。

1. 探讨: “你会让我抄你的卷子么? 老师不会知道的。”

回应范例: “对不起。我认为不该分享考试的答案。”

2. 探讨: 你去见你的上司并要求加薪。

回应范例: “老板, 我想与你谈谈关于我加薪的问题。”

3. 探讨: 告诉你的爸爸你想明年继续上学, 虽然他不愿意。 
回应范例: “爸爸, 我尊重你的想法, 但是我觉得继续我的学业对我来说是极其重要的。我想跟你谈 谈我的理由。”

4. 探讨: “你为什么不像其他人那样都喜欢运动呢? 你怎么啦? ”

回应范例: “每个人都不一样, 我喜欢阅读和音乐。”

5. 探讨: “跟我们一起去旧工厂后面呆一会吧; 警察不会去那儿的。”

回应范例: “不用了, 谢谢, 我不是很感兴趣。”

6. 探讨: “我是市长。我听说你要求使用本市的一座建筑作为你们的校外俱乐部? ”

回应范例: “是的, 市长先生。我们会很负责并很好地利用空间的。你想知道更多关于我们计划的信 息么?”

7. 探讨: “如果你可以说服你身边的两名同学与你一起制作壁画, 我们会让你们画这面墙。” (对班 上同学回应 )

回应范例: “如果再有两位同学加入我们, 我们就能去制作壁画。你们愿意加入吗? ”

8. 探讨: “我们准备实地考察, 你觉得我们去哪儿好?”

回应的范例: “我想去 而且我觉得其他人也应该认为那里很有趣。”

9. 探讨: “那个外班的新生朝这边走过来了, 我们绊倒他吧。”

回应范例: “算了吧, 他没对我们做过什么。”

10. 探讨: 现在考虑一下这个活动开始时提到的情况。你被问到，什么时候你想表达自己的希望时本该 更直接些。写下你在那种情况下有可能会说的。你不会被要求和大家分享这个回应。 
时间：45-60分钟, 取决于学生对这个概念是否感兴趣

材料 黑板、粉笔

准备工作：熟悉解决冲突的五个步骤, 并思考在生活中你会怎样运用它们。你可以改变一些冲突的例 子, 使它们更加适合你的目标人群。如果改变了, 确保先选出一个简单的例子, 并思考怎 样通过这个例子来展示五个步骤。学生在最开始使用这些步骤的时候会感到塩尤, 但使用 一些简单例子并且不断练习会使情况好一些 ( 即使年纪小一些的孩子也可以引导他们完成 这五个步骤, 但如果你觉得这个活动过于宽泛, 调整活动, 只集中在步骤2--4. )

概述: 学生学习并练习解决人际冲突的五个沟通步骤, 在这一过程中要做到清楚和充分尊重 (注意：这个活动的目的是培养进一步更高水平的交流技巧。）

目的：帮助学生提高在亲密的人际关系中解决冲突的沟通能力; 加强他们的分析和对话技能

\section{活动37}

解决冲突的五

个沟通步骤

\section{步骤}

1. 解释

- 今天我们将学习和练习解决冲突的沟通技巧, 我们会一起分析各种情形, 然后分组练习;

- 这是一个想象出来的供我们一起练习的情形: 荣觉得奇瑞在朋友面前取笑他, 荣非常难过, 他想 去找奇瑞谈谈。

2. 在黑板上写下第一个提示语 ( “当你___。”) 并讨论 :

- 想象什么具体的行为使荣难过。避免用 “你有一个很差劲的朋友......” 这种笼统的指控, 说说在这 个例子中使荣难过的具体行为; ( 探讨: “当你取笑我的时候......” 或是 “当我听到别人说你取笑 我…… 。在黑板上把具体的行为填入提示语的后半部分, 可使用组员提供的语句 ) 
- 为什么与他人沟通非常重要, 尤其是在有某些恼人的行为发生的时候;

- 尤其要注意当你对某些行为的看法不太确定的情况。例如, 你可以说: “或许我弄错了, 但是我听 说在别人面前取笑我。”为什么说改变自己对发生的事情的理解可能很重要?

3. 写下第二个提示语：“我想的是—“并讨论：

- 很多人会跳过这个步骤, 但这是一个极其重要的步骤, 它要求我们思考我们自己的回答和反应;

- 荣有可能想象或开始觉得的是什么? “当你在他人面前取笑我的时候, 我想（开始觉得）......” 你并不在意我的感受;

你有可能告诉了他人我的过去;

你对自己的感觉并不良好;

每个人都在嘲笑我。其他的回答?

( 要接受不同的回答, 这些答案可能都是合适的。然后写下学生举的某个例子来完成第二个提示 语。)

- 说 “我想（或我担心）你并不在意我的感受” 与说 “你是不是不在乎我的感受? ” 有什么不同?

4. 让一个学生读出前两个提示语，包括后面填进去的句子。然后写下第三个提示语：“而且那让我感 觉

按如下的话题讨论:

- 注意荣有可能经历的情绪。这不是他所想的, 而是他所感受到的;

- 如果你觉得你的朋友和其他人取笑你, 你会有什么感受? ( 探讨: 受伤, 被他人背叛, 生气, 尴 价, 孤独, 屈辱)

( 将提示的两三个描述感受的词语写在黑板上, 完成第三个提示语 )

5. 学生上来读出黑板上所有的句子。写下第四个提示语：“这让我想 
荣会想做什么。（提示可能的回应, 例如, “这让我想：跟其他人讲你坏话; 大叫；跟你绝交; 呆 在家不想再见任何人。”)

( 用提供的一、二个回应完成第四个提示语。提醒学生, 我们在某个特定的时刻可能做出的事情通常 与我们计划要做的是不一样的。)

\section{6. 请一名学生读出黑板上所有的句子, 然后写下最后一个提示语：}

“但是我仍然 。”根据下面的话题讨论：

- 在某些情况下, 一个人会感觉到被侵犯了以至于他或她不想再跟对方沟通了。但是通常来说, 说话 者还是想继续维持与对方的关系。“听者” 听到他或她的话会有什么感受;

- 荣怎样才能有一个简单且直截了当的关于他自己感受的表达（提示类似的回应：“我仍然很在意 你” ; 或 “我还是想和你做朋友”。)

( 在黑板上写下一个合适的回应完成最后一个提示语。)

7. 读出所有的对话。让大家来作出一些评论

8. 让学生结成对, 然后解释:

- 现在你们自己练习这些步骤了。想出一个你曾经处理得并不好的冲突;

( 如果没有人可以想出合适的情景, 你可以从下面建议一个情况 )

$\sqrt{ }$ 你的爸爸病得很重, 但你最好的朋友并没有表示特别的关心;

$\sqrt{ }$ 你的表弟告诉你的朋友你爸爸进监狱了;

$\sqrt{ }$ 你的朋友不愿意与你一起分享教科书, 虽然你丢了教科书而且没办法再买;

$\sqrt{ }$ 当你想和朋友一起做些事情的时候, 他或她总是迟到; 
$\sqrt{ }$ 在一个派对上你的女朋友大部分时间都在和另一个男孩交谈;

$\sqrt{ }$ 你的男朋友说，如果你真的爱他，你必须和他做爱;

$\sqrt{ }$ 你的朋友在给你压力让你 “失身” ;

$\sqrt{ }$ 当你想使用安全套的时候, 你的女朋友生气了。

- 首先练习的那一个人需要告诉他或她的同伴要处理哪个冲突;

- 使用这五个提示语去处理冲突。用五分钟左右的时间回顾下所有的步骤, 一步一步来, 不 要担心开始的时候会觉得尴伧。看看你最后会有什么感受;

- 当你是听者时, 帮助你的同伴记忆对每一个步骤我们讨论了些什么。

9. 再次回顾五个步骤, 确保学生们能轻松自如地运用它们:

- 当你—( 特定的行为);

- 我想的是 (或我开始觉得)

- 而且那让我感觉 （描述情感的词语）：

- 这让我想

- 但我仍然 你。

10. 注意时间，保证每个人都有 5 分钟的时间练习

11. 鼓励讨论：

- 与你第一次面对这样的冲突相比, 这次你能够更好表达自己感受了吗;

- 这些语句有助于你表达你的真实感受么;

- 当你确认并说出自己的感受时, 你觉得如何;

- 记住你有被尊重对待的权利。当你觉得自己没有受到尊重时，你应当维护自己权利。如果你都不争 取, 谁会替你争取? 
时间：步骤1-4：40分钟 步骤5-6：30分钟

材料 黑板、粉笔; 透明胶带和马克笔; 海报板或者大白纸; 短剧和海报任务

准备工作：阅读短剧和海报的任务书, 按照需要进行修改使其更加适合目标人群。如果你打算将活动 分成两个部分, 决定好在哪个地方进行划分。将每一个短剧和海报的任务书写在单独的纸 上, 在活动时分发给不同的组。思考怎样提高短剧的戏剧性, 例如通过击掌并说 “再试一 次!"

概述：通过讨论和角色扮演, 学生练习语言的和非语言的技巧. 这些技巧对于交流性行为、做与 性有关的决定，以及健康问题，很有必要

目的：加强学生交流与性有关的决定和健康问题语言上的和非语言上的技巧; 增强分析和对 话技能

\section{步骤}

1. 通过提问来介绍本活动

- 青年人会与自己的朋友谈论性话题, 媒体也提及性话题, 大多数青年人与自己的男朋友或女朋友 谈论这个话题是容易还是困难;

回应一个正在进行性暗示的人是容易还是困难;

-为什么学会与有性行为和性健康问题的人交流很重要; ( 探讨: 表明你是否想有性接触; 哪种接 触让你觉得自在, 对于其他人来说呢; 预防性传播疾病（包括艾滋病病毒）; 弄清楚与怀孕有关 的打算; 防止意外怀孕。） 
- 结成对子并分成小组。8个学生（四对）表演1分钟的短剧, 练习如何使用积极的交流方式去处理 这些不同类型的情况。其余的学生分成4个小组, 制作并展示海报; 每张海报要展示3种开启一个 困难话题的方式。

2. 结对、分组，并分派短剧和海报的任务

3. 让每一对同学表演他们的1分钟短剧。对于每一对，只要其中的甲先 “放弃”，马上说 “停! 再试一 次! ”这个提示就是让表演者再次表演，用更为积极的方式沟通

4. 4 个短剧结束后, 鼓励讨论

询问学生:

- 你们觉得哪个版本（消极被动的或主动的）更常见;

- 你看到的东西中你觉得什么比较有帮助;

- 对于处理这个谈话, 你们还有其他的建议吗?

- 我们希望这个谈话将朝怎样的方向引导?

5. 让每一个制作海报的小组展示他们的海报。让小组中的一个人来展示海报, 另一个人回答问题, 并 征集评论和建议 ( 你不妨让海报在墙上多贴几天)

\section{6. 通过下面的讨论问题结束 :}

- 我们已经看见了在一个与性有关的情境中, 如果对方的地位或者力量比你强大, 维护自己会变得 更加困难。记住在涉及性行为情况下，你始终是有权利去同意或者拒绝的

- 我们怎样准备开始或排练一个与性有关的较为困难的对话? (探讨：在镜子前预演; 先写下你想 要说的 ) 
第一对 (男女搭配)：

展示一个关于防止性传播感染的1分钟对话。甲提出这个问题, 乙比甲年纪稍长, 表现得不合作。学生 可以自行决定哪个角色是男性哪个是女性。这个场景将表演两次。第一次, 展示甲让步了, 同意不使 用安全套, 当老师打断后, 停止表演, 开始第二次表演, 展示甲怎样能坚持这个话题不让步的。

第二对 (男女搭配)：

甲提出关于预防怀孕的话题。乙想要有个孩子, 所以拒绝甲希望避孕的想法。学生可以自行决定哪个 角色是男性哪个是女性。这个场景将表演两次。第一次, 展示甲让步了同意不进行避孕。当老师打断 后, 停止表演, 开始第二次表演, 展示甲是怎样能坚持这个话题不让步的。

\section{第三对 ( 男女搭配 )}

甲和乙是同班同学; 你们可以自行决定哪个角色是男性哪个是女性。当他们一起往学校走时, 甲总是 将手臂搂着乙身体。但是乙并不喜欢这样。这个场景将表演两次。第一次, 乙不高兴但并未有回应。 当老师打断后, 重复这个场景, 展示乙是怎样果断地回应的。

\section{第四对 ( 男女搭配 )}

当一个女孩（乙）正往社区中心走时，甲（社区中心的一个成年男性领导）将手臂搂着她。这个场景 将表演两次。第一次, 乙不高兴但是并未有回应。当老师打断后, 重复这个场景, 展示乙是如何果断 回应的。 


\section{海报任务}

\section{海报1:}

讨论如何告诉你的亲密伴侣, 你可能有性传播感染的疾病。制作一张海报展示开启这个话题的3种方 法。海报里可以有绘画。

海报2:

讨论如何告诉你的男朋友或者女朋友你想做爱, 并且想知道对方是否也有同样的感受。制作一张海报 展示开启这个话题的3种方法。海报里可以有展示两个人谈论这个话题的插图。

海报3:

讨论一个人怎样才能与父母或信任的成年人开始一段关于报告遭受性侵犯的对话。制作一张海报展示 开启这个话题的3种方法。海报里可以有展示两人讨论这个话题的插图。

海报4:

讨论一个有过性行为的人应该如何告诉自己的性伴侣, 他想继续维持和对方的关系, 但是不想再有性 行为。制作一张海报展示开始这个话题的3种方法。海报里可以有展示两人讨论这个话题的插图。 
时间：步骤1-5: 40 分钟 步骤6: 80分钟

材料 黑板、粉笔、纸张和现成的绘画材料 (彩笔、马克笔、钢笔)

准备工作：阅读《指导手册》第五单元关于实施决定的章节, 或你的教材中相关章节。通读 “困难决 定示例” 的学生阅读材料, 并挑选出你想用的范例, 或自己编写合适的范例。可能的话, 将实施困难决定的 8 个步骤和困难决定案例给每个学生复印、分发一份 (也可将两份材料 订在一起 )

概述: 学生学习执行决定的基本步骤, 创作一幅漫画描绘他人执行困难决定时的情况

目的：提高学生轻松并深思熟虑地执行困难决定的能力; 增强分析和对话的技巧

1. 通过提问介绍主题

- 想一想, 有一次你做了决定, 但是要执行它很艰难; ( 举例: 一回家就完成作业; 使用安全套; 结 束一段不愉快的关系; 主动约某个人; 与家长谈论某个较为敏感的事情; 当你感情受伤时告诉你的 朋友; 或者是去做艾滋病病毒检测 )

- 写下那个决定是什么, 然后想想让你执行决定变得困难的所有因素, 并将它们写下来;

- 阻碍我们执行一个决定的因素有哪些?

2. 分发 “实施决定的8个步骤” 材料并告诉他们 ( 如果奖学金的例子不太合适的话, 使其他例子替换 它 ) 
- 现在让我们做一个假想的决定;

- 想象你决定要申请去国外大学的奖学金，这是你的梦想。

\section{3. 使用奖学金 (或者其他的) 例子，帮助学生学会材料中的 8 个步骤}

步骤1：怎样才能使执行决定更加容易？（探讨：查出有哪些奖学金是你可以获得的，保持好的成绩， 再次核查申请的指导手册，用电脑或打字机写申请书提交申请。)

步骤2：在执行这个决定时你最有可能遇到的哪些障碍？（探讨：无法查出可申请哪些奖学金, 父母反 对, 申请时的语言障碍, 没有时间好好填写表格, 不敢找老师写推荐信, 成绩不够好, 担心旅费。)

步骤3：你应该怎样私下地、安全地预演你的决定？（探讨：起草一份给老师的请求，练习如何去跟你 的父母谈, 认真阅读奖学金指导手册并想想为什么你是最佳候选人。)

步骤4：与一个支持你的人讨论这个决定和计划。这个人会是谁? (探讨: 朋友, 老师, 亲戚, 父母, 奖学金办公室的人，重视大学教育的人，得到过奖学金的其他人。）

步骤5：考虑如何以最安全的方式执行你的决定。在这个例子中有可能出现哪些风险？（探讨：提出这 个问题可能会导致与父母的严重冲突; 填写申请表时的任何不诚实的地方都有可能导致严重的后果; 到其他国家 (如果奖学金得到了) 可能有的一些风险。)

步骤6：根据你的计划执行决定。实际上你会做些什么?（探讨：与一个支持你的朋友交谈，准备好接 近你的老师, 想想怎样最好地接近可能极不情愿的家长, 努力学习保持良好的成绩一一然后填写申请 表。)

步骤7：如果在执行决定时出现了障碍，制定新的行动计划或者重新考虑你的决定。例如：如果你的父 母不愿意你到其他国家学习，你能做什么?（申请自己国家学校的奖学金。）如果你的成绩没有达到 这个奖学金的要求, 你该怎么办? ( 找寻其他的奖学金的机会。) 
步骤8：注意，即使你没有遇到障碍，你也有权利重新评估你的决定和改变主意。在这个时候，即使没 有遇到障碍, 有什么因素会影响你, 让你重新考虑自己申请奖学金的决定?

( 探讨: 查出本地的大学可以为你提供相同的课程, 决定待在离家近的地方很重要, 害怕只身在外, 上大学时需要做兼职, 决定把计划推迟一年。)

( 你可能想通过说一些话强调教育的重要性, 例如 “如果你们之中的任何人有受教育的机会, 我希望 你们能抓住它”。)

\section{4. 分发 “困难决定的示例”资料并解释}

- 为练习这些步骤, 你们每个人需要从下面的清单中选出一个决定。你们的任务是根据一个做出了该 决定的角色创作出卡通画 (一系列的卡通插图 )，展示这个角色如何通过这8个步骤模式执行一个 困难的决定。有些步骤你们可以很快地完成，例如，这个角色可以仅仅是参照他或她正在做的事。 在你的卡通画中至少有一两个步骤需要有更加详细的东西;

- 你们可以利用这节课剩下的时间开始创作你们的卡通画。接着你们应该至少用 1 个小时的时间完成 它。(注意: 第二个小时可以是课外作业, 也可以是作为第二节课。花点时间保证学生充分理解任 务)

5. 让学生们开始他们的卡通画。教师要在室内到处走动，查看学生们都确实明白了任务，并帮助他们 采用8步骤模式

6. (可作为课外作业来布置) : 给学生更多的时间完成任务。允许学生在画的时候可以相互交流想 法。当所有的画都完成后，考虑将它们挂在墙上或者装订成册 
学生阅读材料

执行困难决定的 8 个步骤

步骤1：考虑怎样才能使执行决定更加容易;

步骤2: 考虑一下在执行这个决定时哪些障碍是你最有可能遇到的;

步骤3：私下地、安全地预演你的决定;

步骤4：与一个支持你的人讨论这个决定和计划;

步骤5：考虑如何以最安全的方式执行你的决定;

步骤6：根据你的计划执行决定;

步骤7: 如果在执行决定时出现了障碍, 制定新的行动计划或者重新考虑你的决定;

步骤8：注意即使你没有遇到障碍，你也有权利重新评估你的决定和改变主意。

困难决定的示例

- 你决定尝试申请去国外上大学的奖学金;

- 你决定反对与父母安排的人结婚;

- 你决定违背父母的意愿与某个人结婚;

- 你决定每一次发生性行为都使用安全套;

- 你决定不与你的对象发生性关系, 即便是他或她以分手威胁你;

- 你决定与你亲密的男朋友 (女朋友) 分手;

- 你决定告诉你的父母 (或者你的伴侣) 你怀孕了; 
- 你决定戒烟（戒酒）;

- 你决定离开你暴力的伴侣;

- 你决定告诉你的父母你是同性恋者;

- 你决定去做艾滋病病毒检测;

- 你决定告诉你的伴侣你做爱时你没有愉悦感或者性高潮;

- 你决定告诉你的配偶、性伴侣或者父母你感染了艾滋病病毒;

- 你决定不按照你们社区里的惯例来做事 (例如, 做礼割、辍学或加入一团伙);

- 你决定告诉你的伴侣或者配偶你知道他或她这段时期与其他人有性关系;

- 你决定告诉你的伴侣或者配偶你有不忠的行为。 


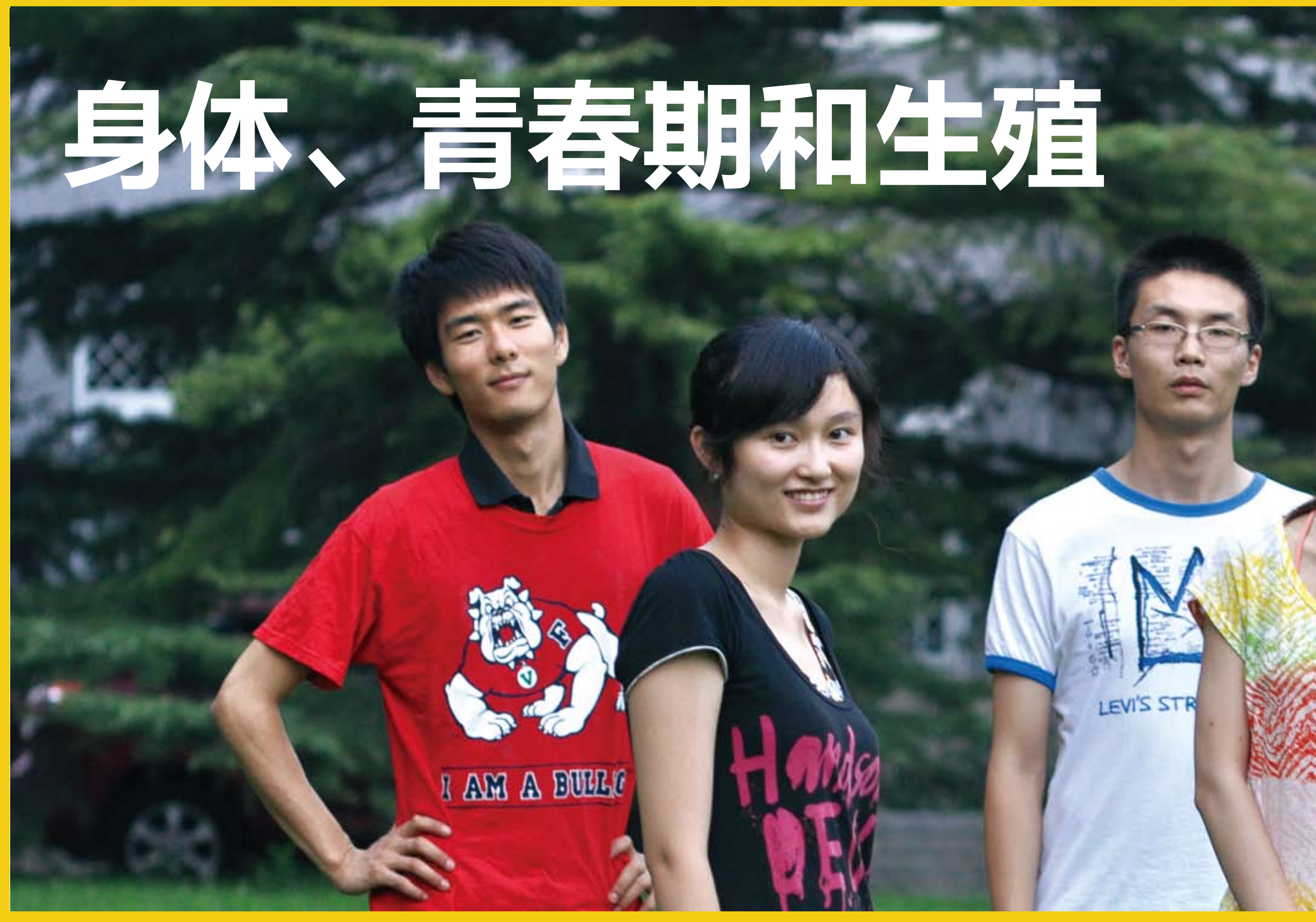




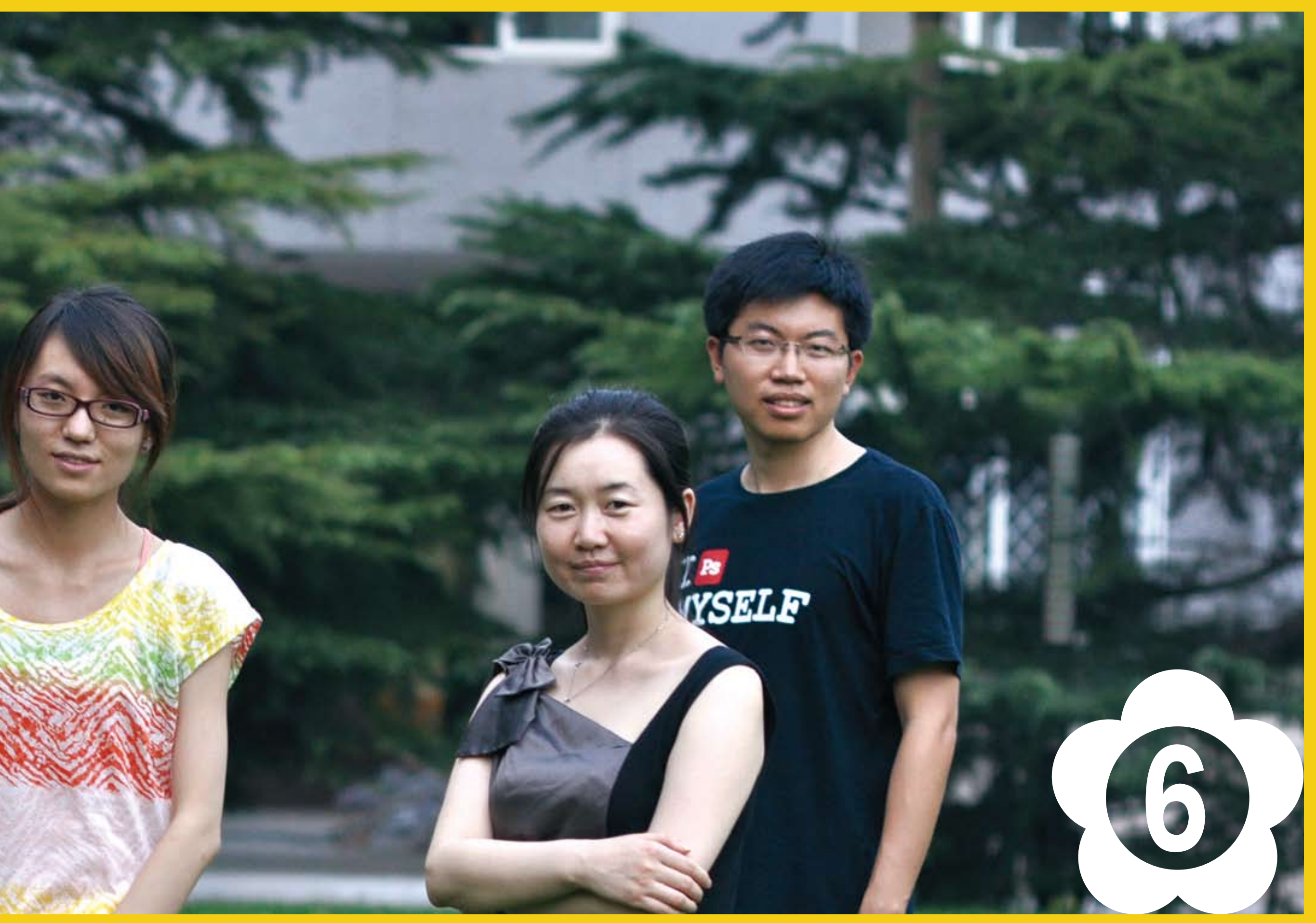




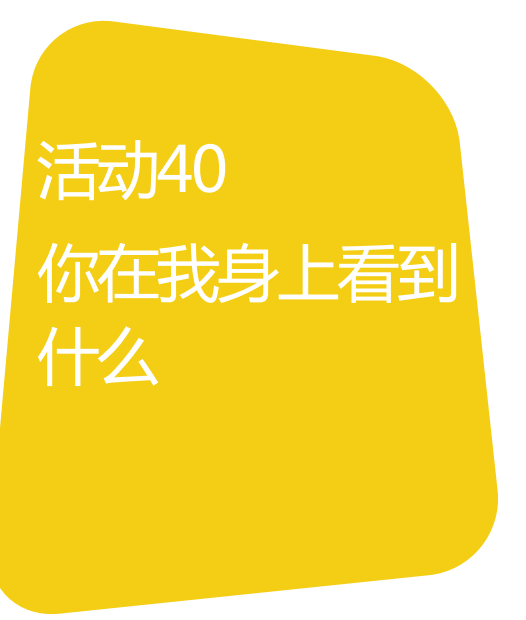

时间：45分钟

材料：黑板、粉笔; 每位学生一张白纸 (最好准备大的彩纸)

准备工作：如果教师了解到有同学之间关系很僵, 在分小组的时候 (第3步 ) 尽量把他们分开。把握 时间很重要（包括在第3步时），这样才能保证在结束前每位同学都有被赞扬的机会。如 果你想进一步了解广告对个人形象的影响的话 (请参见活动9)

概述：学生讨论由于身体发育变化带来的压力, 并写出对自己身体满意的一方面。小组讨论, 每 位学生说出自己一个与外表无关的特点, 而这个特点让别人对他或她产生爱慕

目的：让学生描述外表带来的文化压力如何影响他们的自尊, 增加对自己的优点认识 (包括与外 表有关、无关的 )，并提高他们对身边同伴的关注

\section{1. 通过下列指导性问题进行自由讨论：}

- 许多因素影响我们对自己身体的认识。我们的身体是否强壮健康? 主流社会和媒体是否认同各种类 型的身体都是有吸引力的? 当你的身体在短时间内发生了很大的变化, 你会有什么样的感觉? 是不 是人们对外貌的关注远远大于对其他素质的关注？我们今天将探讨这些问题;

- 试想你现在九岁, 大多数九岁孩子是如何看待自己的身体和长相的? 那时大多数孩子都对自己的外 貌很在乎吗; ( 你可以让学生用1-10来回答上述问题, 1代表完全不在乎, 10代表大多数时候非常 在乎 )

- 当青春期到来时会发生什么? 是不是多数青少年依然不在乎自己的身体和长相? 还是他们开始为自 
己的外貌而担忧了；（表达方法同上）

- 电视和广告宣传的长相、身体该如何? 青少年从广告和电影中收集到什么样的信息和形象? 这种 压力对于谁更大些, 男孩还是女孩;

- 青少年是否会被以貌取人? 大多数人是否希望别人以长相来评判自己? 还有没有什么其他正面的 品质是人们希望被人欣赏的? ( 向女学生也向男学生提问。探讨: 聪明、诚实、幽默、勤劳、勇 敢、善良、有艺术气质、善于音乐或体育、慷慨大方、公平、良好的倾听者、忠诚以及其他此类 性格品质。总结出至少8-10个品质并写在黑板上, 指出女孩和男孩都希望被人称赞这些品质)

2. 让学生拿出纸和笔, 告诉他们:

- 想一想自己相貌和身体中你满意的地方, 可以是你的笑容, 你的眼睛, 你走路的姿势, 你的肌 肉, 你的头发甚至是你的身高。也可以是你的体形, 你的鼻子, 你的舞姿, 你的脸型, 你的胳膊 和腿, 你的手, 你的皮肤, 你的酒窝或者你的嘴唇。也可以是其他;

写下一句诗意的话来描述你满意的一点, 只需写给自己, 不需要跟其他人分享 ( 例如: 我的笑容 照亮了整个房间; 我的眼神深遂如大海）;

- 10 分钟时间完成这个任务，并收好自己所写的东西。

3. 在学生收藏好自己所写的纸片后, 把学生们分成 5 人一组, 给每组 5 张表和一支马克笔, 解释说:

- 请记住我们都希望得到除了长相以外其他方面的赞扬;

- 从你这组的某个人开始, 一个人记下发言人的名字, 然后, 小组内轮流开始发言说出关于这个人 一些与相表无关的让人喜欢的地方。可以是你之前提到过的 (参见黑板) 或其他正面的优点。这 可以是你一眼就可以发现的, 也可以考虑是你以前不曾注意的某个人的某个方面;

- 当你说出这些优点时, 把这些优点记在他名字的下方; 
- 当你记下这些优点之后, 把这张纸交给下一个人。继续这个过程直到这张纸传递了一圈;

- 要确保你在表扬别人的时候既用口头说出来, 同时也写了下来。花几分钟, 每个人的纸都这么传递 一圈。老师会帮助学生计时, 使每个人都能轮到; ( 把余下的时间平分成5份。教师在教室走动, 确保各组能按时保质保量完成练习 )

在活动开始之前, 哪些是我们需要制定一些游戏规则呢; （探讨：需要对每个人保持尊重, 想一些 新的评价而不是重复前面的, 挨个轮流。即使你们组有一些你不是很喜欢的人, 记住一个道理, 每 个人都有自己发光点, 你希望别人怎么待你, 你也应该怎么待人。

当活动结束时, 你可以保留那张写满自己优点的纸。这是你赢得的! 


\section{时间：60分钟}

材料：准备美工用具, 如纸张、马克笔; 海报板或大张纸; 大头针、针、订书机等 (关于男 孩和女孩青春期可靠的参表，比如《指导手册》中的对应章节。也可登陆网址：www. popcouncil.org/publications/books/2010_ItsAllOne.asp )

准备工作：事先温习或熟悉事实参表中的内容, 以保证在学生的制作过程中可以补充或者加以纠正。 复印青春期男孩女孩情况一览表。如果可行, 安排学生们给未进入青春期的学生们展示他 们的创作成果

概述: 学生创作书籍和歌曲, 来解释青春期的身体变化

目的: 让学生描述青春期的基本生理变化; 增强团队精神和表达能力

1. 告诉学生这个活动是关于青春期时的身体变化的，可以提问

- 什么年龄阶段去了解青春期给我们身体带来的影响是最合适的, 是在青春期的时候还是青春期之 前。是不是大多数孩子对青春期的基本知识都有足够的了解;

- 你需要给即将进入青春期的青少年制作关于青春期的材料。每三人一组。每组要么全是男孩, 要 么全是女孩。女孩组展示女孩的青春期材料, 男孩组展示男孩的青春期材料;

- 可以用任何方式展示你们的材料。例如：你可以把这些材料制作成流行歌曲歌词、儿童书籍、海 报，或者你们也可以在报纸上开办针对青春期的孩子 “亲爱的医生” 专栏，并对来信进行回复。 
尽量发挥你的创造力! 但切记, 你所提供的信息必须准确而且有帮助; ( 安排好以后, 请向学生 们解释说他们的成果将展示给一群比他们年龄稍小的学生们。)

- 为了帮助学生, 老师给每组分发一个青春期男孩女孩知识事实说明表。

2. 三人一组，并分给他们材料。把美工材料准备好。告诉他们每小组有25分钟去完成上述工作

3. 如果你计划把这些材料展示给年龄稍小的孩子们, 请利用余下的时间, 教师在课堂转一下, 在展示 前，先与每组商讨他们的成果。帮忙改正或者填充不正确或不完整的信息

如果你只是计划在本班同学中展示这些材料: 请各组在时间允许的情况下展示他们的作品,来不及 展示的下节课再展示,让其他学生也参加对展示者指正和补充。

4. 在这个活动的最后，把其余那部分青春期男孩女孩知识事实说明表发下去，让每个人都可以有一份 男孩的材料和一份女孩的材料 
时间：45分钟

材料：黑板、粉笔、纸条、给教师的答案（“青春期发生的变化”）

准备工作：把 “青春期发生的变化” 中的每一条抄写在一个纸条上，分别折起来分别放入一个信封。 在进行步骤3时根据你所在的环境, 审阅修改或补充青少年进入青春期时生活变化的情况

。学生分析男孩和女孩在青春期期经历的社会变化 ( 注: 在做完这个活动之前, 学生应该完 成活动 41 及其他与青春期发生的生理变化相关的其他活动 )

让学生说出在青春期时性别角色发生的三种变化, 并批判式地评价这些变化在女孩, 男孩 以及男女关系上所产生的影响;增强批判性思维能力

1. 介绍并解释这些问题:

- 所有的青少年在青春期都会经历一些变化, 其中的一些变化是正常的生理发育, 还有一些变化并非 生理上的。它们是人们对待你的方式的变化;

- 老师会在教室里走动, 让一名学生抽出一张老师手里的纸条, 大声的念出纸条上的内容, 看这个变 化是青春期世界上所有青少年都发生的生理变化, 还是在进入青春期阶段后社会对青少年的看法发 生的改变; (如果没有准备这些纸条, 可以随机挑选 “青春期发生的变化” 这张表上的内容, 念出 并让学生们评判 )

- 剩下的时间详细解释青少年在青春期所受待遇变化, 老师不需再过多地解释身体变化。 
2. 在黑板上方写下 “人们对待你的方式的变化”，然后把黑板分为两块，一块注明是 “男孩”，一块 注明是 “女孩”。并解释:

- 年轻人在青春期会发生什么变化? 人们对待他们的方式是否有所改变? 当我们更详细地考虑这些 问题时, 请首先分辨一下它们是针对男孩还是女孩, 如果这个变化对男孩、女孩都有影响, 那么 它对男孩与女孩的影响是否相同。

3. 读出下一页单子上的每一条内容 (在教师审阅和修改之后)，然后问学生这些内容是针对男孩还是 女孩, 或者两者都影响。如果有争议, 大家讨论, 并把这点填到相应的变化下面

青春期：人们对待你的方式的可能变化

- 更自由的出入公共场所（街道，公园，社区中心，购物区）;

- 更少的自由出入公共场所;

- 更多的家务责任（如做家务或照看儿童）；

- 更多的责任开始去挣钱;

- 在着装和穿衣哪里该遮, 哪里该露方面有更大的压力;

- 男孩和女孩的社会接触可能会更多或者更少;

- 面临一些很危险的成人仪式（如女性的割礼）;

- 面临一些无害的成人仪式;

- 面临获得性经历的社会压力;

- 面临准备结婚的社会压力;

- 更多机会成为学校和社区的领导者; 
面临在运动上获得成功的压力;

面临更大的中途辍学的可能;

遭遇性骚扰;

- 加入社会帮派的压力;

面临为了遵守社会性别而必须冒险的压力;

面临用性来交换礼物, 金钱和学费的压力。

4. 把相同性别的学生分为一组，请他们讨论青少年在青春期的社会性别角色和生活是如何变化的，特 别要问如下问题:

- 青少年在青春期所遇到的社会期望和经历的变化, 是巨大的还是微小的变化;

- 你注意到男孩在青春期会遇到什么变化? 总体上来讲, 他们的自由空间是扩大还是缩小? 这些影响 在青春期后是变得无足轻重了? 还是会继续影响到其成人阶段;

- 你注意到女孩在青春期会遇到什么变化? 总体上来讲, 他们的自由空间是扩大还是缩小? 这些影响 在青春期后会消失还是会继续影响到其成人阶段。

5. 请各组重新集合，问如下问题：

- 有没有可能发生其他的变化呢? 是不是有可能使我们生活的更好, 更公平一些呢? 你希望发生什么样的变化呢。

说出一种方法, 可以使社会让年轻人更好地度过青春期。 
说明：把下面的每一条抄在一个小纸条上, 折起来分别放入一个信封。如果需要的话, 以下清单 可以作为解答学生疑问的答案, 确保学生们正确辨别哪些是青春期的生理变化 (身体上的), 哪 些是社会变化 ( 基于性别的不同等对待 )。

\section{生理变化：}

- 体毛生长;

更易流汗;

乳房发育 (女孩);

遗精 (男孩);

变声 (男孩);

全面成长, 需要额外营养;

- 性需求增加;

○经, 分泌粘液 (女孩)。

\section{人们对待你的变化：}

- 更多机会成为学校和社区的领导者;

成人仪式;

- 各种责任的改变;

- 关于性行为的压力;

- 有关婚姻的压力;

- 关于着装的新规定;

- 关于男孩女孩如何相处的新规定;

- 自由许可的变化。 
时间：步骤1-2, 20分钟 步骤3-5, 25分钟

材料 每位学生一张字谜表、有趣事实表及给老师的答案; 关于生殖健康的可靠信息来源（比如 与本书配套书《的指导手册》第6单元以及单元中的事实说明表 )

准备工作：认真复习材料，特别是月经周期、男性和女性的生殖系统、生育、怀孕、不孕不育及辅助 生育等部分。决定是否将所有的字谜表的答案都放在发放的材料里。根据时间和内容, 决 定保留有趣的事实表的所有内容还是删除一部分

概述：根据一个字谜和一个练习, 学生们学习关于身体和生殖方面的一些有趣的事实 (注意: 这 个活动只是对关于生殖这个主题的讲座或读物的一个补充, 而不会全面介绍这个主题 )

目的：扩大学生关于生殖健康方面的知识面, 增加他们对于生殖生物学方面的兴趣

\section{1. 把字迷发给学生们并作如下的解释}

我们已经学习了人类生殖的基本步骤, 作为复习, 根据每个暗示, 将正确的答案填入字谜中;

答案的每个字母都必须填入方框, 既不能多也不能少;

横行和竖行重合部分是两个词中相同的字母。

2. 过10-15分钟之后, 教师在教室走动看看同学们是否正确填写出答案

3. 分发 “有趣的事实” 表, 并解释: 
我们现在需要了解更多关于人体的事实, 它们中的大多数是有关生殖的, 有些是人体有趣的事实;

每个人花 5 分钟时间阅读关于人体有趣的事实, 然后找出你觉得最有趣的一个事实;

与你身边的同学交流最有趣的事实, 看看是否相同;

你们中的多少人对最有趣的事实有着相同的选择? 不同选择的有多少人; (举手表示)

你们中的多少人从这个表格中学习了至少一条新的知识。

4. 请学生自愿说出哪个事实他们觉得最有趣, 请根据教师答案手那提供一些补充事实, 尽量用问题去 引导学生说出新的知识

5. 如果时间允许的话: 当你们说到了第十五条事实的时候，让学生两人为一组，相互帮助用臂展比较 身高。解释一下这个和生殖健康并没有直接关系, 但这也是对我们自己身体认识的一个方面

学生讲义:

生殖健康系统字谜：

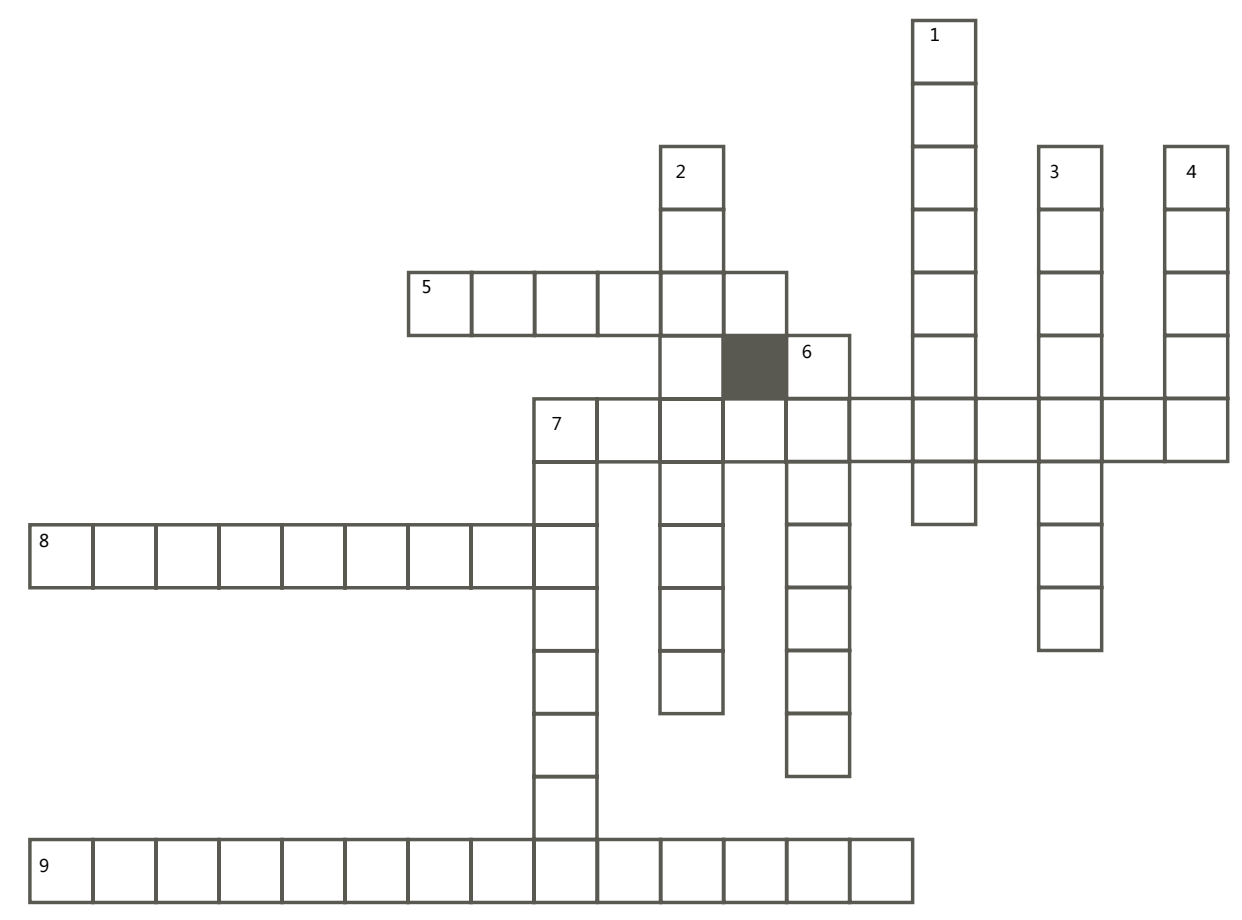




\section{竖向}

1.睡眠时流出精子（两个词）

2.从一个卵巢排出一个卵子

3.精子产生之处

4.精子从阴茎中排出粘液

6. 从孩子发育到成人并且能生殖的快速成长过程

7. 阴茎勃起

竖向：

1. 梦遗

2. 排卵

3. 睪丸

4. 精液

6. 青春期

7. 勃起
5. 婴儿发育的地方

7. 精子从阴茎有力的射出

8. 妇女断经的时候

9. 连接卵巢与子宫的导管, 受精在此发生

( 两个词 )
横向：

5. 子宫

7. 射出

8. 绝经期

9. 输卵管 
( 改编自生殖健康研究所：我正在变化的身体 <www.irh.org > )

\section{关于身体的奇特事实}

在这里面, 你觉得哪一项最神奇最有趣。读下面神奇事实, 圈出你认为最值得了解的:

很多女性发现她们在排卵期性欲较强;

- 不育夫妇不孕症的原因发生在男性和女性的比例是几乎相同的;

- 人体内的小肠长度大约有7米长;

- 精子在睪丸内发育, ( 睪丸在阴囊中) 精子正常发育需要比体温稍低的温度, 因为睪丸在体外, 能 保持较低温;

- 因为女性每个月排卵期只有几天, 女性有可能怀孕的时间每个月也只有几天, 但男性从青春期开始 就一直有生育能力;

一次射精中的精子数量能达到上千万;

人体一天正常产生一升唾液;

一个女性可以从她阴道分泌的粘液分辨出什么时候排卵, 从而推断出什么时候更容易受孕, 同样也 可以预测出月经周期;

- 人体中超过 $50 \%$ 是水;

孕妇肚子的形状和孩子的性别没有什么关系;

在怀孕晚期, 女人的髋关节变宽, 使盆骨扩大, 便于生产; 
在极少数情况下, 女人产生两个卵子。如果两个都受精, 那么会出现异卵双生; 如果一个受精卵 在卵裂时一分为二, 就出现同卵双生;

大多数男孩在青春期都会有梦遗现象;

胎儿性别是由精子 ( 来自父亲) 而非卵子 ( 来自母亲) 决定的;

人们张开双臂的长度等于整个身体的高度。

\begin{tabular}{|c|c|c|}
\hline & 事实 & 补充信息 \\
\hline 1 & 很多女性发现她们在排卵期性欲较强。 & $\begin{array}{l}\text { 这有助于确保性爱时间发生在最有利于物种延续 } \\
\text { 的时期。(可提到进化论) }\end{array}$ \\
\hline 2 & $\begin{array}{l}\text { 不孕症的原因发生在男性和女性的比例是几 } \\
\text { 乎相同的。 }\end{array}$ & $\begin{array}{l}\text { 在有些地方, 不孕常常怪女方有问题, 因为男人 } \\
\text { 不知问题出现在他们身上。要明白不孕的真正原 } \\
\text { 因往往是困难的 (详见不孕不育表 ) }\end{array}$ \\
\hline 3 & 人体内的小肠长度大约有7米长。 & $\begin{array}{l}\text { 在体内, 小肠是一圈圈卷起 } \\
\text { 的, 只占很小的地方。 }\end{array}$ \\
\hline 4 & $\begin{array}{l}\text { 精子在睪丸内发育 ( 睪丸在因囊中 ), 精子 } \\
\text { 正常发育需要比体温稍低的温度, 因为睪丸 } \\
\text { 在体外, 能保持较低温。 }\end{array}$ & $\begin{array}{l}\text { 在持续高温的环境下（比如靠近焚烧炉工作, } \\
\text { 穿过过紧的裤子和内裤等都会增加阴囊睪丸的 } \\
\text { 体温）。（详见男性性与生殖系统表） }\end{array}$ \\
\hline 5 & $\begin{array}{l}\text { 女性能有可能怀孕的时间每个月也只有几 } \\
\text { 天, 但男性从青春期开始就一直有生育能 } \\
\text { 力。 }\end{array}$ & 随着年龄的增长, 生育能力逐渐降低 \\
\hline 6 & 一次射精中的精子数量能达到上千万。 & $\begin{array}{l}\text { 睪丸内精子成熟时间约75天。（详见男性性 } \\
\text { 与生殖系统表 ) }\end{array}$ \\
\hline
\end{tabular}




\begin{tabular}{|c|c|c|}
\hline 7 & 人体一天正常产生一升唾液。 & $\begin{array}{l}\text { 除了水, 唾液还含有其他一些化学物质, 例 } \\
\text { 如分解食物的酶。 }\end{array}$ \\
\hline 8 & $\begin{array}{l}\text { 一个女性可以从她阴道分泌的粘液分辨 } \\
\text { 出什么时候排卵, 从而推断出什么时候 } \\
\text { 更容易受孕, 同样也可以预测出什么时 } \\
\text { 候月经期该来了。 }\end{array}$ & $\begin{array}{l}\text { 如果一个女性知道她什么时候排卵, 她可以 } \\
\text { 利用这个消息避孕或者尝试怀孕。（详见月 } \\
\text { 经周期表 ) }\end{array}$ \\
\hline 9 & 人体中超过 $50 \%$ 是水。 & 人最大的器官是皮肤。 \\
\hline 10 & $\begin{array}{l}\text { 孕妇肚子的形状和孩子的性别没 } \\
\text { 有什么关系。 }\end{array}$ & $\begin{array}{l}\text { 对孕妇的研究发现孕妇对子形状 } \\
\text { 与孩子性别无关。 }\end{array}$ \\
\hline 11 & $\begin{array}{l}\text { 在怀孕晚期, 女人的髋关节变 } \\
\text { 宽, 使盆骨扩大, 便于生产。 }\end{array}$ & $\begin{array}{l}\text { 女人在快生育时, 行动一定要小心, 因为松 } \\
\text { 动的关节会让她觉得重心不稳。 }\end{array}$ \\
\hline 12 & $\begin{array}{l}\text { 在极少数情况下, 女人产生两个卵子, 如 } \\
\text { 果两个都受精, 那么会出现异卵双生; 如 } \\
\text { 果一个受精卵在卵裂时一分为二, 就出现 } \\
\text { 同卵双生。 }\end{array}$ & $\begin{array}{l}\text { 异卵双胞胎的基因就像兄弟一样, 而同卵双 } \\
\text { 胞胎来自同一个细胞核, 他们的基因是完全 } \\
\text { 相同的。（详见生殖与怀孕表） }\end{array}$ \\
\hline 13 & 大多数男孩在青春期都会有梦遗现象。 & $\begin{array}{l}\text { 梦遗 (也叫遗精 ) 一般是男孩或男人在睡觉 } \\
\text { 的时候射放的, 是一件很正常的事情, 对身 } \\
\text { 体没有伤害。 }\end{array}$ \\
\hline 14 & $\begin{array}{l}\text { 胎儿性别是由精子 ( 来自父亲) 而非卵子 } \\
\text { ( 来自母亲) 决定的。 }\end{array}$ & $\begin{array}{l}\text { 在一些地区, 妇女有要生男孩的文化压力。实 } \\
\text { 际上, 孩子的性别是由男性决定的。如果胚胎 } \\
\text { 是由母亲的卵子X染色体和父亲精子的一个X染 } \\
\text { 色体或Y染色体组成。如果精子有X染色体, } \\
\text { 胚胎有XX染色体而为女性。如果精子有Y染色 } \\
\text { 体, 那么胚胎就有XY染色体而为男性。（详见 } \\
\text { 生殖与怀孕表） }\end{array}$ \\
\hline 15 & 人们的展开双臂的长度等于整个身体的高度。 & 试试看! \\
\hline
\end{tabular}


时间：步骤1-6: 45分钟 步骤7（作业），40分钟 步骤8-11：40分钟

材料 每位学生一份课堂练习材料。提供给选择A-F话题的学生的材料内容包括: 本卷配套书 《指导手册》上的性选择表、不孕不育表、辅助生育表、生育和母乳喂养表及流产表 (也 可登陆网站<http://www.popcouncil.org/publications/books/2010_ItsAllOne.asp> )

准备工作: 认真复习所讲内容材料并在需要时做出修改。复印课堂练习材料, 或将最终选出的话题列 表, 在课前抄在黑板上。帮助学生寻找传播研究成果的方式 (比如当地报纸, 可作为电台 一分钟短评系列) 或者学校集会, 或者一本书的形式等

概述：学生了解15个关于当代生殖的公共卫生政策 (从早育到性选择)。他们在一篇杂志中写下 自己的个人观点, 用来为公共教育概括、编辑成一个简单的新闻故事

目的：增加学生对生殖方面的知识以及对生殖方面公共卫生政策的关注。增强他们的写作和编辑 能力；坚定他们在社会中表达自己的信念

1. 解释

我们已经了解了人类生殖。生殖总是个人问题吗? 只与当事人有利害关系吗? 生殖的某些方面是 否涉及到社会? 比如说健康部门、社区、或者其他; (探讨例子, 比如健康服务资金、有关流产的 法律、医院的规定以及类似的例子)

事实上, 生殖健康是一个很重要的社会问题, 有时它又是争议的来源。在现代社会, 伴随着新的 技术、艾滋病和不断变化的文化道德观，有关生殖的问题与人们生活息息相关，它们已经成为人 们需要掌握的重要知识 


\section{6}

今天我们将启动一个课题, 你们每个人都可以选择一个自己感兴趣或者对你有意义的话题进行探 索, 我们课题最终成果会在社会上展示。（解释怎么做一一例如：在当地报纸上发表文章）

2. 汶览话题列表, 让同学们自愿提出简短的定义和解释, 这样可以确保每个人对话题有基本的理解。 然后解释:

- 每个人都要找到对自己有意义的话题, 或对你重要、你感兴趣的话题。这不是一个大的调研课题, 内容不需要超过两段, 每一段需要有简练、引导性的问题。而且这些段落应该是整个成果的浓缩与 精华;

- 今天你要选定话题, 对它进行初步了解。对一些话题, 我会提供一些参考资料, 对于其他的话题, 你需要在班里采访三位同学并写下他们的想法。

3. 阅读学生讲义中关于课外作业的步骤。如果学生材料不够, 将 “特别提示” 的说明部分抄到黑板上

4. (如果你把选择性的编辑任务包括在内) : 明天你会和另外一个同学交换你的第二个段落 (一页新闻剪 辑），你们一起对段落进行编辑，确保语句清晰、语法正确、逻辑严谨并且有趣。你们将这些新闻剪 辑整理成一篇关于 “当代怀孕和生育问题” 的文章发表在..... ( 例如，一个当地报纸或广播台 )

5. 确保任务清晰。让学生在一张纸上填写自己选择的话题，如果他们有任何问题，鼓励他们和你交 流，尽量避免话题的重复

6. 用预留剩余的时间让学生们开始

- 对于选择A一F话题的学生: 给他们阅读材料让他们阅读（如果有书面材料可以让学生带走, 他们 可以在家里完成阅读 )

- 对于选择G一N话题的学生: 让他们互相采访并做笔记。如果可以, 鼓励他们寻找不同的观点（采 访也可以在家中完成，例如，向家庭成员采访）

7. 留下五分钟解释课外作业 
选择性活动：编辑、分享、出版

8. 让学生结对编辑他们的第二个段落。如果两个或更多的学生选择同一个题目，把他们分为一组并鼓 励他们把他们的文章整理成一篇文稿，这样读者就不会感到困惑和无聊了

9. 问学生:

- 好文章的特点是什么; ( 探讨: 主题明确、避免重复、结构清晰、用词汇恰当、适当的感情、合 适的语法、准确的拼写。把这些写在黑板上 )

有什么方式对其他人的文章反馈; ( 探讨: 提及哪些积极方面是你喜欢的; 提出批评时保证谨慎 和尊重的态度; 提供一些意见, 但不要改写他的作品 )

什么方式能反馈其他人的文章。（探讨：感谢别人的好建议; 承认你哪里遇到了困难或需要帮 助; 再次尝试之后请给更多的反馈 )

10. 让每位学生互相提供意见反馈，给第一位和第二位各10分钟时间向对方提供反馈，并各给他们10分 钟作修改。每次10分钟结束时, 都要宣布并提醒

11. 收集修改完的文段(和个人陈述)，将每个新闻摘要贴在墙上，让整个小组阅读

如果时间允许 (或者第二天)，让一些学生分享他们的个人答复或者他们最终的新闻文章

理想情况下: 鼓励学生编辑他们的新闻摘要, 让他们将摘要放在一起或者编辑成一个单一的数码文 件, 并发表在报纸上或者通过其他方式来宣传。鼓励学生在寄出最终成果前, 运用自己的创造力去起 题目、写信来包装成果。

\section{课堂练习材料}


关于话题的新闻说明：请在不同两张纸上分别写两段。第一段是个人回答, 你仅仅需要写下你对话题 的感受, 也可以写下任何有关自己的经历。第二段是新闻摘要, 它的目的是教育其他人。

\section{准备摘要:}

1. 收集关于话题的信息。对于A-F话题, 可从给定资料中找出信息; 对于G-N话题, 可从简短的采访 中收集信息, 采访对象可以是你的同学, 但尽可能寻找不同的观点。

2. 解释话题是什么。

3. 描述为什么这是新闻或者为什么这个话题是争议性的或是焦点。

4. 用一个结论或者一个值得读者思考的问题结束。当你写这篇文段时, 要明白读者是大众, 例如报纸 读者或广播听众。

5. 最后末尾，注明你是通过阅读材料还是采访他人收集的信息。

怀孕和出生 : 当代话题和引导性问题

阅读并思考如下话题 ( A--F )
A. 重男轻女地区的性别选择
见: 性别选择事实说明表
思考: 我们如何从长远的角度解决这一问题。
B. 辅助生育 (用来为不孕夫妇服务的科技)
见: 不孕和辅助生育表 
思考：你对花钱雇人帮忙生孩子（代孕母亲）怎么看。

C. 剖腹产: 有些时候在不需要剖腹产的时候, 我们做了这个手术; 而有时, 当真的需要剖腹产时, 却 无法获得这个手术。怎么做才是正确的?

见: 分婏及母乳哺育表（剖腹产部分）

思考: 经济因素是怎样影响剖腹产率的?

D. 当流产不是一个选择时：被迫流产或被迫生育。

见: 流产表

见: 第七单元意外怀孕和流产部分

思考: 是不是任何人都应被迫流产? 是不是任何非意愿怀孕的人都应被迫生下孩子?

E. 产科瘘

见: 产科及母乳哺育表 (产科瘘部分 )

思考：为什么许多人不懂得这个问题? 我们怎么去解决它。

F. 怀孕及艾滋病病毒检测呈阳性

见: 分娩及母乳哺育表 (艾滋病病毒呈阳性和怀孕部分)

见: 生殖怀孕表 ( 提倡健康怀孕部分 )

思考：你对这个问题怎么看。

采访并思考话题 ( G--O)

G. 少年父亲 
采访3个人, 问年轻男孩: 是否准备好做父亲了? 大多数少年父亲能对他们子女负责吗?男孩在避免意 外怀孕这个问题上应该承担怎么样的责任?

H. 女孩如何对待经期

采访三个女孩, 问: 当经期到来时, 女孩一般如何保持卫生? 用什么吸收流出的血? 痛经会不会成为 经期的一个问题?

I. 领养：该不该知道你的亲生父母/孩子

很多孩子是被领养的, 领养人并不知道领养孩子的生父生母是谁。有时, 孩子会得到一些他们自己生 父生母或其中一人的信息，甚至和他的生父或生母取得了联系，但有时既无信息也不可能取得联系。 采访三个人: 问: 你觉得哪样最好? 孩子应该知道自己生父母的一些信息吗? 孩子有机会跟他或她生 父母取得联系吗?

J. 产前培训班: 为准爸爸和准妈妈做好生育和养育孩子的准备

在一些地方, 准爸爸和准妈妈会一同在生孩子之前参加一个培训班, 学习生育和育婴的常识。

采访三个人, 问: 我们的社会中需要这种培训班吗? 如果父亲了解这方面的信息与否有什么不同吗?

K. 分婏时父亲是否应该在现场

采访三个人。问: 孩子分婏时父亲是不是应该在产房中? 这会不会对父亲跟孩子的情感有什么影响?

L. 产假及丈夫陪产假政策

采访三个人。问: 你觉得生完孩子后产妇应该休息多久? 在有些国家, 父亲也会有陪产假, 你觉得这 个主意怎么样? 你觉得父亲的陪产假应该有多久?

M. 谁教给你生殖知识? 在什么时候? 
采访三个人。问: 谁告诉你婴孩是从哪里来的? 当时你几岁? 你觉得学习人类再生殖的最好途径是 什么?

N. 政府奖励生育的政策影响家庭大小

有些政府为了增加人口, 奖励多生孩子的家庭, 有些政府担心人口增长过快, 奖励终止妊娠的家庭。

采访三个人。问: 你赞成这两种政策么? 为什么?

O. 上学时期怀孕

每天都有在学的少女怀孕, 在有些地方她们被允许继续上学, 但有些地方她们将被迫离开学校。

采访三个人, 问: 使这些女孩和他们的孩子得到最大的保护的最好政策是什么样的? 孩子的父亲在这

个时候应该起什么作用, 担当什么责任? 


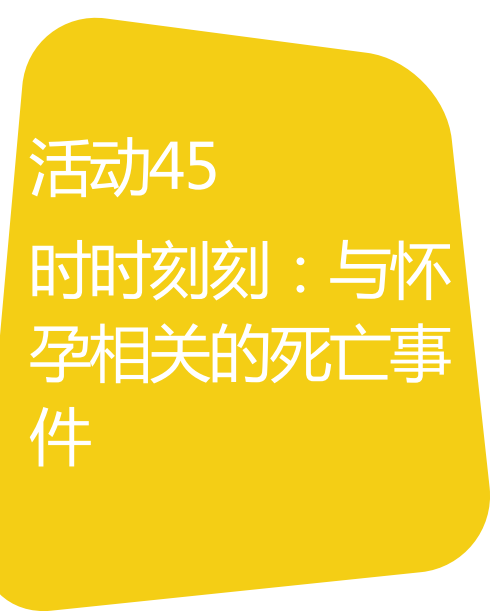

时间：60-85分钟, 取决于使用案例的个数

材料：黑板、粉笔; 复印每份案例

准备工作：阅读8个案例研究, 修改或替换案例, 以使它们适合当地社会环境并且真实。汶览相 关内容 (本卷配套书《指导手册》中第六单元中关于 “怀孕中” 的内容, 以及关于生 殖、怀孕、生产、母乳喂养的相关资料 )，可登陆<www.popcouncil.org/publications/ books/2010_ItsAllOne.asp>查找相关资料和信息

概述：学生阅读关于产妇死亡的案例研究。通过思考和讨论, 探索如何阻止这些死亡

目的：使学生能够描述出贫穷和性别不平等导致孕妇死亡, 或导致严重健康问题的主要途径; 探 讨可以如何避免这些结果; 提高他们对产妇死亡这一问题的关注度; 增强他们的分析能力

1. 将 “产妇死亡”这一词语写在黑板上。提出以下问题, 并在黑板上写出答案

- 你认为 “产妇死亡” 是什么概念, ( 探讨得出一个类似于这样的定义: “怀孕和生产所导致的妇女 和女孩死亡” )

- 这里有些疑问: 你认为世界各地妇女和女孩因怀孕而死亡的比率有多高? ( 允许进行猜测 ) 每周一 人? 每十分钟一人? 每天一人? (答案是每年的每一天的每一分钟都有死亡发生 ) 在我们学习与 “产妇死亡” 相关的知识的这段时间里, 六十位妇女和女孩会由于这一原因而死去, 这相当于每年 50 万的死亡数。更多的女性虽没有死亡，但却被怀孕带来的疾病所困扰; 
- 这些死亡事件是在在全球分布平均吗? 比如说, 你猜测在发展中国家（不那么工业化和富有的 国家）的孕妇死亡率是百分之多少? (允许猜测) 十分之一? 一半? 三分之二? (答案是99\%) “产妇死亡” 在发达国家中极为苹见;

- 年轻女孩承受着更大的风险。与成年妇女和女孩相比, 十五岁以下的女孩在分婏时有更大的死亡 危险;

- 你认为多少 “产妇死亡” 是可以避免的? (允许猜测) 一小部分? 一大半? 三分之一? 专家指 出, 几乎所有这类死亡都可以避免;

- 你认为现在的妇女和女孩跟几十年前相比, 更容易还是更不容易发生 “产妇死亡”（允许猜测） (答案是, 几乎没有差别)

- 今天我们将学习更多相关内容, 你将明白你可以如何帮助自己及身边的人改变命运。

2. 根据你使用的案例研究数量, 将班级分成相应数量的小组, 发给每个组一份案例来阅读。先将它折 起来, 学生暂不阅读

解释:

- 每一组都拿到了一份不同的关于 “产妇死亡” 的案例研究。阅读后请在组内讨论: 哪儿出了问 题?（什么是造成或导致那些妇女和女孩死亡的主要原因？）应该怎么做？（应该怎么做能让结 果不同, 从而拯救她的生命? ) (在黑板上写下这些问题 )

学生将以一个短剧 (2-3分钟) 的形式表演这个妇女的故事。首先要按照案例中所写来表演, 然后 加入你认为能够挽救那个妇女生命的一些方法再来表演。可以让同一批人演两遍, 也可以让同组 的其他人来演第二场。 


\section{4. 在短剧后留出15分钟来讨论}

你对这些故事有何感觉? 是案例中的哪一方面让你产生了这种感觉;

个人、家庭成员、社区、医疗服务人员以及政府都可以采取措施阻止这种死亡的发生。在一个妇女 怀孕之前, 需要做哪些重要的事情来保障她们的（和她们的新生婴儿的）健康呢? （将学生的回答 写在黑板上; 可以给些提示)

- 在怀孕期间能做什么; ( 将学生的回答写在黑板上; 可以给些提示 )

- 在分婏时能做什么; ( 将学生的回答写在黑板上; 可以给些提示)

- 我们怎么来预防15岁以下女孩的“产妇死亡”；

- 你将在以后怎样运用今天学到的知识?

\section{( 学生阅读材料 产妇死亡的案例研究 )}

瑞妮的故事: 当瑞妮13岁的时候, 她的母亲死于一次分婏。她的父亲告诉她, 她不得不辍学为家里做 饭并照顾年幼的弟弟妹妹。她还在上学的哥哥试图帮助瑞妮继续她的学业, 但是瑞妮因为还有其他的 事情要做而无既学习, 不久就放弃了。在十九岁的时候, 瑞妮嫁给了拉马什并与他和他的父母住在了 一起。拉马什的家人挺看重瑞妮的，因为她懂得照顾孩子，就希望她尽早生个孩子。

当瑞妮怀孕的时候, 他的丈夫不断地说她该生个儿子。尽管如此, 瑞妮还是偷偷想着想要一个女儿, 她默默许诺, 因为自己没有念完书, 如果她有了女儿她一定让她完成学业。在怀胎八个月以后, 根据 当地的习俗, 瑞妮回到了自己家乡生孩子。当她临盆时, 请来了当地的医生, 一天一夜后孩子还没有 生出来, 医生才向她推荐最近的医院, 大约有一公里远。因为天色已晚, 直到第二天早上才有人将瑞 
妮送到医院, 瑞妮最终到医院时已筋疲力尽, 她生下了孩子。尽管她的丈夫看到这个刚出生的婴儿不 是一个儿子时有些失望, 但是瑞妮仍对她新生的女儿默默地念着自己许下的诺言, 与此同时, 她开始 大量流血, 尽管工作人员马上给她输血, 但瑞妮还是昏迷了过去。医院的人员竭尽全力, 可是瑞妮还 是因为失血过多, 离开了人世, 就像当年她母亲一样。

小慈的故事: 小慈是个15岁的女孩, 她的时间用在学校、家里学习和做家务上。她曾和一个在学校 偶遇的男人出去过几次。他送给了她一些礼物和一些钱, 她用这钱付了书费, 然后小慈和他发生了性 行为。她要求这个男人带安全套, 这是她在学校学到的, 但是这个男人告诉她不必担心。她其实并不 是非常喜欢他, 所以她决定不再与他见面。但是很快, 小慈意识到自己怀孕了, 当她把这件事情告诉 她的父母的时候, 她的父亲打了她, 但并没有将她逐出家门。几个月之后她辍学了, 住在另一个镇上 的姑姑家里, 她的父母同意姑姑来养这个孩子, 这样就不会有人知道小慈怀孕的事情。当她临盆时, 她的姑姑告诉她要挺住, 孩子就会生出来了。但是, 这个婴儿卡在了产道里, 姑姑叫了当地的接生 婆, 她用刀切开小慈的阴道口, 使之更宽些, 这样婴儿很快就生出来了。小慈以为她很快就能回到她 以往的生活里去了, 经历艰辛后会变得聪明些。但是不久, 她发现她被切开的部位开始感染, 又过了 几天, 小慈发起了高烧。她的姑姑想带她去医院, 可是她拒绝了, 她害怕医院里有人会发现她曾怀过 孕。第二天早上她的发烧症状更加严重了, 她的姑姑立刻叫了救护车, 但为时已晚, 小慈在到达医院 后几小时就死了。

安娜的故事: 安娜和她的丈夫住在玻利维亚偏远的安迪村里。她从来没有学过认字读书。她有一段时 间曾服用避孕药, 但是因为她丈夫的反对她就不再服用了。当安娜怀孕的时候, 她曾经去当地的健康 中心进行过一次孕期检查。但是到了中心她觉得很羞愧, 因为她不会西班牙语, 而当地的工作人员也 不会说盖秋亚族语。她觉得医生对她不关心, 只想让她离开。在她怀孕的第九个月, 安娜开始发现小 腹有疼痛感。一个做过助产士的邻居告诉安娜的家人说, 这个孩子的胎位可能不太正常, 并让他们带 安娜去健康中心。然而安娜一想到上次去健康中心做产前检查的情景她就不想去了, 况且她的丈夫听 
说健康中心要收一定的费用, 而他们的家庭却无力支付, 所以安娜一直呆在家里。十天后, 疼痛感越 来越强, 接生婆劝安娜的家人带她去健康中心。他们没法借到车, 所以他们只能用马拉的车带她去。 健康中心离小镇大约有15公里远, 对于马拉车来说, 算是一段很长的路, 在半路上, 安娜开始大出 血, 不久就死去了。

法蒂玛的故事: 法蒂玛想当一名教师。但是, 她在15岁时, 她的父母将她嫁了出去。她和她的丈夫, 艾里, 一个卡车司机, 住在一起。艾里的家人想要一个孩子, 对他们夫妇施压, 所以法蒂玛没有采取 避孕措施。在 5 年之内, 她生下了三个小孩。每次生孩子的时候, 有当地的接生婆为她接生。但是当 她怀第四个孩子到 8 个月的时候, 艾里正在外面开车送货, 此时法蒂玛疼得厉害, 因为家里艾里管钱, 所以法蒂玛没有钱乘车去健康中心。她焦急地等待他回来, 但当艾里回家的时候, 法蒂玛正因高烧而 浑身发抖, 身体极度虚弱, 艾里赶快安排将她送到医院, 可是当他们到达医院的时候, 法蒂玛已经死 了。

玛丽亚・克拉拉的故事: 玛丽亚 - 克拉拉和她的丈夫佩德罗还有她的侄子 (她哥哥的孩子, 她哥哥在 一次意外中去世）住在一起, 他们住在一个山村, 离山下最近的小镇挺远, 从家到医院有7小时车程。 当玛丽亚 - 克拉拉怀孕的时候, 她去做了检查, 医生告诉她, 应该在孩子出生两周前左右就到医院, 这样就不会再因为无法及时得到照顾而出现任何危险。当她快到预产期的时候, 玛丽亚 - 克拉拉对离 家有点紧张, 佩德罗抱怨妻子要离开那么久, 而他不会做饭。另外, 她知道大多数晚上佩德罗都会喝 得烂醉, 变得很疯狂, 所以她担心他会打她的侄子。她还担心佩德罗会跟他下班后常去的酒吧里工作 的那个女人鬼混, 因为一个邻居曾告诉她那个女人正在跟佩德罗约会。玛丽亚 - 克拉拉因而决定只提 前一周去医院, 在她出发前一天, 孩子出生了, 玛丽亚 - 克拉拉开始大出血, 而佩德罗未能及时将她 送到保健中心救治, 她就这样死去了。

亚茹内什的故事: 亚茹内什出生在埃塞尔比亚的一个偏远的社区。当她13岁的时候, 有一天她去拾柴 火时, 她被诱奸, 然后嫁给了一个叫做曾纳的男人。这个男人 32 岁, 是有两个孩子的鲤夫。之后不 
久, 亚茹内什怀孕了, 但她却没有任何人可以请教, 或可以说这个事。当她开始分婏时, 她吓坏了, 紧紧地抓着枕头, 她哭着呼喊妈妈, 眼泪不停地流, 后来她开始发高烧, 不停的流汗, 处于极度的痛 苦之中。一些邻居女人们来她家点亮了蜡烛, 开始为亚茹内什祈祷。曾纳请了村里的一个产婆, 但是 这个产婆从来没有接受过培训来处理这样的情况, 最后曾纳才请村上几个男人帮忙把亚茹内什送到了 医院。当她到医院的时候, 她的胎儿已经死了, 15岁的亚茹内什的生命也就此结束了。

胡珊的故事: 莎伊达, 一个来自巴基斯坦西北省曼丹市的女人, 给我们讲述了她唯一的女儿, 胡珊, 在13岁因为生孩子而死去的故事。“当胡珊 8 岁的时候, 她爸爸在一场交通意外中死了。那时我很担心 万一自己死了, 谁来照顾胡珊呢? 我想让她赶紧结婚, 过得幸福和安定。所以我给她找了一个对象: 隔壁村子里的一个农夫。胡珊对她自己婚礼很激动。三个月过后, 她就怀孕了, 我带她去见接生婆, 她给了一些止吐的药。我们可以分期付款。我们喜欢她的祈祷和所给的草药。胡珊干活很辛苦, 另外 她的饮食很差, 当时的迷信不允许产妇吃鱼和鸡蛋, 结果胡珊变得非常非常的瘦弱。我担心她怎么謷 得住分婏时候的剧痛。听到她开始大声呼喊疼痛的时候, 我们进去帮助她, 可是孩子依旧无法出来。 那个产婆变得很紧张, 拒绝再提供任何帮助, 告诉我们必须把胡珊送到医院, 在路上, 胡珊的身体渐 渐由发青变得冰冷。她死在了我怀中。我没有救活她和她的孩子, 我已经记不得我是怎么回到了家, 怎么举办了葬礼。那真是痛彻心扉。”

弗洛伦斯的故事: 弗洛伦斯, 24岁, 是一个靠种菜卖菜度日的单身女人。一天晚上, 在她从菜市场 回去的路上被强奸了。她很害怕, 没把这件事情告诉任何人, 那个月, 她没有来月经, 她意识到自己 因为那次强奸而怀孕了。尽管人工流产在她的国家里是违法的, 而且是在没有卫生保证的坏境下进行 的, 弗洛伦斯还是决定终止妊娠。她找到了一个人, 那个人在她的阴道里放了一个东西, 然后告诉 她会流一周血, 然后就会慢慢停止流血。但是, 一周过去了, 弗洛伦斯依然在流血, 而且她开始发高 烧。她去了医院, 那里的医生接待了她, 并给了她一些抗生素。起初, 弗洛伦斯因为怕被逮捕从而不 承认自己做了流产手术, 但最后她告诉了医生, 这个医生相信流产手术应该是安全和容易得到的。他 替弗洛伦斯难过，尽他最大的努力去挽救弗洛伦斯，但十天后弗洛伦斯还是死了。 


\section{活动46}

性传播疾病的事 实
性、性别、艾滋病和人权教育统一行动活动手册

时间：步骤1-3: 40分钟 步骤4-6: 40分钟

材料：海报纸、制作工具, 《指导手册》, 或者《指导手册》中的相关事实表: 性传播疾病、艾 滋病病毒和艾滋病、生殖道感染, 以及第七单元关于性病和艾滋病病毒的章节（更多《指 导手册》内容和关于性病艾滋病的其他资料可登陆：www.popcouncil.org/publications/ books/2010_ItsAllOne.asp )

准备工作：多准备几份以上提到的材料, 查找本地青少年可以在何处检测艾滋病及治疗的信息, 在黑 板上写下所有的讨论话题

概述：学生制作关于性病（包括艾滋病病毒和艾滋病）重要信息的宣传海报

目的：让学生描述性病是如何传播的, 如何阻止其传播, 去何处寻求帮助; 提高学生对性病检测 和治疗重要性的认识

\section{1. 解释本节课主要讨论性传播疾病 ( 包括艾滋病病毒和艾滋病)}

2. 将班级分成男女混合四个组，分配给每组一个话题 (或者让小组自行选择，尽量包括所有话题 ) 解释

小组需要协力制作一份相关选题的海报, 请发挥想象力;

海报内容越详尽越好。主要信息包括艾滋病检测、治疗和预防。学生可以参考其他资料和材料, 一些情况（比如关于 “迷” 这一话题的海报）, 可以通过采访同学来集思广益。 
3. 分发知识材料和海报所需用品，提醒学生用 10 分钟时间搜集信息，10分钟讨论并设计海报，另外 15 分钟时间制作海报。在大家调查、讨论和海报制作的时候，教师尽量在全场走动，并适当给予帮助。 如果觉得时间紧张，让他们再另找时间完成作业

下一环节;

4. 如果有必要，可以稍微延长制作海报的时间，完成他们的海报

5. 要求每个小组展示海报, 并回答提问

6. 将海报张占在教室内, 尽量多保留一段时间

\section{海报话题}

- 性病之迷;

艾滋病病毒和艾滋病之迷;

- 学会自我保护;

关于艾滋病病毒和艾滋病的五个重要信息;

- 什么是衣原体;

- 性病与性别;

- 衣原体和淋病如何影响未来生育;

乳头瘤与疱疹: 两种主要的性病;

- 什么是生殖道感染。 
性煡唇灵 


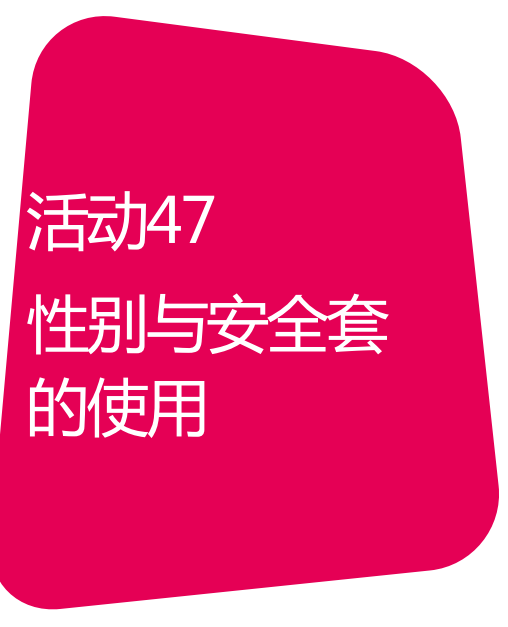

时间：步骤1-4（为什么有些人不用安全套）：40分钟 步骤5-7 (安全套的正确使用)： 15分钟

材料 黑板、粉笔; 教师参考答案; 步骤1-4: 工作表复印件; 步骤5-7: 配对卡。如果合适, 准备男用和女用安全套用以演示

准备工作 : 确认在当地是否可以获得女用安全套, 从哪获取? 复习一下在《指导手册》最后关于避孕 的事实列表中男用和女用安全套的信息，或者在其他可靠书籍中寻找。步骤 1-4：阅读工 作表和教师参考答案要点。步骤5-7: 准备配对卡（见“安全套的正确使用步骤”）

概述：学生分析使用安全套的障碍 (包括性别) 和克服这些障碍的方法, 学习如何使用安全套。 这个活动适用于那些已经基本了解安全套是预防性病、艾滋病病和意外怀孕的有效方法的 学生

目的 : 使学生能够描述安全套使用的障碍及怎么应对这些障碍; 描述安全套的正确使用方法; 增 强批判思维技巧

\section{步骤}

\section{1. 解释 (写下主要的问题 ) :}

- 今天我们将讨论安全套的使用。我们对安全套都知道些什么; ( 探讨：安全套能预防性传播感 染、艾滋病病毒和意外怀孕; 它是男性使用的 )

- 谁会使用安全套? 有没有女用安全套; ( 探讨：事实上, 也有女用安全套, 提供相关信息。)

- 为什么了解安全套很重要; ( 探讨: 每天都有人死于艾滋病和不安全流产并发症, 如果使用了安 全套这些死亡本来是可以避免的。安全套能够避免怀孕、艾滋病和其他感染）

- 如果人们知道安全套, 是否意味着人们会使用? 


\section{2. 将全班学生成对分组并分发工作表}

解释 :

- 人们不使用安全套或不正确使用安全套的原因有很多。工作表中描述了9对伴侣的真实经历, 同时 也提供了对这些伴侣可能有帮助的建议;

- 和你的搭档一起读一遍每对伴侣的情形, 然后决定哪种或哪几种解决方式适合他们的情形。有什么 方法能帮助他们下一次正确使用安全套?

3. 把全班集合并讨论工作表中描述的每对伴侣的情况。如果时间允许对伴侣C、F和I（涉及两性不 平等问题 ) 可进行更深入讨论

4. 对9对伴侣的情景进行评论后，围绕以下问题进行进一步讨论：

- 谁应该为使用安全套负责;

- 为什么女孩或成年女性需要获取女用安全套; ( 探讨: 如果她们的伴侣不愿使用男用安全套采取的 自我保护措施。)

- 人们如何保证一旦需要就会使用安全套。

5. 把班级分为两到三个小组 (每组约12到16人)

介绍主题:

- 人们不使用安全套的原因之一是他们不知道如何使用。正是这个原因会导致严重的健康问题，所以 我们现在要学习安全套的正确使用方法。到了学习使用安全套的年龄并不意味着你就要有性行为, 但你最好在需要用到之前学会如何使用安全套, 而非你在有了性行为之后, 那样就太晚了。使用安 全套时, 你可以在事前采取一些步骤, 也就是在处于亲密气氛之前。有些步骤可以在你已经处于亲 密气氛时采取, 但是一定要在性行为即将发生之前。你应该知道在性行为中如何使用安全套, 知道 
使用完后如何处理安全套。（在黑板上写下 “之前”、“即将开始性行为时”、“性行为中”以 及 “性行为后” )

6. 解释这个活动

- 我们已将正确使用男用安全套的所有步骤分解, 并将每个步骤写在一张卡片上。我将会为每一组 发一套配对卡, (一定要在分发卡片之前将卡片次序打乱。) 每个小组都必须一起将这些步骤整 理成正确的次序。

- 如果可能, 小组的每个人都拿着一张不同的配对卡, 然后将每组分为四小组 (指向黑板上的四个 选项）。第一分组应该包括手里拿着第一步骤卡的同学一一是指那些性行为事前必须做的。第二 分组的同学的卡包括了性行为前需要立刻做的步骤。第三分组同学的卡要包括性行为中的步骤。 第四分组同学的卡显示了性行为后的步骤。每个分组要将步骤按顺序排好, 并根据手里拿的卡片 站成一排; ( 注: 第一分组可能会有点困难 )

- 你们有五分钟时间。开始!

7. 五分钟后，喊停，将所有小组集合起来。请同学们解释步骤的顺序。当顺序不对时，打断他们并提问 ( 参见回答要点)。回答同学们提出的任何问题

\section{学生工作表}

帮助人们使用安全套的方法

下面是 9 对没有正确使用安全套的伴侣。针对每一对，想一想怎么样可以帮助这个人或这对人。从下列 选项中选择一个或多个解决办法, 并在序号上画圈。例如, 画圈以表示 “男孩和女孩更多平等和分享 权力”。( 你可以多圈几个数字, 如果你认为这对伴侣需要更多的解决办法 ) 
解决办法1 正确使用安全套的信息

解决办法2 男女平等、分享权力

解决办法3 更好的交流技巧

解决办法 4 如何获取安全套的信息

解决办法 5 关于艾滋病病毒和怀孕更实际的考虑

解决办法 6 一个讨论性行为更安全的地方

A对伴侣: 这对伴侣有性交, 也使用安全套。男孩射精后, 他静躺了五分钟。他的阴茎开始松软变小, 他稍一动, 却吃惊地发现自己的部分精液从安全套里流入了女友的阴道口。

A对伴侣需要: 123456

B对伴侣: 这对年轻人决定发生性行为并准备使用安全套。其中一个去当地超市购买安全套, 但没有 发现男用或女用安全套。另一位想学校可能会有安全套, 但护士说没有。他们都希望对方会找到安 全套。周末, 他们开始做爱。他们都觉得把发生的事情告诉对方太尴地, 所以就避而不谈安全套的事 情, 并且在未用安全套的情况下发生了性行为。

B对伴侣需要: 123456

C对伴侣: 这两个年轻人想做爱。女孩告诉自己的男友, 她买安全套了。但是男方孩说: “没门! 那样 感觉很糟糕! ”她想坚持, 但是他开始生气了, 说她像个小孩, 并问她是不是真的爱他。最后, 女孩 
屈服了, 他们没使用安全套就做爱了。

C对伴侣需要: 123456

D对伴侣: 这两个年轻人想发生性行为并且同意使用安全套。但是到了该使用安全套的时刻, 男孩摸索 着安全套的包装袋, 勃起失败了。这次, 他们没有性交。当他们再次在一起的时候, 他开始紧张, 生 怕勃起失败。他告诉女朋友, 安全套破坏了气氛, 于是他们决定不用安全套做爱了。

D对伴侣需要: 123456

E对伴侣: 这两个年轻人决定发生性行为。他们都觉得使用安全套是个好主意, 但是担心如果自己提议 使用安全套的话, 对方会认为自己是 “脏” 的。最后, 谁也不知怎么说出口, 他们就在没有使用安全套 的情况下发生了性行为。

E对伴侣需要: 123456

$F$ 对伴侣: 这个男孩16岁, 是某帮派成员。他想离开帮派, 但是这样做非常困难而且很危险。不过如 果他当爸爸了, 帮派就同意他离开。男孩向他15岁的女朋友解释, 如果他们有了孩子, 会拯救他的生 命, 而且自己会是一个好爸爸。女孩不知所措, 她想完成学业, 而且觉得自己还没有当妈妈的准备。 但是她同时又觉得应该帮助自己的男朋友，于是他们在没有使用安全套的情况下发生了性行为。

F对伴侣需要: 123456 
G对伴侣: 这两个年轻人决定发生性行为。男孩问女孩是不是要采取避孕措施, 但是女孩说自己的月经 刚结束, 不会怀孕的。

G对伴侣需要: 123456

$H$ 对伴侣: 这两个年轻人决定发生性行为。他们讨论是否应该要使用安全套来预防感染艾滋病病毒, 但 是他们都认为如果得了艾滋病, 他们会知道的。于是他们在没有事用安全套的情况下发生了性行为。

H对伴侣需要: 123456

I对伴侣: 一个17岁的女孩与 25 岁的男人有性关系, 这个男人送她送礼物, 有时候给她钱花。他有时会 使用安全套, 但这次他身边没有。她想他们应该在下次有安全套的时候再做, 但是他发誓没有套没关 系。她这个星期已经从他那里拿了钱, 所以觉得不能拒绝。于是他们在没有使用安全套的情况下发生 了性行为。

|对伴侣需要: 123456

教师参考答案

教师应注意: 以下是每对伴侣问题的主要解决方式。每一个案例, 学生可能还有其他的解决方法。教 师应灵活处理, 但最少应保证以下的答案包含在学生的最终答案里。 


\section{教师参阅信息 \\ 正确使用安全套步骤 \\ 注意: 准备 16 个卡片, 在 每个卡片上写下一条内容, 不 要在卡片上标记任何序号, 确 \\ D对伴侣 方法6 \\ F对伴侣方法2 \\ G对伴侣 方法5 \\ H对伴侣方法5 \\ |对伴侣方法2} 保发放卡片时，步骤1-5是随 机的。
B对伴侣 方法4、方法3

C对伴侣方法2、方法3

E对伴侣 方法3、方法5

准备工作

1. 与伴侣讨论什么是安全性行为

2. 购买或者在社区、医疗机构免费领取安全套 (及润滑剂)

3. 把安全套存放在干燥阴凉的地方 (不要放在钱夹中)

4. 检查安全套的保质期, 确保安全套没有过期

5. 练习带上安全套, 这样真正使用时才会觉得舒适

即将发生性行为之前

6. 全身心投入前戏中, 包括触摸阴蒂, 能够帮助阴道润滑 
7. 小心慢慢撕开安全套包装, 千万不要划伤安全套 (千万别用牙咬开 )

8. 当阴茎勃起, 挤出安全套前部储精囊中的空气, 从龟头向阴茎根部带上安全套

9. 从龟头开始向下拨安全套, 使其套住整个阴茎

10. 如果阴道仍然很干涩, 再加入一些前戏，还可在安全套上涂抹水溶性润滑剂或使用唾液湿润。千万 不要使用凡士林或者其他油性润滑剂, 因为有可能造成安全套腐蚀或破裂

\section{在发生性行为中}

11. 如果安全套破裂, 男性应该马上抽出阴茎 (若男方已射精, 教师可以提醒学生一些性行为后避孕的 补救措施 )

12. 在射精以后, 阴茎仍然处于勃起状态

13. 握住安全套根部

\section{性行为结束后}

14. 捏住安全套根部, 轻柔地取出阴茎

15. 小心地取下安全套, 捏住安全套的储精囊, 避免精液流出

16. 在安全套根部打结或用卫生纸包住, 并妥善处理 


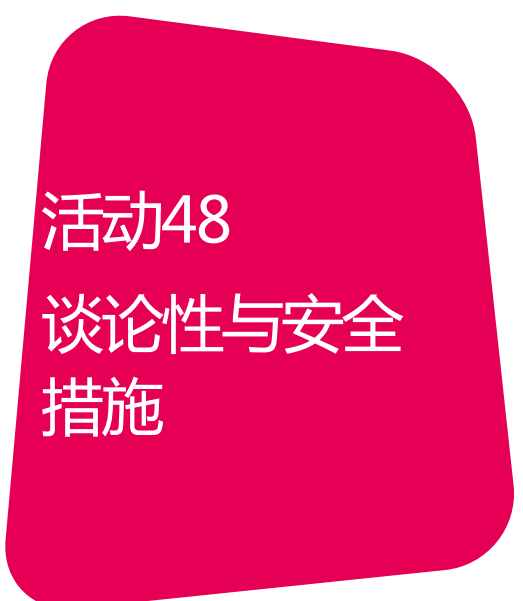

时间：步骤1-3: 40分钟 步骤4-10: 40分钟

材料 黑板、粉笔

准备工作：检视所设定的场景并根据实际情况作相应调整（如伴侣已订婚），合理安排时间

概述：学生练习如何开始就性安全和性健康一一这一常被忽视却是很重要的话题一一展开对话 ( 注: 这个活动与活动38有部分重叠, 教师选其一, 也可两个都开展 )

目的: 使学生学会就有关性和性安全问题的话题进行思考, 并练习如何开始、展开、拓展关于这 些话题的对话。增强学生批判性思维的能力

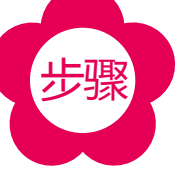

1. 通过以下的问题引入活动 :

- 今天我们将思考并练习如何引出一个有关性安全问题的对话。谈论与性有关的话题容易不容易? 为什么;

- 如何让这样的讨论更简单?

2. 让学生组成小组 ( 最好是一男一女结对成组 )。在黑板上写下以下话题 :

- 要不要发生性行为; 之前的吸毒经历;

- 我之前的性经历;

- 性病、艾滋病; 
3. 解释:

- 在两人小组中, 你需要练习如何发起一个难以启齿又至关重要的话题。针对黑板上的每一个话题, 讨论如何向潜在的性伴侣引出并讨论这些话题, 写出至少一种打开话匣子的方式;

- 同时讨论决定一对伴侣第一次性行为的对话应该在什么时候开始? 当你们见面时还是初吻后? 还是 当你们已经开始性行为时? 记住, 人们不需要一见面就议论一切。

4. 针对每一个话题请一个组分享他们讨论的情况，记录在黑板上。并问其他同学：

有没有人有不同的建议; (把不同的建议加到黑板上的列表中 )

- 你认为什么样的主意会是有效的? 为什么? 你认为这些建议哪些是不合适的? 为什么?

- 这样的初次对话应该在两人交往的什么时候开展? 为什么?

5. 在回顾了五个话题以后, 问 :

- 如何使涉及这些话题的交谈变得更容易轻松;

- 现在我们要练习思考现实生活中这样的对话如何进行。

6. 解释:

- 第一位同学发起对话, 但第二位同学需要让这个对话更加有难度, 他可以表现得很尴尤或不同意, 或者试图躲避这样的话题;

- 第一位同学的任务就是努力将对话继续, 哪怕继续一点点。

7. 邀请两位志愿者表演他们就第一个话题的对话 ( 是否要发生性行为)。向同学说明

- 为你扮演的角色取个名字, 尽量表演得真实;

- 以下是你们的剧情 (可以用你们起好的名字代替里面的名字 ): “阿里和丽亚已经约会一段时间 
了, 而且有了亲密的身体接触, 但还没发生过性行为。他们都不清楚对方之前的性行为和是否吸 过毒。阿里认为两人应该更多地享受性带来的快乐, 但他总是担心会感染艾滋病病毒。现在他们 正在散步。”

- 其他每个同学都要记录这两个志愿者是如何交谈的。

8. 针对以下问题，开展一个小型讨论

- 在他们的对话中, 哪些部分表演得较好? 哪些也许可以是另外一个样子的;

- 这个对话真实吗;

- 你对阿里和丽亚有什么建议吗?

9. 如果时间允许，就下述不同剧情，重复步骤6-8

- 阿加里询问莫是否可以发生性行为, 他们可能态度一致, 也可能没有达成统一的想法;

- 卡罗发起对话, 和玛议论双方过去是否有吸毒或者性经历;

- 亨利和米亚已经讨论过性的话题, 并且想发生性行为。亨利发起对话, 和米亚讨论使用安全套的 问题（之前私下告知表演者亨利在剧中不想在没有用安全套的情况下发生性行为，再私下告知另 一位表演者, 剧中米亚认为没有必要使用安全套 )。

10. 通过以下问题，总结本次活动。将回答记录在黑板上

- 在你发起诸如这样的对话之前, 你需要自己先考虑什么内容; ( 探讨: 你有什么感觉, 你想要的是什 么，你想说些什么）

- 成功的对话有哪些技巧;

- 有礼貌地拒绝的技巧有哪些;

- 双方各有什么权利; ( 探讨: 表达观点的权利, 拒绝的权利, 保护自我健康的权利 ）

- 恋人之间, 谁应该承担发起话题的责任? 为什么? 
时间：步骤1：10分钟（前一天）步骤2-4：40分钟（可灵活掌握）

材料：黑板、粉笔。故事材料 (如果没有演讲者)

准备工作: 邀请艾滋病病毒感染者 (理想情况是一男一女)。如果不行, 邀请一个在艾滋病病毒和艾 滋病防治领域的工作人员, 或者关注艾滋病病毒感染者的人。邀请他们讲述自己的亲身经 历。告诉演讲者学生们的年龄、他们对艾滋病的了解情况及预计演讲/提问的时间。建议 演讲者讨论以下话题: “了解自己的艾滋病状况”、“公开自己的艾滋病病毒状况”、 “差辱/歧视”、“如何获得理解和支持”、“保健和治疗”、“日常生活”、“艾滋病 病毒感染的男人和妇女有哪些方面的不同” 等

( 在演讲者到来之前先开展步骤1活动)

概述：学生聆听一位艾滋病病毒感染者的真实故事

目的 : 使学生了解艾滋病病毒感染者和艾滋病患者, 并且同情关心他们

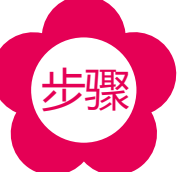

演讲者到来之前

1. 告知学生在接下来的一节课中，有一位或几位特邀演讲人要和大家分享自己的艾滋病经历。提问 : “你希望听到什么内容?" “你有什么样的担心和恐惧?"，有必要的话，针对每种担心，向学生解 释相应的艾滋病常识。提醒学生遵守最基本的原则。如果还未制定原则，请参与者头脑风暴，得出一 些应该如何正确对待演讲者的规则

备选活动: 如果不可能在社区邀请到一个艾滋病患者, 你可以使用资料中 “真实的故事”。让学生为

\section{活动49}

艾滋病只是一个

名称 
- 每个角色取一个当地的名字, 并且大声朗读这个故事。允许他们提问, 然后继续第3、4步。 演讲过程中

2. 简单介绍演讲者，并感射他的到来，告诉大家演讲大概会持续多久

3. 预留时间给学生提问。然后再次感谢演讲者并送别 演讲者离开以后

4. 针对以下问题组织讨论：

- 在演讲过程中你的感受和印象是什么;

- 演讲中的哪一部分对你来说最有意义, 哪一部分最令你吃惊;

- 从刚才的演讲中, 你对感染了艾滋病病毒后会是怎么样有了哪些新的认识;

- 演讲者刚才讲到的是一种怎样的感受;

- 演讲者都遭遇了哪些困惑和固有、刻板的看法;

- 你还有其他问题吗;

- 你今天学到和经历的重要的部分是什么? 你今天学到的东西将会从哪些方面改变你? (探讨: 在 以后的生活中, 我将改变自己对艾滋病病毒感染者和艾滋病患者的态度, 我今后也会更加小心地 防止感染艾滋病病毒。1 
米文芝的故事: 我是一个来自赞比亚的妇女, 今年22岁。感染上了艾滋病病毒, 这种病毒会导致艾滋 病。在我很还小的时候, 我的父母就因为艾滋病去世了, 我从小由姐姐抚养长大。19岁时, 我怀孕了 并在当地医院接受产前检查, 他们让我进行艾滋病病毒检测。当我被告知自己的检测报告结果呈阳性 时, 我简直不敢相信这个事实。我觉得自己身体健康, 看起来也非常健康, 我感觉那不是真的, 我可 不想死。当我告诉我男朋友的时候, 他一笑了之, 说: “别瞎说了, 你哪里看上去有病? 他们只是吓 唬你而已。” 接着他也去做了检测, 结果显示他也感染了艾滋病病毒。幸运的是, 我姐姐是个有爱心 的人, 她帮助我支付了医疗费用并且在其他方面也一直支持和帮助我。我下决心不让艾滋病病毒成为 我人生的羁绊, 我要与之搏斗。幸亏有了这次检查, 我才开始进行抗逆转录病毒治疗, 这种治疗使我 保持健康, 使我的小宝宝在出生时没有感染艾滋病病毒。最残酷的事是周围人因为我的病而对我不友 善。我不知道自己还能像这样保持健康多久, 但我现在生活得很充实, 我是一个好母亲, 我有一份工 作要做, 那就是教导青年人保护自己和他们的伴侣避免感染艾滋病。

布瑞特的故事: 18岁时的我聪明伶俐, 还留着朋克式的发型。白天我在大学上课, 晚上则在酒吧和 
地铁里度过。仅仅两年后, 当我站在珠宝店旋转门之间, 通过投币电话得知自己感染了艾滋病病毒 时, 我震惊了。但是, 我健康上缺乏的东西, 我从青春中得到了补偿。我勇气十足, 很理想主义。我 相信我们一一患者、医生、科学家和研究人员, 只要真正想做的话, 就一定能够治愈艾滋病。虽然这 听起来很不现实, 但当时我们需要鼓励。理想主义让我在希望渺茫的时候坚持下来。我一直想写一些 东西, 确诊以后, 我确切地知道我要写什么, 然后全身心投入到写作中去。我筹钱办了一本名为《新 生》的杂志，我的想法是办成全球第一份专门关注艾滋病病毒感染者生活方式的杂志。有时候我感到 精疲力竭, 但是写作和朋友是我的动力。我的整个成人生活都是在艾滋病病毒阳性的状况下度过的。 当然，我们还没有找到治愈艾滋病的方法。但2008年人们研制出了遏制艾滋病病毒蔓延的一种极好的 药物, 而且这种药物正逐年得到改进。但这种药物也并非完美。它让身体很难受, 但也不是说从此你 可以放纵自己, 破罐子破摔。为了健康, 记住: 开车系好安全带、不抽烟、少喝酒, 在需要的时候接 受心理治疗、锻炼身体，切记务必使用安全套，并要诚实地对待自己生活各方面。 
时间：步骤1-4: 45分钟 步骤5-6: 45分钟

材料 黑板、粉笔

准备工作：在第一步做深入探讨时, 想一些开放性 (不光答是与否) 的问题。如果可能的话, 找一些 学生所在地的有关艾滋病传播的数据和信息。这个活动可能会在某些学生中引起较强反 响, 思考如何给他们以适当帮助。尊重每个人的隐私, 不要强求他们说自己的故事

概述：学生讨论在人们告知性伴侣自己的艾滋病感染状况上应承担多少责任。让学生们权衡个人 隐私权和保护自己不被伤害的权利这两者之间复杂的利害关系。通过创作，他们探求自己 对于这两种两难境地的感受 (注意: 本活动之前, 学生应当掌握基本的人权、知情同意、 艾滋病病毒和艾滋病的常识 )

目的：让学生批判性地思考在对他们的性伴侣透露自己的艾滋病状况问题上应承担多少责任 或义务

\section{步骤}

1. 通过以下问题介绍本次活动 ( 在黑板上记录学生的回答)

- 今天我们将讨论一个敏感和复杂的问题: 人们是否有义务向自己的伴侣透露自己是艾滋病病毒 感染者;

- 你认为那些知道自己感染艾滋病病毒的人是否总会把这一情况告诉自己的性伴侣;

- 人们不告诉对方自己感染艾滋病病毒是出于什么原因呢; ( 探讨: 保护个人隐私, 害怕被羞辱, 抛 弃和歧视等 ) 
- 你认为人们不去做艾滋病病毒检测的主要原因是什么; ( 探讨: 他们害怕被证实自己已经感染艾 滋病病毒, 认为去诊所很尴炌; 并不知道哪儿有免费检测点; 因为感觉和看起来很健康, 所以不 相信自己感染艾滋病病毒; 去做检测的时间和上班上学的时间有冲突，不相信检测结果会被保 密)

- 为什么人们应该去做艾滋病病毒检测 ; ( 探讨: 如果结果呈阴性, 他们可以松口气; 如果检查结 果呈阳性, 他们可以及时接受治疗, 使伴侣今后不致感染; 告知以前的性伴侣, 让他们可以及时 去检测 )

2. 请学生拿出笔记本，向大家解释 :

- 我们已经考虑过一些导致人们选择不接受检测, 或选择不告诉伴侣自己的艾滋病病毒感染状况的 原因。你们的任务是写一个更深入讨论该主题的短小故事，不要超过3页;

- 你们的故事应该写些什么? 你们的故事可以是关于一个选择不去接受检测的人, 或写一个被其伴 侣感染上艾滋病病毒的人, 因为其伴侣没有透露他或她其实是一位感染者, 或者也可以是关于一 个艾滋病病毒感染者主动将自身的感染状况告知性伴侣;

- 你们写的故事应该探索哪些议题和感觉? 故事应该有情节起伏, 能够展现你的主角是怎样做出决 定的。他或她有什么感觉? 发生了什么?（这个决定怎样影响主角的生活或其他人的生活）思考 这个主角的感受 (害怕, 得到支持的, 孤立无援的, 轻松的, 后悔的, 被爱的, 被感谢的, 苦涩 的)

- 你们写的故事是真实的还是虚构的? 故事可以完全是你创造的, 或者是基于你自己或你认识的人 情况写的, 但不允许使用任何人的真实姓名。最后再强调一下: 这些故事不要太长, 但必须是严 肃的。这是个需要深思熟虑的任务, 而不是开玩笑的。如果课堂时间内没有写完这个故事, 可在 课后完成。 
3. 让学生开始思考并写故事。如果你准备让学生朗读他们的故事，在他们开始之前就告诉他们。告诉 学生如果他们在构思故事时有什么困难可以跟教师说（例如你可以建议 :一个男孩很害怕去做检测， 因为他的双亲死于艾滋病;一个女孩不敢告诉自己的男友自己因为被强奸而感染了艾滋病病毒，因为 她的男友相信在他们相识之前她还是处女。)

4. 当场收集学生们的故事，或者让他们将故事写作作为课外作业去完成。但下节课前 (下一节将要求 学生大声朗读自己的故事 ），你先亲自把所有故事都读一遍，以确保每个故事都是合适的和匿名的

5. 邀请学生朗读自己的故事 ( 并不是每个人都必须朗读 )

6. 讨论以下问题以结束此次活动 :

- 在听了某个同学的故事或根据教师建议写的故事以后, 你们有谁开始从不一样的角度看待这个 “进 退两难” 的情况呢; ( 探讨: 你们现在把接受艾滋病病毒检测看得比以前更困难还是更容易呢? 和 之前相比, 你现在认为 “让性伴侣知情” 更重要还是更不重要? 现在你觉得如果你的性伴侣向你坦 白了他感染艾滋病病毒的事实, 你的态度与以前相比是否会有所不同? )

- 透露一个人的艾滋病病毒感染状况是不是件明明白白的事?

我们怎样来保证我们尽可能做到对伴侣和我们自己诚实, 以确保自己和他人的健康?

- 每当人们去诊所时, 诊所是否应该提供或者大力推荐自愿的、匿名的艾滋病病毒检测呢? 或者应该 只是有人明确要求做检测时, 医务人员才给他们做艾滋病病毒检测;

- 还有谁要做最后评论? (试图为这个活动作总结性评论: 艾滋病在全球范围内流行, 这需要我们每 个人诚实、有同情心、自律、勇敢和乐观。) 


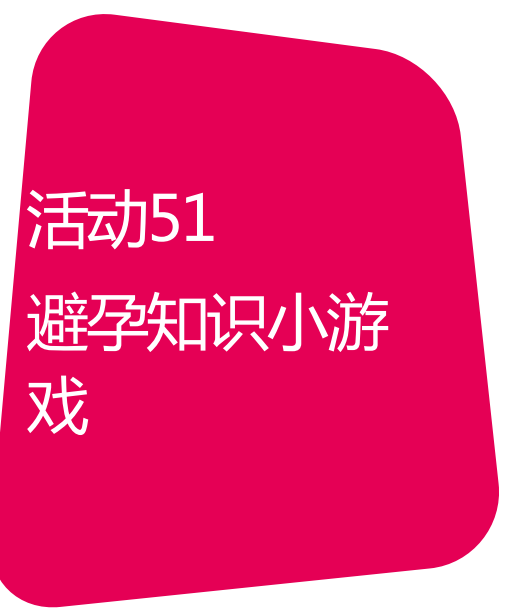

时间：45分钟

材料 黑白、粉笔、字谜题目、写有避孕方法的纸条、一个口袋、八份选自《活动指导手册》 （本卷配套书）或其他可靠教材的关于避孕方法的资料、一只计时表、一份小礼品（选择 项 )

准备工作 : 复印后面的字谜 (包括答案) 。阅读避孕方法的列表, 删去练习时不适用的几种。在课 前, 先带领学生阅读列表中的一些避孕方法。将每种避孕方法名称写在一张纸上, 折起来 放入口袋中。阅读第三步的注释并做好相应的准备。教师自己也要事先复习避孕的知识

概述：学生通过字谜和小游戏复习有关避孕方法的知识。并讨论男女双方在避孕方面的责任 (注 意: 这个活动可以作为对避孕方法的介绍或回顾 )

目的: 复习、强化避孕知识, 增强学生写作能力

\section{步骤}

课外作业（在本次活动之前布置）

完成后面的“避孕方法” 字谜（给每个学生发一份空白字谜）。

接下来一天, 开始第一步活动:

1. 以小组为单位回顾字谜答案，教师随时准备回答学生提出的问题

告诉学生接下来大家将玩一个 “五个问题” 的小游戏。这个游戏会考察学生关于不同避孕方法的知 识。分成三个小组，解释 :

- 这个口袋里有一些纸条, 每个纸条上写有不同的避孕方法。第一小组先从口袋里抽取一张纸条。 
- 第二小组和第三小组将猜测第一小组手里拿的纸条上写的是哪种避孕方法。要找出答案, 你只能问 “是” 还是 “否” 这样的问题。（确保每个人都明白 “是”与 “否” 是指的什么）, 先由第二组提 问。

2. 解释游戏规则 (注意: 如果学生对避李方法有所了解，他们可以采取 “是” 或 “否” 来帮助猜出正 确的答案【避李方法】。如果他们连最基本的避孕方法都还不了解，每一个小组则需要一份资料作为 指导）

- 为了确保做出正确回答, 回答问题的小组可以参考关于避孕方法的资料, 但只有30秒的时间来回 答 “是” 或 “否”。在给出回答之前, 猜的小组可以组内小声讨论。抽取的纸条必须让教师看到, 这样, 如果小组给出错误的信息时, 将被扣除 0.5 分。切记: 你只能回答 “是” 和 “否”。最后让 你小组的某个成员记录你们回答了多少个问题, 并且必须在五个问题以内猜出这个避孕方法, 否 则, 此轮游戏结束;

- 在第一组回答了第二组提出的第一个问题后, 第二组可以试着猜测说出避孕方法的名称, 第二组猜 错了 (或者放弃猜测), 第三组可继续提问。第二组和第三组就五个问题轮流提问, 直到猜中正确 答案为止 (猜中组加一分);

- 如果每个组在问完五个问题后还未猜出正确答案, 第一组告诉大家正确答案是什么, 并指出该避孕 方法的要点在哪里, 并得一分。

3. 解释如何继续游戏

- 第一组回答完问题以后, 轮到第二组抽纸条, 此时由第一组和第三组提问。以此类推直到时间截止 或者所有的纸条都被猜完。

大家都明白游戏规则了吗? (如果必要, 可用一张写有某种避孕方法的纸条做个示范)

4. 指定一个计时员，每次提问和回答的时间是30秒。再指定一名记分员，在黑板上为每小组记分。 
5. 让第一组抽取一张纸条并且给该组一份避咨资料；确保纸条不被重复抽取；教师自己保留一份资 料，以便及时更正学生的错误。

6. 在猜中一种避李方法后，或者五个问题都被回答后，暂停游戏。给赢的小组记分，然后请第二组从

课外作业 :

写一段话来回答下面的问 题: 每个人都需要了解避孕 知识吗? 防止意外怀孕是谁 的责任? 你可以直接阐述你 的观点，也可以通过故事间接 阐述。

\section{课堂练习资料}

\section{字谜题}

规则 :

根据所给提示填写英文 单词。横排与竖排交错处 的格子所填字母应该共同 适用于两个单词中。

横排包括1, $4,6,7,9,11,13,14,16$

竖排包括 $2,3,5,7,8,10,12,15$ 口袋中抽纸条。让各小组轮流这么猜答约二十分钟，或者玩到本节活动课结束前十分钟。

7. 公布最终获胜的小组并颁发礼品 ( 如果准备了的话 )。

8. 给整场活动留出5-10分钟讨论时间。

- 今天你学到了哪些以前不知道的、但现在你觉得有趣的避孕方法;

- ( 以下可留为课外作业) 每个人都需要了解避孕知识吗? 谁应该为意外怀孕负责?

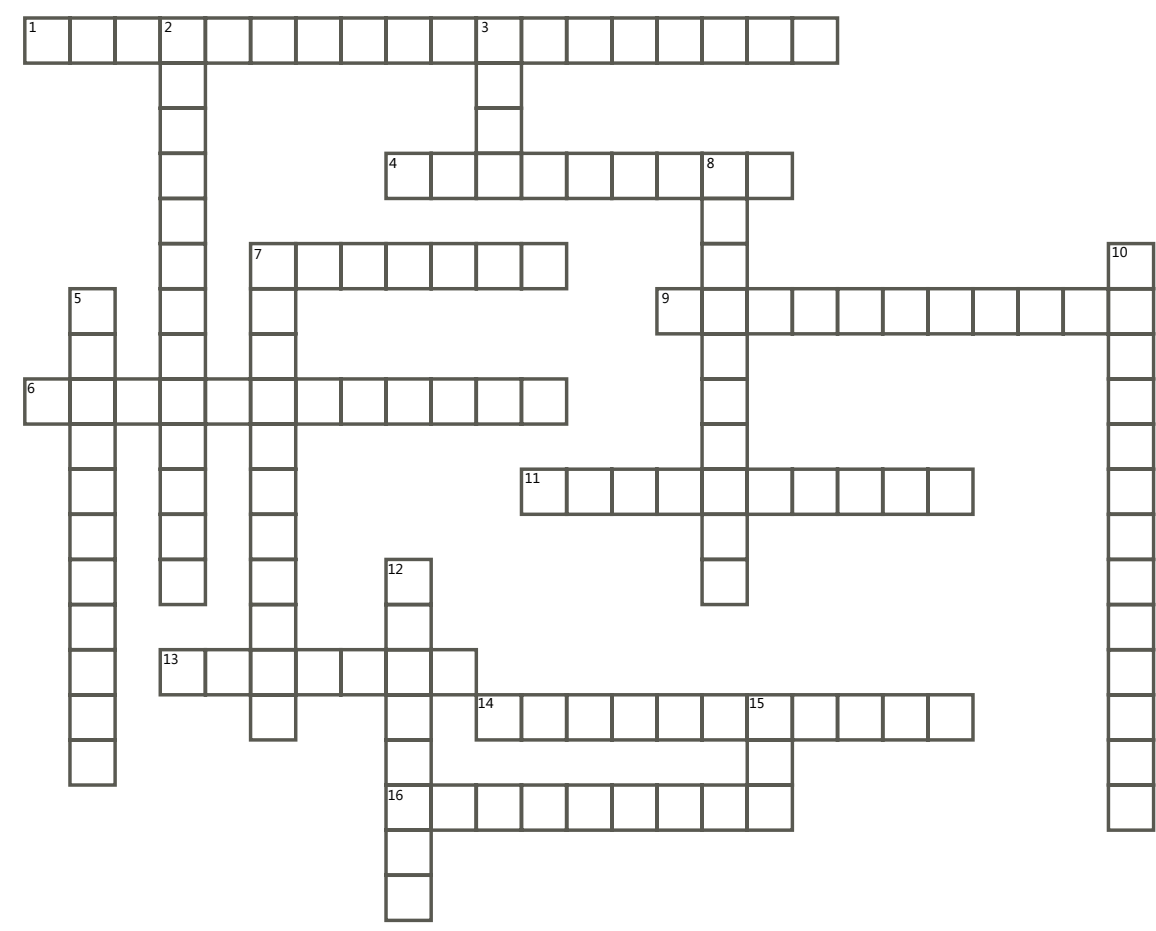


横向

1. 女人或者女孩将其贴于身上, 但其对预防性病或艾滋病并无功效（两个字）

4. 一种防止男子释放精子的外科手术

6. 女子放入阴道防止精子进入的一种鞘状套

7.一个插入女人或女孩手臂的小棒

9. 女人或者女孩用一个温度计来测量其是否怀孕的方法

11. 在射精前将阴茎抽出

13. 女人或女孩每天服用以防止怀孕

14. 一种女人或女孩放在阴道内的环状物, 但其不能防止性病或艾滋病（两个字）

16. 一个放在阴道覆盖整个宫颈的橡皮杯,其中有杀精剂

竖列

2. 一种女子的输软管切除手术以防止受孕 (两个字)

3. 插入子宫, 并且呈 “T” 字形 (缩写, 复数形式)

5. 各种放在子宫中防止受孕的物质 (复数)

7. 阶段性给女人或女孩注射来防止排卵或宫颈粘液变稠（复数）

8. 男人或男子在做爱时套在阴茎上, 它能够预防怀孕、性病和艾滋病（两个字）

10. 女人或女子能够通过这个物质的数量和稠度辨明是否怀孕

12. 女人或女孩可以通过将其生理周期记录在什么地方来预测是否怀孕 
224 青春健康教育指南 性、性别、艾滋病和人权教育统一行动活动手册 9

15. 从自然的方法来产生母乳喂养 ( 缩写 )

答案:

横向

竖向

1. 避孕贴片

2. 输卵管结扎

4. 输精管切除

3. 内节育器

6. 女用避孕套

5. 杀精剂

7. 移植

7. 注射剂

9. 体温

8. 男用避孕套

11. 抽拔

10. 宫颈黏液

13. 药片

12. 日历

14. 阴道环

15. 哺乳闭经期避孕法

16. 隔膜

（教师参考资料 世界各地的避孕方法 ) 
临时性、由使用者控制的方法 ( 主要是阻止精子与卵子结合)

- 男用安全套;

- 女用安全套;

- 阴道隔膜;

- 子宫帽;

- 杀精剂。

\section{在人体内起作用的方法}

1. 短效、由使用者控制的：

- 口服避孕药 (片);

- 紧急避孕药。

2. 长效的 :

- 长效避孕注射针;

- 荷尔蒙阴道环;

- 荷尔蒙贴片;

- 荷尔蒙皮下埋入剂;

- 宫内避孕环。 
自然方法 (要求采取特定的行为且了解自己的身体情况 )：

- 哺乳闭经期避孕法;

- 体外射精避孕法;

- 宫颈粘液法 (以此判断安全期 ) ;

- 体温测量法;

- 安全期避孕法 (通过日历规律的生理周期, 由此判断安全期);

- 节欲;

- 手淫。

永久性的手术法:

- 输卵管结扎 (或者其他女性节育手术)；

- 输精管结扎。 
时间：60分钟（也可分成两节课）

材料 黑板、粉笔。从 “流产案例” 中引用一个材料, 分发给各组 (或者复印所有案例, 分 配给每组一个案例 ), 或者《指导手册》 ( 登陆网站www.popcouncil.org/publications/ books/2010_1stAllone.asp ) 第七单元关于意外怀孕和流产部分 )

准备工作：阅读上述材料中妇女选择流产的原因, 必要时适当调整内容使其适应本地文化。确保案例 反映了选择流产的诸多原因。熟悉一下本地有关流产（特别是青年人流产）以及儿童抚养 的法律

概述：学生阅读案例并且讨论妇女流产的原因

目的 : 请学生说出至少三种妇女和女孩选择流产的原因。增强批判性思维能力和对话技巧

\section{活动52}

站在她的立场：

停止娃娠的决定

\section{步骤}

1. 通过以下问题引入活动

- 今天我们要讨论一些人生中重大而复杂的决定。回想一下你或者你亲近的人必须做出某个困难的、 而且他人可能不同意的决定时的情景; ( 给学生一些时间思考)

- 你有什么感想? 有人 ( 或者你想到哪个人) 支持你吗?

- 如果没有, 这对做决定有什么影响? 你的感受又如何的? 
- 对于成千上万的妇女和女孩来说, 发现意外怀孕那一刻就需要做出决定。对于部分人来说做决定 很简单也很直截了当, 但对于另外一部分人来说这是很难、很复杂的;

- 今天我们将讨论有关做流产的决定。我们不会评价流产是对是错, 我们要考虑的是做决定时想到 什么, 弄清楚为什么这些人决定选择流产;

- 当妇女和女孩怀孕以后, 她们有哪些选择? ( 探讨: 生下小孩并抚养孩子; 生下小孩, 让人领 养; 或者终止妊娠 )

2. 将学生分为四或五人一组。给每组一个案例，让他们阅读。填入讲故事的人的名字，然后集体讨论 以下问题 : ( 将问题写在黑板上 )

- 为什么这个女孩会选择做流产;

- 在女孩做决定的过程中, 其他人扮演着什么样的角色; (小组讨论时, 教师在黑板上写上 “妇女 和女孩选择流产的原因” )

3. 全班集合。让第一组阅读他们组的案例，并且给 5-7 分钟讨论以下问题：

- 为什么这个女孩选择做流产;

- 每个人都认为这些是她选择流产的原因吗? 还有其他原因吗? (询问并引导学生探讨与该案例有 关的其他原因 )

- 其他人 ( 性伴侣、家庭、朋友等) 在她做决定的过程中起了什么直接的作用, 或者在她的思想上 起了什么作用;

- 还有其他补充意见吗?

4. 每一组重复以上环节。对每个案例讨论七分钟。

5. 留出10-15分钟回顾以下问题: 
- 你认为黑板上的这些是年轻妇女和女孩选择流产的原因吗? 你还能想到其他的原因吗? (将学生补 充的原因也写在黑板上 )

- 世界范围内, 大多数做过流产的妇女都是已婚的。你能想起一个已婚妇女选择做流产的一些情形 吗?

- 在一些国家, 在很多情况下可以做流产手术, 然而在另一些国家流产则是被禁止的（或者只在极少 数情况下允许流产 );

- 为什么妇女和女孩在法律禁止或不安全的情况下仍然做流产手术呢?

- 当你评判别人的时候, 你并不是在定义别人, 而是在定义自己。

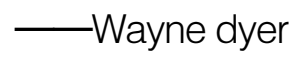

- 你永远不能评判别人的生活, 因为只有每个人知道他或她的痛苦和放弃。感觉自己走对了是一回 事, 但要相信自己走的是唯一的道路是另一回事。

—Paulo Coelho

- 请记住, 我只是个普通人而已。当你要评判我或者决定如何对付我时, 请先站在我的立场思考一 下。这样, 我相信你会多一些理解, 我们可以找到一个中间点, 从此我们可以携手走完之后的路。

-Eric Harvey and Steve Ventura

- 如果可能的话, 我希望让所有人都满意。但在努力实现这个目标的过程中, 我可能会使所有人失 望。因此, 我得出结论: 最佳的办法是对得起自己的良心, 让世界去形成对我们的评判吧, 好也 罢, 不好也罢。

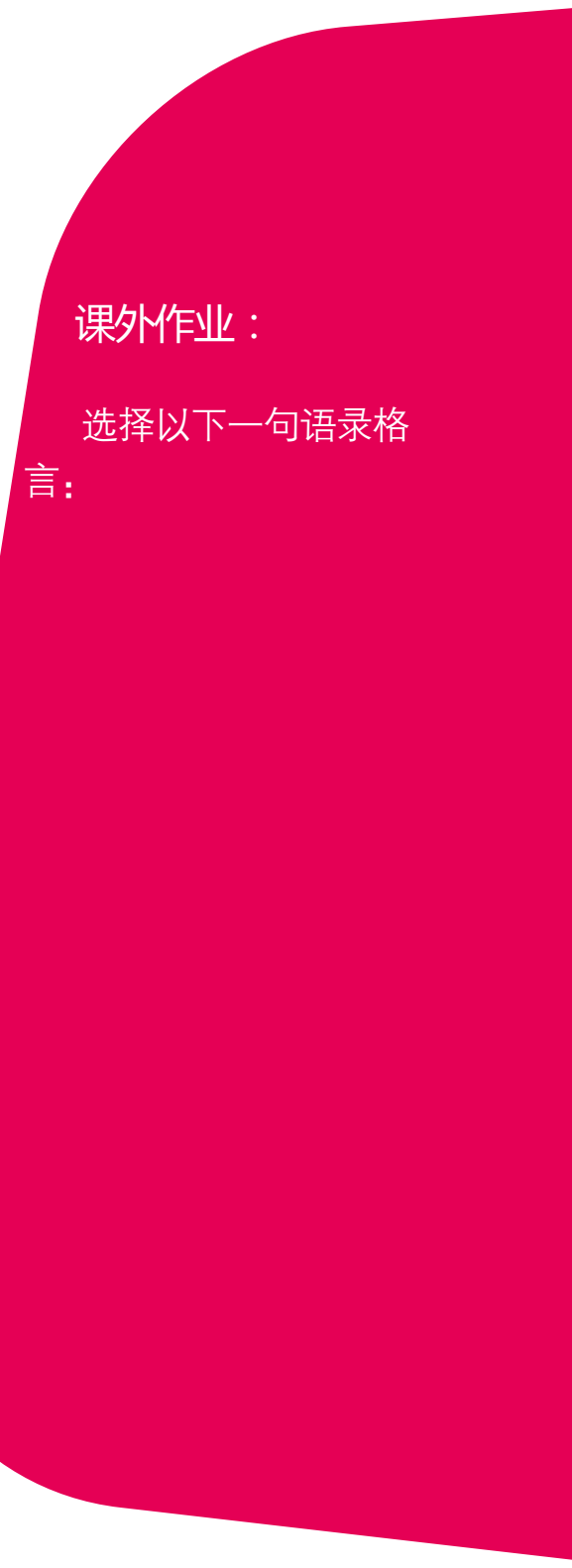


然后, 抄下你选择的格言, 回答以下问题

- 对于这些句子你有什么感想?

- 你同意或不同意作者的观点?

- 这个格言的意思适用于一个女孩在选择终止意外怀孕时的情况吗?

( 学生阅读材料 流产案例 )

第一组材料

我叫 ，我的男朋友路和我都刚满22岁，我们已经交往了两年。我服用避孕药，但是上个月忘记了 几次, 然后, 我发现自己怀孕了。我刚刚找到自己很喜欢但薪水不太高的工作, 我非常享受现在的生 活状态而且不想要小孩。我去一家计生诊所咨询, 护士强烈建议我马上结婚并且把小孩生下来。路也 同意护士的看法, 他认为我早晚要做母亲, 那为什么不现在就做呢? 我感到非常压抑, 因为我觉得有 人在逼我去要一个现在不想要的孩子。我已经决定去另一家诊所, 我听说那里的医生不问太多问题就 可以做流产手术。

\section{第二组材料}

我叫 , 我今年20岁, 是我们村唯一一个在首都上大学的人。我的家庭、朋友和邻居都对我寄予厚 望, 认为我的成功可以改变整个村庄的面貌。在开学后不久, 我开始和一个男生约会, 几个月后我们 开始发生性行为。我们大多数情况下会使用安全套, 但偶尔我们在情急之下就不使用安全套。当我发 现自己怀孕了以后, 我找男朋友商量我们应该怎么办。但他突然开始和我保持距离, 后来我就找不到 
他了。我从一个我们共同的朋友那里听说, 他认为我是在设圈套逼他结婚。我并不想结婚, 但我也不 想变成一个单身母亲, 我想完成我的学业, 有机会实自己的梦想。我本打算向家人求助, 但我害怕他 们会失望, 而我也不想看到他们这样。所以我决定不告诉任何人, 并用自己的生活津贴费去做流产。

第三组材料

我叫—, 我今年17岁。发现女友怀孕以后, 我想: “什么? 茀唷! 不! ”我百感交集, 震惊、害 怕、焦虑、惊异, 甚至还为我有生育能力而感到一点点自豪。但是最终, 我不得不面对这个问题: “我们是抚养孩子, 把孩子送人收养, 还是流产呢? ” 我和女友都还在上学, 我们都知道自己还太年 轻, 当不好父母, 最好的办法还是流产。一个朋友推荐了一家诊所, 我陪女友一起去了那里。诊所的 人解释了大致情况, 在我们离开诊所之前, 她还介绍了一些避孕常识, 并送给我们一盒安全套。我不 得不面对自己的各种情绪, 但我很骄傲我帮助女友度过了难关。

\section{第四组材料}

我叫 , 今年15岁, 和家族的人住在一起。每年, 伯伯、婶子和他们18岁的儿子都会来拜访我们。 今年的某一天, 家里其他人都不在家的时候, 我的堂哥问我, 他可否抚摸我, 还让我也抚摸他。这让 我觉得非常奇怪, 我不太喜欢这样, 但他是比我大的多的堂哥, 我不想让他不高兴。当他开始脱我的 衣服并把我压在身下的时候, 我觉得很害怕, 试着把他推开。但他太强壮了, 他强奸了我。后来发现 自己怀孕以后, 我非常害怕, 我想向妈妈寻求帮助, 但觉得向她解释发生的事情太丢脸了。最后, 我 终于鼓起勇气向妈妈说了一切, 她马上带我去做了流产, 之后便对此事绝口不提。流产后, 我松了一 口气，但我多希望当时能够向朋友倾诉一下自己复杂的心情。 


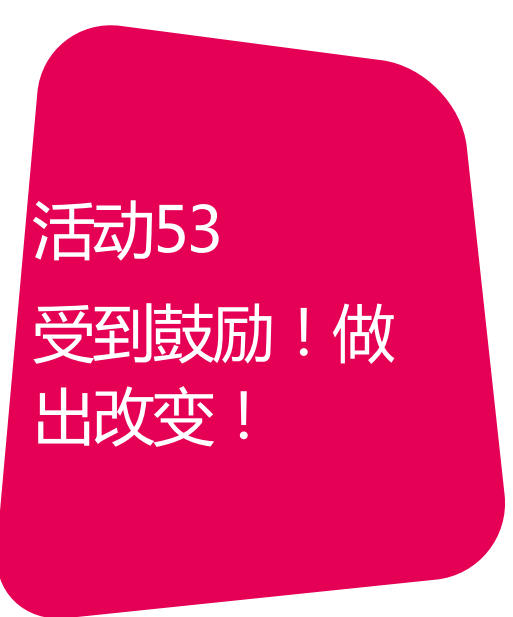

时间：60-90分钟, 取决于你所选择的格言数量

材料 黑板、粉笔; 两页纸的讲义: 有关社会公正和行动主义方面的格言

准备工作：检查格言语录, 删去所有不适合讲解的内容, 你可以添加本国或本地区的素材。考虑到时 间有限, 要控制格言的数量 ( 以及负责朗诵的学生的数量)。将需要引用的格言内容复印 出来或是书写在黑板上

概述: 学生挑选出一句使他们受到激励的名言, 并且探讨如果将名言所传达的信息应用到生活中 将产生什么影响

目的 : 增强学生们改变自己所处世界的动力和信心

\section{步骤}

1. 解释

- 今天我们将探讨为社会公正而奋斗或是改变我们所生活的世界有何意义, 即便达到的改变并不 大。我们会回顾一些人曾经说过的名言, 这些人通过自身的努力给我们的世界带来了相当大的变 化。

- 汶览列表, 挑选出最鼓舞你的格言。然后将其抄录到笔记本中（同时要记下该引言出自何人之 口)。

- 思考你所选择的格言的意义, 将思考的结果写成至少三段话。第一段用来解读该格言在你看来所 具有的含义; 第二段需要解释为什么该格言对于你是有意义的, 以及你选择它的原因; 最后一段 
- 讨论如果你把格言里的内容运用到自己的生活中去将会有什么样的影响（或者你已经将该格言实施 在自己身上, 讨论它对你生活的指导意义)。

( 为学生留出充足的时间书写上述内容。)

2. 询问学生们有谁选择了列表里的第一条格言。请那位学生将他或她思考后的结论读出来 ( 同时要允 许学生选择不去朗读他们希望保密的段落 )。如果不止一个学生选择了同一条格言，可以考虑给予他 们朗读自己见解的选择权。以这种方式，依次进行，直到完成列表上所有格言的阐述。计划安排12分 钟用于格言的分享阅读。

3. 借助以下引导性问题结束这次活动 :

- 从这些格言中你获取哪些主要信息;

- 社会公正活动家从他们投身的事业中获得了哪些正面的个人好处;

- 他们面对的挑战和风险有哪些;

- 对于普通的公民, 就自己关注的社会问题采取行动是否重要?

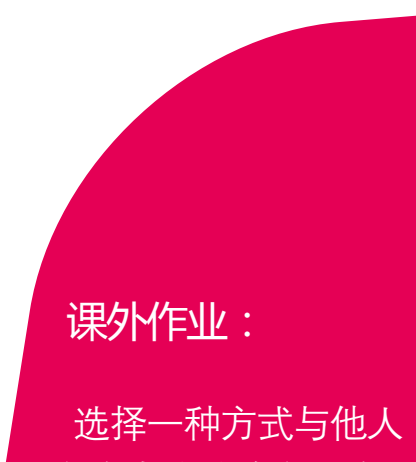
分享你喜欢的格言: 你可 以在家中与家人讨论, 细 心地将它写出下来贴在某 处, 放在你电子邮箱的个 性签名处, 或以其它的方 式与人分享这种励志的 话。写下你的分享过程, 于明日上交。 


\section{( 学生阅读材料 有关社会公正和行动主义方面的格言 )}

你希望看到的世界如何改变，那就从自己开始吧。

一一地, 被印度人尊称为 “国父” 。由他发动的非暴力不合作运动使印度脱离了英国的统治, 而最 终走向独立。他还鼓舞了全世界范围公民权利和自由的运动。

如果你在有权者和无权者的斗争中袖手旁观, 那就意味着你站在了有权者一边, 而决不是所谓的中 立。

一保罗・弗莱雷, 巴西颇具影响力的教育家, 他开启了对当地穷苦大众教育的批判性关注。

如不抗争, 强权从不会主动妥协。以前不曾, 今后也不会有。你会发现人们屈从到什么程度, 强加在 他们身上的不公和冤屈便会到什么程度。而只有当他们以言辞或武力的方式反抗之时, 这种局面才会 终结。暴君的倒行逆施往往是由受欺压者的忍耐程度所决定的。

一一弗里德里克 - 道格拉斯 自幼便沦为奴隶, 但在日后成长为美国反奴隶制运动的领袖。他是作家, 演说家，身为林肯总统的顾问他一直笃信众生平等。

自由不是某个种族垂赐给另一种族的奖赏。他们坚信自由本归自己所有，任谁也无法掠夺。 一一克瓦米 - 恩克鲁玛 加纳的建国者和第一任总统, 同时也是一位影响深远的泛非主义者。 千万不要怀疑一小群有思想、有信念的人能改变世界。这确实是亘古不变的真理。 
一一玛格丽特・米德 美国的人类学家。

这世界是个危险的地方, 不是因为那些做坏事的人, 而是那些袖手旁观的人。

一艾尔伯特 - 爱因斯坦 获得诺贝尔奖的理论物理学家, 以相对论著称于世。曾公开反对纳粹, 他还 是抵制核试验和种族歧视的社会活动家。

当纳粹威胁共产主义者时, 我保持沉默, 因为我不是共产主义者。当他们囚禁社会民主人士时, 我保 持沉默, 因为我不是社会民主人士。当他们进攻工会成员时, 我依旧一言不发, 因为我不是工会成 员。当他们残害犹太人时, 我保持沉默, 因为我不是犹太人。当他们开始对我不利时, 再没有一个人 站出来替我说话。

一马丁 · 尼莫拉 德国著名的神学家和牧师, 曾被送入集中营但幸存了下来。他终其一生都在为反战 奔走呼号。

任何某一地方的不公之事都是对所有正义的威胁。我们受困于一个互相关联的人际网络中而无法逃 脱, 被一件命运的衣衫紧紧捆缚。任何一个单一的事件, 最后都会直接影响到全局。

一马丁 - 路德 - 金 牧师, 美国最著名的人权运动的领袖。非暴力方式的倡导者, 以及为争取各种族 的平等待遇而大声疾呼的活动家。他成为诺贝尔和平奖最年轻的获得者。

普通民众做的就是小事, 但它已经足以改变世界。我的“小事”就是种树。

一万盖瑞 -马塔伊 来自肯尼亚的环保、政治以及社会性别问题的活动家。她发起了 “绿丝带行动” 并于2004年获得诺贝尔和平奖 (她也是第一个荣膺此奖的非裔女性) , 同时还是肯尼亚国会的议员。 


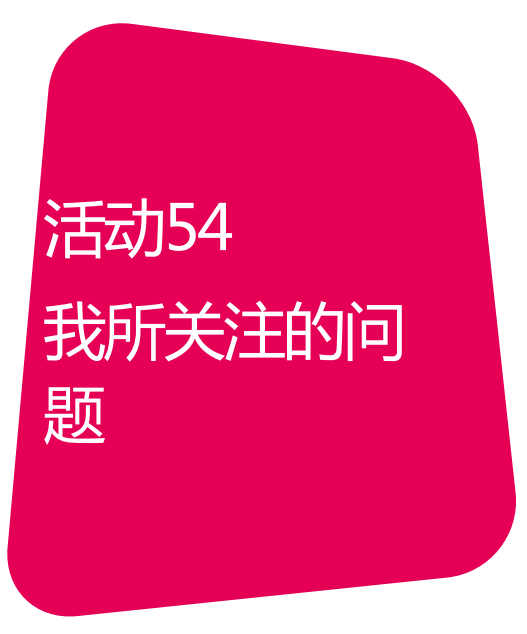

时间：步骤1-4: 45分钟 步骤5-8: 45分钟

材料 黑板、粉笔; 人手一张作业纸（《我所关注的问题》) 和一份讲义(《一封发自肺腑的书 信》) ; 一份《活动指导手册》第八单元的复印件 ( 详见本卷配套书《指导手册》或者登 陆网站: www.popcouncil.org/publications/books/2010_ItsAllOne.asp )。

准备工作: 检查《我所关注的问题》列表, 删除对学生有安全隐患的内容, 增加与当地具体情况有关 的问题。检查讲义《一封发自肺腑的书信》。如果你计划带领学生参与倡导活动项目 (参 考《活动指导手册》第八单元 ), 请保留讲义右栏 “常见措施” 的内容; 如果没有此项计 划, 则删除讲义右栏的内容以防学生在无人引导和支持的情况下自发地采取倡导行动

概述：引导学生发现与他们有关的紧要问题, 并探讨如何在生活中做个积极的先行者

目的: 帮助学生发现并思考他们关心的问题; 锻炼交流和演说技巧; 思考针对问题该如何在生活 中做出积极的改变

1. 介绍主题

- 我们已经了解诸多会影响到我们生活的问题一一尤其是社会性别、健康和权利方面的问题。其中有 哪些特别的问题呢?（探讨我们已经学习过的问题）

- 每位学生要找出一个自己关心的社会问题, 并且这个问题必须与我们先前讨论过的内容有关联。 
2. 给每位学生分发列表《我所关注的问题》。解释 :

- 在纸张的正反面都需填写上自己的姓名; 回顾有关社会公正问题的列表, 向自己发问: 我关心什么 问题?

- 你将看到这份列表内容分成三个类别（社会性别、性健康和性权利）。选择三个你感兴趣的问题。 它们可能属于同一类别, 也有可能来自不同的类别。

- 你也不妨添加一个不包含在列表之中但却是你十分关注的社会公正问题。

3. 安排同性学生以结对的形式参与活动，解释 :

- 每一对学生需用大约20分钟的时间讨论一个或多个在他们看来关系重大的问题。

- 与大家分享你所选择的问题的紧迫之处, 或者需要更深入探讨该问题的理由。你可以讲一个故事与 大家分享;

- 练习专注地相互倾听, 并展开有礼貌的对话;

- 记住: 尊重同学关心的所有问题。平等和尊重从这间教室起步, 从我们对待他人的态度中得到 体现。

4. 集合所有的小组，询问有谁愿意说一说自己挑选了哪个或哪些问题以及挑选的原因。让学生主动 发言。

5. 向学生介绍 “为社会变革而努力” 的概念 ( 倡导)。解释 :

- 有些人投身于大规模的、旨在改变世界的社会运动中。你们有没有听说过人们针对列表上列出的 你们关心的问题而发起的行动;

- 都有哪些行动旨在解决我们正在研究的问题——两性平等、性与生殖健康和权利; (如果需要, 可从《指导手册》第八章或是亲身经历中列举实例 ) 
- 这些行动都给世界带来了一种变化? 试举一例。（你可以援引的实例有：废除缝合妇女和女孩外 阴的陃习、增加在校女学生的数量、男人开始参与反对性暴力的运动、有关流产的法律法规的制 定、增加在校同性恋青年安全感、准许怀孕女孩留校学习 )

6. 介绍 “从小事做起，实现变革”的提议。通过以下要点引发学生的讨论。

- 倡导常常意味着重大的变革。但是即便作出一些很小的努力, 也同样可以在自己和别人的生活中 改进当前的状况;

- 设想并考虑一下你可以采取哪些小的举措, 就能带来变化, 特别是改善性别的不平等或是保护自 己和他人的性与生殖健康和权利（探讨一些观点, 如: 不歧视他人、共同承担通常按性别划分的 责任, 绝不胁迫他人与自己发生性关系、或写信给过去曾遭受过自己粗鲁对待的人表示歎意 )

- 在你的生活中, 有些人作出了一些积极的改变。无论这变化是大还是小, 你能举出这样的例子 吗?

7. 提醒学生在参与倡导时的好处和风险。提问：

- 当一个人参加到社会公正的倡导或活动中时, 这个人可以获得怎样的好处或成长; ( 探讨: 感受 到自己对他人的影响力、建立起新的人际关系、增强了个人能力：如演说和写作能力、受到别人 的钦佩。)

- 当投身社会公正的倡导或活动时, 会面临着哪些风险? ( 探讨: 可能有的社会不赞同、脱离了学 业或是生活中其他的重要事情、没有达成预期目标时的失落、尽管你参与的活动是完全合法和非 暴力的, 但还是有可能被逮捕或面临其他危险。告诉学生安全很重要的。)

8. (可作为课外作业来布置) : 分发讲义，或在黑板上写下讲义里的问题。告诉学生 :

- 就自己关心的问题写一封言辞悬切的信, 并在信中步骤自己为此可以做出的努力; 
- 信可以写给自己、父母、朋友或是亲昵的伴侣, 或是某个社区的负责人。

\section{( 课堂活动材料 我所关注的问题 )}

以下的列表包含了很多人关注并正在努力解决的一些社会问题。列表的内容主要集中于这三个方 面: 社会性别, 性健康以及性权利。 考虑一下哪些问题是你最关心的。也许你关注的问题与这些问题中的某一个非常相似，或者你可能正 思考着一个完全不同的问题。找出三个你关注的问题, 它们可能属于同一类别, 你也有可能思考一个 完全不同的问题。

\section{社会性别问题}

我们的教材强化固有的、刻板的观点;

我们的学校实施某些歧视部分群体的政策;

学校经常发生戏弄和欺侮他人的事情;

在我们的社区男孩承受着被要求表现得坚强或勇敢的压力;

男孩觉得有压力要去加入帮派以证明自己是男人;

女孩缺乏了解 “长大后, 金钱有什么作用” 的机会;

女孩没有足够的机会参与体育活动或者成为体育队的成员; 
女孩没有安全的场所与她们的朋友和同龄人聚会;

广告以对我们有害的方式描绘男性、女性;

许多男青年缺乏去了解如何当一个好父亲的机会;

基于性别的暴力十分常见, 甚至被人所接受（尤其是对妇女和女孩的暴力）;

对待自己的妻子或子女有暴力倾向的男子在饮酒之后变本加厉;

社会性别规范往往会导致进食障碍;

大量的妇女和女孩寻求外科整容手术的帮助以求符合理想的形象;

女孩没有同她们的兄弟一样的上学机会;

不允许怀孕的女孩继续学业;

男孩和女孩在家中得不到平等的待遇;

在社区中人们对社会性别的不公平现象了解有限;

太少的人关注性骚扰问题

其它: 


\section{性健康的问题}

青少年不能获得青年友好型的性与生殖健康机构的服务;

安全套难以获取;

很多我的同龄人对艾滋病病毒一无所知;

在我们所在的地区很多学校都不教授有关艾滋病病毒的知识;

很多人不清楚自己的艾滋病状况;

青年人对自己的身体构造缺乏基本的了解;

性传播疾病在年轻人中间的发病率居高不下;

在世界部分地区很多妇女和女孩遭受着产科瘘管的威胁;

人们不了解或者不够关心产妇死亡率问题;

在很多地区流产依法遭禁, 结果让情况变得危险

其它:

\section{性权利的问题}

在很多地区乱伦现象在很大程度上被忽视;

很多女孩在仍是孩童时就被嫁出;

部分地区仍旧保留着残割妇女和女孩外阴的陋习;

很多女孩遭受 “糖爸爸” 的性剥削; 
很多青年人，尤其是女孩成为性贩卖的受害者;

强奸频繁发生, 甚至被容忍;

人们尚未意识到男孩也正遭受着性侵犯的威胁;

同性恋通常得不到应有的尊重;

很多青年人, 尤其是女孩, 不觉得自己有要求使用安全套的权利;

艾滋病的携带者和艾滋病人得不到足够的帮助和尊重

其它:

务必在这张作业的正反面都填写上自己的名字。 
一封发自肺腑的书信

( 注意: 作积极的改变有着很多种的方式。记住: 从小事开始很重要。另外, 选择安全、合法的行动 也很重要 )

人们常采取的行动有:

决定不歧视他人;

陪同朋友到医疗机构护理;

承诺绝不参与伤害他人的暴力行径;

仔细聆听别人的倾诉;

更多了解关于我所关注的问题的内容;

与我的朋友和家人交谈这些问题;

创立一个社会行动小组;

给一个官员写信;

为我的同龄人撰写一篇文章;

参加一个旨在解决我所关注的社会问题的团体组织;

要求我的学校做出政策调整（与要解决的问题相关）;

在社区策划一次活动。 
敬爱的

我最近正在学习有关性别平等、性健康和性权利的知识。我发现自己能够采取一个小小的但有意 义的行动, 来实现一个积极的改变。我关注的问题是

为实现积极的改变, 我可以做到的是 (从以上的列表上选择一个行动方案, 或者写出另外一个建 议 )

我期待的是

我希望能成为一个

的人.

此致

敬礼! 
附加参考资源

项目案例精选

巴基斯坦的Aahung主要进行培训、调查研究和倡导, 旨在创造一个人人都能享有生殖权利、都能被尊 重、被保护以及得到满足的环境。www.aahung.org

尼日利亚的Conscientizing Male Adolescents主要宗旨在于让青春期男孩认识和了解性别偏见及其行 为, 培养男孩的态度和技能以改变这个现状。如需更多信息请参考www.popcouncil.org/pdfs/qcq/ qcq14.pdf

Dance4Life通过舞蹈家、音乐家、同伴教育者以及携带艾滋病病毒的青年人的参与, 教育并给青年人赋 以权力, 以提高他们与艾滋病病毒和艾滋病抗争的能力。www.dance4life.com

Democracia y Sexualidad(DEMYSEX)是墨西哥的一个组织网络, 其目的在于在民主文化发展过程中加 强性教育、保证性别平等以及性别权利的实践www.demysex.org.mx

尼日利亚的Girls Power Initiative旨在通过为女孩提供充分的信息、技能与行动的机会使其成长为有能力 并自信的女性。www.gpinigeria.org 
美国的Girls Incorporated为女孩提供接受教育的机会, 尤其是那些身处高风险、生活在难以获得相关服 务地区的女孩，使她们有能力去挑战性别歧视，获得成功、独立以及满意的生活。www.girlsinc.org

尼日利亚的“性与生殖健康权利国际中心” (International Centre for Reproductive Health and Sexual Rights(INCRESE)）努力扩大获得性健康、性权利信息及服务的渠道。 www.increse-increse.org

MenEngage是一个全球性的联盟, 主要通过男性和男孩的参与降低性别不平等、促进女性、男性、儿 童的健康状况。www.menengage.org

乌干达的Raising Voices从根本原因诸如传统性别角色以及男女力量的不平衡入手来防止对女性的暴 力。 www.raisingvoices.org

Scenarios USA ( 受西非的Scenarios启发) 发起了一个课程和剧本写作比赛, 内容是反映社会性别的。 获奖的剧本会被拍成短的电影。“什么是真正的阳刚? ” 课程和电影很值得购买, 但这些电影也可以 在网上免费看。www.scenariosusa.org

南非的Sonke Gender Justice Network通过男性、女性、青年人和孩子参与工作来实现性别平等, 防止 基于性别的暴力, 减少艾滋病病毒的传播和艾滋病带来的不良影响。www.genderjustice.org.za 
White Ribbon Campaign旨在教育男性和男孩减少对女性的暴力

www.whiteribbon.ca

\section{课程与活动}

$A B C$ : 中小学人权-实践活动教育手册 ( $A B C$ : Teaching Human Rights-Practical Activities for Primary and Secondary Schools. Office of the United Nations High Commissioner for Human Rights ) 。2003年, 124页。这本手册帮助教育者鼓励人权意识的觉醒和行动。<www.ohchr.org/EN/PublicationsResources/ Pages/TrainingEducation.aspx>同样支持阿拉伯语、中文、法语、俄语和西班牙语。

非洲的变迁: 前进, 主持人培训班指南（African Transformation: The Way Forward, Facilitator's Workshop Guide. Health Communication Partnership, USAID, and Communication for Development Foundation Uganda, ) 2005年, 118页。开展社区性别培训班的工具手册

www.mmc.org/mmc_search.php?sp=\&ref_crmb=Resources\&ref_id=resources\&step=results\&view=deta il\&detail_id=PL_AFR_302\&adv=mat\&swater

Construyendo Derechos: Talleres de Conversaci ó n Para Adolescentes, FLACSO and UNFPA, 2006年 90页。关于性与权利的八个方面做决定的指导。

uww.issuu.com/flacso.chile/docs/construyendo_derechos/15 仅支持西班牙语。

赋权年轻妇女以引领变革: 一个培训手册 (Empowering Young Women to Lead Change: A Traing 
Manual, World YWCA and UNFPA), 2006年124页。适用于那些希望给女青年力量使她们成为领导 并促进她们生活和社区进行积极转变的实施者。www.worldywca.org/world_ywca/communications/ resources/empowering_young_women_to_lead_change. 同样支持西班牙语和法语。

让男性参与社会性别变革(Engaging Boys and Men in Gender Transformation: The Group Education Manual, EngenderHealth and Promundo), 2008年 356页。供人分享的如何让男性参与进来的实践, 探 索性别的社会化和对防止艾滋病病毒的不利影响

www.acquireproject.org/archive/files/7.0_engage_men_as_partners/7.2_resources/7.2.3_tools/Group_ Education_Manual_final.pdf

填平沟壑: 难以开展的教育主题性教育(Filling the Gaps: Hard to Teach Topics in Sexuality Education, Sexuality Information and Education Council of the United States), 1998年 193页。这本手册提供了一些 话题诸如节欲、怀孕、性行为和性别认同的课程安排。

www.siecus.org/pubs/filling_the_gaps.pdf

从性别的角度开展安全的性和艾滋病教育的实践指导手册(Gendering Prevention Practices: A Practical Guide to Working with Gender in Sexual Safety and HIV/AIDS Awareness Education, Nordic Institute for Women's Studies and Gender Research ），2003年52页。这本手册的目的在于促进青年人对于性别的认 知并将其作为促进更安全的性行为的手段。

hivaidsclearinghouse.unesco.org/search/resources/HIV\%20AIDS\%20237.pdf 
性与性别：谁关心它（Gender or Sex: Who Cares?, Ipas and Health and Development Networks ），2001 年 96页。这本培训资源手册旨在提高青少年和青年工作者的技巧以及对于社会性别和生殖健康的理解。 www.ipas.org/Publications/Gender_or_sex_Who_cares.aspx?ht

人权教育系列手册 ( Human Rights Education Series, Human Rights Resource Center, University of Minnesota ），2000年. 这系列的六本书第三次与Amnesty International USA and the Gay, Lesbian and Straight Education Network发行, 主要关注性多元化和权利。

uww1.umn.edu/humanrts/edumat

与性相关的出版物（Internationl Programme on Sexuality Publications, Youth Incentives）. 可从下面网站 下载, 此网站包含了一些小册子、事实列表、课程安排以及倡导简介。

www.youthincentives.org/rutgersnisso_groep/youthincentives/Downloads

同样支持法语。

保持好的改变: 交流性与性别话题的参与式工具包 (Keep the Best Change the Rest: Participatory Tools for Working with Communities on Gender and Sexuality, International HIV/AIDS Alliance )，2007年96页。 这个工具包涵盖了一些帮助社区成员探索性与性别是如何影响他们生活的活动以及一些改善他们人际 关系关系，保护性健康的方法。

www.aidsalliance.org/custom_asp/publications/view.asp?publication_id=257\&language=en 
媒体工具包（MediaLitKit, Framework for k-12 Media literacy, Centre for Media Literacy ），2005. 137页。 这个工具包涵盖了对媒体文化教育的解释, 为媒体文化的应用提供了策略。www.medialit.org

可在www.medialit.org/reading_room/rr4_lessonplan.php 找到课程安排图书馆。

我变化的新身体（The New 'My Changing Body.' Institute for Reproductive Health )。这本教程告诉年轻 人关于青春期和日趋成熟的身体的知识，特别强调了月经和繁殖的知识。www.irh.org

同样支持西班牙语和法语。

和男性讲性、家庭、性暴力和艾滋病 ( One Man Can Workshop Activities: Talking to Men about Gender, Domestic and Sexual Violence and HIV/AIDS, Snoke Gender Justice ），2006. 48页。这本手册提供了 一些活动来鼓励男性和男孩思考他们自己在性别、女性、家庭暴力、性暴力、艾滋病病毒/艾滋病、 民主和人权方面的态度及行为www.genderjustice.org.za/onmancan/complete-one-man-can-toolkit/ download-the-complete-to.html 同样支持南非荷兰语, 法语, 班图语和祖鲁语。

我们的未来: 青少年性与生活技能教育手册 ( Our Future: Sexuality and Life-skills Education for Young People, Grades 4-5, Grades 6-7, and Grades 8-9, International AIDS Alliance ）．2006年和2007年. 132 页, 128页和150页。三册书提供了关于青春期, 友谊, 性别, 性, 怀孕, 性传播疾病, 艾滋病病毒, 艾滋病和吸毒的相关信息及活动, 也提供了家长和监护人如何与孩子谈论性有关话题的一些方法。 www.aidsalliance.org/custom_asp/publications/view.asp?publication_id=211\&language=en 
青年人权运动教育手册 (People's Movement for Human Rights Education (PDHRE-International)) 为推 动人权教育发展而努力。该组织发行了一些关于人权培训的手册和教师材料。

www.pdhre.org

权利和渴望: 性健康教育主持人手册 (Rights and Desire: A Facilitator's Manual to Healthy Sexuality, Breakthrough ），2006年106页。这本手册的主要目的在于提供了一些关于人际关系、性与性别积极的 对话。breakthrough.tv/download/rights-and-desire-a-facilitator-s-manual-to-healthy-sexuality

加强性别平等, 赋于年轻妇女权力的培训手册 ( Sakhi Saheli - Promoting Gender Equity and Empowering Young Women: A Training Manual, CORO for Literacy, Horizons/Population Council, and Instituto Promundo ），2008年136页。这本手册希望促进人们思考应该如何让女青年理解性别规范对她 们生活的影响以及提高她们应对艾滋病病毒和其他生殖健康问题。

www.popcouncil.org/pdfs/horizons/India_SakhiSaheli_Eng.pdf

性与生活技能: 青年性与生殖健康参与式培训活动 ( Sexuality and Life-skills: Participatory Activities on Sexual and Reproductive Health with Young People, International AIDS Alliance ），2008. 172页。为青 年人提供一些活动来帮助他们在成长中了解知识、形成积极的态度、提高技能并享有性与生殖健康。 uww.aidsalliance.org/graphics/secretariat/publications/Sexuality_and_lifeskills.pdf

艾滋病、交流和人际关系技能培训工具包 ( Stepping Stones: A Training Package on HIV/AIDS, 
Communication and Relationship Skills. ACTIONAID )，1995.年240页。这本培训手册主要为培训班提供 了如何探索社会、性和心理需求以及练习在人际关系中应如何表现的培训资料。

www.steppingstonesfeedback.org

这本书可以在www.talcuk.org/books/bs-stepping-stones.htm 买到。

教育资源网站 ( Tools for Change: An Educator's Resource Site, Centre for Research and Education on Violence Against Women and Children at the University of Western Ontario ）. 这个网站为3-9年级的人 (grades 3-9)提供一系列资源以促进健康、平等的人际关系

www.toolsforchange.ca

与男性一起工作 ( Working with Young Men Series, Project H of Instituto Promundo, 以及ECOS-

Comunicacao em Sexualidade, Programa de Apoio ao Pai(PAPAl), Salud Y Genero )，314页。为对 15-24岁的男青年进行性别角色、暴力及性方面的教育提供指导。www.promundo.org.br/396 同样支持 葡萄牙语和西班牙语。

与女性一起工作: 赋权、健康和权利 (Working with Young Women: Empowerment, Health, and Rights, Instituto Promundo, Salud Y Genero, ECOS, Instituto PAPAl and World Education ）.2009.年143pages. 这些手册为15-24岁女青年提供关于性别平等和女权的活动。www.promundo.org.br/352 同样支持葡萄 牙语。 
男性重新定义男性（Yaari Dosti-Young Men Redefine Masculinity, Population Council, CORO for Literacy, MAMTA, and Instituto Promundo ），2006. 110页。这本手册将性别平等作为预防艾滋病病毒传 播的策略。Www.popcouncil.org/pdfs/horizons/yaaridostieng.pdf 同样支持北印度语

uww.popcouncil.org/pdfs/horizons/yaaridostihindi.pdf

年轻男子与艾滋病预防: 行动丛书 ( Young Men and HIV Prevention: A Toolkit for Action, Promundo and UNFPA）．2007年, 两本, 115页和38页。提供概念上的和可实际操作的关于如何设计、应用、评估 艾滋病预防活动的相关信息, 活动可包含性别的角度, 并将女性青年纳入进来。wnw.promundo.org. $\mathrm{br} / 352$ 同样支持葡萄牙语和西班牙语。

\section{教师培训与发展}

防止和应对与学校相关的基于性别暴力的入门手册 ( Doorways: School-Related Gender-Based Violence Prevention and Response, USAID )，2009年. 这三册书（分别提供给教师、学员和社区顾问 ) 支持社区与学校合作, 让学校变得更加安全。

www.usaid.gov/our_work/cross-cutting_programs/wid/doorways.html

性别、艾滋病和人权培训手册 ( Gender, HIV, and Human Rights: A Training Manual, UNIFEM, UNFPA, UNAIDS ）, 2000.年213页。包含了一天和两天的培训, 旨在提高教育者对于艾滋病病毒传播的性别和 人权方面因素的理解。

www.unifem.org/resources/titem_detail.php?ProductionlD=5 
性别或者性: 谁关心它? 为师资培训提供的提示 (Gender or Sex: Who Cares?: Notes for Training of Trainers, Ipas ），2002年68页。这些资源让经验丰富的实施者能够更好地利用Gender or Sex: Who Cares? training这本书（见上面的课程板块）。www.ipas.org/Publications/Gender_or_sex_Notes_for_ Training_of_Trainers.aspx?ht

同样支持克里奥尔语和西班牙语。

人权教育手册: 学习、行动和改变的有效实践 ( The Human Rights Education Handbook: Effective Practices for Learning, Action, and Change, the Human Rights Resource Center, University of Minnesota），2000年这本手册指导教师如何有效地讲解关于人权的知识。

www.umn.edu/humanrts/edumat/hreduseries/hrhandbook/toc.html

我们未来: 为性与生活技能教育而做准备, 教师和社区工作者培训手册 ( Our Future: Preparing to Teach Sexuality and Life Skills, An Awareness Training Manual for Teachers and Community Workers, International AIDS Alliance ), 2008. 年94页。让教师了解如何使用Our Future这本教材 ( 见上面的课程和 活动板块）。

www.aidsalliance.org/custom_asp/pubications/view.asp?publication_id=293

师资培训: 设计和开展有效的人权教育培训手册 ( Training of Trainers: Designing and Delivering Effective Human Rights Education, Training Manual, Equitas-International Centre for Human Rights Education ）,2007年202页。一个六天的基于经验的培训班手册, 主要内容是如何在人权教育方面训 
练培训员。www.equitas.org/english/ed-manuals/ed-manuals.php 同样支持俄语（法语版本即将面 世)。

师资培训手册（Training Trainers for Development, Centre for Development and Population Activities ) , 1995年93页。这本手册提供了一个六天的培训班手册, 主要内容是教授参与式培训的技巧。www. cedpa.org/content/publication/detail/757 同样支持法语和西班牙语。

\section{阅读资料}

体验式学习模式的资料 ( Experiential Learning Cycles: Overview of 9 Experiential Learning Cycle Models )。这个网站为基于经验的学习和应用回顾了9种案例模型。

uww.wilderdom.com/experiential/elc/ExperientialLearningCycle.htm

Gold Dust Resources, Quality Improvement Agency. 这个网站提供了一些资源, 包括提高新的和经验丰 富的老师的技能以及知识水平的各种信息和活动视频。

uww.goldust.org.uk

霍普斯金基金会 ( Hesperian Foundation ). 发行书籍和报纸, 探讨引起较差健康状况的重要社会因素, 为组织提高其社区的健康状况提供了一些建议。www.hesperian.org/publications_download.php 这些资 料总共支持152种语言。 
以学习者为中心的教育方法资料 ( The Learner-centered Teaching Series, Teaching Effectiveness Program, University of Oregon）. 这个网站提供了以学员为中心的教学系列资料, 主要包含四个部分: 概述、课程提纲的演变、教学内容和学员评估。

http://tep.uoregon.edu/worlkshops/teachertraining/learnercentered/learnercentered.html

信息教育网站Paulo Freire and Informal Education, the Encyclopaedia of Informal Education, 2002. 这个 网站提供了对Paolo Freire工作的介绍以及其他的参考材料和链接。

www.infed.org/thinkers/et-freir.htm

培训的变革（Training for Transformation, Volumes1-3 ITDG Publishing ），2002年 462页。50美元。这 三册书的内容是创新的培训技巧，结合了各种参与式教育、组织发展及社区自我依靠的方法与突进。 可从Hesperian Foundation订购: www.hesperian.org/mm5/merchant.mvc?Screen=PROD\&Store_Code=HB\&Product_ Code=B803\&Category_Code=HEB

项目执行工具

加拿大性健康教育指南 ( Canadian Guidelines for Sexual Health Education, Ministry of Health of Canada )，2003. 政府如何开展性教育的一个案例。www.phac-aspc.gc.ca/publicat/cgshe_toc.htm 同 样支持法语。 
CARE. CARE有各种相关的出版物, 包括Addressing the Social Factors That Influence Sexual and Reproductive Health, 以及ISOFI工具包: Tools for Action and Learning on Gender and Sexuality（帮助项 目人员探讨性与性别相关事宜）。uww.care.org/careswork/whatwedo/health/srh/publications.asp 同样 支持法语和西班牙语。

全面性教育指南 ( Developing Guidelines for Comprehensive Sexuality Education, Sexuality Education and Infromation Council of the United States )，1999. 36页。这本手册为教师、政策制定者及社会活动者 提供一步步的指导, 主要内容是指导性教育项目的开展。www.siecus.org/pubs/guidelines/guideintl.pdf 对年轻人性健康的分析(Dynamic Contextual Analysis of Young People's Sexual Health: A Context Specific Approach to Understanding Barriers to, and Opportunities for, Change. Thomas Coram Research Unit, University of Southampton), 2006. 年51页。书中描述了如何进行影响人们性健康的因互进行分析并将其 作为项目的基础。

www.safepassages.soton.ac.uk/pdfs/DCA2.PDF

应对性病、艾滋病的挑战: 基于性别的应对 ( Facing the Challenges of HIV, AIDS, STDs: A Genderbased Response, Royal Tropical Institute, Southern Africa AIDS Information Dissemination Service and World Health Organization, Amsterdam ) , 1995. 56页。为政策制定者和项目实施者提供信息和清单, 包 括将性别与艾滋病、艾滋病病毒及其他性传播疾病的项目结合起来。<data.unaids.org/Topics/Gender/ FacingChallenges_en.pdf>同样支持北印度语。 
艾滋病教育: 性别视角、提示和工具 ( HIV/AIDS Education: A Gender Perspective, Tips and Tools, UNICEF ) ,2002.年24页。为培训师提供正式或不正式场合的背景信息、内容清单以及活动。www. unicef.org/lifeskills/index_14927.html 或www.ibe.unesco.org/AIDS/doc/UNICEF_Gender_Eng.pdf 同样支 持法语和西班牙语。

国际计生联全面性教育框架（IPPF Framework for Comprehensive Sexuality Education. International Planned Parenthood Federation ），2006年9页。提供全面的性教育及可以执行的基本框架。www.ippf. org/en/Resources/Guides-toolkits/Framework+for+Comprehensive+Sexuality+Education.htm 同样支持 法语和西班牙语。

青少年性与生殖健康工作中的主要问题 ( Key Issues in the Implementation of Programmes for Adolescent Sexual and Reproductive Health, Department of Child and Adolescent Health and Development, World Health Organization ），2004. 51页。回顾青少年性与生殖健康项目。 www.who.int/child_adolescent_health/documents/fch_cah_04_3/en/index.html

亚洲青年生殖健康项目RHIYA(The Reproductive Health Initiative for Youth in Asia). 是一个由欧盟和联合 国人口基金首先发起的在南亚和东南亚国家寻求提高10-24岁人口的性与生殖健康的项目。项目提供了 一系列案例研究和项目报告。

www.unfpa.org/eu_partnership/rhiya 
为非政府组织提供的艾滋病、性与生殖健康及权利手册 ( Synergizing HIV/AIDS and Sexual and Reproductive Health and Rights-A Manual for NGOs, AIDSNET ），2006. 22页。为艾滋病预防工作与性 与生殖健康、权利与性别的结合提供证据与指导。

uww.aidsnet.dk/Default.aspx?|D=2366

艾滋病教育工具包 ( Toolkit for Mainstreaming HIV and AIDS in the Education Sector: Guidelines for Development Cooperation Agencies, Joint UN Programme on HIV/ADIS ），2008. 75页。培训材料及资 源。unesdoc.unesco.org/images/0015/001566/156673E.pdf

\author{
培训和技术支援 \\ 非洲区域性行为资源中心 ( ARSRC) , 其总部设在尼日利亚、拉各斯等地区。在非洲地区筹办一些 \\ sexuality institutes。 \\ www.arsrc.org/training/asi/background.html \\ Creating Resources for Empowerment in Action (CREA) 的工作在于赋予女性群体权力使她们能够表达 \\ 自己的需要, 通过技术支援让她们能够更好的实现女性权利, 并在女性权利、性别以及性行为方面开 \\ 展培训。将总部设在印度的CREA在世界各地开展了许多的年度课程计划。 \\ uww.creaworld.org \\ 女孩力量组织Girls Power Initiative, 其总部设在尼日利亚、卡拉巴尔等地区。他们保存了性别发展研究
}


所创建的关于性别、权利、性行为等问题的认识。

www.gpinigeria.org

艾滋病全球青年组织Global Youth Coalition on HIV/AIDS是一个在全球150个国家和地区以青年人为主导 力量的网络, 它具有 4000 个青年领袖和成年人盟友。GYCA培训和授权青年领导人在同龄人中大规模的 开展有关艾滋病与艾滋病病毒的干预措施。

www.youthaidcoalition.org

Instituto Promundo, 其总部设在巴西, 致力于发展Project $\mathrm{H}$ 和Project $\mathrm{M}$, 从事针对青年男性和女性的 性别、权利、性与生殖健康等问题的工作。这个项目提供了技术支援和在性别以及性行为教育方面的 培训。

www.promundo.org.br

人与社会科学国际学校, 阿姆斯特丹大学International School for Humanities and Social Sciences, Universiteit van Amsterdam, 在性行为、文化和社会方面进行研究并且举办暑期学院, 目的是探索不同 文化中性行为的社会层面。为指定国家的与会者提供奖学金。

www.ishss.uva.nl/Summerlnstitute/index.html

National Sexuality Resource Center, San Francisco State University开展有关性行为与文化的暑期学院。

nsrc.sfsu.edu

快乐项目The Pleasure Project, 在牛津、英国和印度都设有办事处。这些办事处对培训者和辅导员提供 培训和技术援助, 让他们能够采取一个积极的工作态度。

www.thepleasureproject.org 
Reprolatina, 其总部设在巴西。主要是提供性与生殖健康、性的多样性和人权方面的培训。

www.reprolatina.org.br/site/html/entrada/index.asp

瑞典性教育协会Swedish Association for Sexuality Education（RFSU）在青年人性与生殖健康、权利、 性的多样性、人权等方面提供技术援助和培训。

uww.rfsu.se/default_en-us.asp

讨论性与生殖健康问题组织Talking about Reproductive and Sexual Health Issues（TARSHI），其总部 设在印度。在南部及东南亚地区提供关于性行为、性别以及权利等方面的培训。

www.tarshi.net

\section{国际性的倡导和政策组织}

加拿大人口与发展行动组织Action Canada for Population and Development ( ACPD) 以促进性与生殖 健康和权利为重点向全球范围发展。

uww.acpd.ca

青年倡导组织Advocates for Youth在美国和全球范围内，提倡发展那些能够使青年人见多识广的政策， 让他们能够对自己在性与生殖健康方面做出的决定负责任。

www.advocatesforyouth.org

国际特赦组织Amnesty International, 是一个人权组织, 它通过安全学校运动来促使女孩得到接受可靠 的教育的机会。在下面的网页可以看到事实与报告工作表: 
www.amnesty.org/en/campaigns/stop-violence-against-women/issues/empowerment-women/safeschools

桥组织BRIDGE, Institute for Development Studies, 为缩小理论、政策和实践之间的差距, 组织在印刷 物与网络上开展性别宣传、访问，并上传多元化的信息。

www.bridge.ids.ac.uk

Catholics for Choice ( CFC ) 致力于推进以正义、保证妇女福祉的承诺为基础的性与生殖健康伦理，人 的道德能力决定了他们的生活。

www.catholicsforchoice.org

健康和性别平等中心Center for Health and Gender Equity (CHANGE) 致力于确保美国在促进性与生殖 健康和权利方面的国际政策和方案。

www.genderhealth.org/index.php

生殖健康权利中心Center for Reproductive Rights是一个世界性的法律宣传组织。它发行了有关青少年 性与生殖权利的出版物:

www.reproductiverights.org/pdf/adolescents\%20bp_FINAL.pdf

www.reproductiverights.org/pdf/BRB_SexEd.pdf

www.reproductiverights.org/pdf/SexualityEducationforAdolescents.pdf

选择:青年与性组织CHOICE for Youth and Sexuality是荷兰的青年组织, 旨在促进青少年性与生殖健康 和权利.

www.choiceforyouth.org 
家庭关爱国际组织Family Care International致力于防止与怀孕和分婏有关的伤害和死亡, 并在国际范围 内确保性与生殖健康和权利。

uww.familycareintl.org/en/home

防止家庭暴力基金会Family Violence Prevention Fund在美国和全世界范围内, 致力于结束在家庭和社会 中, 对妇女和儿童的暴力行为, 并且帮助那些受暴力影响的人。

uww.endabuse.org

Human Rights Watch致力于保护全世界人民的人权, 包括女性的权利; 女同志、同性恋、双性恋、变 性人的权利; 以及有关健康的权利; 例如艾滋病。

wnw.hrw.org

国际同性恋与权利委员会International Gay and Lesbian Human Rights Commission ( IGLHRC ) 不论性 取向或外在表现、性别认同或外在表达亦或是艾滋病毒感染状况, 都要确保全体人民能够充分的享受 人权。

www.iglhrc.org

国际同性恋协会International Lesbian and Gay Association ( ILGA) 是一个全球网络组织, 致力于实现 女同志、同性恋、双性恋、变性人、两性人的平等权利。

uww.ilga.org/index.asp

国际计划生育联合会International Planned Parenthood Federation（IPPF）是一个会员协会的全球网络, 提供性保健和生殖保健的所有权利并为此开展运动。

uww.ippf.org/en 
国际妇女健康组织International Women`s Health Coalition（IVHC）旨在制定健康和人口政策，提供方 案和资金，以促进和保护世界各地的女童和妇女的权利和健康。

wWw.iwhc.org

Ipas全球范围内提高妇女性和生殖权利的能力, 并减少与随胎有关的死亡和伤害。

www.ipas.prg

美国性信息和教育委员会Sexuality Information and Education Council of the United States ( SIECUS ) 为 性教育和性健康权利而工作。

www.siecus.org

全球妇女生殖权利工作网络Women`s Global Network for Reproductive Rights（WGNRR）汇集团体和个 人的力量致力于妇女的生殖权利的倡导。

www.wgnrr.org

世界性健康协会World Association for Sexual Health (WAS) 致力于通过国际机构、区域大陆联合会和 国家组织，以促进性健康的发展。

www.worldsexology.org/index.asp

青年联盟Youth Coalition一个由年轻人组成的国际组织, 致力于促进性健康和生殖权利的年轻人和在国 家、区域和国际各级安全的年轻人有意义的参与和决策。

www.youthcoalition.org 


\section{区域性的宣传和政策组织}

非洲地区性资源中心Africa Regional Sexuality Resource Centre由公司在尼日利亚的健康行动主办, 旨 在对非洲地区通过教育的发展, 实行积极性倾向的方案和政策。增加与知情的公众对话, 对宣传作出 贡献。

www.arsrc.org/index.htm

女性性与生殖健康与权利非洲合作组织Amanitare: African Partnership for the Sexual and Reproductive Health and Rights of Women and Girls致力于保证非洲妇女的身体完整和性健康和生殖权利, 提倡关注 妇女在艾滋病毒、产妇死亡率和避孕方面的权利。

www.amanitare.org.za

亚太地区妇女资源和研究中心Asian-Pacific Resource and Research Centre for Women ( ARROW ) 促 进和保护妇女的健康权利和需求，尤其以性和生殖健康为重点。

www.arrow.org.my

东、中欧性与生殖健康及权利妇女工作网络ASTRA-Central and Eastern European Women`s Network for Sexual and Reproductive Health and Rights一个由非政府组织和个人组成的网络, 倡导性与生殖健 康和权利, 特别是在中欧和东欧地区。

www.astra.org.pl

欧洲青年中心委员会Council of Europe Youth Centre旨在促进欧洲的团结和其公民的尊严, 确保尊重人 权、多元民主和法律的规定。关注 “人人不同，人人平等” 运动:

alldifferent-allequal.info

拉丁美洲与加勒比海地区妇女健康工作网络Latin American and Caribbean Women`s Health Network 
( LACWHN ) 是一个由组织和个人组成的网络，保护健康妇女的人权和公民权。

www.reddesalud.org/englith/sitio/portada.htm

拉美性与人权中心Latin American Center on Sexuality and Human Rights从人权的角度传播一些有关性 的知识, 以帮助消除性别不平等。为减少该地区对性少数群体的歧视的斗争做出贡献。

www.clam.org.br

性资源中心National Sexuality Resource Center，在旧金山州立大学，开发内容、提供信息，在美国， 培训他人对积极的看待性行为以及从社会正义的角度看待性行为。

nsrc.sfsu.edu

加拿大性信息与教育委员会 SIECCAN ( Sex Information and Education Council of Canada ) 促进有关 人类性行为的信息、咨询、研究和出版、公众及专业教育。

www.sieccan.org/index.html

南亚和东南亚性资源中心South and Southeast Asia Resource Centre on Sexuality, 在印度举办了有关生 殖健康和性健康问题讨论, 旨在增加有关性知识、性健康和性在南亚和东南亚的福祉。

www.asiasrc.org

妇女为了妇女权利组织Women for Women`s Human Rights（WWHR），其总部设在土耳其，从事在国 内和国际促进妇女权益。他们的联盟支持在穆斯林社会中的性健康和人身权利。

www.wwhr.org/index.php

穆斯林妇女组织Women Living Under Muslim Laws ( WLUML ) 是一个旨在加强妇女运动、从其生活形 式、条件或由法律和习俗管辖平等和权利的国际声援网络起源于伊斯兰教。 
www.wluml.org/english/index.html

性与生殖健康青年行动网络YouAct, European Youth Network for Sexual and Reproductive Health and Rights一个活跃在欧洲的年轻人中的性和生殖权利领域网络, 旨在使青年们发表自己的看法。

www.youact.org

\section{宣传和政策性文件}

倡导工具Advocacy Kit for Growing Up Global: The Changing Transitions to Adulthood in Developing

Countries, 青年和人口资料局在2005年发出了的倡议这个倡议套件包含案例工作表, 表中总结了从青 年人过渡到成年的专家审查的结果, 还包括宣传提示。

www.advocatesforyouth.org

应对艾滋病的跨国教育Deadly Inertia: A Cross-country Study of Educational Responses to HIV/AIDS, 2005年在布鲁塞尔、比利时发起了关于教育问题的全球运动, 这些出版物审查评论了教育部门应对艾 滋病毒的流行的回应。

wuw.comminit.com

性与性别工具包Gender and Sexuality Cutting Edge Pack, 这个资料包中包含三个文件; 研究性别、性 行为和性权利之间的联系：性别和性的概述报告（51页），支持资源收集（70页），性别和发展（6 页 )。

www.bridge.ids.ac.uk

艾滋病和妇女HIV/AIDS and Women: Resources to Support Policy and Advocacy, 国际妇女论坛中心, 
约40个关于行动的工具、报告和个案研究，以支持对妇女对艾滋病毒和艾滋病的宣传。

www.womenink.org

艾滋病病毒检测: 伴侣间相互的权利和责任HIV testing: The mutual rights and responsibilities of partners, Ruth Dixon-Mueller and Adrienne Germain.2007.Lancet 370(9602):1808-1809. 这篇评论主要 内容为伴侣双方在性关系上权利和责任。

性教育指南: 一个有效的性、人际关系、性病/艾滋病教育方法International Guidelines on Sexuality Education: An evidence informed appoach to effective sex, relationships and HIV/STI education, UNESCO, 2009年6月。这个案例不是一个课程。相反, 它侧重于问 “为什么” 战略关注的问题, 需要 引入或加强性教育和战略关注的问题需要关注 “什么”。它解释性教育是什么? 为什么它很重要。

部长宣言: 教育是为了预防Ministerial Declaration- “Educating To Prevent” 2008年。这一声明是拉丁 美洲和加勒比地区的卫生和教育部长的共同承诺, 提供全面的性教育和性健康和生殖健康服务以加强 艾滋预防工作。它旨在培养全体民众的权益并且反歧视。

性与人际关系教育: 一个社会研究方法Sexuality and relationships education: Toward a social studies approach, Deborah Rogow and Nicole Haberland. 2005.Sex Education 5(4):333-344.本文认为, 社会 研究的框架内进行接地性和关系教育，应该强调性别、社会背景与人权。

女孩, 性暴力与艾滋病Triple Jeopardy: Female Adolescence, Sexual Violence and HIV/AIDS，国际妇女 健康联盟, 2008年。此信息简报提供相关数据, 对性暴力和年轻女孩容易感染艾滋病毒之间的联系作 出解释，并提出政策和方案措施。

Yogyakarta Principles，本文件涉及人权在性倾向和性别认同方面的应用问题。 


\section{青春健康教育指南}

性、性别、艾滋病和人权教育统一行动活动手册

中国计划生育协会

地址: 北京市朝阳区莳药居35号楼 邮编: 100029

电话: 86-10-8465-7984

传真: 86-10-8465-7975

电子邮件: cfpacfpa@chinafpa.org.cn

如需更多信息, 请访问: www.chinafpa.org.cn

www.chinayouthnet.org 